\title{
Une version relative de la conjecture des périodes de Kontsevich-Zagier
}

\author{
Ayoub, Joseph
}

\begin{abstract}
Nous partons d'une série $F=\sum_{r \gg-\infty} f_{r} \cdot \varpi^{r}$ où $\varpi$ est l'indéterminée et les coefficients $f_{r}=f_{r}\left(z_{1}, \ldots, z_{n}\right)$ sont des fonctions holomorphes définies sur un voisinage ouvert du polydisque fermé $\overline{\mathbb{D}}^{n}=\left\{\left(z_{1}, \ldots, z_{n}\right) ;\left|z_{i}\right| \leq 1\right\}$. En intégrant les coefficients de cette série sur le $n$-cube réel $[0,1]^{n}$, on obtient la série de Laurent $\int_{[0,1]^{n}} F$. Lorsque $F$ est algébrique nous dirons que $\int_{[0,1]^{n}} F$ est une série de périodes. Dans cet article, nous cherchons à déterminer les séries algébriques $F$ telles que $\int_{[0,1]^{n}} F$ est nulle. En principle, ceci fournit des informations sur les propriétés de transcendance des séries de périodes. Notre résultat principal rappelle la conjecture des périodes de Kontsevich-Zagier sous une forme remaniée. We start with a series $F=\sum_{r \gg-\infty} f_{r} \cdot \varpi^{r}$ with indeterminate $\varpi$ and where the coefficients $f_{r}=f_{r}\left(z_{1}, \ldots, z_{n}\right)$ are holomorphic functions defined on an open neighborhood of the closed polydisc $\overline{\mathbb{D}}^{n}=\left\{\left(z_{1}, \ldots, z_{n}\right) ;\left|z_{i}\right| \leq 1\right\}$. Integrating the coefficients of this series on the $n$-dimensional real cube $[0,1]^{n}$ yields a Laurent series $\int_{[0,1]^{n}} F$. When $F$ is algebraic we say that $\int_{[0,1]^{n}} F$ is a series of periods. In this article, our goal is to determine the algebraic series $F$ such that $\int_{[0,1]^{n}} F$ is zero. In principle, this gives informations on the transcendence properties of series of periods. Our main result is reminiscent to the Kontsevich-Zagier conjecture on periods in a modified form.
\end{abstract}

DOI: https://doi.org/10.4007/annals.2015.181.3.2

Other titles: A modification of the Kontsevich-Zagier conjecture on periods

Posted at the Zurich Open Repository and Archive, University of Zurich ZORA URL: https://doi.org/10.5167/uzh-111652

Journal Article

Accepted Version

Originally published at:

Ayoub, Joseph (2015). Une version relative de la conjecture des périodes de Kontsevich-Zagier. Annals of Mathematics. Second Series, 181(3):905-992.

DOI: https://doi.org/10.4007/annals.2015.181.3.2 


\title{
UNE VERSION RELATIVE DE LA CONJECTURE DES PÉRIODES DE KONTSEVICH-ZAGIER
}

\author{
par
}

Joseph Ayoub

\begin{abstract}
Résumé. - Nous partons d'une série $F=\sum_{r \gg-\infty} f_{r} \cdot \varpi^{r}$ où $\varpi$ est l'indéterminée et les coefficients $f_{r}=f_{r}\left(z_{1}, \cdots, z_{n}\right)$ sont des fonctions holomorphes définies sur un voisinage ouvert du polydisque fermé $\overline{\mathbb{D}}^{n}=\left\{\left(z_{1}, \cdots, z_{n}\right) ;\left|z_{i}\right| \leq 1\right\}$. En intégrant les coefficients de cette série sur le $n$-cube réel $[0,1]^{n}$, on obtient la série de Laurent $\int_{[0,1]^{n}} F$. Lorsque $F$ est algébrique nous dirons que $\int_{[0,1]^{n}} F$ est une série de périodes. Dans cet article, nous cherchons à déterminer les séries algébriques $F$ telles que $\int_{[0,1]^{n}} F$ est nulle. En principle, ceci fournit des informations sur les propriétés de transcendance des séries de périodes. Notre résultat principal rappelle la conjecture des périodes de Kontsevich-Zagier sous une forme remaniée.

Abstract. - We start with a series $F=\sum_{r \gg-\infty} f_{r} \cdot \varpi^{r}$ with indeterminate $\varpi$ and where the coefficients $f_{r}=f_{r}\left(z_{1}, \cdots, z_{n}\right)$ are holomorphic functions defined on an open neighborhood of the closed polydisc $\overline{\mathbb{D}}^{n}=\left\{\left(z_{1}, \cdots, z_{n}\right) ;\left|z_{i}\right| \leq 1\right\}$. Integrating the coefficients of this series on the $n$-dimensional real cube $[0,1]^{n}$ yields a Laurent series $\int_{[0,1]^{n}} F$. When $F$ is algebraic we say that $\int_{[0,1]^{n}} F$ is a series of periods. In this article, our goal is to determine the algebraic series $F$ such that $\int_{[0,1]^{n}} F$ is zero. In principle, this gives informations on the transcendence properties of series of periods. Our main result is reminiscent to the Kontsevich-Zagier conjecture on periods in a modified form.
\end{abstract}

\section{Table des matières}

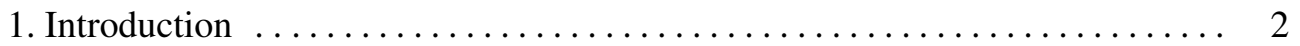

1.1. La conjecture des périodes de Kontsevich-Zagier $\ldots \ldots \ldots \ldots \ldots .2$

1.2. Ce que nous démontrons dans cet article $\ldots \ldots \ldots \ldots \ldots \ldots \ldots . \ldots \ldots$

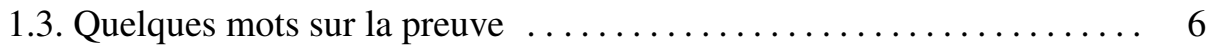

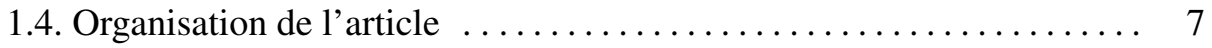

2. Réalisation de Betti tangentielle pour les motifs de Voevodsky ......... 9

2.1. Rappels sur les motifs des schémas $\ldots \ldots \ldots \ldots \ldots \ldots \ldots \ldots \ldots$

2.2. Rappels sur les motifs des variétés rigides $\ldots \ldots \ldots \ldots \ldots \ldots \ldots \ldots$

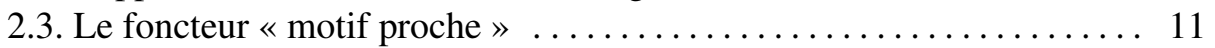

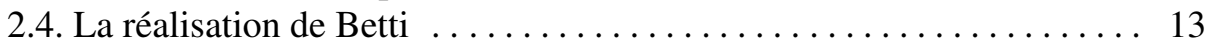

2.5. La réalisation de Betti tangentielle $\ldots \ldots \ldots \ldots \ldots \ldots \ldots \ldots \ldots \ldots$

2.6. Un modèle explicite du foncteur $\mathrm{TgB}^{*}$, première partie $\ldots \ldots \ldots \ldots 17$

2.7. Un modèle explicite du foncteur $\mathrm{TgB}^{*}$, deuxième partie ........ 21

3. Groupes de Galois motiviques et torseurs d'isomorphismes .......... 25

3.1. Les algèbres de Hopf motiviques de $k$ et $F \ldots \ldots \ldots \ldots \ldots \ldots . \ldots \ldots$

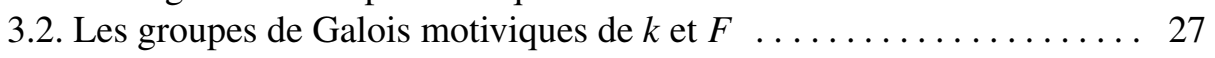

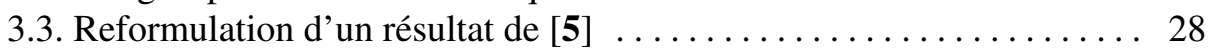

Mots clefs. - Séries de périodes, transcendance, groupe de Galois motivique, géométrie rigide.

L'auteur a bénéficié du soutien partiel du Fond National Suisse de la Recherche Scientifique (NSF), projet no. 200021-124737/1. 
3.4. Application au groupe de Galois motivique relatif de $F / k \ldots \ldots \ldots 29$

3.5. Calculs de torseurs d'isomorphismes, première partie ......... 31

3.6. Calculs de torseurs d'isomorphismes, deuxième partie . . . . . . . 32

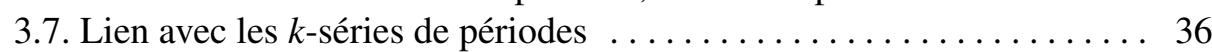

4. Réalisations et évaluations ............................. 37

4.1. Sur la correspondance de Riemann-Hilbert . . . . . . . . . . . . . 37

4.2. Réalisation de Betti et connexions de Gauss-Manin ............ 41

4.3. Démonstration du théorème principal ................. 44

4.4. Le morphisme d'évaluation, première partie $\ldots \ldots \ldots \ldots \ldots \ldots 47$

4.5. Le morphisme d'évaluation, deuxième partie $\ldots \ldots \ldots \ldots \ldots \ldots 50$

4.6. Le morphisme d'évaluation, troisième partie $\ldots \ldots \ldots \ldots \ldots \ldots . \ldots 5$

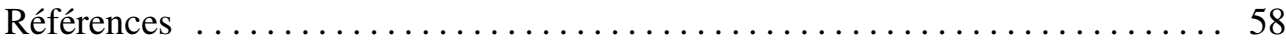

\section{Introduction}

Dans tout l'article, $k$ désignera un corps muni d'un plongement complexe $\sigma: k \hookrightarrow \mathbb{C}$. En général, nous identifions $k$ avec son image dans $\mathbb{C}$.

1.1. La conjecture des périodes de Kontsevich-Zagier. - Commençons par introduire quelques algèbres de fonctions.

Pour $n \in \mathbb{N}$, on notera $\overline{\mathbb{D}}^{n}=\left\{\left(z_{1}, \cdots, z_{n}\right) ;\left|z_{i}\right| \leq 1\right\}$ le polydisque fermé de rayon 1. (En particulier, $\overline{\mathbb{D}}^{0}$ est un singleton.) L'anneau des (germes de) fonctions holomorphes définis sur un voisinage ouvert de $\overline{\mathbb{D}}^{n}$ sera noté comme d'habitude $\mathcal{O}\left(\overline{\mathbb{D}}^{n}\right)$. (En particulier, $\mathcal{O}\left(\overline{\mathbb{D}}^{0}\right)=\mathbb{C}$.) On notera $\mathcal{O}_{k-a l g}\left(\overline{\mathbb{D}}^{n}\right) \subset \mathcal{O}\left(\overline{\mathbb{D}}^{n}\right)$ le sous-anneau des fonctions holomorphes qui sont algébriques sur le corps des fractions rationnelles $k\left(z_{1}, \cdots, z_{n}\right)$. (En particulier, $\mathcal{O}_{k-a l g}\left(\overline{\mathbb{D}}^{0}\right)$ est simplement la clôture algébrique $\bar{k} \subset \mathbb{C}$ de $k$ dans $\mathbb{C}$.) Une fonction dans $\mathcal{O}_{k \text {-alg }}\left(\overline{\mathbb{D}}^{n}\right)$ est dite $k$-algébrique. On identifie $\mathcal{O}\left(\overline{\mathbb{D}}^{n}\right)$ et $\mathcal{O}_{k-a l g}\left(\overline{\mathbb{D}}^{n}\right)$ à des sous-anneaux de $\mathcal{O}\left(\overline{\mathbb{D}}^{n+1}\right)$ et $\mathcal{O}_{k \text {-alg }}\left(\overline{\mathbb{D}}^{n+1}\right)$ en considérant une fonction de $n$ variables comme une fonction de $n+1$ variables constante relativement à la variable $z_{n+1}$. On pose alors $\mathcal{O}\left(\overline{\mathbb{D}}^{\infty}\right)=\bigcup_{n \in \mathbb{N}} \mathcal{O}\left(\overline{\mathbb{D}}^{n}\right)$ et $\mathcal{O}_{k-\text { alg }}\left(\overline{\mathbb{D}}^{\infty}\right)=\bigcup_{n \in \mathbb{N}} \mathcal{O}_{k-\text { alg }}\left(\overline{\mathbb{D}}^{n}\right)$.

On dispose d'un morphisme de $\mathbb{C}$-espaces vectoriels

$$
\int_{[0,1]^{\infty}}: \mathcal{O}\left(\overline{\mathbb{D}}^{\infty}\right) \longrightarrow \mathbb{C}
$$

qui associe à une fonction $f=f\left(z_{1}, \cdots, z_{n}\right)$ son intégrale $\int_{[0,1]^{n}} f$. (Ceci ne dépend pas de $n$.) La restriction de (1) à $\mathcal{O}_{k-a l g}\left(\overline{\mathbb{D}}^{\infty}\right)$ est un morphisme de $k$-espaces vectoriels

$$
\int_{[0,1]^{\infty}}: \mathcal{O}_{k-a l g}\left(\overline{\mathbb{D}}^{\infty}\right) \longrightarrow \mathbb{C} .
$$

L'image de (2) est une sous- $k$-algèbre de $\mathbb{C}$; lorsque $k=\mathbb{Q}$, il s'agit de l'anneau des périodes au sens de [16]. Une formulation de la conjecture des périodes de Kontsevich-Zagier concerne le noyau de (2). On peut l'énoncer comme suit (cf. [4, Rem. 2.109]).

Conjecture 1.1 - (des périodes de Kontsevich-Zagier, forme compacte)

Supposons que k est un corps de nombres. Alors, le noyau de (2) est le sous-k-espace vectoriel engendré par les éléments de la forme

$$
\frac{\partial g}{\partial z_{i}}-\left.g\right|_{z_{i}=1}+\left.g\right|_{z_{i}=0}
$$

avec $g \in \mathcal{O}_{k-a l g}\left(\overline{\mathbb{D}}^{\infty}\right)$ et $i \in \mathbb{N}-\{0\}$.

Dans (3), nous avons noté $\left.g\right|_{z_{i}=\epsilon}$ la substitution dans $g$ de la variable $z_{i}$ par la valeur $\epsilon \in\{0,1\}$. Si $k$ est un corps de nombres, on a $\mathcal{O}_{k-a l g}\left(\overline{\mathbb{D}}^{\infty}\right)=\mathcal{O}_{\mathbb{Q}-a l g}\left(\overline{\mathbb{D}}^{\infty}\right)$ de sorte qu'on ne restreint pas la généralité en prenant $k=\mathbb{Q}$ dans la Conjecture 1.1. 
Remarque 1.2 - Pour comprendre la nature de la Conjecture 1.1 il est utile de remarquer que sa version holomorphe est vraie pour une raison plutôt triviale. En effet, soit $f=f\left(z_{1}, \cdots, z_{n}\right)$ une fonction holomorphe dans le noyau de (1) et raisonnons par récurrence sur $n$. Lorsque $n=0$, il n'y a rien à montrer. Supposons que $n \geq 1$ et soit $g$ une primitive de $f$ par rapport à la variable $z_{n}$, i.e., $\frac{\partial}{\partial z_{n}} g=f$. La fonction $h=f-\left(\frac{\partial}{\partial z_{n}} g-\left.g\right|_{z_{n}=1}+\left.g\right|_{z_{n}=0}\right)$ est aussi dans le noyau de (1) et ne dépend que des variables $z_{1}, \cdots, z_{n-1}$. On peut donc lui appliquer l'hypothèse de récurrence pour l'écrire comme une somme $\sum_{i=1}^{n-1} \frac{\partial}{\partial z_{i}} g_{i}-\left.g_{i}\right|_{z_{i}=1}+\left.g_{i}\right|_{z_{i}=0}$. Ceci permet de conclure.

Bien entendu, le raisonnement ci-dessus ne peut pas fonctionner dans le cas algébrique pour la raison simple mais fondamentale : une primitive d'une fonction algébrique est en général une fonction transcendante. Une autre différence à noter entre le cas algébrique et le cas holomorphe est que la Conjecture 1.1 a peu de chance d'être vraie si l'on fixe le nombre de variables à l'avance. Autrement dit, si $f=f\left(z_{1}, \cdots, z_{n}\right)$ est dans le noyau (2), il est probablement nécessaire d'utiliser des fonctions $g$ qui dépendent de plus de $n+1$ variables si on veut écrire $f$ comme une somme de termes (3).

Dans [16], Kontsevich et Zagier énoncent une conjecture similaire à la Conjecture 1.1 mais beaucoup moins précise; il s'agit de [16, Conj. 1]. Cette conjecture stipule que si une période admet deux présentations intégrales, alors il est possible de passer d'une présentation à l'autre à l'aide des lois élémentaires du calcul intégral, à savoir : linéarité, changement de variable et formule de Stokes. Bien entendu, la restriction fondamentale est que tous les fonctions et domaines d'intégrations auxiliaires sont supposés « algébriques » sur $\mathbb{Q}$. Plus loin dans cet article (cf. [16, $\S 4.1])$, Kontsevich et Zagier donnent une forme plus «scientifique » à leur conjecture. Pour cela, ils introduisent un $\mathbb{Q}$-espace vectoriel, que nous noterons ici $\mathbf{P}_{\mathrm{KZ}}^{e f f}$, ayant pour générateurs des symboles $[(X, Y, n, \omega, \gamma)]$ où $X$ est un $\mathbb{Q}$-schéma de type fini, $Y \subset X$ est un sous-schéma fermé de $X$, $n \in \mathbb{N}$ est un entier naturel, $\omega \in \mathrm{H}_{\mathrm{dR}}^{n}(X, Y)$ est une classe de cohomologie de De Rham algébrique relative et $\gamma \in \mathrm{H}_{n}(X(\mathbb{C}), Y(\mathbb{C})$; $\mathbb{Q})$ est une classe d'homologie singulière relative. Ces générateurs vérifient les relations suivantes :

1. (linéarité) $[(X, Y, n, \omega, \gamma)]$ est linéaire en $\omega$ et $\gamma$.

2. (changement de variable) Si $f:\left(X^{\prime}, Y^{\prime}\right) \longrightarrow(X, Y)$ est un morphisme de paires (défini sur $\mathbb{Q}), \omega \in \mathrm{H}_{\mathrm{dR}}^{n}(X, Y)$ et $\gamma^{\prime} \in \mathrm{H}_{n}\left(X^{\prime}(\mathbb{C}), Y^{\prime}(\mathbb{C}) ; \mathbb{Q}\right)$, alors

$$
\left[\left(X^{\prime}, Y^{\prime}, n, f^{*} \omega, \gamma^{\prime}\right)\right]=\left[\left(X, Y, n, \omega, f_{*} \gamma^{\prime}\right)\right] .
$$

3. (formule de Stokes) $\mathrm{Si} Z \subset Y \subset X$ est une chaîne de sous-schémas fermés, $\beta \in \mathrm{H}_{\mathrm{dR}}^{n-1}(Y, Z)$ et $\gamma \in \mathrm{H}_{n}(X(\mathbb{C}), Y(\mathbb{C}) ; \mathbb{Q})$, alors

$$
[(X, Y, n, \mathrm{~d} \beta, \gamma)]=[(Y, Z, n-1, \beta, \partial \gamma)]
$$

avec

$$
\mathrm{d}: \mathrm{H}_{\mathrm{dR}}^{n-1}(Y, Z) \longrightarrow \mathrm{H}_{\mathrm{dR}}^{n}(X, Y) \quad \text { et } \quad \partial: \mathrm{H}_{n}(X(\mathbb{C}), Y(\mathbb{C}) ; \mathbb{Q}) \longrightarrow \mathrm{H}_{n-1}(Y(\mathbb{C}), Z(\mathbb{C}) ; \mathbb{Q})
$$

les morphismes bord des suites exactes longues usuelles.

On a un morphisme d'évaluation

$$
\mathrm{ev}: \mathbf{P}_{\mathrm{KZ}}^{e f f} \longrightarrow \mathbb{C}
$$

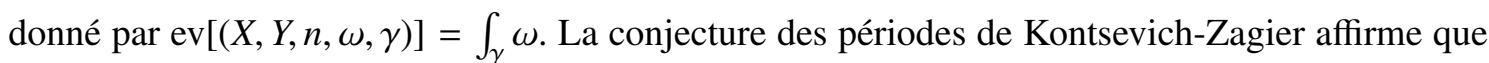
(4) est injectif. Pour expliquer le lien avec la Conjecture 1.1, on note le lemme suivant.

LEMme 1.3 - Il existe un morphisme (de $\mathbb{Q}$-espaces vectoriels $) \mathfrak{f}: \mathcal{O}_{\mathbb{Q}-a l g}\left(\overline{\mathbb{D}}^{\infty}\right) \longrightarrow \mathbf{P}_{\mathrm{KZ}}^{\text {eff }}$ qui rend le triangle

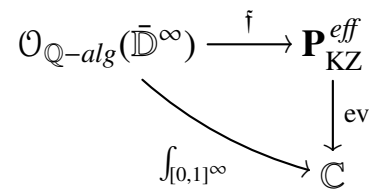

commutatif. 


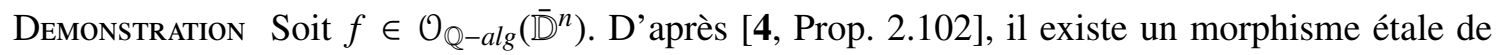
$\mathbb{Q}$-schémas affines $u: U \longrightarrow \mathbb{A}_{\mathbb{Q}}^{n}$ et un triangle commutatif de (pro-)variétés analytiques

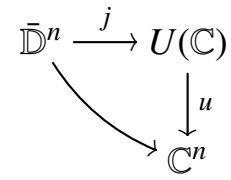

(la flèche oblique étant l'inclusion évidente) tels que $f$ est dans l'image de $j^{*}: \mathcal{O}(U) \longrightarrow \mathcal{O}\left(\overline{\mathbb{D}}^{n}\right)$. On choisit $f^{\prime} \in \mathcal{O}(U)$ tel que $j^{*}\left(f^{\prime}\right)=f$. (Si $U$ est connexe, un tel choix est unique.)

On note $Z \subset U$ l'image inverse par $u$ du diviseur à croisements normaux $\partial \mathbb{A}_{\mathbb{Q}}^{n} \subset \mathbb{A}_{\mathbb{Q}}^{n}$ défini par l'équation $\prod_{\epsilon \in\{0,1\}, 1 \leq i \leq n}\left(z_{i}-\epsilon\right)=0$. On note aussi $\xi \in \mathrm{H}_{n}(U(\mathbb{C}), Z(\mathbb{C}) ; \mathbb{Q})$ la classe d'homologie singulière relative définie dans le complexe des chaînes cubiques par la composition de

$$
[0,1]^{n} \hookrightarrow \overline{\mathbb{D}}^{n} \stackrel{j}{\longrightarrow} U(\mathbb{C}) .
$$

On pose alors

$$
\mathfrak{f}(f)=\left[\left(U, Z, n, f^{\prime} \mathrm{d} z_{1} \wedge \cdots \wedge \mathrm{d} z_{n}, \xi\right)\right] .
$$

En utilisant les relations du type changement de variable dans $\mathbf{P}_{\mathrm{KZ}}^{\text {eff }}$, on vérifie sans peine que le membre de droite dans (5) ne dépend que de $f$. La commutation du triangle de l'énoncé est une conséquence immédiate de la construction.

L'énoncé suivant montre que la Conjecture 1.1 est équivalente à la conjecture des périodes de Kontsevich-Zagier.

FaIt 1.4 - Le morphisme $\mathfrak{f}: \mathcal{O}_{\mathbb{Q}-a l g}\left(\overline{\mathbb{D}}^{\infty}\right) \longrightarrow \mathbf{P}_{\mathrm{KZ}}^{\text {eff }}$ est surjectif et son noyau est le sous- $\mathbb{Q}$-espace vectoriel engendré par les éléments de la forme (3).

Le Fait 1.4 n'a rien d'évident et nous n'en donnerons pas une preuve ici; ceci n'est pas l'objectif de cet article. En effet, il ne servira que pour accréditer l'attribution de la Conjecture 1.1 à Kontsevich et Zagier. Notons toutefois que le Fait 1.4 est une conséquence d'un théorème de comparaison entre le groupe de Galois motivique construit dans [4] et celui de Nori (cf. [18]).

Remarque 1.5 - Le lecteur sceptique s'inquiétera de la « disparition» des relations du type changement de variable dans notre formulation de la conjecture de Kontsevich-Zagier. Nous expliquerons ici comment retrouver ces relations dans le cas d'une seule variable. Soient $f$ et $u$ deux fonctions $k$-algébriques sur $\overline{\mathbb{D}}^{1}$ et supposons que $u(0)=0$ et $u(1)=1$. Considérons le changement de variable $z_{1} \rightsquigarrow u\left(z_{1}\right)$ et montrons que $f\left(z_{1}\right)$ et $u^{\prime}\left(z_{1}\right) f\left(u\left(z_{1}\right)\right)$ définissent la même classe dans le quotient de $\mathcal{O}_{k-a l g}\left(\overline{\mathbb{D}}^{\infty}\right)$ par le sous- $k$-espace vectoriel engendré par les éléments de la forme (3).

En dérivant la fonction $g\left(z_{1}, z_{2}\right)=\left(u\left(z_{1}\right)-z_{1}\right) \cdot f\left(u\left(z_{1}\right) \cdot z_{2}+z_{1} \cdot\left(1-z_{2}\right)\right)$ par rapport à la variable $z_{1}$ et en remarquant que les fonctions $\left.g\right|_{z_{1}=\epsilon}$ sont nulles pour $\epsilon \in\{0,1\}$, on obtient la relation suivante :

$$
\begin{aligned}
\left(u^{\prime}\left(z_{1}\right)-1\right) \cdot & f\left(u\left(z_{1}\right) \cdot z_{2}+z_{1} \cdot\left(1-z_{2}\right)\right) \\
& +\left(u\left(z_{1}\right)-z_{1}\right) \cdot\left(u^{\prime}\left(z_{1}\right) \cdot z_{2}+\left(1-z_{2}\right)\right) \cdot f^{\prime}\left(u\left(z_{1}\right) \cdot z_{2}+z_{1} \cdot\left(1-z_{2}\right)\right) \equiv 0 .
\end{aligned}
$$

En dérivant la fonction $h\left(z_{1}, z_{2}\right)=\left(u^{\prime}\left(z_{1}\right) \cdot z_{2}+\left(1-z_{2}\right)\right) \cdot f\left(u\left(z_{1}\right) \cdot z_{2}+z_{1} \cdot\left(1-z_{2}\right)\right)$ par rapport à la variable $z_{2}$ et en retranchant $\left.h\right|_{z_{2}=1}-\left.h\right|_{z_{2}=0}$, on obtient la relation suivante :

$$
\begin{aligned}
\left(u^{\prime}\left(z_{1}\right)-1\right) \cdot & f\left(u\left(z_{1}\right) \cdot z_{2}+z_{1} \cdot\left(1-z_{2}\right)\right) \\
& +\left(u\left(z_{1}\right)-z_{1}\right) \cdot\left(u^{\prime}\left(z_{1}\right) \cdot z_{2}+\left(1-z_{2}\right)\right) \cdot f^{\prime}\left(u\left(z_{1}\right) \cdot z_{2}+z_{1} \cdot\left(1-z_{2}\right)\right) \\
& \quad-u^{\prime}\left(z_{1}\right) \cdot f\left(u\left(z_{1}\right)\right)+f\left(z_{1}\right) \equiv 0 .
\end{aligned}
$$

Le résultat recherché s'obtient en retranchant les relations (6) et (7). Une méthode similaire montre aussi qu'on a la relation $f\left(z_{1}\right) \equiv f\left(1-z_{1}\right)$. 
1.2. Ce que nous démontrons dans cet article. - Dans toute la suite, $\varpi$ désignera une variable formelle (qui sera plus tard une uniformisante). On pose :

$$
\mathcal{O}^{\dagger}\left(\overline{\mathbb{D}}^{n}\right)=\left(\mathcal{O}\left(\overline{\mathbb{D}}^{n}\right)[[\varpi]]\right)\left[\varpi^{-1}\right] .
$$

Un élément de $\mathcal{O}^{\dagger}\left(\overline{\mathbb{D}}^{n}\right)$ est une série de Laurent $F=\sum_{r \gg-\infty} f_{r} \cdot \varpi^{r}$ où les $f_{r}$ sont des (germes de) fonctions holomorphes sur le polydisque fermé de rayon 1 et de dimension $n$. On pose $\mathcal{O}^{\dagger}\left(\overline{\mathbb{D}}^{\infty}\right)=$ $\bigcup_{n \in \mathbb{N}} \mathcal{O}^{\dagger}\left(\overline{\mathbb{D}}^{n}\right)$.

Une série $F \in \mathcal{O}^{\dagger}\left(\overline{\mathbb{D}}^{n}\right)$ est dite $k$-algébrique si $F$ est algébrique sur le corps des fractions rationnelles $k\left(z_{1}, \cdots, z_{n}, \varpi\right)$. On note $\mathcal{O}_{k \text {-alg }}^{\dagger}\left(\overline{\mathbb{D}}^{n}\right)$ le sous-anneau des séries $k$-algébriques. Si $F=$ $\sum_{r \gg-\infty} f_{r} \cdot \varpi^{r}$ est dans $\mathcal{O}_{k-a l g}^{\dagger}\left(\overline{\mathbb{D}}^{n}\right)$ alors ses coefficients $f_{r}$ sont dans $\mathcal{O}_{k-a l g}\left(\overline{\mathbb{D}}^{n}\right)$. Bien entendu, la réciproque est fausse. On pose $\mathcal{O}_{k-\text { alg }}^{\dagger}\left(\overline{\mathbb{D}}^{\infty}\right)=\bigcup_{n \in \mathbb{N}} \mathcal{O}_{k-\text { alg }}^{\dagger}\left(\overline{\mathbb{D}}^{n}\right)$.

On dispose d'un morphisme de $\mathbb{C}$-espaces vectoriels

$$
\int_{[0,1]^{\infty}}: \mathcal{O}^{\dagger}\left(\overline{\mathbb{D}}^{\infty}\right) \longrightarrow \mathbb{C}((\varpi))
$$

Il associe à $F=\sum_{r \gg-\infty} f_{r} \cdot \varpi^{r} \in \mathcal{O}^{\dagger}\left(\overline{\mathbb{D}}^{\infty}\right)$ la série de Laurent

$$
\int_{[0,1]^{\infty}} F=\sum_{r \gg-\infty}\left(\int_{[0,1]^{\infty}} f_{r}\right) \cdot \varpi^{r}
$$

L'application linéaire (8) identifie le sous-espace vectoriel $\mathcal{O}^{\dagger}\left(\overline{\mathbb{D}}^{0}\right)$ à $\mathbb{C}((\varpi))$. En particulier, elle est surjective.

DéfInItIOn 1.6 - Une série de Laurent dans $\mathbb{C}((\varpi))$ est dite une $k$-série de périodes si elle est dans l'image de l'application k-linéaire

$$
\int_{[0,1]^{\infty}}: \mathcal{O}_{k-a l g}^{\dagger}\left(\overline{\mathbb{D}}^{\infty}\right) \longrightarrow \mathbb{C}((\varpi))
$$

restriction de (8) aux séries $k$-algébriques. Le sous-espace vectoriel des $k$-séries de périodes sera noté $\mathscr{P}^{\dagger}(k)$. C'est une sous- $k(\varpi)$-algèbre de $\mathbb{C}((\varpi))$.

Remarque 1.7 - Comme pour les périodes au sens de Kontsevich et Zagier, certaines « situations géométriques » donnent lieu à des $k$-séries de périodes. Soit $(X, Y, n, \omega, \gamma)$ un quintuplet formé d'un $\mathbb{A}_{k}^{1}=\operatorname{Spec}(k[\varpi])$-schéma de type fini $X$, d'un sous-schéma fermé $Y \subset X$, d'un entier naturel $n \in \mathbb{N}$, d'une classe de cohomologie de De Rham relative $\omega \in \mathrm{H}_{\mathrm{dR}}^{n}\left(X_{\eta}, Y_{\eta}\right)$, avec $X_{\eta}$ et $Y_{\eta}$ les fibres génériques des $\mathbb{A}_{k}^{1}$-schémas $X$ et $Y$, et d'une famille $\gamma=\left(\gamma_{t}\right)_{t \in \mathbb{C}=\mathbb{A}_{k}^{1}(\mathbb{C}), 0<|t|<\epsilon}$ de classes d'homologie singulière $\gamma_{t} \in \mathrm{H}_{n}\left(X_{t}(\mathbb{C}), D_{t}(\mathbb{C}) ; \mathbb{Q}\right)$ avec $\epsilon>0$ un réel suffisamment petit. On suppose que la famille $\gamma=\left(\gamma_{t}\right)_{t}$ est localement constante et invariante par la monodromie autour de $0 \in \mathbb{C}$. (En particulier, elle est déterminée par sa valeur en un point proche de 0.) L'application $t \rightsquigarrow \int_{\gamma_{t}} \omega_{t}$ définit alors un germe de fonctions méromorphes au voisinage de $0 \in \mathbb{C}$ dont le développement en série de Laurent est une $k$-série de périodes. De plus, toutes les $k$-séries de périodes sont obtenues de cette manière. Nous ne démontrerons pas ces assertions ici qui car nous n'en aurons pas besoin.

Les $k$-séries de périodes forment une classe importante de fonctions méromorphes qui rassemble un grand nombre de fonctions classiques. Parmi les exemples on trouve les fonctions polylogarithmes, les inverses des fonctions elliptiques, certaines fonctions hypergéométriques (comme ${ }_{2} F_{1}(a, b ; c ; \varpi)$ avec $a, b, c \in \mathbb{Q}, b>0$ et $c>0$ ), etc. Notre résultat principal concerne le noyau de l'application (9). Pour l'énoncer, nous nous bornerons ici au cas $k=\mathbb{C}$. Pour un énoncé plus général, nous revoyons le lecteur au Théorème 4.25.

ThÉoRème 1.8 - Lorsque $k=\mathbb{C}$, le noyau de (9) est engendré par les éléments de la forme

$$
\frac{\partial G}{\partial z_{i}}-\left.G\right|_{z_{i}=1}+\left.G\right|_{z_{i}=0} \quad \text { et } \quad\left(f-\int_{[0,1]^{\infty}} f\right) \cdot L
$$


avec $G, L \in \mathcal{O}_{\mathbb{C}-a l g}^{\dagger}\left(\overline{\mathbb{D}}^{\infty}\right), i \in \mathbb{N}-\{0\}$ et $f \in \mathcal{O}_{\mathbb{C}-a l g}\left(\overline{\mathbb{D}}^{\infty}\right)$ telle que $f$ et $L$ ne dépendent pas simultanément d'une même variable complexe.

Remarque 1.9 — La ressemblance entre l'énoncé ci-dessus et la Conjecture 1.1 est claire. Il s'agit en fait d'une version relative de cette conjecture. Le Théorème 1.8 affirme qu'une relation linéaire (et même algébrique) entre des séries de périodes n'est jamais le ressort du miracle. Elle s'explique toujours par une raison «presque-algébrique ». En ce sens, il s'agit d'un résultat de transcendance pour des séries de Laurent.

Remarque 1.10 - Il est certainement possible de reformuler le Théorème 1.8 dans l'esprit de $[16, \S 4.1]$ en introduisant une variante convenable de l'espace vectoriel $\mathbf{P}_{\mathrm{KZ}}^{e f f}$ (cf. la Remarque 1.7). Nous ne donnerons pas cet énoncé dont la validité dépendrait d'une variante relative du Fait 1.4.

Remarque 1.11 - Il existe une variante du Théorème 1.8 où les polydisques fermés $\overline{\mathbb{D}}^{n}$ (et $\overline{\mathbb{D}}^{\infty}$ ) sont remplacés par des cubes $\mathbb{I}^{n}$ (et $\mathbb{I}^{\infty}$ ) avec $\mathbb{I}=[0,1] \subset \mathbb{R}$ l'intervalle unité. L'espace vectoriel $\mathcal{O}_{\mathbb{C}-a l g}^{\dagger}\left(\overline{\mathbb{D}}^{\infty}\right)$ est alors remplacé par l'espace vectoriel $\mathcal{O}_{\mathbb{C}-\text { alg }}^{\dagger}\left(\mathbb{I}^{\infty}\right)$ dont les éléments sont les séries de Laurent $F=\sum_{r \gg-\infty} f_{r} \cdot \varpi^{r}$ à coefficients des germes de fonctions holomorphes définies au voisinage de $\mathbb{I}^{n} \subset \mathbb{C}^{n}$ et tels que $F$ est algébrique sur $\mathbb{C}\left(z_{1}, \cdots, z_{n}, \varpi\right)$. Cette variante se démontre en remplaçant partout « $\overline{\mathbb{D}} »$ par $« \mathbb{I} »$ dans la preuve du Théorème 1.8. Hélas, il faudrait aussi faire de même dans [4] et [5]. Une autre possibilité serait d'obtenir cette variante comme une conséquence logique du Théorème 1.8 mais nous ne le ferons pas ici.

1.3. Quelques mots sur la preuve. - Expliquons brièvement l'origine du Théorème 1.8. Elle est motivique : nous obtiendrons le Théorème 1.8 comme une conséquence de la théorie développée dans $[\mathbf{4}, \mathbf{5}]$ et notamment $[\mathbf{5}$, Th. 2.57] qui permet de « borner la taille » du groupe de Galois motivique relatif $\mathbf{G}_{\mid k}^{\text {rel }}\left(k(\varpi), \sigma^{\prime}\right)$ de l'extension transcendante pure $k(\varpi) / k$. (Ce groupe dépend du choix d'une extension $\sigma^{\prime}: k(\varpi) \hookrightarrow \mathbb{C}$ du plongement complexe de $k:$ nous devons donc supposer que l'extension $\mathbb{C} / k$ n'est pas algébrique.) Le résultat [5, Th. 2.57] affirme que l'image d'un certain morphisme de pro-groupes

$$
\pi_{1}\left(\mathcal{V}(\mathbb{C} \backslash \bar{k}), \sigma^{\prime}(\varpi)\right) \longrightarrow \mathbf{G}_{/ k}^{\mathrm{rel}}\left(k(\varpi), \sigma^{\prime}\right)
$$

est Zariski dense. Ci-dessus, $\mathcal{V}(\mathbb{C} \backslash \bar{k})$ est la pro-variété analytique des voisinages Zariski de $\mathbb{C} \backslash \bar{k}$ dans $\mathbb{C}$ naturellement pointée par $\sigma^{\prime}(\varpi)$ et $\pi_{1}\left(\mathcal{V}(\mathbb{C} \backslash \bar{k}), \sigma^{\prime}(\varpi)\right)$ et son pro-groupe fondamental.

Notons $\mathscr{P}_{\text {For }}^{\dagger}(k)$ le quotient du $k$-espace vectoriel $\mathcal{O}_{k-\text { alg }}^{\dagger}\left(\overline{\mathbb{D}}^{\infty}\right)$ par son intersection avec le $\mathbb{C}$ espace vectoriel engendré par les éléments de la forme (10). C'est la version «formelle » de l'anneau des $k$-séries de périodes. On dispose d'un morphisme canonique d'«évaluation »:

$$
\mathscr{P}_{\text {For }}^{\dagger}(k) \longrightarrow \mathbb{C}((\varpi))
$$

d'image $\mathscr{P}^{\dagger}(k)$ et le Théorème 1.8 équivaut à l'injectivité de ce morphisme.

La preuve du Théorème 1.8 comporte deux étapes. La première consiste à établir un lien entre $\mathscr{P}_{\text {For }}^{\dagger}(k)$ et l'algèbre des fonctions régulières sur le torseur des isomorphismes entre un foncteur de réalisation de type Betti associé à un vecteur tangent et un foncteur de réalisation de type De Rham. Plus précisément, nous considérons la réalisation de Betti tangentielle donnée par la composition de

$$
\mathbf{D M}(k(\varpi)) \stackrel{\Psi_{\varpi}}{\longrightarrow} \mathbf{D M}(k) \stackrel{\mathrm{Bti}^{*}}{\longrightarrow} \mathbf{D}(\mathbb{Q})
$$

où $\mathbf{D M}(-)$ est la catégorie des motifs de Voevodsky, $\Psi_{\varpi}$ le foncteur «motif proche » et Bti* la réalisation de Betti. À (13), nous savons associer un groupe de Galois motivique que nous noterons $\mathbf{G}_{\text {mot }}^{\varpi}(k(\varpi), \sigma)$. Par ailleurs, en appliquant (13) à l'objet représentant la cohomologie de De Rham algébrique, on obtient une algèbre de périodes formelles $\mathbf{P}^{\varpi}(k(\varpi), \sigma)$. Le spectre de cette algèbre est le torseur sous le groupe de Galois motivique $\mathbf{G}_{\text {mot }}^{\varpi}(k(\varpi), \sigma)$ qu'il nous faut. En effet, nous 
réaliserons $\mathscr{P}_{\text {For }}^{\dagger}(k)$ comme une sous-algèbre de

$$
\mathbf{P}^{\varpi}(k(\varpi), \sigma) \bigotimes_{\mathbf{P}(k, \sigma), \int} \mathbb{C}
$$

où $\mathbf{P}(k, \sigma)$ est l'anneau des périodes formelles associé au plongement complexe $\sigma: k \hookrightarrow \mathbb{C}$ et $\int: \mathbf{P}(k, \sigma) \longrightarrow \mathbb{C}$ est le morphisme d'évaluation. Un ingrédient essentiel dans cette identification est la théorie des motifs des variétés analytiques rigides [7] et notamment le modèle explicite du foncteur de $\mathbb{B}^{1}$-localisation [7, Th. 2.5.32].

Dans la seconde étape de la preuve, nous considérons les analogues topologiques des constructions précédentes et les morphismes d'évaluation. Ainsi, le groupe pro-algébrique $\mathbf{G}_{\text {mot }}^{\varpi}(k(\varpi), \sigma)$ sera comparé au pro-groupe fondamental de la pro-variété $\mathcal{V}(\mathbb{C} \backslash \bar{k})$ pointée par le vecteur tangent $\frac{\partial}{\partial \varpi}$ en 0 . Plus précisément, on a un morphisme de pro-groupes

$$
\pi_{1}^{\varpi}(\mathcal{V}(\mathbb{C} \backslash \bar{k})) \longrightarrow \mathbf{G}_{\text {mot }}^{\varpi}(k(\varpi), \sigma)
$$

et il résulte de [5, Th. 2.57] que l'adhérence de son image est égale au noyau du morphisme canonique de groupes pro-algébriques

$$
\mathbf{G}_{\mathrm{mot}}^{\varpi}(k(\varpi), \sigma) \longrightarrow \mathbf{G}_{\mathrm{mot}}(k, \sigma) .
$$

Il s'ensuit une propriété similaire pour les torseurs d'isomorphismes bien choisis. En explicitant cette propriété, on trouve un morphisme injectif

$$
\mathbf{P}^{\varpi}(k(\varpi), \sigma) \bigotimes_{\mathbf{P}(k, \sigma), \int} \mathbb{C} \longrightarrow \mathbb{C}((\varpi)) .
$$

En utilisant l'identification obtenue dans la première étape de la preuve, on tombe sur l'énoncé du Théorème 1.8.

Remarque 1.12 — La preuve du Théorème 1.8 repose sur un arsenal de « machineries » plutôt imposant en comparaison avec le caractère élémentaire de son énoncé. Voici une liste non exhaustive de quelques-unes de ces « machineries » qui sont utilisées d'une manière essentielle, bien que parfois cachée.

- La théorie des motifs des variétés algébriques de Voevodsky [20] et notamment l'étude cohomologique des préfaisceaux avec transferts invariants par homotopie.

- Notre théorie des motifs des variétés analytiques rigides [7] et notamment l'étude cohomologique des préfaisceaux avec transferts, surconvergents et invariants par homotopie.

- Notre formalisme des motifs proches, comme développé dans [2], et notamment sa compatibilité avec le formalisme correspondant pour les faisceaux constructibles sur des variétés analytiques [3].

- Le lien entre le formalisme des motifs proches et les motifs des variétés analytiques rigides [7].

- Notre construction des algèbres de Hopf et des groupes de Galois motiviques [4, 5] ainsi que l'étude des groupes de Galois motiviques relatifs et notamment [5, Th. 2.57] qui repose sur le théorème de monodromie de Grothendieck (voir l'appendice de [22]).

- Le calcul de l'algèbre des fonctions régulières sur le torseur d'isomorphismes entre réalisation de Betti et réalisation de De Rham [4, Th. 2.104].

- La correspondance de Riemann-Hilbert sur une courbe et la théorie des équations différentielles à singularités régulières [10].

Ce qui nous amène à la question suivante : existe-il une preuve élémentaire du Théorème 1.8 ? Nous n'en savons rien!

1.4. Organisation de l'article. - Une bonne partie de la Section 2 est consacrée à des rappels sur la théorie des motifs mais on y trouvera aussi des compléments nouveaux et importants. Dans les $\S \S 2.1$ et 2.2 , on rappelle brièvement les constructions des catégories de $S$-motifs (sur un schéma de base $S$ ) et de motifs rigides (sur un corps non-archimédien $K$ ). Dans le $\$ 2.3$ on rappelle 
et on complémente certains résultats fondamentaux de [7]. En particulier, on explique comment la théorie des motifs rigides permet d'obtenir des foncteurs «motif proche». Dans le §2.4, on rappelle la construction de la réalisation de Betti et on utilise cette réalisation pour comparer le foncteur «motif proche » et son analogue topologique. Dans le $\$ 2.5$, on introduit la réalisation de Betti tangentielle qui jouera un rôle important dans la suite. Les $\$ \S 2.6$ et 2.7 constituent le cœur de la Section 2. On y construit un modèle explicite du foncteur de réalisation de Betti tangentielle. Il s'agit là d'un résultat difficile et un ingrédient important de la preuve du Théorème 1.8. L'existence de ce modèle repose sur l'étude fine de la cohomologie des préfaisceaux avec transferts invariants par homotopie dans le contexte algébrique [20] mais aussi dans le contexte analytique rigide [7].

Le but de la Section 3 est d'interpréter l'énoncé du Théorème 1.8 à l'aide de certains torseurs d'isomorphismes sous certains groupes de Galois motiviques. Dans les $\S 3.1$ et 3.2, on introduit les trois groupes de Galois motiviques dont on aura besoin. Le premier, noté $\mathbf{G}_{\text {mot }}(k, \sigma)$, correspond à la réalisation de Betti usuelle. Le second, noté $\mathbf{G}_{\text {mot }}^{\varpi}(F, \sigma)$, correspond à la réalisation de Betti tangentielle. Le troisième, noté $\mathbf{G}_{/ k}^{\text {rel, } \varpi}(F, \sigma)$, mesure la différence entre les deux précédents. (Plus précisément, le second groupe est un produit semi-direct du premier et du troisième.) Dans les $\S \S 3.3$ et 3.4, on utilise [5, Th. 2.49] pour montrer que $\mathbf{G}_{/ k}^{\mathrm{rel}, \varpi}(F, \sigma)$ est un quotient de la complétion pro-algébrique du pro-groupe fondamental de la pro-variété analytique $(\mathbb{C} \backslash \bar{k})=\left\{U^{a n}\right\}_{\emptyset \neq U \subset \mathbb{A}_{k}^{1}}$. Ce résultat est l'un des ingrédients principaux de la preuve du Théorème 1.8. Dans le $\$ 3.5$, on rappelle brièvement (suivant [4]) le calcul de l'algèbre du torseur d'isomorphismes entre la réalisation de Betti et celle de De Rham. Dans le §3.6, on reprend le même calcul pour le torseur d'isomorphismes entre la réalisation de Betti tangentielle et la réalisation de De Rham : mis à part l'utilisation des résultats difficiles des $\$ \$ 2.6$ et 2.7 , la méthode est essentiellement la même. Une fois que les calculs explicites des $\$ \$ 3.5$ et 3.6 sont achevés, on est en mesure de faire le lien avec l'énoncé du Théorème 1.8. C'est l'objet du $\$ 3.7$ (cf. le Théorème 3.27).

Dans la Section 4, on démontre le Théorème 1.8 en se basant sur son interprétation motivique (Théorème 3.27) et en utilisant la reformulation de [5, Th. 2.49]; ces deux ingrédients étaient obtenus dans la Section 3. Dans le §4.1, on rappelle la correspondance de Riemann-Hilbert sur un ouvert Zariski dense $U$ de la droite affine. On utilise cette correspondance pour construire un foncteur fibre $\mathrm{F}_{r h}$ sur la catégorie des systèmes locaux sur $U^{a n}$ à valeurs dans la catégorie des $\mathcal{O}_{U^{-}}$ modules. Ensuite, on calcule explicitement le torseur des isomorphismes entre le foncteur fibre tangentiel en l'origine de la droite affine (qui est à priori en dehors de $U$ ) et le foncteur fibre $\mathrm{F}_{r h}$. Dans le $\$ 4.2$, on étend la connexion de Gauss-Manin aux motifs et on fait le lien avec certains résultats obtenus dans le $\S 4.1$. Dans le $\S 4.3$ on donne la preuve du Théorème 1.8 modulo un résultat technique (à savoir le Théorème 4.21) dont la preuve occupera les $§ \$ 4.4,4.5$ et 4.6.

Remerciements et commentaires. - Je tiens à remercier Simon Pepin Lehalleur pour l'intérêt qu'il a manifesté pour ce travail, pour sa lecture d'une version antérieure de l'article et pour ses commentaires qui ont contribué à améliorer le texte. Je remercie également Yves André d'avoir été le lecteur sceptique de la Remarque 1.5. Enfin, je suis très reconnaissant au rapporteur anonyme pour sa lecture minutieuse et ses suggestions pertinentes qui m'ont permis d'améliorer le texte.

Quelques mois après la rédaction et la soumission du présent article, Annette Huber-Klawitter m'a signalé qu'elle possédait des notes inédites de Madhav Nori où des résultats analogues aux nôtres sont obtenus. Il s'agissait des notes d'un exposé présenté par Madhav Nori à l'université SUNY Buffalo en 2004 et dans lesquelles il démontre qu'une variante du morphisme (14) est injective. (Mis à part que Madhav Nori travaille avec sa propre version des motifs mixtes, il considère aussi un foncteur de réalisation associé à un point complexe générique là où nous utilisons un foncteur de réalisation associé à un point tangentiel.) Je remercie Annette Huber-Klawitter de m'avoir signalé ce travail et Madhav Nori de m'avoir généreusement envoyé ses notes. 


\section{Réalisation de Betti tangentielle pour les motifs de Voevodsky}

Le but de cette section est d'introduire la réalisation de Betti tangentielle (voir la Définition 2.14) et d'établir le Théorème 2.39 qui jouera une rôle essentiel dans la suite. En outre, le lecteur trouvera des rappels succincts et des compléments sur les motifs des variétés algébriques, les motifs des variétés analytiques rigides, les foncteurs «motif proche » et la réalisation de Betti.

On fixe un corps $k$ et un plongement complexe $\sigma: k \hookrightarrow \mathbb{C}$, et on note $\bar{k}$ la clôture algébrique de $k$ dans $\mathbb{C}$. On pose $K=k((\varpi))$. C'est un corps complet non archimédien d'anneau de valuation $K^{\circ}=k[[\varpi]]$, d'idéal maximal $K^{\vee}=\varpi \cdot k[[\varpi]]$ et de corps résiduel $\tilde{K}=k$. On notera aussi $F=k(\varpi)$ qu'on identifiera à un sous-corps dense de $K$.

Dans la suite, on supposera que le corps $k$ est dénombrable. Cette hypothèse technique est souvent superflue mais elle permettra de simplifier l'exposition. D'une part, elle entraîne l'existence d'un plongement complexe $\sigma^{\prime}: F \hookrightarrow \mathbb{C}$ qui prolonge celui de $k$ (ce qui permet de ramener certains énoncés sur la réalisation de Betti tangentielle à des énoncés connus sur la réalisation de Betti usuelle associée à $\sigma^{\prime}$ ). D' autre part, elle servira pour donner un sens à certaines colimites homotopiques dans des catégories triangulées. Ainsi, on pourra prendre des colimites homotopiques indexées par l'ensemble des ouverts non vides de $\mathbb{A}_{k}^{1}$ dans n'importe quelle catégorie triangulée ayant des sommes infinies.

2.1. Rappels sur les motifs des schémas. - Soit $S$ un schéma de base régulier d'égale caractéristique nulle. (Nous serons intéressés par le cas où $S$ est un corps de caractéristique nulle, voire une courbe lisse sur un tel corps.) On note $\mathrm{Sm} / S$ la catégorie des $S$-schémas lisses. La catégorie des correspondances finies (introduite dans [12, Chap. 2]) a pour objets les $S$-schémas lisses et pour morphismes les $S$-correspondances finies; elle est notée $\operatorname{Cor}(S)$. Si $X$ et $Y$ sont dans $\operatorname{Sm} / S$, le groupe des $S$-correspondances finies $\operatorname{Cor}_{S}(X, Y)$ est le $\mathbb{Z}$-module librement engendré par les sous-schémas intègres de $X \times_{S} Y$ qui sont finis et surjectifs sur une composante irréductible de $X$. La définition de la composition dans $\operatorname{Cor}(S)$ utilise les multiplicités d'intersection de Serre.

Dans cet article, nous travaillerons exclusivement à coefficients rationnels. Ainsi, un préfaisceau avec transferts sur $\mathrm{Sm} / S$ est un foncteur additif contravariant de $\operatorname{Cor}(S)$ à valeurs dans la catégorie des $\mathbb{Q}$-espaces vectoriels. Les préfaisceaux avec transferts sur $\mathrm{Sm} / S$ forment une catégorie abélienne de Grothendieck que l'on notera PST $(\mathrm{Sm} / S)$. Si $X \in \mathrm{Sm} / S$, on notera $\mathbb{Q}_{t r}(X)=$ $\operatorname{Cor}_{S}(-, X) \otimes \mathbb{Q}$ le préfaisceau avec transferts représenté par $X$. Rappelons aussi qu'un faisceau avec transferts est un préfaisceau avec transferts dont la restriction à $\mathrm{Sm} / S$ est un faisceau pour la topologie étale. Les faisceaux avec transferts forment une catégorie qu'on notera $\operatorname{Str}(\operatorname{Sm} / S)$. C'est une catégorie abélienne et on dispose d'un foncteur de faisceautisation aét, adjoint à gauche de l'inclusion $\operatorname{Str}(\mathrm{Sm} / S) \hookrightarrow \mathbf{P S T}(\mathrm{Sm} / S)$.

La catégorie $\mathbf{C p l}(\mathbf{P S T}(\mathrm{Sm} / S))$ des complexes de préfaisceaux avec transferts possède une structure de modèles projective ( $\mathbb{A}^{1}$, ét)-locale. Elle est obtenue par une double localisation de Bousfield à partir de la structure projective où les équivalences faibles sont les quasi-isomorphismes de complexes de préfaisceaux et les fibrations sont les épimorphismes. La première localisation de Bousfield fournit la structure projective ét-locale pour laquelle un morphisme de complexes de préfaisceaux avec transferts est une équivalence faible si et seulement si il induit un isomorphisme sur les faisceaux étales associés aux préfaisceaux d'homologie. La catégorie homotopique Hoét $(\mathbf{C p l}(\mathbf{P S T}(\mathrm{Sm} / S)))$ de la structure ét-locale est équivalente à la catégorie dérivée $\mathbf{D}(\operatorname{Str}(\operatorname{Sm} / S))$. La seconde localisation de Bousfield est par rapport à la classe des morphismes $\mathbb{Q}_{t r}\left(\mathbb{A}_{X}^{1}\right)[n] \rightarrow \mathbb{Q}_{t r}(X)[n]$ pour $X \in \mathrm{Sm} / S$ et $n \in \mathbb{Z}$. Elle fournit la structure (A $\mathbb{A}^{1}$, ét)-locale et sa catégorie homotopique est la catégorie triangulée $\mathbf{D M}^{\text {eff }}(S)$ des $S$-motifs effectifs de Voevodsky.

Remarque 2.1 — La définition originale de Voevodsky [12] (voir aussi [24] où le cas relatif est considéré) utilise la topologie de Nisnevich au lieu de la topologie étale. Mais, à coefficients rationnels, un préfaisceau avec transferts est un faisceau Nisnevich si et seulement si il est un faisceau étale. Il s'ensuit que, à coefficients rationnels, les topologies de Nisnevich et étale fournissent les mêmes catégories. 
En utilisant les $T$-spectres, avec $T=\mathbb{Q}_{t r}\left(\mathbb{P}_{S}^{1}, \infty\right)$ le motif de Tate, on peut stabiliser la construction précédente. Ceci fournit la catégorie triangulée $\mathbf{D M}(S)$ des $S$-motifs de Voevodsky où le motif de Tate est inversible pour le produit tensoriel. (Voir par exemple $[\mathbf{9 , 2 1}]$ pour plus de détails.) On dispose d'un foncteur de $T$-suspension infinie

$$
\operatorname{Sus}_{T}^{0}: \mathbf{D M}^{e f f}(S) \longrightarrow \mathbf{D M}(S) .
$$

Lorsque $S$ est le spectre d'un corps, le « cancellation theorem » de Voevodsky [23] affirme que le foncteur (15) est pleinement fidèle. Rien d'analogue n'est connu lorsque la dimension de $S$ est non nulle.

Remarque 2.2 - Le motif de Tate $T[-2] \in \mathbf{D M}^{e f f}(S)$ et sa suspension infinie $\operatorname{Sus}_{T}^{0}(T[-2]) \in$ $\mathbf{D M}(S)$ sont souvent notés $\mathbb{Q}(1)$. Plus généralement, si $M$ est un motif (effectif ou non), les twists de $M$ sont les $M(n)=M \otimes \mathbb{Q}(1)^{\otimes n}$ pour $n \in \mathbb{N}$. Lorsque $M \in \mathbf{D M}(S)$, on peut étendre cette définition pour $n<0$ car $\mathbb{Q}(1)$ est alors inversible pour le produit tensoriel.

Le résultat technique ci-dessous nous sera souvent utile pour ramener des questions sur les catégories motiviques stables à des questions sur les catégories motiviques effectives.

Lemme 2.3 - Soit $\mathbf{E}=\left(\mathbf{E}_{n}, \gamma_{n}\right)_{n \in \mathbb{N}}$ un T-spectre (non symétrique) de complexes de préfaisceaux avec transferts. On a alors un isomorphisme dans $\mathbf{D M}(S)$ :

$$
\mathbf{E} \simeq \operatorname{hocolim}_{n \in \mathbb{N}} \operatorname{Sus}_{T}^{0}\left(\mathbf{E}_{n}\right)(-n)[-2 n]
$$

où les morphismes de transition dans la colimite homotopique sont, à twist et décalage près, les compositions de

$$
\operatorname{Sus}_{T}^{0}\left(\mathbf{E}_{n}\right)(1)[2] \simeq \operatorname{Sus}_{T}^{0}\left(T \otimes \mathbf{E}_{n}\right) \stackrel{\gamma_{n}}{\longrightarrow} \operatorname{Sus}_{T}^{0}\left(\mathbf{E}_{n+1}\right) .
$$

Demonstration C'est standard.

Nous aurons besoin d'une version, en apparence naïve, de la construction précédente, où l'on considère des préfaisceaux sans transferts. (Les détails de cette construction se trouvent dans [2, Chap. 4].) Ainsi, notons $\mathbf{P S h}(\mathrm{Sm} / S$ ) la catégorie des préfaisceaux (en $\mathbb{Q}$-espaces vectoriels) sur $\mathrm{Sm} / S$. Sa catégorie de complexes $\mathbf{C p l}(\mathbf{P S h}(\mathrm{Sm} / S))$ possède également une structure de modèles projective $\left(\mathbb{A}^{1}\right.$, ét)-locale obtenue par une double localisation de Bousfield. La catégorie homotopique de la structure $\left(\mathbb{A}^{1}\right.$, ét)-locale est notée $\mathbf{D} \mathbf{A}^{\text {eff }}$ ét $(S)$. On réservera la notation $\mathbf{D A} \mathbf{A}^{\text {eff }}(S)$ pour la variante Nisnevich de cette construction. En effet, contrairement à ce qui se passe pour les motifs avec transferts, le choix de la topologie importe pour les motifs sans transferts : le foncteur évident $a_{\text {ét }}: \mathbf{D A}^{e f f}(S) \longrightarrow \mathbf{D A}^{e f f}$, ét $(S)$ n'est pas une équivalence de catégories. En utilisant les $T$-spectres, avec $T=\left(\mathbb{P}_{S}^{1}, \infty\right) \otimes \mathbb{Q}$ le motif de Tate, on peut stabiliser la construction précédente. Ceci fournit les catégories triangulées $\mathbf{D A}{ }^{\text {ét }}(S)$ et $\mathbf{D A}(S)$ qui sont liées aux catégories $\mathbf{D} A^{\text {eff, ét }}(S)$ et $\mathbf{D} A^{e f f}(S)$ par des foncteurs de $T$-suspension infinie, notés $\operatorname{Sus}_{T}^{0}$.

Remarquablement, la version naïve n'est pas si naïve. En effet, on dispose d'un foncteur d' « ajout des transferts » $\mathrm{a}_{t r}: \mathbf{P S h}(\mathrm{Sm} / S) \longrightarrow \mathbf{P S T}(\mathrm{Sm} / S)$ qui induit des foncteurs triangulés

$$
\mathrm{La}_{t r}: \mathbf{D A}^{e f f} \text {, ét }(S) \longrightarrow \mathbf{D M}^{e f f}(S) \quad \text { et } \quad \mathrm{La}_{t r}: \mathbf{D A}^{\text {ét }}(S) \longrightarrow \mathbf{D M}(S),
$$

et un théorème de Cisinski-Déglise affirme que les foncteurs (17) sont des équivalences de catégories. Pour une preuve relativement courte, le lecteur peut consulter [4, Ann. B].

2.2. Rappels sur les motifs des variétés rigides. - Les constructions du $§ 2.1$ gardent un sens pour d'autres catégories de « variétés » et en particulier pour les variétés analytiques rigides sur le corps non archimédien $K$. Nous répétons brièvement les constructions dans l'espoir de préciser les notations. (Pour les constructions détaillées, nous renvoyons le lecteur à [7].)

On note $\mathrm{SmRig} / K$ la catégorie des $K$-variétés analytiques rigides lisses. (On supposera que nos variétés analytiques rigides admettent un recouvrement admissible dénombrable par des ouverts affinoïdes et, contrairement à [7], on supposera aussi qu'elles admettent un nombre fini de composantes connexes.) On peut former la catégorie $\operatorname{RigCor}(K)$ ayant les mêmes objets que $\mathrm{SmRig} / K$ et où les morphismes sont donnés par les correspondances finies. Les foncteurs contravariants et 
additifs de $\operatorname{RigCor}(K)$ dans la catégorie des $\mathbb{Q}$-espaces vectoriels sont appelés les préfaisceaux avec transferts sur SmRig/ $K$. Ils forment une catégorie abélienne de Grothendieck qui sera notée PST(SmRig $/ K)$. Si $X \in \operatorname{SmRig} / K$, on note $\mathbb{Q}_{t r}(X)=\operatorname{Cor}_{K}(-, X) \otimes \mathbb{Q}$ le préfaisceau avec transferts représenté par $X$. Un faisceau avec transferts est un préfaisceau avec transferts dont la restriction à $\mathrm{SmRig} / K$ est un faisceau pour la topologie étale. (Vu qu'on travaille à coefficients rationnels, ceci équivaut à demander que cette restriction est un faisceau pour la topologie de Nisnevich.) Les faisceaux avec transferts forment une catégorie abélienne qui sera notée $\operatorname{Str}(\operatorname{SmRig} / K)$.

La catégorie $\mathbf{C p l}(\mathbf{P S T}(\operatorname{SmRig} / K))$ des complexes de préfaisceaux avec transferts possède une structure de modèles projective ( $\mathbb{B}^{1}$, ét)-locale obtenue par une double localisation de Bousfield. La première localisation est topologique et fournit une catégorie homotopique équivalente à la catégorie dérivée $\mathbf{D}(\operatorname{Str}(\operatorname{SmRig} / K))$. La seconde localisation est par rapport à la classe des morphismes $\mathbb{Q}_{t r}\left(\mathbb{B}_{X}^{1}\right)[n] \longrightarrow \mathbb{Q}_{t r}(X)[n]$ avec $X \in \operatorname{SmRig} / K$ et $n \in \mathbb{Z}$. (Bien entendu, $\mathbb{B}_{K}^{1}=\operatorname{Spm}(K\{t\}$ ) est la boule unité de Tate.) La catégorie homotopique de la structure ( $\mathbb{B}^{1}$, ét)-locale est la catégorie triangulée $\mathbf{R i g D M}^{\text {eff }}(K)$ des motifs (effectifs) des variétés analytiques rigides.

Notons $\mathbb{P}_{K}^{\text {, anr }}$ la variété analytique rigide associée à la doite projective sur $K$. En utilisant les $T$-spectres, avec $T=\mathbb{Q}_{t r}\left(\mathbb{P}_{K}^{1, a n r}, \infty\right)$ le motif de Tate, on obtient la catégorie $\operatorname{RigDM}(K)$ où le motif de Tate est inversible pour le produit tensoriel. On a aussi un foncteur de $T$-suspension infinie

$$
\operatorname{Sus}_{T}^{0}: \operatorname{RigDM}^{e f f}(K) \longrightarrow \operatorname{RigDM}(K)
$$

qui est pleinement fidèle par l'analogue analytique rigide du « cancellation theorem » de Voevodsky (cf. [7, Cor. 2.5.49]). Les twists de Tate dans les catégories $\operatorname{RigDM}^{\text {eff }}(K)$ et $\operatorname{RigDM}(K)$ sont définis de la même manière que dans le cas algébrique (cf. la Remarque 2.2). L'analogue rigide du Lemme 2.3 est aussi vrai.

On dispose également de la variante sans transferts de la construction précédente. Ici, on considère la catégorie $\mathbf{P S h}(\mathrm{SmRig} / K$ ) des préfaisceaux (en $\mathbb{Q}$-espaces vectoriels) sur $\mathrm{SmRig} / K$. Sa catégorie de complexes $\mathbf{C p l}(\mathbf{P S h}(\operatorname{SmRig} / K))$ possède une structure de modèles projective ( $\mathbb{B}^{1}$, ét)locale obtenue par double localisation de Bousfield. La catégorie homotopique associée est notée $\operatorname{RigDA}^{e f f}$, ét $(K)$. On réservera la notation $\operatorname{RigDA}^{e f f}(K)$ pour la variante Nisnevich de cette construction. Comme pour les schémas, cette distinction importe lorsqu'on n'utilise pas les transferts. En utilisant les $T$-spectres, avec $T=\left(\mathbb{P}_{K}^{1, \text { anr }}, \infty\right) \otimes \mathbb{Q}$ le motif de Tate, on peut stabiliser la construction précédente. Ceci fournit les catégories triangulées $\operatorname{RigDA}^{\mathrm{e}}{ }^{\mathrm{t}}(K)$ et $\operatorname{RigDA}(K)$ qui sont liées aux catégories $\operatorname{RigDA}^{e f f}$, ét $(K)$ et $\operatorname{RigDA}^{e f f}(K)$ par des foncteurs de $T$-suspension infinie, notés $\operatorname{Sus}_{T}^{0}$.

On dispose d'un foncteur d' " ajout des transferts » $\mathrm{a}_{t r}: \mathbf{P S h}(\mathrm{SmRig} / K) \longrightarrow \mathbf{P S T}(\mathrm{SmRig} / K)$. Il induit des foncteurs triangulés

$$
\mathrm{La}_{t r}: \operatorname{RigDA}^{e f f} \text {,ét }(K) \longrightarrow \operatorname{RigDM}^{e f f}(K) \quad \text { et } \quad \operatorname{La}_{t r}: \operatorname{RigDA}^{\text {ét }}(K) \longrightarrow \operatorname{RigDM}(K)
$$

Comme pour les schémas, les foncteurs (19) sont des équivalences de catégories. (Nous ne disposons pas de référence pour cela, mais le lecteur vérifiera sans peine que la preuve présentée dans $[4$, Ann. B] s'étend littéralement au cas des variétés analytiques rigides.)

2.3. Le foncteur « motif proche». - Étant donné un $F$-schéma $X$, notons $X^{\text {anr }}$ la variété analytique rigide associée. Le foncteur $(-)^{a n r}$ induit un foncteur triangulé

$$
\operatorname{Rig}^{*}: \mathbf{D M}^{e f f}(F) \longrightarrow \operatorname{RigDM}^{e f f}(K)
$$

qui envoie $\mathbb{Q}_{t r}(X) \operatorname{sur} \mathbb{Q}_{t r}\left(X^{a n r}\right)$. Bien entendu, nous avons aussi la variante stable et les variantes sans transferts de (20); elles seront également désignées par Rig*

Étant donnés $r \in \mathbb{N}-\{0\}$ et $X \in \mathrm{Sm} / k$, notons $Q_{r}^{g m}(X)$ le $\mathbf{G}_{\boldsymbol{m} k}$-schéma $X \times_{k} \mathbf{G}_{\boldsymbol{m} k}$ ayant pour morphisme structural la composition de

$$
X \times{ }_{k} \mathbf{G}_{\boldsymbol{m} k} \longrightarrow \mathbf{G}_{\boldsymbol{m} k} \stackrel{(-)^{r}}{\longrightarrow} \mathbf{G}_{\boldsymbol{m} k} .
$$


Notons quDM ${ }^{e f f}(k)$ la plus petite sous-catégorie triangulée de $\mathbf{D M}^{e f f}\left(\mathbf{G}_{\boldsymbol{m}_{k}}\right)$, stable par sommes infinies et contenant les objets de la forme $\mathbb{Q}_{t r}\left(Q_{r}^{g m}(X)\right)$ avec $r \in \mathbb{N}-\{0\}$ et $X$ un $k$-schéma lisse. Un des résultats fondamentaux de [7, Chap. 2] s'énonce de la manière suivante.

THÉORÈme 2.4 - Le foncteur composé

$$
\mathfrak{r}: \mathbf{q u D M}^{\text {eff }}(k) \hookrightarrow \mathbf{D M}^{\text {eff }}\left(\mathbf{G}_{\boldsymbol{m} k}\right) \stackrel{\varpi^{*}}{\longrightarrow} \mathbf{D M}^{\text {eff }}(F) \stackrel{\operatorname{Rig}^{*}}{\longrightarrow} \mathbf{R i g D M}^{\text {eff }}(K)
$$

est une équivalence de catégories. (Ci-dessus, $\varpi^{*}$ est le foncteur "image inverse » suivant le $F$-point $\varpi \in \mathbf{G}_{\boldsymbol{m}_{k}}(F)$.)

À vrai dire, l'énoncé ci-dessus est une version effective de [7, Th. 2.5.51] et ne figure pas tel quel dans [7]. Expliquons donc comment l'obtenir. Notons quDM( $k$ ) la plus petite souscatégorie triangulée de $\mathbf{D M}\left(\mathbf{G}_{\boldsymbol{m} k}\right)$, stable par sommes infinies et contenant les objets de la forme $\operatorname{Sus}_{T}^{0}\left(\mathbb{Q}_{t r}\left(Q_{r}^{g m}(X)\right)\right)(n)$ avec $r \in \mathbb{N}-\{0\}, n \in \mathbb{Z}$ et $X$ un $k$-schéma lisse. C'est la version stable de $\mathbf{q u D M}^{\text {eff }}(k)$. Le foncteur de $T$-suspension infinie induit un foncteur

$$
\operatorname{Sus}_{T}^{0}: \mathbf{q u D M}^{e f f}(k) \longrightarrow \mathbf{q u D M}(k) \text {. }
$$

Nous aurons besoin du résultat suivant.

LEMME 2.5 - Le foncteur (22) est pleinement fidèle.

Demonstration Le «cancellation theorem » de Voevodsky n'est pas disponible pour les motifs au-dessus de $\mathbf{G}_{\boldsymbol{m} k}$. Heureusement, il est possible de descendre le problème à $\operatorname{Spec}(k)$.

Soient $X$ et $Y$ des $k$-schémas lisses, et $r$ et $s$ des entiers non nuls. D'après [7, Lem. 1.3.32], il suffira de montrer que les homomorphismes

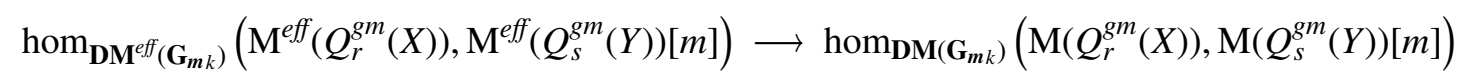

sont des bijections pour tout $m \in \mathbb{Z}$. (Ci-dessus, nous avons écrit $\mathbf{M}^{\text {eff }}(-)$ et $\mathrm{M}(-)$ au lieu de $\mathbb{Q}_{t r}(-)$ et $\operatorname{Sus}_{T}^{0}\left(\mathbb{Q}_{t r}(-)\right)$.) Or, il est facile de voir que $\mathbb{Q}_{t r}\left(Q_{r}^{g m}(X)\right)$ est un facteur direct de $\mathbb{Q}_{t r}\left(Q_{s \cdot r}^{g m}(X)\right)$ et de même avec $Y$ à la place de $X$. On peut donc supposer que $r=s$.

En notant $e_{r}: \mathbf{G}_{\boldsymbol{m} k} \longrightarrow \mathbf{G}_{\boldsymbol{m} k}$ le morphisme d'élévation à la puissance $r$, on a

$$
\mathrm{M}^{e f f}\left(Q_{r}^{g m}(X)\right)=e_{r \sharp} e_{r}^{*} \mathrm{M}^{e f f}\left(Q_{1}^{g m}(X)\right)
$$

et de même pour $\mathrm{M}\left(Q_{r}^{g m}(X)\right)$. Par ailleurs, on a $e_{r}^{*} \mathrm{M}^{e f f}\left(Q_{r}^{g m}(Y)\right)=\mathrm{M}^{e f f}\left(Q_{1}^{g m}\left(Y \times_{k}\left(\mu_{r}\right)_{k}\right)\right)$ et de même pour $\mathrm{M}\left(Q_{r}^{g m}(Y)\right)$. Ainsi, vu l'adjonction $\left(e_{r \sharp}, e_{r}^{*}\right)$ et quitte à remplacer $Y$ par $Y \times_{k}\left(\mu_{r}\right)_{k}$, nous sommes ramenés à montrer que les homomorphismes

$$
\operatorname{hom}_{\mathbf{D M}} \mathbf{M}_{\left(\mathbf{G}_{m k}\right)}\left(\mathbf{M}^{e f f}\left(Q_{1}^{g m}(X)\right), \mathbf{M}^{e f f}\left(Q_{1}^{g m}(Y)\right)[m]\right) \longrightarrow \operatorname{hom}_{\mathbf{D M}\left(\mathbf{G}_{m k}\right)}\left(\mathbf{M}\left(Q_{1}^{g m}(X)\right), \mathbf{M}\left(Q_{1}^{g m}(Y)\right)[m]\right)
$$

sont des bijections pour tout $m \in \mathbb{Z}$.

Puisque $Q_{1}^{g m}(Y)=Y \times_{k} \mathbf{G}_{m k}$, on a $\mathrm{M}^{e f f}\left(Q_{1}^{g m}(Y)\right)=p^{*} \mathbf{M}^{e f f}(Y)$ et $\mathrm{M}\left(Q_{1}^{g m}(Y)\right)=p^{*} \mathrm{M}(Y)$ avec $p: \mathbf{G}_{\boldsymbol{m} k} \longrightarrow \operatorname{Spec}(k)$ la projection structurale. Vu l'adjonction $\left(p_{\sharp}, p^{*}\right)$, nous sommes ramenés à montrer que les homomorphismes

$$
\operatorname{hom}_{\mathbf{D M}^{e f f}(k)}\left(\mathbf{M}^{e f f}\left(X \times_{k} \mathbf{G}_{\boldsymbol{m} k}\right), \mathrm{M}^{e f f}(Y)[m]\right) \longrightarrow \operatorname{hom}_{\mathbf{D M}(k)}\left(\mathrm{M}\left(X \times_{k} \mathbf{G}_{\boldsymbol{m} k}\right), \mathrm{M}(Y)[m]\right)
$$

sont des bijections pour tout $m \in \mathbb{Z}$. Le « cancellation theorem» de Voevodsky [23] permet maintenant de conclure.

DÉMOnSTRATION DU ThÉoRÈMe 2.4 La catégorie quDM $(k)$ est égale à la catégorie QUDM $(k)$ introduite dans [7, Déf. 2.5.50]. (Ceci découle du fait que $\mathbb{Q}_{t r}\left(Q_{r}^{g m}(X, f)\right)$ est un facteur direct de $\mathbb{Q}_{t r}\left(Q_{r}^{g m}\left(X\left[f^{1 / r}\right]\right)\right)$.) En utilisant le Lemme 2.5 et le « cancellation theorem » pour les motifs des variétés analytiques rigides (cf. [7, Cor. 2.5.49]), on déduit de [7, Th. 2.5.51] que la composition de (21) est un foncteur pleinement fidèle. Il reste donc à voir que l'image de la composition de (21) contient un système de générateurs de la catégorie triangulée avec sommes infinies $\operatorname{RigDM}^{\text {eff }}(K)$.

D'après [7, Th. 1.2.36], un tel système de générateurs est donné par les objets de la forme $\mathbb{Q}_{t r}\left(Q_{r}^{r i g}(X)\right)$ avec $r \in \mathbb{N}-\{0\}, X \in \mathrm{Sm} / k$ et $Q_{r}^{r i g}(X)$ la fibre générique du schéma formel $X\left[\left[\varpi^{1 / r}\right]\right]=$ $\left(X \otimes_{k} K^{\circ}\left[\varpi^{1 / r}\right]\right) /(\varpi)$. (On utilise le fait que $\mathbb{Q}_{t r}\left(Q_{r}^{r i g}(X, f)\right)$ est un facteur direct de $\mathbb{Q}_{t r}\left(Q_{r}^{r i g}\left(X\left[f^{1 / r}\right]\right)\right)$ 
pour simplifier le système de générateurs fourni par [7, Th. 1.2.36].) D'après [7, Th. 1.3.11], le morphisme canonique $\mathbb{Q}_{t r}\left(Q_{r}^{r i g}(X)\right) \longrightarrow \mathbb{Q}_{t r}\left(\left(X \otimes_{k} F\right)^{a n r}\right)$ est une équivalence ( $\mathbb{B}^{1}$, ét)-locale. Le résultat recherché s'ensuit puisque $\mathbb{Q}_{t r}\left(\left(X \otimes_{k} F\right)^{a n r}\right)$ est l'image de $\mathbb{Q}_{t r}\left(Q_{r}^{g m}(X)\right)$ par la composition de (21).

On fixe une fois pour toute une équivalence de catégories $\operatorname{RigDM}^{e f f}(K) \simeq \mathbf{q u D M}^{\text {eff }}(k)$ qui est quasi-inverse à l'équivalence $\mathfrak{F}$. La définition suivante est motivée par [7, Scholie 1.3.26, (2)].

DéfinItion 2.6 - Le foncteur « motif proche » $\Psi_{\varpi}: \mathbf{D M}^{\text {eff }}(F) \longrightarrow \mathbf{D M}^{\text {eff }}(k)$ est la composition de

$$
\mathbf{D M}^{e f f}(F) \stackrel{\operatorname{Rig}^{*}}{\longrightarrow} \operatorname{RigDM}^{e f f}(K) \simeq \mathbf{q u D M}^{e f f}(k) \hookrightarrow \mathbf{D M}^{\text {eff }}\left(\mathbf{G}_{\boldsymbol{m}}\right) \stackrel{1^{*}}{\longrightarrow} \mathbf{D M}^{e f f}(k) .
$$

(Ci-dessus, $1^{*}$ est le foncteur «image inverse » suivant la section unité $1 \in \mathbf{G}_{\boldsymbol{m} k}(k)$.)

Remarque 2.7 - On dispose aussi de la variante stable du foncteur «motif proche ». Elle est notée $\Psi_{\varpi}: \mathbf{D M}(F) \longrightarrow \mathbf{D M}(k)$ et elle est définie de la même manière. Il s'ensuit aussitôt un isomorphisme de commutation $\Psi_{\varpi} \circ \operatorname{Sus}_{T}^{0} \simeq \operatorname{Sus}_{T}^{0} \circ \Psi_{\varpi}$. (En effet, les foncteurs Rig* et $1^{*}$ commutent aux foncteurs de $T$-suspension infinie.) En utilisant le Lemme 2.3 on obtient alors le fait suivant. Soit $\left(\mathbf{E}_{n}\right)_{n \in \mathbb{N}}$ un $T$-spectre de complexes de préfaisceaux avec transferts sur $\mathrm{Sm} / F$, i.e., un objet de $\mathbf{D M}(F)$. On a alors un isomorphisme dans $\mathbf{D M}(k)$ :

$$
\Psi_{\varpi}(\mathbf{E})=\operatorname{hocolim}_{n \in \mathbb{N}} \operatorname{Sus}_{T}^{0}\left(\Psi_{\varpi}\left(\mathbf{E}_{n}\right)\right)(-n)[-2 n] .
$$

On laissera au lecteur le soin d'expliciter les morphismes de transition dans cette colimite.

Pour les catégories des motifs sans transferts $\mathbf{D A}(-)$ et $\mathbf{D A} \mathbf{A}^{\text {ét }}(-)$, on dispose d'une autre construction des foncteurs «motifs proches » qui ne passe pas par les catégories des motifs rigides (cf. [2, Chap. 3]). L'intérêt de cette construction est de se comparer plus facilement avec son analogue topologique via la réalisation de Betti (cf. [3]). Il sera donc utile de savoir le résultat suivant.

Lemme 2.8 - Il existe un carré commutatif à un isomorphisme canonique près :

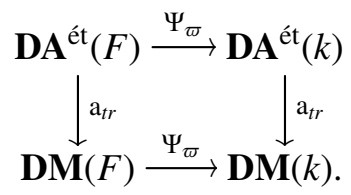

Demonstration On remarque d'abord que le carré

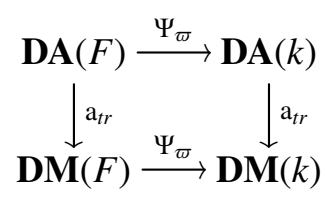

est commutatif à un isomorphisme canonique près. En effet, pour la catégorie $\mathbf{D A}(F)$ on peut définir un foncteur «motif proche » en passant par la catégorie $\operatorname{RigDA}(K)$ des motifs rigides grâce à [7, Scholie 1.3.26, (1)]. Avec cette définition, la commutation de (26) est tautologique et [7, Scholie 1.3.26, (2)] permet de conclure.

Pour obtenir la commutation du carré de l'énoncé, on utilise le fait que les flèches verticales dans (26) sont des foncteurs de localisation de Bousfield suivant les équivalences ét-locales. (En effet, rappelons-le, les flèches verticales dans (25) sont des équivalences de catégories.)

2.4. La réalisation de Betti. - Nous utiliserons la réalisation de Betti des motifs de Voevodsky construite dans [5, §1.1.2]. Nous rappellerons les grandes lignes de la construction dans l'espoir de préciser les notations.

Notons CpVar la catégorie des variétés analytiques complexes, lisses, dénombrables à l'infini et possédant un nombre fini de composantes connexes. On peut former la catégorie des correspondances finies AnCor ayant les mêmes objets que CpVar et où les morphismes sont donnés 
par les correspondances finies. Les foncteurs contravariants et additifs de AnCor dans la catégorie des $\mathbb{Q}$-espaces vectoriels sont appelés les préfaisceaux avec transferts sur CpVar. Ils forment une catégorie abélienne de Grothendieck qui sera notée PST(CpVar). Si $X \in \mathrm{CpVar}$, on note $\mathbb{Q}_{t r}(X)=\operatorname{Cor}(-, X) \otimes \mathbb{Q}$ le préfaisceau avec transferts représenté par $X$. Un faisceau avec transferts est un préfaisceau avec transferts dont la restriction à $\mathrm{CpVar}$ est un faisceau pour la topologie usuelle. Les faisceaux avec transferts forment une catégorie abélienne qui sera notée $\mathbf{S t r}(\mathrm{CpVar})$.

La catégorie $\mathbf{C p l ( P S T ( C p V a r ) ) ~ d e s ~ c o m p l e x e s ~ d e ~ p r e ́ f a i s c e a u x ~ a v e c ~ t r a n s f e r t s ~ p o s s e ̀ d e ~ u n e ~ s t r u c - ~}$ ture de modèles projective $\left(\mathbb{D}^{1}\right.$, usu)-locale obtenue par double localisation de Bousfield. La première localisation est topologique et fournit une catégorie homotopique équivalente à la catégorie dérivée $\mathbf{D}(\mathbf{S t r}(\mathrm{CpVar}))$. La seconde localisation est par rapport à la classe des morphismes $\mathbb{Q}_{t r}\left(\mathbb{D}^{1} \times X\right)[n] \longrightarrow \mathbb{Q}_{t r}(X)[n]$ avec $X \in \mathrm{CpVar}$ et $n \in \mathbb{Z}$. (Bien entendu, $\mathbb{D}^{1} \subset \mathbb{C}$ est le disque ouvert de centre 0 et de rayon 1.) La catégorie homotopique de la structure $\left(\mathbb{D}^{1}\right.$, usu)-locale est notée $\mathbf{A n D M}^{\text {eff }}$. Le résultat suivant est à la base de la construction de la réalisation de Betti.

Proposition 2.9 - Le foncteur $(-)_{\text {cst }}$ qui envoie un $\mathbb{Q}$-espace vectoriel $V$ sur le préfaisceau avec transferts constant $V_{c s t}: X \in \mathrm{CpVar} \rightsquigarrow V^{\pi_{0}(X)}$ induit une équivalence de catégories

$$
(-)_{c s t}: \mathbf{D}(\mathbb{Q}) \stackrel{\sim}{\longrightarrow} \mathbf{A n D M}^{\text {eff }} .
$$

Un quasi-inverse est donné par $\mathrm{R} \Gamma(-)$, le foncteur dérivé du foncteur "sections globales ».

On rappelle qu'on s'était fixé un plongement complexe $\sigma: k \hookrightarrow \mathbb{C}$. Étant donné $X \in \mathrm{Sm} / k$, on note $X^{a n}$ la variété analytique complexe associée, i.e., dont l'ensemble des points est donné par $X(\mathbb{C})$. Le foncteur $(-)^{a n}$ induit un foncteur triangulé

$$
\mathrm{An}^{*}: \mathbf{D M}^{e f f}(k) \longrightarrow \mathbf{A n D M}^{e f f} \text {. }
$$

Définition 2.10 - La réalisation de Betti est le foncteur $\mathrm{Bti}^{*}: \mathbf{D M}^{\text {eff }}(k) \longrightarrow \mathbf{D}(\mathbb{Q})$ donné par la composition de

$$
\mathbf{D M}^{e f f}(k) \stackrel{\mathrm{An}^{*}}{\longrightarrow} \mathbf{A n D M} \mathbf{M}^{e f f} \stackrel{\mathrm{R} \Gamma}{\longrightarrow} \mathbf{D}(\mathbb{Q}) .
$$

On notera $\mathrm{Bti}_{*}$ son adjoint à droite.

Remarque 2.11 - On dispose également de la variante stable AnDM de la catégorie AnDM ${ }^{\text {eff }}$. On a aussi les variantes sans transferts $\mathbf{A n D A}{ }^{\text {eff }}$ et AnDA. Toutes ces catégories sont équivalentes à $\mathbf{D}(\mathbb{Q})$, ce qui permet de définir des foncteurs de réalisation de Betti pour les catégories $\mathbf{D M}(k)$, $\mathbf{D A}^{\text {eff, ét }}(k)$ et $\mathbf{D} \mathbf{A}^{\text {ét }}(k)$. Ces foncteurs seront désignés par Bti*

Remarque 2.12 - Par construction, on a un isomorphisme $\mathrm{Bti}^{*} \circ \mathrm{Sus}_{T}^{0} \simeq \mathrm{Bti}^{*}$. En utilisant le Lemme 2.3 on obtient alors le fait suivant. Soit $\left(\mathbf{E}_{n}\right)_{n \in \mathbb{N}}$ un $T$-spectre de complexes de préfaisceaux avec transferts sur $\mathrm{Sm} / k$, i.e., un objet de $\mathbf{D M}(k)$. On a alors un isomorphisme dans $\mathbf{D}(\mathbb{Q})$ :

$$
\operatorname{Bti}^{*}(\mathbf{E})=\operatorname{hocolim}_{n \in \mathbb{N}} \operatorname{Bti}^{*}\left(\mathbf{E}_{n}\right) \otimes \operatorname{Bti}^{*}(\mathbb{Q}(1))^{\otimes-n}[-2 n] .
$$

On laissera au lecteur le soin d'expliciter les morphismes de transition dans cette colimite.

Pour la suite, nous devons aussi considérer la réalisation de Betti sur une base. Dans [3], on trouvera la construction d'une telle réalisation pour les catégories $\mathbf{D A} \mathbf{A}^{\text {eff }}(-)$ et $\mathbf{D A}(-)$. Dans [4, §2.1.2], nous avons montré que ces constructions s'étendent aussi aux catégories $\mathbf{D A}{ }^{\text {eff, ét }}(-)$ et

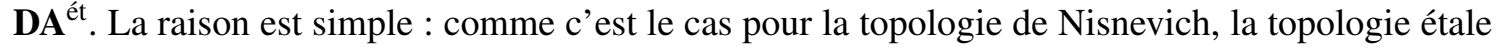
est moins fine que la topologie usuelle des variétés complexes. Si $X$ est un $k$-schéma (de type fini), les foncteurs de réalisation de Betti sont désignés par $\mathrm{Bti}_{X}^{*}$. Ils vont de $\mathbf{D A} \mathbf{A}^{e f f}(X), \mathbf{D A}(X)$, $\mathbf{D A}^{\text {eff, ét }}(X)$ et $\mathbf{D A}{ }^{\text {ét }}(X)$ dans $\mathbf{D}\left(X^{a n}\right)$, la catégorie dérivée des faisceaux de $\mathbb{Q}$-espaces vectoriels sur l'espace analytique complexe $X^{a n}$. Le principe de construction de ces foncteurs est le même que celui présenté ci-dessus. Nous renvoyons le lecteur à [3] et [4, \$2.1.2] pour plus de détails. Étant données les équivalences de catégories $\mathbf{D} A^{\text {eff, ét }}(X) \simeq \mathbf{D M}^{\text {eff }}(X)$ et $\mathbf{D} \mathbf{A}^{\text {ét }}(X) \simeq \mathbf{D M}(X)$ vraies pour $X$ un $k$-schéma lisse, on déduit aussitôt des foncteurs de réalisation de Betti

$$
\mathrm{Bti}^{*}: \mathbf{D M}^{e f f}(X) \longrightarrow \mathbf{D}\left(X^{a n}\right) \quad \text { et } \quad \mathrm{Bti}^{*}: \mathbf{D M}(X) \longrightarrow \mathbf{D}\left(X^{a n}\right) .
$$

On peut maintenant énoncer le théorème de compatibilité suivant. 
ThéORème 2.13 - Soit $U \subset \mathbb{A}_{k}^{1}$ un ouvert Zariski et notons $\Psi_{U}: \mathbf{D M}^{\text {eff }}(U) \longrightarrow \mathbf{D M}^{\text {eff }}(k)$ la composition de

$$
\mathbf{D M}^{e f f}(U) \stackrel{\varpi^{*}}{\longrightarrow} \mathbf{D M}^{\text {eff }}(F) \stackrel{\Psi_{\varpi}}{\longrightarrow} \mathbf{D M}^{\text {eff }}(k) .
$$

(Ci-dessus, $\varpi^{*}$ est le foncteur « image inverse » suivant le $F$-point $\varpi \in U(F) \subset \mathbf{G}_{m k}(F)$.)

Notons aussi $\Psi_{U^{a n}}: \mathbf{D}\left(U^{a n}\right) \longrightarrow \mathbf{D}(p t)=\mathbf{D}(\mathbb{Q})$ le foncteur "cycles proches » topologique. Alors, nous avons une face carrée

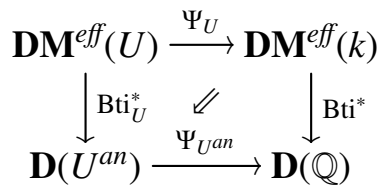

qui est inversible sur les motifs constructibles (alias compacts) dans $\mathbf{D M}^{\text {eff }}(U)$. La version stable est également vraie.

Demonstration D'après le Lemme 2.8, il suffit de démontrer l'énoncé analogue pour le foncteur «motif proche » $\Psi_{U}: \mathbf{D A}^{\text {ét }}(U) \longrightarrow \mathbf{D A}^{\text {ét }}(k)$ et la réalisation de Betti $\mathrm{Bti}^{*}: \mathbf{D A}^{\text {ét }}(-) \longrightarrow \mathbf{D}\left((-)^{a n}\right)$. Il s'agit alors de [3, Th. 4.9].

2.5. La réalisation de Betti tangentielle. - On arrive à une définition importante pour la suite de cet article. C'est la suivante.

Définition 2.14 - La réalisation de Betti tangentielle est le foncteur $\operatorname{TgB}^{*}: \mathbf{D M}^{e f f}(F) \longrightarrow \mathbf{D}(\mathbb{Q})$ donné par la composition de

$$
\mathbf{D M}^{e f f}(F) \stackrel{\Psi_{w}}{\longrightarrow} \mathbf{D M}^{e f f}(k) \stackrel{\mathrm{Bti}^{*}}{\longrightarrow} \mathbf{D}(\mathbb{Q}) .
$$

Son adjoint à droite sera noté $\mathrm{TgB}$.

Remarque 2.15 — Encore une fois, la réalisation de Betti tangentielle de la Définition 2.14 existe en plusieurs variantes. Il y a la variante stable, définie sur $\mathbf{D M}(F)$. Il y a aussi les variantes sans transferts définies sur $\mathbf{D A}(F)$ et $\mathbf{D} \mathbf{A}^{\text {ét }}(F)$. Tous ces foncteurs seront notés $\mathrm{TgB}$ *

Remarque 2.16 - Par construction, on a un isomorphisme $\operatorname{TgB}^{*} \circ \operatorname{Sus}_{T}^{0} \simeq \operatorname{TgB}^{*}$. En utilisant le Lemme 2.3 on obtient alors le fait suivant. Soit $\left(\mathbf{E}_{n}\right)_{n \in \mathbb{N}}$ un $T$-spectre de complexes de préfaisceaux avec transferts sur $\mathrm{Sm} / F$, i.e., un objet de $\mathbf{D M}(F)$. On a alors un isomorphisme dans $\mathbf{D}(\mathbb{Q})$ :

$$
\operatorname{TgB}^{*}(\mathbf{E})=\operatorname{hocolim}_{n \in \mathbb{N}} \operatorname{TgB}^{*}\left(\mathbf{E}_{n}\right) \otimes \operatorname{Bti}^{*}(\mathbb{Q}(1))^{\otimes-n}[-2 n] .
$$

On laissera au lecteur le soin d'expliciter les morphismes de transition dans cette colimite.

Soit $X$ un $k$-schéma lisse et notons $\operatorname{lisDM}^{e f f}(X) \subset \mathbf{D M}^{e f f}(X)$ la plus petite sous-catégorie triangulée, stable par sommes infinies et contenant les $X$-motifs effectifs qui deviennent fortement dualisables dans $\mathbf{D M}(X)$. Il s'agit de la version effective avec transferts de la catégorie $\operatorname{SmDA}(X)$ de [5, §2.4.1] qu'on notera ici lisDA $(X)$. Suivant la terminologie de [19], les objets de lisDM ${ }^{\text {eff }}(X)$ sont appelés des $X$-motifs lisses (effectifs). Toutefois, on attire l'attention du lecteur sur le fait que dans [19], les $X$-motifs lisses sont supposés constructibles alors que les nôtres ne le sont pas nécessairement.

Notons $\widehat{\mathbf{L S}}\left(X^{a n}\right) \subset \mathbf{S h v}\left(X^{a n}\right)$ la sous-catégorie abélienne ayant pour objets les faisceaux de $\mathbb{Q}$-espaces vectoriels sur $X^{a n}$ qui s'écrivent comme une union filtrante de sous-systèmes locaux. (Rappelons qu'un système local est simplement un faisceau localement constant ayant pour fibres des $\mathbb{Q}$-espaces vectoriels de dimension finie.) Notons aussi $\mathbf{D}_{\widehat{\mathrm{LS}}}\left(X^{a n}\right) \subset \mathbf{D}\left(X^{a n}\right)$ la sous-catégorie triangulée ayant pour objets les complexes de faisceaux $L$ tels que $\bigoplus_{n \in \mathbb{Z}} \mathrm{H}_{n}(L)$ est dans $\widehat{\mathbf{L S}}\left(X^{a n}\right)$. Avec ces notations, le foncteur de réalisation $\mathrm{Bti}_{X}^{*}: \mathbf{D M}^{e f f}(X) \longrightarrow \mathbf{D}\left(X^{a n}\right)$ envoie un motif lisse sur un objet de $\mathbf{D}_{\widehat{\mathrm{LS}}}\left(X^{a n}\right)$ (voir $\left.[\mathbf{5}, \S 2.4 .1]\right)$. Il induit donc un foncteur

$$
\mathrm{Bti}_{X}^{*}: \operatorname{lisDM}^{e f f}(X) \longrightarrow \mathbf{D}_{\widehat{\mathrm{LS}}}\left(X^{a n}\right) .
$$


Lemme 2.17 - Soit $U \subset \mathbb{A}_{k}^{1}$ un ouvert Zariski. On a un carré commutatif à un isomorphisme canonique près :

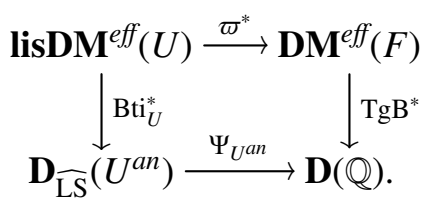

La version stable est également vraie.

Demonstration Ceci est une conséquence immédiate du Théorème 2.13 et du fait que le foncteur $\Psi_{U^{a n}}: \mathbf{D}\left(U^{a n}\right) \longrightarrow \mathbf{D}(\mathbb{Q})$, restreint à $\mathbf{D}_{\widehat{\mathrm{LS}}}\left(U^{a n}\right)$, commute aux sommes directes infinies.

THÉORÈME 2.18 - Soient $U \subset \mathbb{A}^{1} \backslash\{0\}$ un ouvert Zariski et $u \in U(k)$ un point rationnel. On fixe un lacet $\gamma:[0,1] \rightarrow \mathbb{C}$ différentiable en $0^{+}$tel que $\gamma(0)=0, \gamma^{\prime}(0)=1, \gamma(1)=u$ et $\left.\gamma(10,1]\right) \subset U(\mathbb{C})$. Il existe alors un isomorphisme (qui ne dépend que de $\gamma$ ) $\mathrm{t}_{\gamma}: \mathrm{TgB}^{*} \circ \varpi^{*} \simeq \mathrm{Bti}^{*} \circ u^{*}$ entre les compositions de

et de

$$
\operatorname{lisDM}^{e f f}(U) \stackrel{\varpi^{*}}{\longrightarrow} \mathbf{D M}^{\text {eff }}(F) \stackrel{\operatorname{TgB}^{*}}{\longrightarrow} \mathbf{D}(\mathbb{Q})
$$

$$
\operatorname{lisDM}^{e f f}(U) \stackrel{u^{*}}{\longrightarrow} \mathbf{D M}^{e f f}(k) \stackrel{\mathrm{Bti}^{*}}{\longrightarrow} \mathbf{D}(\mathbb{Q}) \text {. }
$$

De plus, si $p_{U}: U \longrightarrow \operatorname{Spec}(k)$ est le morphisme structural, on a un diagramme commutatif d'isomorphismes de foncteurs

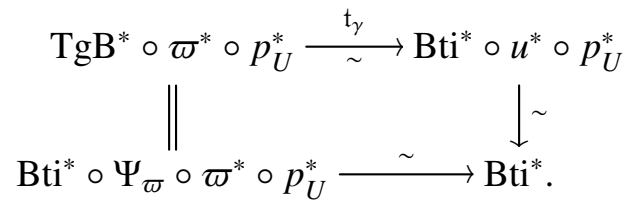

La version stable est également vraie.

Demonstration En effet, les compositions en question sont aussi les compositions de

$$
\operatorname{lisDM}^{e f f}(U) \stackrel{\mathrm{Bti}_{U}^{*}}{\longrightarrow} \mathbf{D}_{\widehat{\mathrm{LS}}}\left(U^{a n}\right) \stackrel{\Psi_{U^{a n}}}{\longrightarrow} \mathbf{D}(\mathbb{Q}) \quad \text { et } \quad \operatorname{lisDM}^{\text {eff }}(U) \stackrel{\mathrm{Bti}_{U}^{*}}{\longrightarrow} \mathbf{D}_{\widehat{\mathrm{LS}}}\left(U^{a n}\right) \stackrel{u^{*}}{\longrightarrow} \mathbf{D}(\mathbb{Q})
$$

(Pour la première composition on utilise le Lemme 2.17.) On est donc ramené à construire un isomorphisme entre les foncteurs $\Psi_{U^{a n}}, u^{*}: \mathbf{D}_{\widehat{\mathrm{LS}}}\left(U^{a n}\right) \longrightarrow \mathbf{D}(\mathbb{Q})$. Ceci est élémentaire mais nous devons revenir à la définition du foncteur « cycles proches » topologique. Notons $E=\mathbb{C} \sqcup\{(-\infty)\}$ munie de la topologie pour laquelle un système fondamental de voisinages de $(-\infty)$ est donné par les $\{z \in \mathbb{C} \mid \operatorname{Re}(z)<-N\} \sqcup\{(-\infty)\}$ avec $N \in \mathbb{N}$. On note $r: E \rightarrow \mathbb{C}$ la fonction continue donnée par $r(z)=e^{z}$ si $z \in \mathbb{C}$ et $r(-\infty)=0$. On a alors un diagramme commutatif

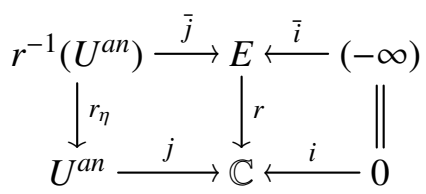

et $\Psi_{U^{a n}}=\bar{i}^{*} \bar{j}_{*} r_{\eta}^{*}$. Vu que exp : $\mathbb{C} \rightarrow \mathbb{C} \backslash\{0\}$ est un revêtement topologique, l'image inverse de $\gamma([0,1])$ est une union disjointe de courbes dans $\mathbb{C}$ qui s'envoient isomorphiquement sur $\gamma(] 0,1])$. Vu que $\gamma^{\prime}(0)=1$, il existe alors un unique relèvement $\left.\left.\bar{\gamma}:\right] 0,1\right] \rightarrow \mathbb{C}$ de $\gamma$ (i.e., tel que $\gamma=\exp \circ \bar{\gamma}$ ) avec $]-\infty, 0]$ asymptote à $\bar{\gamma}([0,1])$. On étend $\bar{\gamma}$ par continuité en posant $\bar{\gamma}(0)=(-\infty)$. On peut alors compléter le diagramme ci-dessus avec :

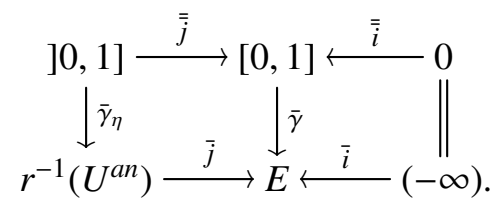


Pour tout $L \in \mathbf{D}_{\widehat{\mathrm{LS}}}\left(U^{a n}\right)$, on déduit un isomorphisme canonique

$$
\Psi_{U^{a n}}(L)=\bar{i}^{*} \overline{\bar{j}}_{*} r_{\eta}^{*}(L) \simeq \overline{\bar{i}}^{*} \overline{\bar{j}}_{*} \gamma_{\eta}^{*}(L)
$$

et $\gamma_{\eta}^{*}(L)$ est quasi-isomorphe à un faisceau constant. (Bien entendu, on a posé $\gamma_{\eta}=r_{\eta} \circ \bar{\gamma}_{\eta}$.) Il s'ensuit que $\overline{\bar{i}}^{*} \overline{\bar{j}_{*}} \gamma_{\eta}^{*}(L)$ est canoniquement isomorphe à $1^{*} \gamma_{\eta}^{*}(L)=u^{*}(L)$.

Remarque 2.19 - On peut adapter la preuve du Théorème 2.18 pour montrer que le foncteur $\mathrm{TgB}^{*}: \mathbf{D M}^{\text {eff }}(F) \longrightarrow \mathbf{D}(\mathbb{Q})$ est isomorphe (non canoniquement) au foncteur de réalisation de Betti associé à un plongement complexe $\sigma^{\prime}: F \hookrightarrow \mathbb{C}$ qui étend celui de $k$. On obtient alors la variante effective et avec transferts de [5, Prop. 2.20].

2.6. Un modèle explicite du foncteur $\mathrm{TgB}^{*}$, première partie. - L'objectif de ce paragraphe et du paragraphe suivant est de construire un modèle explicite du foncteur $\operatorname{TgB}^{*}$ dans l'esprit de $[4, \S 2.2 .4]$. Ce modèle constitue un ingrédient essentiel dans la preuve du Théorème 1.8. Comme dans $[4, \S 2.2 .4]$, on note $\overline{\mathbb{D}}_{\text {ét }}^{n}$ le pro-schéma des voisinages étales du polydisque fermé dans $\mathbb{A}_{k}^{n}$. Rappelons sa définition. On note $\mathcal{V}_{\text {ét }}\left(\overline{\mathbb{D}}^{n} / \mathbb{A}_{k}^{n}\right)$ la catégorie ayant pour objets les couples $(U, u)$ avec $U$ un $\mathbb{A}_{k}^{n}$-schéma étale et $u: \overline{\mathbb{D}}^{n} \longrightarrow U^{a n}$ un morphisme de (pro-)variétés complexes tel que le triangle

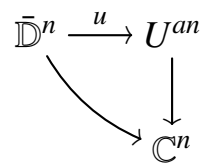

commute. (La flèche oblique ci-dessus est l'inclusion évidente.) D'après [4, Prop. 2.58], cette catégorie est cofiltrante. Le foncteur d'oubli $\overline{\mathbb{D}}_{\text {ét }}^{n}: \mathcal{V}_{\text {ét }}\left(\overline{\mathbb{D}}^{n} / \mathbb{A}_{k}^{n}\right) \longrightarrow \mathrm{Sm} / k$, qui à un couple $(U, u)$ associe le $k$-schéma $U$, définit un pro-schéma lisse et même étale au-dessus de $\mathbb{A}_{k}^{n}$. On voit immédiatement que la famille $\left\{\overline{\mathbb{D}}_{\text {ét }}^{n}\right\}_{n \in \mathbb{N}}$ s'étend d'une manière naturelle en un pro- $k$-schéma cocubique $\Sigma$-enrichi (au sens de [4, Déf. A.12]) qu'on notera $\overline{\mathbb{D}}_{\text {ét }}$.

DéfinItion 2.20 - Soit L un complexe de préfaisceaux avec transferts sur $\mathrm{Sm} / k$. Le complexe de chaînes semi-algébriques à valeurs dans L est

$$
\operatorname{Sg}_{\text {ét }}^{\mathbb{D}}(L)=\operatorname{Tot}\left(C\left(L\left(\overline{\mathbb{D}}_{\text {ét }}\right)\right)\right) \text {. }
$$

Ci-dessus, $\mathrm{C}(-)$ est le complexe simple associé à un objet cubique d'une catégorie additive (voir par exemple [4, Déf. A.4]) et Tot(-) est le complexe total associé à un multi-complexe (voir [4, $\S$ A.4] pour les conventions adoptées).

THÉorème 2.21 - Soit L un complexe de préfaisceaux avec transferts sur $\mathrm{Sm} / k$ (i.e., un objet de $\left.\mathbf{D M}^{\text {eff }}(k)\right)$. Alors, il existe un isomorphisme canonique :

$$
\operatorname{Bti}^{*}(L) \simeq \operatorname{Sg}_{\text {ét }}^{\mathbb{D}}(L) .
$$

Demonstration Il s'agit de la variante avec transferts de [4, Cor. 2.63]. Pour l'obtenir, il y a deux moyens de procéder : soit de remarquer que la preuve de [4, Cor. 2.63] se transporte littéralement au cas des préfaisceaux avec transferts, soit d'obtenir le théorème comme une conséquence de $[\mathbf{4}$, Cor. 2.63]. Expliquons rapidement l'argument si l'on opte pour la seconde possibilité. D'après Cisinski-Déglise, le foncteur $\mathrm{La}_{t r}: \mathbf{D A}^{e f f \text {, ét }}(k) \longrightarrow \mathbf{D M}^{e f f}(k)$ est une équivalence de catégories et son quasi-inverse est donné par $\operatorname{Ro}_{t r}$. Il s'ensuit que $\mathrm{Bti}^{*}(L) \simeq \mathrm{Bti}^{*} \operatorname{Ro}_{t r}(L)$. Par ailleurs, d'après [4, Lem. 2.111], le foncteur $\mathrm{o}_{t r}$ préserve les équivalences $\left(\mathbb{A}^{1}\right.$, ét)-locales. Il se dérive donc trivialement de sorte que $\operatorname{Ro}_{t r}(L) \simeq \mathrm{o}_{t r}(L)$. On applique maintenant [4, Cor. 2.63] pour conclure.

Étant donné un pro-schéma $X=\left\{X_{i}\right\}_{i}$, on note $\mathcal{O}(X)=\operatorname{colim}_{i} \mathcal{O}\left(X_{i}\right)$. Pour une utilisation future, nous rappelons le résultat suivant. 
Lemme 2.22 - Pour $n \in \mathbb{N}$, le pro-schéma $\overline{\mathbb{D}}_{\text {ét }}^{n}$ est affine et on a un isomorphisme canonique :

$$
\mathcal{O}\left(\overline{\mathbb{D}}_{\mathrm{et}}^{n}\right) \simeq \mathcal{O}_{k-a l g}\left(\overline{\mathbb{D}}^{n}\right) .
$$

Demonstration Il s'agit de [4, Prop. 2.58 et 2.102].

Nous expliquons maintenant un résultat analogue pour $\operatorname{Rig}^{*}: \mathbf{D M}^{e f f}(F) \longrightarrow \operatorname{RigDM}^{e f f}(K)$ auquel on pense comme à un foncteur de réalisation dans le monde analytique rigide. On commence par introduire quelques notations. Soit $X$ un $k$-schéma de type fini. On note $Q^{\text {rig }}(X)$ la fibre générique (de Raynaud) de la complétion formelle de $X \times_{k} K^{\circ}$ en sa fibre spéciale. Si $X=\operatorname{Spec}(A)$ est affine alors $Q^{r i g}(X)$ est le spectre maximal de la $K$-algèbre affinoïde $A[[\varpi]]\left[\varpi^{-1}\right]$. On note aussi $Q^{g e o}(X)=X \otimes_{k} F$. On dispose d'une inclusion évidente $Q^{r i g}(X) \hookrightarrow\left(Q^{g e o}(X)\right)^{a n r}$ naturelle en $X$ et qui dans le cas affine correspond au morphisme tautologique $A \otimes_{k} F \longrightarrow A[[\varpi]]\left[\varpi^{-1}\right]$.

Dans la suite, on considère uniquement le cas où $X$ est affine et on pose $A=\mathcal{O}(X)$. On définit un pro-schéma $Q_{\text {ét }}^{\text {rig }}(X)$ de la manière suivante. On note $\mathcal{V}_{\text {ét }}\left(Q^{r i g}(X) / Q^{\text {geo }}(X)\right)$ la catégorie ayant pour objets les couples $(U, u)$ avec $U$ un $Q^{g e o}(X)$-schéma étale et $u: Q^{r i g}(X) \longrightarrow U^{\text {anr }}$ un morphisme de variétés analytiques rigides tel que le triangle

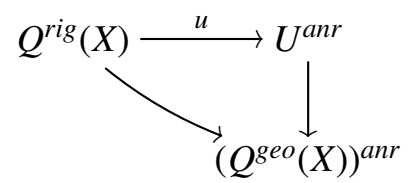

est commutatif. En adaptant la preuve de [4, Prop. 2.58], on montre que $\mathcal{V}_{\text {êt }}\left(Q^{r i g}(X) / Q^{g e o}(X)\right)$ est cofiltrante et que le foncteur $\operatorname{Spec}(-)$, restreint à l'ensemble ordonné des sous- $\left(A \otimes_{k} F\right)$-algèbres étales de $A[[\varpi]]\left[\varpi^{-1}\right]$, est cofinal. Le foncteur d'oubli $Q_{\text {êt }}^{r i g}(X): \mathcal{V}_{\text {ét }}\left(Q^{r i g}(X) / Q^{g e o}(X)\right) \longrightarrow \mathrm{Sm} / F$, qui envoie un couple $(U, u)$ sur le $F$-schéma $U$, définit un pro- $F$-schéma étale au-dessus de $Q^{g e o}(X)$.

DéfinITION 2.23 - Soit L un préfaisceau avec transferts sur $\mathrm{Sm} / F$. On note $\mathfrak{Q}_{*}(L)$ le préfaisceau avec transferts sur $\mathrm{Sm}^{\mathrm{af}} / k$ obtenu en posant $\mathfrak{Q}_{*}(L)(X)=L\left(Q_{\mathrm{et}}^{\text {rig }}(X)\right)$ pour tout $X \in \mathrm{Sm}^{\mathrm{af}} / k$. (Bien entendu, $\mathrm{Sm}^{\mathrm{af}}$ / $k$ est la catégorie des $k$-schémas affines et lisses.)

On peut maintenant énoncer le résultat suivant.

Théorème 2.24 - Soit L un complexe de préfaisceaux avec transferts sur $\mathrm{Sm} / F$. Alors, le complexe de préfaisceaux avec transferts $\mathfrak{Q}_{*}(L)$, vu comme objet de $\mathbf{D M}^{\text {eff }}(k)$, est canoniquement isomorphe à l'image de L par le foncteur composé

$$
\chi: \mathbf{D M}^{\text {eff }}(F) \stackrel{\operatorname{Rig}^{*}}{\longrightarrow} \operatorname{RigDM}^{\text {eff }}(K) \simeq \mathbf{q u D M}^{\text {eff }}(k) \hookrightarrow \mathbf{D M}^{\text {eff }}\left(\mathbf{G}_{\boldsymbol{m} k}\right) \stackrel{q_{*}}{\longrightarrow} \mathbf{D M}^{\text {eff }}(k) .
$$

Ci-dessus, $q: \mathbf{G}_{\boldsymbol{m} k} \longrightarrow \mathrm{Spec}(k)$ est la projection structurale.

Demonstration L'énoncé ci-dessus découle des résultats de [7, Chap. 2]. Expliquons comment l'obtenir. Nous divisons l'argument en plusieurs étapes.

Étape 1 : Considérons le foncteur

$$
\text { Rig* }^{*}: \mathbf{P S T}(\mathrm{Sm} / F) \longrightarrow \mathbf{P S T}(\mathrm{SmRig} / K)
$$

qui envoie $\mathbb{Q}_{t r}(X)$, pour $X \in \mathrm{Sm} / F$, sur $\mathbb{Q}_{t r}\left(X^{a n r}\right)$. (Précisons que le foncteur Rig* dans (38) est le foncteur dérivé à gauche du foncteur Rig $^{*}$ ci-dessus, i.e., on a Rig* ${ }^{*} \mathrm{LRig}^{*}$.) Étant donnés un $k$-affinoïde lisse $B$ et un préfaisceau avec transferts $E$ sur $\mathrm{Sm} / F$, on a

$$
\operatorname{Rig}^{*} E(B)=\underset{A \subset \mathcal{O}(B)}{\operatorname{colim}} E(\operatorname{Spec}(A))
$$

où $A$ parcourt l'ensemble filtrant des sous- $F$-algèbres lisses de $\mathcal{O}(B)$. (Voir la démonstration de [7, Lem. 2.5.20].) On en déduit aussitôt que le foncteur Rig $^{*}$ envoie les quasi-isomorphismes de complexes de préfaisceaux sur des équivalences ét-locales. Étant donné qu'il est de Quillen à gauche, ce foncteur envoie aussi une équivalence $\left(\mathbb{A}^{1}\right.$, ét)-locale sur une équivalence ( $\mathbb{B}^{1}$, ét)-locale. Il se dérive donc trivialement de sorte que $\operatorname{Rig}^{*}(L)=\operatorname{Rig}^{*}(L)$. 
Étape 2 : Choisissons un endofoncteur de ( $\mathbb{B}^{1}$, ét)-localisation $\operatorname{Loc}_{\mathbb{B}^{1} \text {,ét }}$ de $\operatorname{Cpl}(\mathbf{P S T}(\operatorname{SmRig} / K))$. On a alors une équivalence $\left(\mathbb{B}^{1}\right.$, ét)-locale

$$
\operatorname{Rig}^{*}(L) \longrightarrow \operatorname{Loc}_{\mathbb{B}^{1}, \text { ét }}\left(\operatorname{Rig}^{*}(L)\right)
$$

et le membre de droite est projectivement $\left(\mathbb{B}^{1}\right.$, ét)-fibrant. La composition de

$$
\operatorname{RigDM}^{e f f}(K) \simeq \mathbf{q u D M}^{e f f}(k) \hookrightarrow \mathbf{D M}^{e f f}\left(\mathbf{G}_{\boldsymbol{m} k}\right) \stackrel{q_{*}}{\longrightarrow} \mathbf{D M}^{e f f}(k)
$$

est l'adjoint à droite de la composition de

$$
\mathbf{D M}^{e f f}(k) \stackrel{(F / k)^{*}}{\longrightarrow} \mathbf{D M}^{e f f}(F) \stackrel{\mathrm{Rig}^{*}}{\longrightarrow} \mathbf{R i g D M}^{e f f}(K) .
$$

Il s'ensuit que (42) est isomorphe à $(F / k)_{*} \circ \operatorname{Rig}_{*}$ qui n'est autre que le foncteur dérivé du foncteur « image directe » suivant $\left(Q^{\text {geo }}(-)\right)^{a n r}: \operatorname{Cor}(k) \longrightarrow \operatorname{RigCor}(K)$. (Rappellons que $Q^{\text {geo }}(X)=X \otimes_{k}$ $F$.) Il s'ensuit que $\chi(L)$ est le complexe de préfaisceaux avec transferts donné par

$$
\chi(L)(X)=\operatorname{Loc}_{\mathbb{B}^{1}, \text { ét }}\left(\operatorname{Rig}^{*}(L)\right)\left(\left(Q^{g e o}(X)\right)^{a n r}\right)
$$

pour tout $X \in \mathrm{Sm} / k$.

Étape 3 : On a un morphisme canonique $Q^{\text {rig }}(X) \longrightarrow\left(Q^{\text {geo }}(X)\right)^{a n r}$ qui induit une équivalence $\left(\mathbb{B}^{1}\right.$, ét)-locale sur les motifs associés par [7, Th. 1.3.11]. Il en découle que le morphisme

$$
\operatorname{Loc}_{\mathbb{B}^{1}, \text { ét }}\left(\operatorname{Rig}^{*}(L)\right)\left(\left(Q^{g e o}(X)\right)^{a n r}\right) \longrightarrow \operatorname{Loc}_{\mathbb{B}^{1}, \text { ét }}\left(\operatorname{Rig}^{*}(L)\right)\left(Q^{r i g}(X)\right)
$$

est un quasi-isomorphisme. On obtient ainsi un isomorphisme

$$
\chi(L)(-) \simeq \operatorname{Loc}_{\mathbb{B}^{1}, \text { ét }}\left(\operatorname{Rig}^{*}(L)\right)\left(Q^{r i g}(-)\right)
$$

dans la catégorie dérivée $\mathbf{D}(\mathbf{P S T}(\mathrm{Sm} / k))$.

Étape 4 : On rappelle à présent un des résultats principaux de [7, Chap. 2]. Le $n$-ième simplexe analytique rigide est $\Delta_{r i g, K}^{n}=\operatorname{Spm}\left(K\left\{t_{0}, \cdots, t_{n}\right\} /\left(1-\sum_{i=0}^{n} t_{i}\right)\right)$. Lorsque $n$ varie, ces simplexes forment un $K$-affinoïde cosimplicial $\Delta_{\text {rig, } K}$. On pose alors $\underline{\mathrm{Sg}^{\mathbb{B}}}(-)=\operatorname{Tot}\left(\mathrm{C}\left(\underline{\operatorname{hom}}\left(\Delta_{\text {rig, },},-\right)\right)\right)$. C'est un endofoncteur de la catégorie des complexes de préfaisceaux avec transferts sur $\mathrm{SmRig} / K$. D'après [7, Th. 2.5.32], le morphisme canonique

$$
\operatorname{Rig}^{*}(L) \longrightarrow \underline{\operatorname{Sg}}^{\mathbb{B}}\left(\operatorname{Rig}^{*}(L)\right)
$$

est une équivalence $\left(\mathbb{B}^{1}\right.$, ét)-locale et le membre de droite est $\mathbb{B}^{1}$-local. Ainsi, si Locét est un endofoncteur de ét-localisation de $\mathbf{C p l}(\mathbf{P S T}(\operatorname{SmRig} / K))$, le complexe $\operatorname{Loc}_{\text {ét }}\left(\underline{\operatorname{Sg}}^{\mathbb{B}}\left(\operatorname{Rig}^{*}(L)\right)\right)$ est projectivement ( $\mathbb{B}^{1}$, ét)-fibrant. Autrement dit, le morphisme (41) se factorise de la manière suivante

$$
\operatorname{Rig}^{*}(L) \longrightarrow \underline{\mathrm{Sg}}^{\mathbb{B}}\left(\operatorname{Rig}^{*}(L)\right) \longrightarrow \operatorname{Loc}_{\text {ét }} \circ \underline{\mathrm{Sg}}^{\mathbb{B}}\left(\operatorname{Rig}^{*}(L)\right) .
$$

De plus, la seconde flèche ci-dessus est une équivalence ét-locale.

Étape 5 : D'après l'étape précédente, on déduit de (44) un isomorphisme

$$
\chi(L)(-) \simeq \text { Locét } \circ \underline{\operatorname{Sg}}^{\mathbb{B}}\left(\operatorname{Rig}^{*}(L)\right)\left(Q^{\text {rig }}(-)\right)
$$

dans la catégorie dérivée $\mathbf{D}(\mathbf{P S T}(\operatorname{SmRig} / K))$. Pour tout complexe de préfaisceaux avec transferts $E$ sur SmRig/K, on a

$$
\left(\underline{\mathrm{Sg}}^{\mathbb{B}}(E)\right)\left(Q^{r i g}(-)\right)=\underline{\mathrm{Sg}}^{\mathbb{A}}\left(E\left(Q^{r i g}(-)\right)\right)
$$

où $\underline{\operatorname{Sg}}^{\mathbb{A}}(-)=\operatorname{Tot}\left(\mathrm{C}\left(\underline{\operatorname{hom}}\left(\Delta_{k},-\right)\right)\right)$ est l'endofoncteur « complexe de Suslin-Voevodsky ». (Bien entendu, $\Delta_{k}$ est le schéma cosimplicial donné par $\Delta_{k}^{n}=\operatorname{Spec}\left(k\left[t_{0}, \cdots, t_{n}\right] /\left(1-\sum_{i=0}^{n} t_{i}\right)\right)$.) La trans-

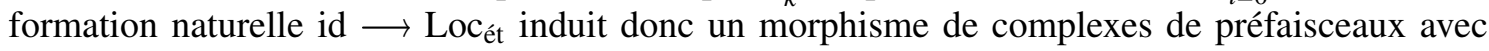
transferts sur $\mathrm{Sm} / k$ :

$$
\underline{\operatorname{Sg}}^{\mathbb{A}}\left(\operatorname{Rig}^{*}(L)\left(Q^{r i g}(-)\right)\right) \longrightarrow \operatorname{Loc}_{\text {ét }} \circ \underline{\operatorname{Sg}}^{\mathbb{B}}\left(\operatorname{Rig}^{*}(L)\right)\left(Q^{r i g}(-)\right) .
$$

Étape 6 : Le but ici est de montrer que (48) est une équivalence ét-locale. On montrera même que c'est une équivalence Nis-locale (ce qui est égal puisqu'on travaille avec transferts et à coefficients rationnels). 
Les deux membres de (48) sont $\mathbb{A}^{1}$-locaux. Pour le membre de gauche, ceci découle d'un théorème de Voevodsky [20, Cor. 14.9]. Pour le membre de droite, ceci découle du fait qu'il est quasi-isomorphe (en tant que complexe de préfaisceaux) à $(F / k)_{*} \circ \operatorname{Rig}_{*}\left(\operatorname{Loc}_{\mathbb{B}^{1} \text {,ét }}\left(\operatorname{Rig}^{*}(L)\right)\right)$, que $(F / k)_{*} \circ R i g_{*}$ est de Quillen à droite, et que $\operatorname{Loc}_{\mathbb{B}^{1} \text {, ét }}\left(\operatorname{Rig}^{*}(L)\right)$ est fibrant pour la structure projective $\left(\mathbb{B}^{1}\right.$, ét)-locale. Par un fait bien connu, il suffit donc de montrer que (48) induit un isomorphisme sur les points génériques des $k$-schémas lisses. (Utiliser par exemple [20, Lem. 22.8].)

Soit $X$ un $k$-schéma lisse et irréductible. Pour tout recouvrement Nisnevich $\left\{V_{i} \longrightarrow Q^{r i g}(X)\right\}_{i \in I}$, on peut trouver un ouvert non vide $U \subset X, i_{0} \in I$ et un triangle commutatif

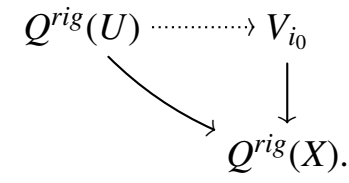

(Ceci est une conséquence facile de [7, Th. 1.2.10].) Une équivalence ét-locale $M \longrightarrow N$ de complexes de préfaisceaux avec transferts sur SmRig/ $K$ est aussi une équivalence Nis-locale (à cause des coefficients rationnels), et il découle de ce qui vient d'être dit que le morphisme canonique

$$
\operatorname{colim}_{\emptyset \neq U \subset X} M\left(Q^{r i g}(U)\right) \longrightarrow \operatorname{colim}_{\emptyset \neq U \subset X} N\left(Q^{r i g}(U)\right)
$$

est un quasi-isomorphisme. En appliquant ceci à $\underline{\operatorname{Sg}}^{\mathbb{B}}\left(\operatorname{Rig}^{*}(L)\right) \longrightarrow \operatorname{Loc}_{\text {ét }}\left(\underline{\operatorname{Sg}}^{\mathbb{B}}\left(\operatorname{Rig}^{*}(L)\right)\right)$ et en utilisant l'identification (47), on obtient le résultat recherché.

Étape 7 : D'après l'isomorphisme (46) et l'étape précédente, on a un isomorphisme dans $\mathbf{D M}^{e f f}(k)$ :

$$
\underline{\operatorname{Sg}^{\mathbb{A}}}\left(\left(\operatorname{Rig}^{*} L\right)\left(Q^{r i g}(-)\right)\right) \simeq \chi(L) .
$$

Par ailleurs, on dispose d'un morphisme

$$
\underline{\operatorname{Sg}}^{\mathbb{A}} Q_{*}(L)=\underline{\operatorname{Sg}}{ }^{\mathbb{A}}\left(L\left(Q_{\mathrm{et}}^{r i g}(-)\right)\right) \longrightarrow \underline{\operatorname{Sg}}{ }^{\mathbb{A}}\left(\left(\operatorname{Rig}^{*} L\right)\left(Q^{r i g}(-)\right)\right) .
$$

On montrera que c'est un quasi-isomorphisme de préfaisceaux sur $\mathrm{Sm}^{\mathrm{af}} / k$. Les deux membres préservent les quasi-isomorphismes de complexes de préfaisceaux avec transferts. (Pour le second membre, on utilisera la formule (40).) Un argument standard permet alors de se ramener au cas où $L=\mathbb{Q}_{t r}(W)$ avec $W \in \mathrm{Sm} / F$. Le résultat recherché découle alors de [7, Th. 2.4.16]. En effet, avec les notations de [7, Not. 2.4.14 et 2.4.15], si $U$ est un $k$-schéma affine et lisse, le complexe $\underline{\operatorname{Sg}}^{\mathbb{A}}\left(L\left(Q_{\text {ét }}^{r i g}(U)\right)\right)$, avec $L=\mathbb{Q}_{t r}(W)$, est égal à $\underline{\operatorname{Cor}}_{B}\left(\mathscr{V}\left(B / B_{0}\right), W \times_{k} B_{0}\right)$, avec $B_{0}=Q_{\text {êt }}^{r i g}(U)$ (en fait la limite projective de ce pro-schéma affine) et $B=Q^{\text {rig }}(U)$. (Il s'agit simplement de remarquer que $\mathscr{V}\left(\Delta_{r i g, B}^{n} / \Delta_{B_{0}}^{n}\right)$ est le pro-objet $Q_{\mathrm{et}}^{r i g}\left(\Delta_{U}^{n}\right)$.) D' autre part, le complexe $\underline{\operatorname{Sg}}^{\mathbb{A}}\left(\left(R i g^{*} L\right)\left(Q^{r i g}(U)\right)\right)$ est égal à $\underline{\mathbf{C o r}}_{B_{0}}\left(B, W \times_{k} B_{0}\right)$. Ceci termine la preuve du théorème.

Remarque 2.25 — Dans l'énoncé du Théorème 2.24, le symbole attribué au foncteur composé (38) n'était pas arbitraire. En effet, d'après [7, Scholie 1.3.26, (2)], la variante stable et sans transferts de ce foncteur composé s'identifie au foncteur $\chi_{\mathrm{id}}=i^{*} j_{*}$ avec $j: \operatorname{Spec}(F) \hookrightarrow \mathbb{A}_{k}^{1}$ et $i: \operatorname{Spec}(k) \hookrightarrow \mathbb{A}_{k}^{1}$ les inclusions évidentes ; ce dernier étant un constituant du système de spécialisation canonique $\chi$ de [2, Ex. 3.1.4].

On note le résultat suivant (cf. le Lemme 2.22).

Lemme 2.26 - Soit $X=\operatorname{Spec}(A)$ un k-schéma affine, lisse et connexe. Alors, $\mathcal{O}\left(Q_{\mathrm{ett}}^{\text {rig }}(X)\right)$ est canoniquement isomorphe à la sous-algèbre de $A[[\varpi]]\left[\varpi^{-1}\right]$ formées des éléments algébriques sur le corps des fractions de $A[\varpi]$.

Demonstration Il est facile d'adapter la preuve de [4, Prop. 2.102]. Les détails sont laissés au lecteur.

Pour $n \in \mathbb{N}$, on peut former le pro-schéma lisse $\overline{\mathbb{D}}_{\text {êt }}^{\dagger, n}=Q_{\text {êt }}^{r i g}\left(\overline{\mathbb{D}}_{\text {êt }}^{n}\right.$ ). (Il s'agit en fait d'un proobjet dans la catégorie des pro-schémas duquel on déduit un pro-schéma de la manière évidente.) Lorsque $n$ varie, on obtient un pro- $F$-schéma cocubique $\Sigma$-enrichi (au sens de [4, Déf. A.12]) qu'on notera $\overline{\mathbb{D}}_{\text {ét }}^{\dagger}$. ̀̀ ce stade, on a le corollaire suivant. 
Corollaire 2.27 - Soit L un complexe de préfaisceaux avec transferts sur $\mathrm{Sm} / F$. Alors, on a un isomorphisme canonique dans $\mathbf{D}(\mathbb{Q})$ :

$$
\operatorname{Tot}\left(\mathrm{C}\left(L\left(\overline{\mathbb{D}}_{\text {et }}^{\dagger}\right)\right)\right) \simeq \mathrm{Bti}^{*} \chi(L) .
$$

Demonstration Il s'agit de la conjonction des Théorèmes 2.21 et 2.24.

En utilisant les Lemmes 2.22 et 2.26, nous obtenons le résultat suivant que nous utiliserons plus tard.

Lemme 2.28 - Pour $n \in \mathbb{N}$, le pro-schéma $\overline{\mathbb{D}}_{\text {ét }}^{\dagger, n}$ est affine et on a un isomorphisme canonique :

$$
\mathcal{O}\left(\overline{\mathbb{D}}_{\text {ét }}^{\dagger, n}\right) \simeq \mathcal{O}_{k-a l g}^{\dagger}\left(\overline{\mathbb{D}}^{n}\right) .
$$

2.7. Un modèle explicite du foncteur $\mathrm{TgB}^{*}$, deuxième partie. - Le Corollaire 2.27 est bien joli, mais ce n'est pas encore le résultat qu'il nous faut. En effet, on aimerait plutôt avoir $\Psi_{\varpi}$ au lieu de $\chi$ dans la formule (50) et obtenir ainsi une formule pour la réalisation de Betti tangentielle (cf. la Définition 2.14).

Dans ce paragraphe nous nous efforcerons de remplacer $\chi$ par $\Psi_{\varpi}$ dans (50). Pour ce faire, nous serons malheureusement obligés de travailler stablement. Ainsi, la première moitié de ce paragraphe est consacrée à étendre les résultats du \$2.6 aux motifs stables.

Notation 2.29 — La réalisation de Betti du motif (effectif) $T=\mathbb{Z}_{t r}\left(\mathbb{P}_{k}^{1}, \infty\right)$ est isomorphe (non canoniquement) à $\mathbb{Z}[2]$. On fixe $\alpha \in \operatorname{Cor}_{k}\left(\overline{\mathbb{D}}_{\text {êt }}^{2}, \mathbb{P}_{k}^{1}\right)$ un cycle dans le complexe $\mathrm{C}\left(\operatorname{Cor}_{k}\left(\overline{\mathbb{D}}_{\text {ét }}, \mathbb{P}_{k}^{1}\right)\right)$ dont la classe d'homologie engendre $\mathrm{H}_{2}\left(\mathbb{P}^{1}(\mathbb{C})\right) \simeq \mathbb{Z}$. On supposera, ce qui est loisible, que la restriction de $\alpha$ au bord de $\overline{\mathbb{D}}_{\text {et }}^{2}$ est nulle.

ThÉORÈme $2.30-$ Soit $\mathbf{E}=\left(\mathbf{E}_{n}\right)_{n \in \mathbb{N}}$ un $T$-spectre de complexes de préfaisceaux avec transferts sur $\mathrm{Sm} / k$ (i.e., un objet de $\mathbf{D M}(k)$ ). Alors, il existe un isomorphisme canonique

$$
\mathrm{Bti}^{*}(\mathbf{E}) \simeq \text { hocolim }_{n \in \mathbb{N}} \operatorname{Sg}_{\text {ét }}^{\mathbb{D}}\left(\mathbf{E}_{n}\right)[-2 n] .
$$

Le morphisme de transition en $n \in \mathbb{N}$ est induit par la composition de :

$$
\mathbf{E}_{n}\left(\overline{\mathbb{D}}_{\text {êt }}^{m}\right) \longrightarrow \mathbf{E}_{n+1}\left(\left(\mathbb{P}_{k}^{1}, \infty\right) \times_{k} \overline{\mathbb{D}}_{\text {ét }}^{m}\right) \stackrel{\alpha^{*}}{\longrightarrow} \mathbf{E}_{n+1}\left(\overline{\mathbb{D}}_{\text {ét }}^{2+m}\right) .
$$

Demonstration Ceci découle du Théorème 2.21 et de l'isomorphisme (29).

On note $\chi: \mathbf{D M}(F) \longrightarrow \mathbf{D M}(k)$ la composition des versions stables des foncteurs de (38). Pour obtenir l'analogue stable du Théorème 2.24, on bute sur le fait que $\chi$ ne commute pas aux foncteurs de $T$-suspension infinie. Heureusement, on a une commutation partielle.

\section{Proposition 2.31 -}

(a) Il existe une transformation $\chi(N)(1) \longrightarrow \chi(N(1))$ naturelle en $N \in \mathbf{D M}^{\text {eff }}(F)$. Elle est inversible lorsque $N=M(1)$ pour un certain $M \in \mathbf{D M}^{\text {eff }}(F)$.

(b) Pour tout $s \in \mathbb{N}$, il existe une transformation $\operatorname{Sus}_{T}^{s} \circ \chi(N) \longrightarrow \chi \circ \operatorname{Sus}_{T}^{s}(N)$ naturelle en $N \in \mathbf{D M}^{e f f}(F)$. Elle est inversible lorsque $N=M(1)$ pour un certain $M \in \mathbf{D M}^{e f f}(F)$.

Demonstration Nous démontrons uniquement la seconde partie de l'énoncé. La première partie se démontre de la même manière.

D'après la construction des foncteurs $\chi$ et l'isomorphisme de commutation $\operatorname{Sus}_{T}^{s} \circ \operatorname{Rig}^{*} \simeq \operatorname{Rig}^{*} \circ$ $\operatorname{Sus}_{T}^{s}$, il suffit de construire une transformation naturelle

$$
\operatorname{Sus}_{T}^{s} \circ q_{*} \longrightarrow q_{*} \circ \operatorname{Sus}_{T}^{s}
$$

et de montrer qu'elle est inversible sur les objets de la forme $M(1)$ avec $M \in \mathbf{q u D M}^{\text {eff }}(k)$. La transformation naturelle en question est obtenue via l'adjonction $\left(q^{*}, q_{*}\right)$ à partir de l'isomorphisme de commutation $\operatorname{Sus}_{T}^{s} \circ q^{*} \simeq q^{*} \circ \operatorname{Sus}_{T}^{s}$. Par ailleurs, vu la construction de $\mathbf{q u D M}^{e f f}(k)$, on peut 
supposer que $M=e_{r \sharp} q^{*} M_{0}$ avec $M_{0} \in \mathbf{D M}^{\text {eff }}(k)$ et $e_{r}$ le morphisme d'élévation à la puissance $r \in \mathbb{N} \backslash\{0\}$. Vu que le foncteur $q_{*}$ est un facteur direct de $q_{*} e_{r}^{*}$, il suffit de montrer que le morphisme canonique

$$
\operatorname{Sus}_{T}^{s} q_{*} e_{r}^{*}\left(e_{r \sharp} q^{*} M_{0}(1)\right) \longrightarrow q_{*} e_{r}^{*} \operatorname{Sus}_{T}^{s}\left(e_{r \sharp} q^{*} M_{0}(1)\right)
$$

est inversible. Puisque $\operatorname{Sus}_{T}^{s}$ commute aux foncteurs $e_{r \sharp}$ et $q^{*}$, il suffit donc de montrer que

$$
\operatorname{Sus}_{T}^{s}\left(q_{*} e_{r}^{*} e_{r \sharp} q^{*} M_{0}(1)\right) \longrightarrow q_{*} e_{r}^{*} e_{r \sharp} q^{*}\left(\operatorname{Sus}_{T}^{s} M_{0}(1)\right)
$$

est inversible. Or, $e_{r}^{*} e_{r \sharp} q^{*} A=\bigoplus_{\xi \in \mu_{r}(k)} q^{*} A$ (du moins si $k$ contient les $r$-ièmes racines de l'unité, ce que l'on peut supposer dans cette preuve sans restreindre la généralité). Il est donc suffisant de montrer que

$$
\operatorname{Sus}_{T}^{s}\left(q_{*} q^{*} M_{0}(1)\right) \longrightarrow q_{*} q^{*}\left(\operatorname{Sus}_{T}^{s} M_{0}(1)\right)
$$

est inversible. Or, $q_{*} q^{*} A=A \oplus \underline{\mathcal{H} o m}(\mathbb{Q}(1)[1], A)$. Le résultat recherché est maintenant une conséquence du « cancellation theorem » de Voevodsky [23].

ThéORÈmE $2.32-$ Soit $\mathbf{E}=\left(\mathbf{E}_{n}\right)_{n \in \mathbb{N}}$ un T-spectre de complexes de préfaisceaux avec transferts sur $\mathrm{Sm} / F$. Alors, le T-spectre $\mathfrak{Q}_{*}(\mathbf{E})$, vu comme objet de $\mathbf{D M}(k)$, est canoniquement isomorphe à $\chi(\mathbf{E})$.

Demonstration On définit un morphisme de $T$-spectres $\mathfrak{Q}_{*}(\mathbf{E}) \longrightarrow \chi(\mathbf{E})$ dans $\mathbf{D M}(k)$ comme dans la preuve du Théorème 2.24. Il s'agit de prouver que ce morphisme est inversible. Pour cela, on supposera que $\mathbf{E}$ est projectivement cofibrant niveau par niveau. Ceci ne restreint pas la généralité puisque $\mathfrak{Q}_{*}$ préserve les quasi-isomorphismes de complexes de préfaisceaux.

Pour $s \in \mathbb{N}$, on note $\mathbf{E}^{(s)}=\left(\mathbf{E}_{n}^{(s)}\right)_{n \in \mathbb{N}}$ le $T$-spectre défini par :

$$
\mathbf{E}_{n}^{(s)}=\left\{\begin{array}{ccc}
\mathbf{E}_{n} & \text { si } & n \leq s \\
T^{\otimes n-s} \otimes \mathbf{E}_{s} & \text { si } & n \geq s .
\end{array}\right.
$$

On a des morphismes canoniques $\mathbf{E}^{(s)} \longrightarrow \mathbf{E}^{(s+1)}$ et $\mathbf{E} \simeq$ colim $_{s \in \mathbb{N}} \mathbf{E}^{(s)}$. Il est donc suffisant de traiter le cas des $T$-spectres $\mathbf{E}^{(s)}$. Par ailleurs, on dispose d'un morphisme $\operatorname{Sus}_{T}^{s}\left(\mathbf{E}_{s}\right) \longrightarrow \mathbf{E}^{(s)}$ qui induit un isomorphisme à partir du niveau $s$. En particulier, ce morphisme, ainsi que celui déduit par application de $\mathfrak{Q}_{*}$, sont des équivalences stables. Au final, il est donc suffisant de traiter le cas du $T$-spectre $\operatorname{Sus}_{T}^{s}\left(\mathbf{E}_{s}\right)$.

Pour la suite de la preuve, on note $M$ au lieu de $\mathbf{E}_{s}$. On dispose d'un morphisme évident

$$
w: \operatorname{Sus}_{T}^{s+1}(T \otimes M) \longrightarrow \operatorname{Sus}_{T}^{s}(M)
$$

qui est inversible à partir du niveau $s+1$. C'est donc une équivalence stable ainsi que $\mathfrak{Q}_{*}(w)$. Il est alors suffisant de traiter le cas de $\operatorname{Sus}_{T}^{s+1}(T \otimes M)$. Puisque $T \otimes M \simeq M(1)$ [2], il suffit de considérer le cas du $T$-spectre $\operatorname{Sus}_{T}^{s+1}(M(1))$.

Le $T$-spectre $\mathfrak{Q}_{*}\left(\operatorname{Sus}_{T}^{s+1}(M(1))\right)$ est donné en niveau $n \geq s+1$ par $\mathfrak{Q}_{*}(M(1)(n-s-1)[2 n-$ $2 s-2]$ ). D'après le Théorème 2.24 et la Proposition 2.31, (a), on a un isomorphisme canonique $\mathfrak{Q}_{*}(M(1))(n-s-1)[2 n-2 s-2] \simeq \mathfrak{Q}_{*}(M(1)(n-s-1)[2 n-2 s-2])$. Il s'ensuit que le $T$ spectre $\mathfrak{Q}_{*}\left(\operatorname{Sus}_{T}^{s+1}(M(1))\right)$ est isomorphe dans $\mathbf{D M}(k)$ à $\operatorname{Sus}_{T}^{s+1} \mathfrak{Q}_{*}(M(1))$. La même chose s'applique à $\chi$ d'après la Proposition 2.31, (b), i.e., $\chi\left(\operatorname{Sus}_{T}^{s+1}(M(1))\right)$ est canoniquement isomorphe à $\operatorname{Sus}_{T}^{s+1}(\chi(M(1)))$. On conclut maintenant par une deuxième application du Théorème 2.24.

Corollaire 2.33 - Soit $\mathbf{E}=\left(\mathbf{E}_{n}\right)_{n \in \mathbb{N}}$ un T-spectre de complexes de préfaisceaux avec transferts sur $\mathrm{Sm} / F$. Il existe alors un isomorphisme canonique dans $\mathbf{D}(\mathbb{Q})$ :

$$
\operatorname{colim}_{n \in \mathbb{N}} \operatorname{Tot}\left(\mathbf{C}\left(\mathbf{E}_{n}\left(\overline{\mathbb{D}}_{\text {ét }}^{\dagger}\right)\right)\right)[-2 n] \simeq \mathrm{Bti}^{*} \chi(\mathbf{E}) .
$$

Le morphisme de transition en $n \in \mathbb{N}$ est induit par la composition de :

$$
\mathbf{E}_{n}\left(\overline{\mathbb{D}}_{\text {êt }}^{\dagger, m}\right) \longrightarrow \mathbf{E}_{n+1}\left(\left(\mathbb{P}_{F}^{1}, \infty\right) \times_{F} \overline{\mathbb{D}}_{\text {êt }}^{\dagger, m}\right) \stackrel{\alpha^{*}}{\longrightarrow} \mathbf{E}_{n+1}\left(\overline{\mathbb{D}}_{\text {êt }}^{\dagger, 2+m}\right) .
$$

Demonstration Il s'agit de la conjonction des Théorèmes 2.30 et 2.32. 
On explique maintenant comment déduire de (54) une formule similaire pour le foncteur $\operatorname{TgB}^{*}=$ $\mathrm{Bti}^{*} \Psi_{\varpi}$ tout en précisant l'endroit où l'utilisation de la catégorie des motifs stables est essentielle.

On aura besoin du diagramme de $\mathbf{G}_{\boldsymbol{m}}{ }_{k}$-schémas $\mathscr{R}$ introduit dans [2, Déf. 3.5.3]. La catégorie d'indices de $\mathscr{R}$ est $\Delta \times \mathbb{N}^{\times}$où $\Delta$ est la catégorie des simplexes et $\mathbb{N}^{\times}$est l'ensemble $\mathbb{N}-\{0\}$ ordonné par la relation opposée à celle de divisibilité. On a $\mathscr{R}(\underline{\mathbf{n}}, r)=\mathbf{G}_{\boldsymbol{m} k} \times_{k}\left(\mathbf{G}_{\boldsymbol{m} k}\right)^{n}$ et son morphisme structural (de $\mathbf{G}_{\boldsymbol{m}}$-schéma) est la composition de la projection sur le premier facteur avec le morphisme d'élévation à la puissance $r$ de $\mathbf{G}_{\boldsymbol{m} k}$. Autrement dit, $\mathscr{R}(\underline{\mathbf{n}}, r)=Q_{r}^{g m}\left(\left(\mathbf{G}_{\boldsymbol{m} k}\right)^{n}\right)$. Étant donnés des entiers non nuls $r$ et $d$, le morphisme $\mathscr{R}(\underline{\mathbf{n}}, r d) \longrightarrow \mathscr{R}(\underline{\mathbf{n}}, r)$ est l'élévation à la puissance $d$ sur chaque facteur. Le $k$-schéma cosimplicial $\mathscr{R}(-, r)$ ne dépend pas de $r \in \mathbb{N}^{\times}$. Son morphisme face $\mathrm{d}_{i}: \mathscr{R}(\underline{\mathbf{n}}, r) \longrightarrow \mathscr{R}(\underline{\mathbf{n}}+\mathbf{1}, r)$ est induit par l'immersion diagonale du $(i+1)$-ème facteur si $i \leq n$ et consiste en l'insertion d'un 1 au $(n+2)$-ème facteur si $i=n+1$. Ses morphismes de dégénérescence $\mathrm{s}_{i}$ sont donnés par la projection suivant le $(i+2)$-ème facteur.

Notons $(\theta, \mathrm{p})=\mathrm{p} \circ \theta: \mathscr{R} \longrightarrow \mathbf{G}_{\boldsymbol{m} k}$ la projection canonique, i.e., $\mathrm{p}$ est l'unique foncteur de $\Delta \times \mathbb{N}^{\times}$vers la catégorie finale et $\theta$ est le morphisme de $\left(\Delta \times \mathbb{N}^{\times}\right)$-diagrammes de schémas de $\mathscr{R}$ vers le diagramme constant $\left(\mathbf{G}_{\boldsymbol{m} k}, \boldsymbol{\Delta} \times \mathbb{N}^{\times}\right)$donné par les morphismes structuraux des $\mathbf{G}_{\boldsymbol{m} k^{-}}$ schémas. On a le résultat suivant. (Pour la signification des opérations ci-dessous nous renvoyons le lecteur à $[1, \S 2.4 .2]$.)

Proposition 2.34 - Il existe une transformation

$$
q_{*}\left(\mathrm{p}_{\sharp} \theta_{*} \theta^{*} \mathrm{p}^{*} M\right) \longrightarrow 1^{*} M
$$

naturelle en $M \in \mathbf{D M}^{\text {eff }}\left(\mathbf{G}_{\boldsymbol{m}_{k}}\right)$. Elle est inversible lorsque $M \in \mathbf{q u D M}^{\text {eff }}(k)$. L'analogue stable est aussi vrai. (Ci-dessus, $q: \mathbf{G}_{m_{k}} \longrightarrow \mathrm{Spec}(k)$ est le morphisme structural du k-schéma en groupe $\mathbf{G}_{\boldsymbol{m}_{k}}$ et $1 \in \mathbf{G}_{\boldsymbol{m}_{k}}(k)$ est sa section unité.)

Demonstration Pour construire (55), on remarque qu' on a un morphisme de $\left(\Delta \times \mathbb{N}^{\times}\right)$-diagrammes de schémas $u:\left(\operatorname{Spec}(k), \Delta \times \mathbb{N}^{\times}\right) \longrightarrow\left(\mathscr{R}, \Delta \times \mathbb{N}^{\times}\right)$donné par la section unité du tore $\left(\mathbf{G}_{\boldsymbol{m} k}\right)^{n+1}$ pour chaque $(\underline{\mathbf{n}}, r) \in \Delta \times \mathbb{N}^{\times}$. Il induit une transformation naturelle

$$
\mathrm{p}_{\sharp} \theta_{*} \theta^{*} \mathrm{p}^{*} \longrightarrow \mathrm{p}_{\sharp} 1_{*} 1^{*} \mathrm{p}^{*} \simeq 1_{*} 1^{*} .
$$

La transformation naturelle (55) s'en déduit en appliquant $q_{*}$ et en utilisant l'isomorphisme id $\simeq$ $q_{*} 1_{*}$. Pour montrer que la transformation naturelle ainsi construite est inversible lorsqu'on l'évalue en $M \in \mathbf{q u D M} \mathbf{M}^{e f f}(k)$, on peut se restreindre à un ensemble de générateurs. On peut donc supposer que $M=\mathbb{Q}_{t r}\left(Q_{r}^{g m}(X)\right)$ avec $r \in \mathbb{N}^{\times}$et $X \in \mathrm{Sm} / k$. Dans ce cas, on a $e_{r}^{*} M \simeq q^{*} M_{0}$ avec $e_{r}$ l'endomorphisme d'élévation à la puissance $r$ de $\mathbf{G}_{\boldsymbol{m} k}$ et $M_{0}=\mathbb{Q}_{t r}\left(X \times_{k} \mu_{r}\right)$. Par ailleurs, on a l'égalité $e_{r} \circ(\theta, \mathrm{p})=(\theta, \mathrm{p}) \circ \iota_{r}$ avec $\iota_{r}:(\mathscr{R}, \Delta \times \mathbb{N}) \longrightarrow(\mathscr{R}, \Delta \times \mathbb{N})$ l'endomorphisme induit par le foncteur $\iota_{r}$ donné par : $\underline{(\underline{\mathbf{n}}, d)} \rightsquigarrow(\underline{\mathbf{n}}, r d)$. On en déduit aussitôt un isomorphisme canonique

$$
\mathrm{p}_{\sharp} \theta_{*} \theta^{*} \mathrm{p}^{*} \simeq e_{r *} \mathrm{p}_{\sharp} \theta_{*} \theta^{*} \mathrm{p}^{*} e_{r}^{*} .
$$

(Utiliser le fait que $\iota_{r}$ est cofinal.) Il est donc suffisant de considérer le cas de $e_{r}^{*} M=q^{*} M_{0}$. Ainsi, on s'est ramené à montrer que le morphisme canonique

$$
q_{*} \mathrm{p}_{\sharp} \theta_{*} \theta^{*} \mathrm{p}^{*} q^{*} \longrightarrow q_{*} 1_{*} 1^{*} q^{*}
$$

est inversible. Ce morphisme admet une section donnée, à un isomorphisme près, par

$$
\mathrm{id} \simeq \mathrm{p}_{\sharp} \mathrm{p}^{*} \longrightarrow \mathrm{p}_{\sharp} q_{*} \theta_{*} \theta^{*} q^{*} \mathrm{p}^{*} .
$$

Or, pour $r \in \mathbb{N}^{\times}$fixé, le morphisme de $k$-schémas cosimpliciaux $(\mathscr{R}(-, r), \Delta) \longrightarrow(\operatorname{Spec}(k), \Delta)$ est une équivalence d'homotopie cosimpliciale par [2, Cor. 3.4.5]. Le résultat découle maintenant de [2, Cor. 3.4.12]. La version stable se démontre de la même manière.

Le résultat suivant est faux pour les motifs effectifs (voir l'Exemple 2.36 ci-dessous) et il en est de même du Théorème 2.39. C'est pour cette raison qu'on est obligé de travailler avec les $T$-spectres.

Corollaire 2.35 - Il existe un isomorphisme canonique

$$
\Psi_{\varpi} \simeq \chi \circ\left(\mathrm{p}_{\sharp}\left(\theta_{\varpi}\right)_{*}\left(\theta_{\varpi}\right)^{*} \mathrm{p}^{*}\right)
$$


entre foncteurs de $\mathbf{D M}(F)$ dans $\mathbf{D M}(k)$. Ci-dessus, $\left(\theta_{\varpi}, \mathrm{p}\right): \mathscr{R}_{\varpi}=\mathscr{R} \times_{\mathbf{G}_{m k}, \varpi} \operatorname{Spec}(F) \longrightarrow \operatorname{Spec}(F)$ est la projection sur le second facteur.

Demonstration En effet, d'après la Proposition 2.34, on a un isomorphisme canonique entre $\Psi_{\varpi}$ et la composition de

$$
\mathbf{D M}(F) \stackrel{\operatorname{Rig}^{*}}{\longrightarrow} \operatorname{RigDM}(K) \simeq \mathbf{q u D M}(k) \hookrightarrow \mathbf{D M}\left(\mathbf{G}_{\boldsymbol{m}_{k}}\right) \stackrel{\mathrm{p}_{\sharp} \theta_{*} \theta^{*} \mathbf{p}^{*}}{\longrightarrow} \mathbf{D M}\left(\mathbf{G}_{\boldsymbol{m}_{k}}\right) \stackrel{q_{*}}{\longrightarrow} \mathbf{D M}(k) .
$$

Par ailleurs, étant donné un $T$-spectre $\mathbf{E}=\left(\mathbf{E}_{n}\right)_{n \in \mathbb{N}}$ dans $\mathbf{D M}\left(\mathbf{G}_{\boldsymbol{m} k}\right)$, le $T$-spectre $\mathrm{p}_{\sharp} \theta_{*} \theta^{*} \mathrm{p}^{*}(\mathbf{E})$ est simplement la colimite homotopique du diagramme de $T$-spectres $\operatorname{Rhom}(\mathscr{R}, \mathbf{E})$. Si $\mathbf{E}$ est l'image d'un $T$-spectre $\mathbf{E}^{\prime} \in \mathbf{R i g D M}(K)$, alors la colimite homotopique de $\overline{\operatorname{Rhom}}(\mathscr{R}, \mathbf{E})$ est l'image de la colimite homotopique de $\underline{\operatorname{Rhom}}\left(\left(\mathscr{R} \times_{\mathbf{G}_{m k}} F\right)^{a n r}, \mathbf{E}^{\prime}\right)$. Or, pour les motifs stables, on a une commutation

$$
\operatorname{Rig}^{*} \circ \operatorname{Rhom}(X,-) \simeq \operatorname{Rhom}\left(X^{a n r},-\right) \circ \operatorname{Rig}^{*}
$$

pour tout $X \in \mathrm{Sm} / F$. (En effet, le motif stable $\mathrm{M}(X)$ de $X$ est fortement dualisable ce qui entraîne des isomorphismes canoniques $\operatorname{Rhom}(X,-) \simeq \mathrm{M}(X)^{\vee} \otimes-, \operatorname{Rhom}\left(X^{a n r},-\right) \simeq \mathrm{M}\left(X^{a n r}\right)^{\vee} \otimes-$ et

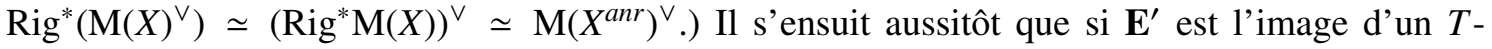
spectre $\mathbf{E}^{\prime \prime} \in \mathbf{D M}(F)$, alors la colimite homotopique de $\operatorname{Rhom}\left(\left(\mathscr{R} \times_{\mathbf{G}_{m k}} F\right)^{a n r}, \mathbf{E}^{\prime}\right)$ est l'image de la colimite homotopique de $\operatorname{Rhom}\left(\mathscr{R} \times_{\mathbf{G}_{m k}} F, \mathbf{E}^{\prime \prime}\right)$. Ceci se traduit par un carré commutatif à un isomorphisme canonique près :

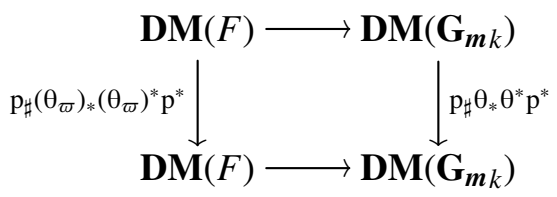

où les foncteurs horizontaux sont les composés des trois premiers foncteurs dans (56). Ceci permet de conclure.

Exemple 2.36 - On peut se convaincre que les foncteurs $\Psi_{\varpi}$ et $\chi \circ\left(p_{\sharp}\left(\theta_{\varpi}\right)_{*}\left(\theta_{\varpi}\right)^{*} p^{*}\right)$ ne sont pas isomorphes pour les motifs effectifs en regardant le cas du $\mathrm{H}_{1}$ motivique d'une $F$-courbe elliptique $\mathcal{E}$ à mauvaise réduction multiplicative. Ce motif effectif est donné par le complexe $\underline{\mathcal{E}}[-1]$ où $\underline{\mathcal{E}}$ est le faisceau avec transferts représenté par $\mathcal{E}$ (à torsion près). Puisque $\underline{\mathcal{E}}$ est birationnel, i.e.,

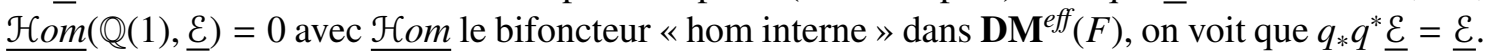
Il s'ensuit aussitôt que $\left(\mathrm{p}_{\sharp}\left(\theta_{\varpi}\right)_{*}\left(\theta_{\varpi}\right)^{*} \mathrm{p}^{*}\right) \underline{\mathcal{E}}=\underline{\mathcal{E}}$. Or, on peut calculer $\Psi_{\varpi}(\underline{\mathcal{E}}[-1])$ et $\chi(\underline{\mathcal{E}}[-1])$ : on trouve respectivement $\mathbb{Q}(0) \oplus \mathbb{Q}(1)$ et $\mathbb{Q}(0) \oplus \mathbb{Q}(1) \oplus \mathbb{Q}(0)[-1]$.

ThÉORÈme $2.37-$ Soit $\mathbf{E}=\left(\mathbf{E}_{n}\right)_{n \in \mathbb{N}}$ un T-spectre de complexes de préfaisceaux avec transferts sur $\mathrm{Sm} / F$. Alors, le T-spectre

$$
\operatorname{colim}_{r \in \mathbb{N}^{\times}} \mathfrak{Q}_{*}\left(\operatorname{Tot}\left(\mathrm{C}\left(\underline{\operatorname{hom}}\left(\mathscr{R}_{\varpi}(-, r), \mathbf{E}\right)\right)\right)\right),
$$

vu comme objet de $\mathbf{D M}(k)$, est canoniquement isomorphe à $\Psi_{\varpi}(\mathbf{E})$.

Demonstration C'est essentiellement la conjonction du Théorème 2.32 et du Corollaire 2.35. Nous devons seulement faire attention au fait que le foncteur $\left(\theta_{\varpi}\right)_{*}$ est par définition le foncteur dérivé du foncteur hom $\left(\mathscr{R}_{\varpi},-\right)$, alors qu'on ne suppose pas à priori que $\mathbf{E}$ est stablement $\left(\mathbb{A}^{1}\right.$, ét)-fibrant. Ceci ne pose pas de problème car les foncteurs $\underline{h o m}\left(\mathscr{R}_{\varpi}(\underline{\mathbf{n}}, r),-\right)$ préservent les équivalences $\left(\mathbb{A}^{1}\right.$, ét)-locales stables d'après le Lemme 2.38 ci-dessous.

Lemme 2.38 - Soient $F^{\prime} / F$ une extension finie et $U \subset \mathbb{A}_{F^{\prime}}^{1}$ un ouvert Zariski. Alors, le foncteur $\underline{\mathrm{hom}}(U,-)$ préserve les équivalences $\left(\mathbb{A}^{1}\right.$, ét)-locales stables.

Demonstration C'est une conséquence de certains travaux de Voevodsky. On montre d'abord que ce foncteur préserve les équivalences $\left(\mathbb{A}^{1}\right.$, ét)-locales entre complexes de préfaisceaux avec transferts. Soit $f: L \longrightarrow M$ un tel morphisme. Alors, $\mathrm{Sg}^{\mathbb{A}}(f)$ est aussi une équivalence ( $\mathbb{A}^{1}$, ét)locale, et les complexes $\underline{\operatorname{Sg}}^{\mathbb{A}}(L)$ et $\underline{\operatorname{Sg}}^{\mathbb{A}}(M)$ sont $\mathbb{A}^{1}$-locaux d'après Voevodsky [20]. (Ici, $\underline{\operatorname{Sg}}{ }^{\mathbb{A}}=$ 
$\operatorname{Tot}\left(\mathrm{C}\left(\underline{\operatorname{hom}}\left(\Delta_{F},-\right)\right)\right)$ désigne la construction de Suslin-Voevodsky.) Par ailleurs,

$$
\left.\underline{\operatorname{hom}}(U, L) \longrightarrow \underline{\operatorname{hom}}\left(U, \underline{\operatorname{Sg}^{\mathbb{A}}}(L)\right)=\underline{\operatorname{Sg}^{\mathbb{A}}} \underline{\operatorname{hom}}(U, L)\right)
$$

est une équivalence $\left(\mathbb{A}^{1}\right.$, ét)-locale, et pareillement pour $M$. Il est donc suffisant de montrer que le morphisme

$$
\underline{\operatorname{hom}}\left(U, \underline{\operatorname{Sg}^{\mathbb{A}}}(L)\right) \longrightarrow \underline{\operatorname{hom}}\left(U, \underline{\operatorname{Sg}^{\mathbb{A}}}(M)\right)
$$

est une équivalence ét-locale. Autrement dit, il faut montrer que

$$
\underline{\operatorname{hom}}\left(U, \mathrm{H}_{i} \underline{\operatorname{Sg}}^{\mathbb{A}}(L)\right) \longrightarrow \underline{\operatorname{hom}}\left(U, \mathrm{H}_{i} \underline{\operatorname{Sg}^{\mathbb{A}}}(M)\right)
$$

induit un isomorphisme sur les faisceaux associés pour tout $i \in \mathbb{Z}$. Ci-dessus $A=\mathrm{H}_{i} \underline{\operatorname{Sg}^{\mathbb{A}}}(L)$ et $B=\mathrm{H}_{i} \underline{\operatorname{Sg}^{\mathbb{A}}}(M)$ sont les préfaisceaux d'homologie.

Les deux membres de (58) sont des préfaisceaux avec transferts invariants par homotopie. Il suffit alors de montrer que (58) est inversible sur les points génériques des $F$-schémas lisses. (On utilise pour cela [20, Lem. 22.8].) Nous vérifierons ceci uniquement pour le corps $F$; la preuve en général est identique. D'après [20, Lect. 22], nous avons $A(U)=\mathrm{H}_{\mathrm{Nis}}^{0}(U, A)$ et pareillement pour $B$. Le résultat recherché découle donc du fait que $A \longrightarrow B$ induit un isomorphisme sur les faisceaux Nisnevich associés.

Le passage aux $T$-spectres est formel. Le lecteur pourra s'inspirer de l'argument utilisé dans [4, Lem. 2.112].

ThÉORÈme 2.39 - Soit $\mathbf{E}=\left(\mathbf{E}_{n}\right)_{n \in \mathbb{N}}$ un $T$-spectre de complexes de préfaisceaux avec transferts sur $\mathrm{Sm} / F$. Il existe alors un isomorphisme canonique dans $\mathbf{D}(\mathbb{Q})$ :

$$
\operatorname{TgB}^{*}(\mathbf{E}) \simeq \operatorname{colim}_{n \in \mathbb{N}} \operatorname{colim}_{r \in \mathbb{N}^{x}} \operatorname{Tot}\left(\mathrm{C}\left(\mathbf{E}_{n}\left(\overline{\mathbb{D}}_{\text {et }}^{\dagger} \times_{F} \mathscr{R}_{\varpi}(-, r)\right)\right)\right)[-2 n] .
$$

Le morphisme de transition en $n \in \mathbb{N}$ est induit par la composition de :

$$
\mathbf{E}_{n}\left(\overline{\mathbb{D}}_{\text {êt }}^{\dagger, m} \times_{F} \mathscr{R}_{\varpi}\right) \longrightarrow \mathbf{E}_{n+1}\left(\left(\mathbb{P}_{F}^{1}, \infty\right) \times_{F} \overline{\mathbb{D}}_{\text {êt }}^{\dagger, m} \times_{F} \mathscr{R}_{\varpi}\right) \stackrel{\alpha^{*}}{\longrightarrow} \mathbf{E}_{n+1}\left(\overline{\mathbb{D}}_{\text {êt }}^{\dagger, 2+m} \times_{F} \mathscr{R}_{\varpi}\right) .
$$

Demonstration C'est la conjonction des Théorèmes 2.30 et 2.37. On aurait pu aussi utiliser les Corollaires 2.33 et 2.35 (joints au Lemme 2.38).

Remarque 2.40 - On ignore si la version sans transferts du Théorème 2.39 est vraie. (La question a d'autant plus de sens que c'est bien le cas pour la version sans transferts du Théorème 2.30.) La preuve que nous avons présentée repose d'une manière essentielle sur des propriétés spéciales des préfaisceaux avec transferts invariants par homotopie (en géométrie algébrique et en géométrie rigide !) et notamment l'invariance par homotopie de la cohomologie de tels préfaisceaux. Toutefois, si $\mathbf{E}$ est un $T$-spectre de complexes de préfaisceaux (sans transferts) sur $\mathrm{Sm} / F$, la conclusion du Théorème 2.39 est vraie lorsque $\mathbf{E}$ est projectivement $\left(\mathbb{A}^{1}\right.$, ét)-fibrant niveau par niveau. En effet, dans ce cas $\mathbf{E}$ est quasi-isomorphe niveau par niveau à $\mathrm{Ro}_{t r} \mathrm{La}_{t r} \mathbf{E}$. (C'est une conséquence de l'équivalence de Cisinski-Déglise $\mathbf{D A}{ }^{e f f}$ ét $(F) \simeq \mathbf{D M}^{e f f}(F)$.) On utilise alors l'isomorphisme $\mathrm{TgB}^{*} \circ \mathrm{Ro}_{t r} \simeq \mathrm{TgB}^{*}$ pour conclure.

\section{Groupes de Galois motiviques et torseurs d'isomorphismes}

On garde les notations de la Section 2. Nous rappellerons d'abord la construction des groupes de Galois motiviques associés aux foncteurs de réalisation

$$
\mathrm{Bti}^{*}: \mathbf{D M}(k) \longrightarrow \mathbf{D}(\mathbb{Q}) \quad \text { et } \quad \operatorname{TgB}^{*}: \mathbf{D M}(F) \longrightarrow \mathbf{D}(\mathbb{Q})
$$

et nous reformulerons [5, Th. 2.57] sous une forme qui sera mieux adaptée pour la suite (cf. le Théorème 3.10). Nous introduirons ensuite deux torseurs d'isomorphismes obtenus en appliquant les foncteurs (59) aux « motifs » représentant la cohomologie de De Rham. Ces torseurs seront calculés explicitement et le lien avec l'algèbre $\mathscr{P}_{\text {For }}^{\dagger}(k)(\mathrm{cf}$. §1.3) sera expliqué. 
3.1. Les algèbres de Hopf motiviques de $k$ et $F$. — Les foncteurs (59) vérifient [4, Hypo. 1.40] avec $e=\operatorname{Sus}_{T}^{0} \circ(-)_{c s t}$ le foncteur «motif d'Artin » associé (cf. [5, Prop. 1.10] pour le cas de Bti*; le cas de $\operatorname{TgB}^{*}$ s'en déduit en utilisant la variante stable de la Remarque 2.19, mais il est tout aussi facile d'adapter l'argument utilisé pour $\mathrm{Bti}^{*}$ ). On peut donc appliquer [4, Th. 1.21 et 1.45] pour obtenir des algèbres de Hopf motiviques dans $\mathbf{D}(\mathbb{Q})$ :

$$
\mathcal{H}_{\text {mot }}(k, \sigma)=\mathrm{Bti}^{*} \mathrm{Bti}_{*} \mathbb{Q} \quad \text { et } \quad \mathcal{H}_{\text {mot }}^{\varpi}(F, \sigma)=\mathrm{TgB}^{*} \operatorname{TgB}_{*} \mathbb{Q} .
$$

On dispose également des variantes effectives :

$$
\mathcal{H}_{\text {mot }}^{\text {eff }}(k, \sigma) \quad \text { et } \quad \mathcal{H}_{\text {mot }}^{\text {eff, }}(F, \sigma)
$$

obtenues à partir des foncteurs Bti* et $\mathrm{TgB}^{*}$ sur les catégories $\mathbf{D M}^{\text {eff }}(k)$ et $\mathbf{D} \mathbf{M}^{\text {eff }}(F)$ des motifs effectifs. Ce sont seulement des bialgèbres dans $\mathbf{D}(\mathbb{Q})$, i.e., ne possédant pas d'antipodes. D'après [4, Prop. 1.48], on dispose de deux carrés commutatifs de bialgèbres
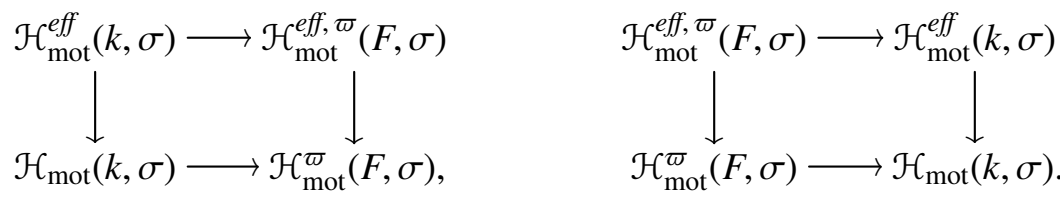

Dans le premier carré, les flèches horizontales sont déduites de $\mathrm{Bti}^{*} \simeq \operatorname{TgB}^{*} \circ(F / k)^{*}$. Dans le second carré, les flèches horizontales sont déduites de $\operatorname{TgB}^{*}=B^{*}{ }^{*} \circ \Psi_{\varpi}$. Notons aussi que les flèches horizontales dans le premier carré sont des monomorphismes scindés ayant pour rétractions respectives les flèches horizontales du second carré.

Remarque 3.1 - On fixe un plongement complexe $\sigma^{\prime}: F \hookrightarrow \mathbb{C}$ qui prolonge celui de $k$. D'après la Remarque 2.19 et sa variante stable, on a des isomorphismes (non canoniques) de bialgèbres

$$
\mathcal{H}_{\text {mot }}^{e f f, \varpi}(F, \sigma) \simeq \mathcal{H}_{\text {mot }}^{e f f}\left(F, \sigma^{\prime}\right) \quad \text { et } \quad \mathcal{H}_{\text {mot }}^{\varpi}(F, \sigma) \simeq \mathcal{H}_{\text {mot }}\left(F, \sigma^{\prime}\right) .
$$

Ces isomorphismes rendent les triangles :
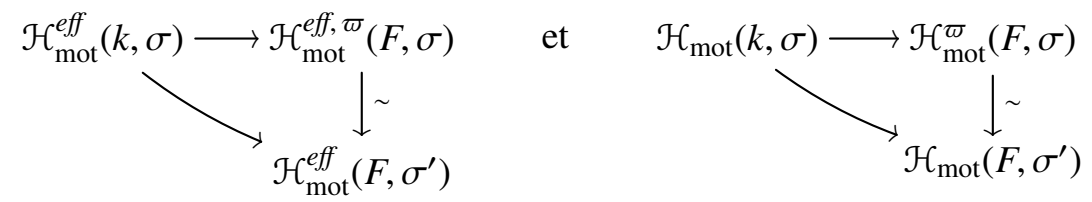

commutatifs.

Choisissons un isomorphisme Bti* $(\mathbb{Q}(1)) \simeq \mathbb{Q}$. Par adjonction, on a une flèche $\mathbb{Q}(1) \longrightarrow \mathrm{Bti}_{*} \mathbb{Q}$ dans $\mathbf{D M}^{\text {eff }}(k)$. On note $\varsigma: \mathbb{Q} \longrightarrow \mathcal{H}_{\text {mot }}^{\text {eff }}(k, \sigma)$ la composition de

$$
\mathbb{Q} \simeq \mathrm{Bti}^{*}(\mathbb{Q}(1)) \longrightarrow \mathrm{Bti}^{*}\left(\mathrm{Bti}_{*} \mathbb{Q}\right)=\mathcal{H}_{\text {mot }}^{\text {eff }}(k, \sigma) .
$$

Alors $\varsigma$ est indépendant du choix effectué (voir [4, Prop. 2.12]).

Proposition 3.2 - Notons aussi $\varsigma$ l'image de $1 \in \mathbb{Q}$ dans $\mathrm{H}_{0}\left(\mathcal{H}_{\text {mot }}^{\text {eff }}(k, \sigma)\right)$ et $\mathrm{H}_{0}\left(\mathcal{H}_{\text {mot }}^{\text {eff, } \sigma}(F, \sigma)\right)$ par les morphismes induits par $\varsigma$. On a des isomorphismes canoniques dans $\mathbf{D}(\mathbb{Q})$ :

$$
\mathcal{H}_{\text {mot }}(k, \sigma) \simeq \mathcal{H}_{\text {mot }}^{\text {eff }}(k, \sigma)\left[\varsigma^{-1}\right] \quad \text { et } \quad \mathcal{H}_{\text {mot }}^{\varpi}(F, \sigma) \simeq \mathcal{H}_{\text {mot }}^{\text {eff, } \varpi}(F, \sigma)\left[\varsigma^{-1}\right] .
$$

Demonstration Le premier isomorphisme est obtenu dans [4, Th. 2.14]. Le second isomorphisme se déduit aussi de [4, Th. 2.14] en utilisant la Remarque 3.1.

Théorème 3.3 - Les complexes $\mathcal{H}_{\text {mot }}^{\text {eff }}(k, \sigma)$ et $\mathcal{H}_{\text {mot }}(k, \sigma)$ sont $(-1)$-connexes, i.e., leur homologie est nulle en degrés strictement négatifs. Il en est de même des complexes $\mathcal{H}_{\text {mot }}^{\text {eff, }}(F, \sigma)$ et $\mathcal{H}_{\mathrm{mot}}^{\varpi}(F, \sigma)$. 
Demonstration Pour les complexes $\mathcal{H}_{\text {mot }}^{e f f}(k, \sigma)$ et $\mathcal{H}_{\text {mot }}(k, \sigma)$, il s'agit de [4, Cor. 2.105]. Pour les complexes, $\mathcal{H}_{\text {mot }}^{e f f, \varpi}(F, \sigma)$ et $\mathcal{H}_{\text {mot }}^{\varpi}(F, \sigma)$, on utilise la Remarque 3.1 pour se ramener encore à [4, Cor. 2.105].

$\mathrm{Si}$ on se restreint à la variante stable, les foncteurs $\mathrm{TgB}^{*}$ (jouant le rôle de $f_{1}$ ), Bti* (jouant le rôle de $f_{2}$ ) et $\Psi_{\varpi}$ (jouant le rôle de $k$ ) vérifient [5, Hypo. 2.6]. D'après [5, Th. 2.7], l'algèbre de Hopf $\mathcal{H}_{\text {mot }}^{\varpi}(F, \sigma)$ est naturellement le produit tensoriel semi-direct :

$$
\mathcal{H}_{\text {mot }}^{\varpi}(F, \sigma) \simeq \operatorname{Bti}^{*}\left(\mathfrak{P}^{*} \mathfrak{P}_{*} \mathbb{Q}\right) \otimes \mathcal{H}_{\text {mot }}(k, \sigma) .
$$

(Ici, on a posé $\mathfrak{P}^{*}=\Psi_{\varpi}$ et $\mathfrak{P}_{*}$ son adjoint à droite.) On obtient ainsi une suite exacte scindée d'algèbres de Hopf dans $\mathbf{D}(\mathbb{Q})$ :

$$
\mathbb{Q} \longrightarrow \mathcal{H}_{\mathrm{mot}}(k, \sigma) \longrightarrow \mathcal{H}_{\mathrm{mot}}^{\varpi}(F, \sigma) \longrightarrow \operatorname{Bti}^{*}\left(\mathfrak{P}^{*} \mathfrak{P}_{*} \mathbb{Q}\right) \longrightarrow \mathbb{Q} \text {. }
$$

Suivant [5, Déf. 2.25 et Rem. 2.26], on pose :

$$
\mathcal{H}_{/ k}^{\mathrm{rel}, \varpi}(F, \sigma)=\operatorname{Bti}^{*}\left(\mathfrak{P}^{*} \mathfrak{P}_{*} \mathbb{Q}\right) .
$$

C'est l'algèbre de Hopf motivique relative de l'extension $F / k$.

THÉORÈme 3.4 - Le complexe $\mathcal{H}_{/ k}^{\mathrm{rel}, \varpi}(F, \sigma)$ est concentré en degré zéro, i.e., son homologie est nulle en degrés différents de zéro.

Demonstration Ceci découle de [5, Th. 2.55]. On fera attention que dans loc. cit. il s'agit de l'algèbre de Hopf graduée

$$
\mathrm{H}_{\bullet}\left(\mathcal{H}_{\text {mot }}\left(F, \sigma^{\prime}\right)\right) \bigotimes_{\mathrm{H}_{\bullet}\left(\mathcal{H}_{\mathrm{mot}}(k, \sigma)\right)} \mathbb{Q} \text {. }
$$

Toutefois, cette algèbre de Hopf est isomorphe (non canoniquement) à $\mathrm{H}_{\bullet}\left(\mathcal{H}_{/ k}^{\mathrm{rel}, \varpi}(F, \sigma)\right)$ grâce à la Remarque 3.1 .

3.2. Les groupes de Galois motiviques de $k$ et $F$. - On pose :

$$
\begin{array}{cc}
\mathbf{H}_{\text {mot }}^{\text {eff }}(k, \sigma)=\mathrm{H}_{0}\left(\mathcal{H}_{\mathrm{mot}}^{\text {eff }}(k, \sigma)\right), & \mathbf{H}_{\text {mot }}^{\text {eff }}(F, \sigma)=\mathrm{H}_{0}\left(\mathcal{H}_{\text {mot }}^{\text {eff, }}(F, \sigma)\right), \\
\mathbf{H}_{\text {mot }}(k, \sigma)=\mathrm{H}_{0}\left(\mathcal{H}_{\text {mot }}(k, \sigma)\right) \quad \text { et } \quad \mathbf{H}_{\text {mot }}^{\varpi}(F, \sigma)=\mathrm{H}_{0}\left(\mathcal{H}_{\text {mot }}^{\varpi}(F, \sigma)\right) .
\end{array}
$$

Le Théorème 3.3 entraîne que les $\mathbb{Q}$-espaces vectoriels ci-dessus sont naturellement des bialgèbres; les deux derniers sont même des $\mathbb{Q}$-algèbres de Hopf. On obtient en particulier deux groupes pro-algébriques définis sur $\mathbb{Q}$ :

$$
\mathbf{G}_{\mathrm{mot}}(k, \sigma)=\operatorname{Spec}\left(\mathbf{H}_{\mathrm{mot}}(k, \sigma)\right) \quad \text { et } \quad \mathbf{G}_{\mathrm{mot}}^{\varpi}(F, \sigma)=\operatorname{Spec}\left(\mathbf{H}_{\mathrm{mot}}^{\varpi}(F, \sigma)\right) .
$$

Ce sont les groupes de Galois motiviques de $k$ et $F$ associés respectivement aux foncteurs de réalisation $\mathrm{Bti}^{*}$ et $\mathrm{TgB}^{*}$. Bien entendu, la Remarque 3.1 fournit des isomorphismes (non canoniques) entre $\mathbf{G}_{\text {mot }}^{\varpi}(F, \sigma)$ et $\mathbf{G}_{\text {mot }}\left(F, \sigma^{\prime}\right)$.

Les flèches horizontales inférieures dans les carrés (62) induisent des morphismes canoniques de groupes pro-algébriques :

$$
\mathbf{G}_{\mathrm{mot}}^{\varpi}(F, \sigma) \longrightarrow \mathbf{G}_{\mathrm{mot}}(k, \sigma) \quad \text { et } \quad \mathbf{G}_{\mathrm{mot}}(k, \sigma) \longrightarrow \mathbf{G}_{\mathrm{mot}}^{\varpi}(F, \sigma) .
$$

Le premier est une rétraction du second. Plus précisément, la décomposition en produit tensoriel semi-direct (64) fournit une décomposition en produit semi-direct

$$
\mathbf{G}_{\text {mot }}^{\varpi}(F, \sigma) \simeq \mathbf{G}_{/ k}^{\mathrm{rel}, \varpi}(F, \sigma) \rtimes \mathbf{G}_{\text {mot }}(k, \sigma)
$$

avec $\mathbf{G}_{/ k}^{\mathrm{rel}, \varpi}(F, \sigma)=\operatorname{Spec}\left(\mathrm{H}_{0}\left(\mathcal{H}_{/ k}^{\mathrm{rel}, \varpi}(F, \sigma)\right)\right)$. L'action de $\mathbf{G}_{\text {mot }}(k, \sigma)$ sur le groupe pro-algébrique $\mathbf{G}_{/ k}^{\mathrm{rel}, \varpi}(F, \sigma)$ provient du fait que l'algèbre de $\operatorname{Hopf} \mathcal{H}_{/ k}^{\mathrm{rel}, \varpi}(F, \sigma)$ est la réalisation de Betti d'une algèbre de Hopf de $\mathbf{D M}(k)$, à savoir $\mathfrak{P}^{*} \mathfrak{P}_{*} \mathbb{Q}$, et du fait que $\mathbf{G}_{\text {mot }}(k, \sigma)$ agit sur l'homologie singulière de tout objet de $\mathbf{D M}(k)$. Le groupe $\mathbf{G}_{/ k}^{\text {rel, }}(F, \sigma)$ est appelé le groupe de Galois motivique 
relatif de l'extension $F / k$. Il jouera un rôle fondamental dans la suite. Notons enfin qu'on a une suite exacte scindée de groupes pro-algébriques (cf. (65))

$$
\{1\} \longrightarrow \mathbf{G}_{/ k}^{\mathrm{rel}, \varpi}(F, \sigma) \longrightarrow \mathbf{G}_{\mathrm{mot}}^{\varpi}(F, \sigma) \longrightarrow \mathbf{G}_{\mathrm{mot}}(k, \sigma) \longrightarrow\{1\}
$$

de sorte que $\mathbf{G}_{/ k}^{\mathrm{rel}, \varpi}(F, \sigma)$ s'identifie au noyau du premier morphisme dans (68).

Remarque 3.5 - Soit $\sigma^{\prime}: F \hookrightarrow \mathbb{C}$ un plongement complexe de $F$ qui prolonge celui de $k$. Dans [5, Déf. 2.25], nous avons défini le groupe de Galois motivique relatif de l'extension $F / k$, noté $\mathbf{G}_{/ k}^{\text {rel }}\left(F, \sigma^{\prime}\right)$, comme étant le noyau du morphisme $\mathbf{G}_{\mathrm{mot}}\left(F, \sigma^{\prime}\right) \longrightarrow \mathbf{G}_{\mathrm{mot}}(k, \sigma)$. On fera attention que ce groupe n'est pas le «même » que son homonyme $\mathbf{G}_{/ k}^{\text {rel, } \varpi}(F, \sigma)$. Toutefois, il lui est isomorphe non canoniquement comme il découle de la Remarque 3.1 .

3.3. Reformulation d'un résultat de [5]. - Soit $X$ un schéma lisse. Rappelons que lisDM $(X) \subset$ $\mathbf{D M}(X)$ désigne la plus petite sous-catégorie triangulée stable par sommes infinies et contenant les $X$-motifs fortement dualisables. Pour un ouvert non vide $U \subset \mathbb{A}_{k}^{1}$, on note $\mathfrak{P}_{U}^{*}$ la composition de

$$
\operatorname{lisDM}(U) \hookrightarrow \mathbf{D M}(U) \stackrel{(F / U)^{*}}{\longrightarrow} \mathbf{D M}(F) \stackrel{\Psi_{\varpi}}{\longrightarrow} \mathbf{D M}(k) .
$$

L'adjoint à droite de $\mathfrak{P}_{U}^{*}$ sera noté $\mathfrak{P}_{U, *}$. (Avant, le foncteur de restriction au point générique était désigné par $\varpi^{*}: \mathbf{D M}(U) \longrightarrow \mathbf{D M}(F)$. À partir de maintenant, il sera plus commode de le noter $(F / U)^{*}$. Plus généralement, étant donné un morphisme de schémas $f: S^{\prime} \longrightarrow S$, on notera parfois $\left(S^{\prime} / S\right)^{*}$ au lieu de $f^{*}$.)

Le foncteur $\mathfrak{P}_{U}^{*}: \operatorname{lisDM}(U) \longrightarrow \mathbf{D M}(k)$, muni de la 2-section monoïdale $(U / k)^{*}$, satisfait à $[\mathbf{4}$, Hypo. 1.40]. Il s'ensuit que

$$
\mathcal{F}_{\text {mot }}^{\varpi}(U)=\mathfrak{P}_{U}^{*} \mathfrak{P}_{U, *} \mathbb{Q}
$$

est une algèbre de Hopf dans la catégorie $\mathbf{D M}(k)$. C'est l'algèbre de Hopf fondamentale motivique de $U$ (muni du point tangentiel $\frac{\partial}{\partial \varpi}$ en 0 ). La réalisation de Betti de $\mathcal{F}_{\text {mot }}^{\varpi}(U)$ est une algèbre de Hopf de $\mathbf{D}(\mathbb{Q})$ munie d'une coaction de $\mathcal{H}_{\text {mot }}(k, \sigma)$. De plus, en appliquant [5, Th. 2.7], on obtient un isomorphisme canonique d'algèbres de Hopf

$$
\mathrm{Bti}^{*} \mathfrak{P}_{U}^{*} \mathfrak{P}_{U, *} \mathrm{Bti}_{*} \mathbb{Q} \simeq \mathrm{Bti}^{*}\left(\mathcal{F}_{\text {mot }}^{\sigma}(U)\right) \otimes \mathcal{H}_{\text {mot }}(k, \sigma)
$$

Comme son nom l'indique, l'algèbre de Hopf fondamentale motivique $\mathcal{F}_{\text {mot }}^{\varpi}(U)$ est liée au groupe fondamental de $U^{a n}$. Expliquons comment. La sous-catégorie $\mathbf{D}_{\widehat{\mathrm{LS}}}\left(U^{a n}\right) \subset \mathbf{D}\left(U^{a n}\right)$ ayant pour objets les complexes dont l'homologie est un ind-système local admet un foncteur fibre tangentiel en $0 \in \mathbb{C}$ donné par le foncteur « cycles proches » topologique

$$
\mathfrak{P}_{U^{a n}}^{*}=\Psi_{U^{a n}}: \mathbf{D}_{\widehat{\mathrm{LS}}}\left(U^{a n}\right) \longrightarrow \mathbf{D}(\mathbb{Q}) \text {. }
$$

D'après le Lemme 2.17, on a un carré commutatif à un isomorphisme canonique près :

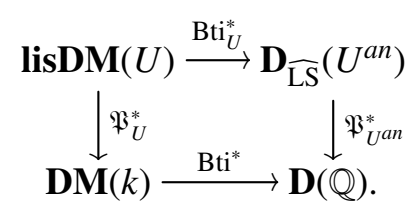

Par ailleurs, le foncteur fibre $\mathfrak{P}_{U^{a n}}^{*}: \widehat{\mathbf{L S}}\left(U^{a n}\right) \longrightarrow \operatorname{Mod}(\mathbb{Q})$ induit une équivalence de catégories :

$$
\widehat{\mathbf{L S}}\left(U^{a n}\right) \simeq \operatorname{coMod}\left(\complement_{f}^{0}\left(\pi_{1}^{\varpi}\left(U^{a n}\right), \mathbb{Q}\right)\right) .
$$

Ci-dessus, $\pi_{1}^{\varpi}\left(U^{a n}\right)$ est le groupe fondamental de $U^{a n}$ relativement au vecteur tangent $\frac{\partial}{\partial \varpi}$ en 0 et $\mathcal{C}_{f}^{0}\left(\pi_{1}^{\varpi}\left(U^{a n}\right), \mathbb{Q}\right)$ est l'algèbre de Hopf des fonctions régulières sur sa complétion pro- $\mathbb{Q}$-algébrique, i.e., la sous-algèbre des fonctions à valeurs dans $\mathbb{Q}$ sur le groupe $\pi_{1}^{\varpi}\left(U^{a n}\right)$ dont l'orbite (pour 
l'action régulière) engendre un $\mathbb{Q}$-espace vectoriel de dimension finie. En passant à l'homologie, on obtient à partir de (74) un carré commutatif à isomorphisme près :

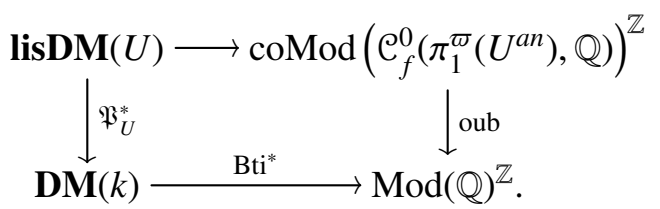

D'après [4, Prop. 1.48], on déduit un morphisme d'algèbres de Hopf

$$
\mathrm{Bti}^{*}\left(\mathcal{F}_{\text {mot }}^{\varpi}(U)\right) \longrightarrow \mathcal{C}_{f}^{0}\left(\pi_{1}^{\varpi}\left(U^{a n}\right), \mathbb{Q}\right)
$$

On peut maintenant énoncer le résultat fondamental suivant.

ThÉORÈME 3.6 - L'homologie du complexe $\mathrm{Bti}^{*}\left(\mathcal{F}_{\text {mot }}^{\sigma}(U)\right)$ est nulle en degrés différents de zéro et le morphisme (77) induit une injection sur l'homologie en degré zéro. Ainsi, l'image du morphisme

$$
\pi_{1}^{\varpi}\left(U^{a n}\right) \longrightarrow \operatorname{Spec}\left(\mathrm{H}_{0}\left(\mathrm{Bti}^{*} \mathcal{F}_{\text {mot }}^{\varpi}(U)\right)\right)
$$

est Zariski dense.

Demonstration Il s'agit d'une reformulation de [5, Th. 2.49] (voir aussi l'erratum [6]). Expliquons comment l'obtenir. Soit $u \in U(k)$ un point rationnel. Dans loc. cit., on travaille avec un autre foncteur fibre motivique, à savoir $\phi_{u}^{*}: \operatorname{lis} \mathbf{D M}(U) \longrightarrow \mathbf{D M}(k)$, obtenu en composant $u^{*}$ avec l'inclusion évidente. D'après le Théorème 2.18 , on a un isomorphisme (non canonique) $\mathrm{Bti}^{*} \circ \phi_{u}^{*} \simeq \mathrm{Bti}^{*} \circ \mathfrak{P}_{U}^{*}$ entre foncteurs de réalisation de $\operatorname{lisDM}(U)$ dans $\mathbf{D}(\mathbb{Q})$. On a aussi un isomorphisme similaire $\left(u^{a n}\right)^{*} \simeq \mathfrak{P}_{U^{a n}}^{*}$ entre foncteurs fibres de $\widehat{\mathbf{L S}}\left(U^{a n}\right)$ dans $\operatorname{Mod}(\mathbb{Q})$. De plus, ces isomorphismes sont compatibles (pour le même choix du lacet). En utilisant [4, Prop. 1.48], il s'ensuit un diagramme commutatif d'algèbres de Hopf

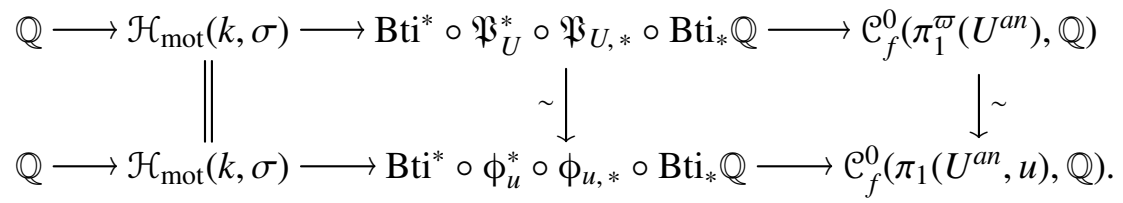

Étant donné que $\mathrm{Bti}^{*} \circ \phi_{u}^{*} \circ \phi_{u, *} \circ \mathrm{Bti}_{*} \mathbb{Q}$ est canoniquement isomorphe au produit tensoriel semidirect $\mathrm{Bti}^{*} \mathcal{F}_{\text {mot }}(U, u) \otimes \mathcal{H}_{\text {mot }}(k, \sigma)$, [5, Th. 2.49] équivaut à dire que la ligne horizontale inférieure est une suite exacte d'algèbres de Hopf, du moins après passage à l'homologie. Il en est donc de même de la ligne horizontale supérieure. On conclut maintenant à l'aide de la décomposition en produit tensoriel semi-direct (72).

Remarque 3.7 — L'argument qui nous a permis de déduire le Théorème 3.6 de [5, Th. 2.49] montre que les réalisations de Betti des algèbres de $\operatorname{Hopf} \mathcal{F}_{\text {mot }}^{\varpi}(U)$ et $\mathcal{F}_{\text {mot }}(U, u)$ sont isomorphes (non canoniquement). Nous ignorons si un tel isomorphisme est la réalisation d'un isomorphisme dans $\mathbf{D M}(k)$ entre ces algèbres de Hopf.

3.4. Application au groupe de Galois motivique relatif de $F / k_{\text {. }}$ - Pour plus de cohérence dans nos notations, posons $\mathfrak{P}^{*}=\Psi_{\varpi}: \mathbf{D M}(F) \longrightarrow \mathbf{D M}(k)$ et notons $\mathfrak{P}_{*}$ son adjoint à droite. De même que (71),

$$
\mathcal{F}_{\text {mot }}^{\varpi}(F)=\mathfrak{B}^{*} \mathfrak{P}_{*} \mathbb{Q}
$$

est une algèbre de Hopf dans la catégorie $\mathbf{D M}(k)$. C'est l'algèbre de Hopf fondamentale motivique du pro-schéma des ouverts non vides de $\mathbb{A}_{k}^{1}$. De plus, sa réalisation $\operatorname{Bti}^{*}\left(\mathcal{F}_{\text {mot }}^{\varpi}(F)\right)$ est l'algèbre de Hopf motivique relative $\mathcal{H}_{/ k}^{\text {rel, } \varpi}(F, \sigma)$ de l'extension $F / k$ (cf. (66)).

LEMME 3.8 - On a un isomorphisme canonique (aux morphismes fantômes près) dans $\mathbf{D M}(k)$ :

$$
\underset{\emptyset \neq U \subset \mathbb{A}_{k}^{1}}{\operatorname{hocolim}} \mathcal{F}_{\text {mot }}^{\varpi}(U) \simeq \mathcal{F}_{\text {mot }}^{\varpi}(F) .
$$


Demonstration Pour chaque ouvert dense $U \subset \mathbb{A}_{k}^{1}$, on a un morphisme $\mathfrak{P}_{U}^{*} \mathfrak{P}_{U, *} \mathbb{Q} \longrightarrow \mathfrak{P}^{*} \mathfrak{P}_{*} \mathbb{Q}$ déduit du triangle commutatif à isomorphisme près

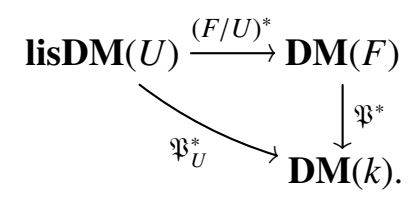

On obtient (80) en passant à la colimite homotopique suivant l'ensemble dénombrable des ouverts non vides de $\mathbb{A}_{k}^{1}$. Pour montrer que (80) est inversible, il suffit de montrer que le morphisme

$$
\underset{\emptyset \neq U \subset \mathbb{A}_{k}^{1}}{\operatorname{hocolim}}(F / U)^{*} \mathfrak{P}_{U, *} \mathbb{Q} \longrightarrow \mathfrak{P}_{*} \mathbb{Q}
$$

est inversible dans $\mathbf{D M}(F)$. Pour cela, on fixe un objet compact $M \in \mathbf{D M}(F)$ et on montre que $(81)$ induit une bijection après application de $\operatorname{hom}_{\mathbf{D M}(F)}(M,-)$. Puisque $M$ est compact, il existe un ouvert non vide $U_{0} \subset \mathbb{A}_{k}^{1}$ et $M_{0} \in \operatorname{lisDM}\left(U_{0}\right)$ tels que $M=\left(F / U_{0}\right)^{*} M_{0}$. Le morphisme

$$
\underset{\emptyset \neq V \subset U_{0} \cap U}{\operatorname{hocolim}} \operatorname{hom}_{\mathbf{D M}(V)}\left(\left.\left(M_{0}\right)\right|_{V},\left.\left(\mathfrak{P}_{U, *} \mathbb{Q}\right)\right|_{V}\right) \longrightarrow \operatorname{hom}_{\mathbf{D M}(F)}\left(M,(F / U)^{*} \mathfrak{P}_{U, *} \mathbb{Q}\right)
$$

est inversible. Il s'ensuit que (81), auquel on a appliqué $\operatorname{hom}_{\mathbf{D M}(F)}(M,-)$, s'identifie à l'homomorphisme

$$
\underset{\emptyset \neq U \subset U_{0}}{\operatorname{hocolim}} \operatorname{hom}_{\mathbf{D M}(U)}\left(\left.M_{0}\right|_{U}, \mathfrak{P}_{U, *} \mathbb{Q}\right) \longrightarrow \operatorname{hom}_{\mathbf{D M}(F)}\left(M, \mathfrak{P}_{*} \mathbb{Q}\right) \text {. }
$$

Par adjonction, cet homomorphisme se réécrit

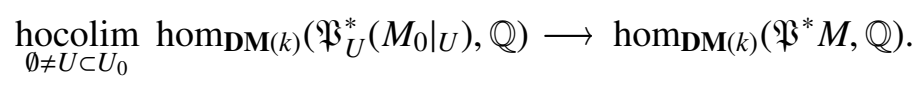

Le résultat recherché est maintenant clair.

Corollaire 3.9 - On a un isomorphisme canonique dans $\mathbf{D}(\mathbb{Q})$ :

$$
\underset{\emptyset \neq U \subset \mathbb{A}_{k}^{1}}{\operatorname{hocolim}} \operatorname{Bti}^{*}\left(\mathcal{F}_{\text {mot }}^{\varpi}(U)\right) \simeq \operatorname{Bti}^{*}\left(\mathcal{F}_{\text {mot }}^{\varpi}(F)\right)=\mathcal{H}_{/ k}^{\mathrm{rel}, \varpi}(F, \sigma)
$$

Demonstration Ceci découle du Lemme 3.8. L'isomorphisme obtenu est maintenant canonique car la réalisation de Betti du morphisme fantôme résultant de la colimite homotopique est nulle dans $\mathbf{D}(\mathbb{Q})$.

Étant donnés des ouverts non vides $V \subset U \subset \mathbb{A}_{k}^{1}$, on a un carré commutatif de schémas en groupe

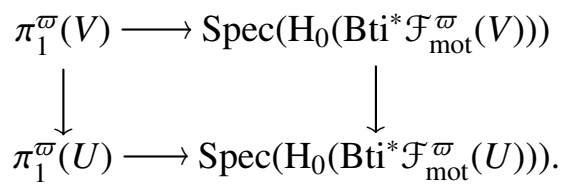

Il s'ensuit un morphisme de pro-schémas en groupe

$$
\pi_{1}^{\varpi}(\mathbb{C} \backslash \bar{k}) \longrightarrow \mathbf{G}_{/ k}^{\mathrm{rel}, \varpi}(F, \sigma) .
$$

Ci-dessus, nous avons noté $\pi_{1}^{\varpi}(C \backslash \bar{k})$ le pro-groupe $\left\{\pi_{1}^{\varpi}\left(U^{a n}\right)\right\}_{\emptyset \neq U \subset \mathbb{A}_{k}^{1}}$. C'est le pro-groupe fondamental de la pro-variété analytique $(\mathbb{C} \backslash \bar{k})=\left\{U^{a n}\right\}_{\emptyset \neq U \subset \mathbb{A}_{k}^{1}}$. Le résultat suivant jouera un rôle fondamental dans la suite.

ThÉORème 3.10 - L'image du morphisme (83) est Zariski dense. Autrement dit, le morphisme suivant est injectif:

$$
\mathrm{H}_{0}\left(\mathcal{H}_{/ k}^{\mathrm{rel}, \varpi}(F, \sigma)\right) \longrightarrow \operatorname{colim}_{\emptyset \neq U \subset \mathbb{A}_{k}^{1}} \mathcal{C}_{f}^{0}\left(\pi_{1}^{\varpi}\left(U^{a n}\right), \mathbb{Q}\right) .
$$

Demonstration Ceci s'obtient par passage à la limite à partir du Théorème 3.6 en utilisant le Corollaire 3.9. (Voir aussi [5, Th. 2.57].) 
3.5. Calculs de torseurs d'isomorphismes, première partie. - On passe maintenant aux torseurs d'isomorphismes entre réalisation de Betti et réalisation de De Rham. On rappelle d'abord les résultats de $[4, \S 2.3 .1]$.

On note $\Omega_{j k}^{\cdot}$ le complexe de préfaisceaux sur $\mathrm{Sm} / k$ qui à un $k$-schéma lisse $X$ associe le complexe $\Gamma\left(X, \Omega_{X / k}^{\circ}\right)$ des sections globales du complexe de De Rham algébrique de $X$. D'après [17], $\Omega_{\mid k}^{\cdot}$ est naturellement un complexe de préfaisceaux avec transferts, i.e., un objet de $\mathbf{D M}^{\text {eff }}(k){ }^{(1)}$ D'après [4, Cor. 2.89], il existe un isomorphisme canonique dans $\mathbf{D M}^{e f f}(k)$ :

$$
\Omega_{/ k}^{\otimes_{k}} \otimes_{k} \mathbb{C} \simeq\left(\mathrm{Bti}_{*} \mathbb{Q}\right) \otimes_{\mathbb{Q}} \mathbb{C}
$$

qui résume succinctement le théorème de comparaison de Grothendieck [13]. Il s'ensuit un isomorphisme d'algèbres dans $\mathbf{D}(\mathbb{Q})$ :

$$
\operatorname{Bti}^{*}\left(\Omega_{/ k}^{*}\right) \otimes_{k} \mathbb{C} \simeq \mathcal{H}_{\text {mot }}^{e f f}(k, \sigma) \otimes_{\mathbb{Q}} \mathbb{C} .
$$

On est naturellement amené à poser :

$$
\mathcal{P}^{e f f}(k, \sigma)=\operatorname{Bti}^{*}\left(\Omega_{/ k}^{\bullet}\right)
$$

Par définition $\mathcal{P}^{\text {eff }}(k, \sigma)$ est un comodule sur la bialgèbre $\mathcal{H}_{\text {mot }}^{\text {eff }}(k, \sigma)$ (cf. [4, Prop. 1.28]). On a le résultat suivant qui découle de [4, Th. 2.104].

ThÉorème 3.11 - Le complexe $\operatorname{Peff}^{(k, \sigma)}$ est $(-1)$-connexe, i.e., son homologie est nulle en degrés strictement négatifs.

On pose :

$$
\mathbf{P}^{e f f}(k, \sigma)=\mathrm{H}_{0}\left(\mathcal{P}^{e f f}(k, \sigma)\right) .
$$

C'est l'anneau des périodes formelles effectives qui est naturellement un comodule sur $\mathbf{H}^{\text {eff }}(k, \sigma)$. En fait, le pro-schéma $\operatorname{Spec}\left(\mathbf{P}^{e f f}(k, \sigma)\right)$ est un espace homogène sous l'action du pro-schéma en monoïde $\operatorname{Spec}\left(\mathbf{H}^{e f f}(k, \sigma)\right)$. (Il s'agit d'une conséquence immédiate de l'isomorphisme (85).)

On a également la variante stable. Pour cela il est plus pratique de prendre comme représentant du motif de Tate le préfaisceau avec transferts $T=\mathbb{Q}_{t r}(U, u)$ où $U=\mathbb{P}_{k}^{1} \times_{k} \mathbb{P}_{k} \backslash \Delta\left(\mathbb{P}_{k}^{1}\right)$ et $u=(\infty, 0)$. Ceci est possible puisque la projection sur le premier facteur $U \longrightarrow \mathbb{P}_{k}^{1}$ est un torseur sous $\mathbf{G}_{\boldsymbol{a}}$ et induit donc une équivalence ( $\mathbb{A}^{1}$, ét)-locale. Le point est que $U$ est affine de sorte que son deuxième groupe de cohomologie de De Rham algébrique est engendré par la classe d'une forme différentielle $v \in \Omega_{/ k}^{2}(U)$. On note $\boldsymbol{\Omega}_{/ k}$ le $T$-spectre (non symétrique) donné en niveau $n$ par $\Omega_{/ k}[2 n]$. Les morphismes d'assemblage correspondent par adjonction aux morphismes

$$
\Omega_{/ k}^{\bullet}[2 n] \longrightarrow \underline{\operatorname{hom}}\left((U, u), \Omega_{/ k}^{\bullet}[2]\right)[2 n]
$$

qui associent à $\omega \in \Omega_{/ k}^{\bullet}(X)$ la forme différentielle $v \otimes \omega \in \Omega_{/ k}^{2+\bullet}\left((U, u) \times_{k} X\right)$. D'après [4, Prop. 2.92], il existe un isomorphisme canonique dans $\mathbf{D M}(k)$ :

$$
\mathbf{\Omega}_{/ k} \otimes_{k} \mathbb{C} \simeq\left(\mathrm{Bti}_{*} \mathbb{Q}\right) \otimes_{\mathbb{Q}} \mathbb{C} .
$$

Il s'ensuit un isomorphisme d'algèbres dans $\mathbf{D}(\mathbb{Q})$ :

$$
\operatorname{Bti}^{*}\left(\boldsymbol{\Omega}_{/ k}\right) \otimes_{k} \mathbb{C} \simeq \mathcal{H}_{\text {mot }}(k, \sigma) \otimes_{\mathbb{Q}} \mathbb{C} .
$$

On est naturellement amené à poser :

$$
\mathcal{P}(k, \sigma)=\mathrm{Bti}^{*}\left(\mathbf{\Omega}_{/ k}\right) .
$$

Par définition $\mathcal{P}(k, \sigma)$ est un comodule sur l'algèbre de Hopf $\mathcal{H}_{\text {mot }}(k, \sigma)$ (cf. [4, Prop. 1.28]).

1. Strictement parlant, il n'est pas nécessaire de munir $\Omega_{/ k}^{\bullet}$ de transferts. En effet, comme dans $[\mathbf{4}, \S 2.3 .1]$, on peut travailler avec DA ${ }^{e f f}(k)$ au lieu de $\mathbf{D} \mathbf{M}^{e f f}(k)$. De même, la Remarque 2.40 montre qu'il n'y a pas besoin de munir $\Omega_{/ F}^{*}$ de transferts dans le paragraphe suivant. 
Le complexe $\mathcal{P}(k, \sigma)$ est une localisation de son analogue effectif $\mathcal{P}^{e f f}(k, \sigma)$. En effet, soit $\bar{\pi} \in$ $\mathrm{H}_{0}\left(\mathcal{P}^{\text {eff }}(k, \sigma)\right)$ l'image de $\alpha \in \mathrm{H}_{2}\left(\mathrm{Bti}^{*} \mathbb{Q}_{t r}\left(\mathbb{P}_{k}^{1}, \infty\right)\right)$ par la composition de

$$
\mathrm{Bti}^{*} \mathbb{Q}_{t r}\left(\mathbb{P}_{k}^{1}, \infty\right)[-2] \simeq \mathrm{Bti}^{*} \mathbb{Q}_{t r}(U, u)[-2] \stackrel{v}{\longrightarrow} \operatorname{Bti}^{*}\left(\Omega_{/ k}^{*}\right) .
$$

On a le résultat suivant (cf. [4, Th. 2.93]).

LEMME 3.12 - On a un isomorphisme canonique :

$$
\mathcal{P}(k, \sigma) \simeq \mathcal{P}^{e f f}(k, \sigma)\left[\bar{\pi}^{-1}\right] .
$$

Vu le Lemme 3.12, il découle du Théorème 3.11 que le complexe $\mathcal{P}(k, \sigma)$ est aussi $(-1)$ connexe. On pose :

$$
\mathbf{P}(k, \sigma)=\mathrm{H}_{0}(\mathcal{P}(k, \sigma))
$$

C'est l'anneau des périodes formelles qui est naturellement un comodule sur $\mathbf{H}(k, \sigma)$. En fait, le pro-schéma $\operatorname{Spec}(\mathbf{P}(k, \sigma))$ est un $k$-torseur sous le groupe de Galois motivique $\mathbf{G}_{\mathrm{mot}}(k, \sigma)$. (Ceci découle immédiatement de l'isomorphisme (90).)

Nous allons maintenant expliquer comment «calculer » le complexe $\mathcal{P}^{e f f}(k, \sigma)$. En utilisant le Théorème 2.21, on trouve un isomorphisme canonique :

$$
\operatorname{Sg}_{\text {ét }}^{\mathbb{D}}\left(\Omega_{/ k}^{\cdot}\right) \simeq \operatorname{Bti}^{*}\left(\Omega_{\mid k}^{\cdot}\right)=\mathcal{P}^{e f f}(k, \sigma) .
$$

Le complexe $\operatorname{Sg}_{\text {ét }}^{\mathbb{D}}\left(\Omega_{j k}^{\bullet}\right)$ est un cas particulier du complexe de De Rham associé à un $\mathrm{W}(k)$-module cubique $\left(\Sigma\right.$-enrichi) au sens de [4, Déf. 2.94]. Le W $(k)$-module cubique qu'on prend est $\mathcal{O}\left(\overline{\mathbb{D}}_{\dot{e} \mathrm{t}}\right)=$ $\mathcal{O}_{k-a l g}\left(\overline{\mathbb{D}}^{\bullet}\right)$ (voir le Lemme 2.22). En appliquant [4, Prop. 2.100], on déduit alors un isomorphisme canonique dans $\mathbf{D}(\mathbb{Q})$ :

$$
\operatorname{Sg}_{\text {ét }}^{\mathbb{D}}\left(\Omega_{\mid k}^{\bullet}\right) \simeq \widetilde{\Omega}^{\infty-\bullet}\left(\mathcal{O}\left(\overline{\mathbb{D}}_{\text {ét }}\right)\right) .
$$

Ci-dessus, on a

$$
\widetilde{\Omega}^{\infty-n}\left(\mathcal{O}\left(\overline{\mathbb{D}}_{\mathrm{é}}\right)\right)=\bigoplus_{I \subset \mathbb{N} \backslash\{0\}, \operatorname{card}(I)=n} \mathcal{O}_{k-\text { alg }}^{(I)}\left(\overline{\mathbb{D}}^{\infty}\right) \cdot \mathrm{d} \hat{z}_{I}
$$

où $\mathcal{O}_{k-\text { alg }}^{(I)}\left(\overline{\mathbb{D}}^{\infty}\right)$ est le sous- $k$-espace vectoriel de $\mathcal{O}_{k \text {-alg }}\left(\overline{\mathbb{D}}^{\infty}\right)$ formé des fonctions $f$ tels que $\left.f\right|_{z_{i}=\epsilon}=$ 0 pour $i \in I$ et $\epsilon \in\{0,1\}$. Bien entendu, $\mathrm{d} \hat{z}_{I}$ est la forme différentielle de degré infini obtenue en prenant le produit extérieur de tous les $\mathrm{d} z_{i}$ pour $i \notin I$ et la différentielle de $\widetilde{\Omega}^{\infty-\bullet}\left(\mathcal{O}\left(\overline{\mathbb{D}}_{\text {ét }}\right)\right)$ est celle de De Rham. En passant à l'homologie en degré zéro dans (95), on obtient le résultat suivant (cf. [4, Prop. 2.108]).

ThÉorème 3.13 - Il existe un isomorphisme canonique entre $\mathbf{P}^{\text {eff }}(k, \sigma)$ et le quotient du $k$-espace vectoriel $\mathcal{O}_{k-a l g}\left(\overline{\mathbb{D}}^{\infty}\right)$ par son sous-espace engendré par les éléments de la forme

$$
\frac{\partial g}{\partial z_{i}}-\left.g\right|_{z_{i}=1}+\left.g\right|_{z_{i}=0}
$$

avec $i \in \mathbb{N} \backslash\{0\}$ et $g \in \mathcal{O}_{k-a l g}\left(\overline{\mathbb{D}}^{\infty}\right)$. De plus, $\mathbf{P}(k, \sigma)=\mathbf{P}^{\text {eff }}(k, \sigma)\left[\bar{\pi}^{-1}\right]$.

3.6. Calculs de torseurs d'isomorphismes, deuxième partie. - Notre but ici est d'introduire et de calculer l'algèbre du torseur d'isomorphismes entre la réalisation de Betti tangentielle et la réalisation de De Rham.

On fixe un plongement complexe $\sigma^{\prime}: F \hookrightarrow \mathbb{C}$ qui prolonge celui de $k$. Les notations et les constructions du paragraphe précédent s'appliquent à $F$ et $\sigma^{\prime}$. Vu la Remarque 2.19, les isomorphismes (85) et (89) induisent des isomorphismes (non canoniques cette fois !) dans $\mathbf{D} \mathbf{M}^{\text {eff }}(F)$ et $\mathbf{D M}(F)$ respectivement :

$$
\Omega_{j F}^{\cdot} \otimes_{F} \mathbb{C} \simeq\left(\mathrm{TgB}_{*} \mathbb{Q}\right) \otimes_{\mathbb{Q}} \mathbb{C} \quad \text { et } \quad \boldsymbol{\Omega}_{/ F} \otimes_{F} \mathbb{C} \simeq\left(\operatorname{TgB}_{*} \mathbb{Q}\right) \otimes_{\mathbb{Q}} \mathbb{C} .
$$

En appliquant $\operatorname{TgB}^{*}$ à ces isomorphismes, on déduit des isomorphismes dans $\mathbf{D}(\mathbb{Q})$ :

$$
\operatorname{TgB}^{*}\left(\Omega_{/ F}^{\cdot}\right) \otimes_{F} \mathbb{C} \simeq \mathcal{H}_{\text {mot }}^{e f f, \varpi}(F, \sigma) \otimes_{\mathbb{Q}} \mathbb{C} \quad \text { et } \quad \operatorname{TgB}^{*}\left(\boldsymbol{\Omega}_{/ F}\right) \otimes_{F} \mathbb{C} \simeq \mathcal{H}_{\text {mot }}^{\varpi}(F, \sigma) \otimes_{\mathbb{Q}} \mathbb{C} .
$$


On pose :

$$
\mathcal{P}^{e f f}, \varpi(F, \sigma)=\operatorname{TgB}^{*}\left(\Omega_{/ F}^{*}\right) \quad \text { et } \quad \mathcal{P}^{\varpi}(F, \sigma)=\operatorname{TgB}^{*}\left(\mathbf{\Omega}_{/ F}\right) .
$$

Ce sont naturellement des comodules sous les bialgèbres motiviques $\mathcal{H}_{\text {mot }}^{\text {eff }}$ ( $(F, \sigma)$ et $\mathcal{H}_{\text {mot }}^{\varpi}(F, \sigma)$ (cf. [4, Prop. 1.28]). Le résultat suivant découle de [4, Th. 2.104] et de la Remarque 2.19.

ThÉoRÈme 3.14 - Les complexes $\mathcal{P}^{\text {eff, }}{ }^{\varpi}(F, \sigma)$ et $\mathcal{P}^{\varpi}(F, \sigma)$ sont $(-1)$-connexes, i.e., leur homologie est nulle en degrés strictement négatifs.

On pose :

$$
\mathbf{P}^{e f f, \varpi}(F, \sigma)=\mathrm{H}_{0}\left(\mathcal{P}^{e f f}, \varpi(F, \sigma)\right) \quad \text { et } \quad \mathbf{P}^{\varpi}(F, \sigma)=\mathrm{H}_{0}\left(\mathcal{P}^{\varpi}(F, \sigma)\right) .
$$

Ce sont naturellement des comodules sous les bialgèbres motiviques $\mathbf{H}_{\text {mot }}^{\text {eff }}(F, \sigma)$ et $\mathbf{H}_{\text {mot }}^{\varpi}(F, \sigma)$. En fait, $\operatorname{Spec}\left(\mathbf{P}^{e f f, \varpi}(F, \sigma)\right)$ est un espace homogène sous le schéma en monoïde $\operatorname{Spec}\left(\mathbf{H}_{\operatorname{mot}}^{\text {eff, }}(F, \sigma)\right)$. De même, $\operatorname{Spec}\left(\mathbf{P}^{\varpi}(F, \sigma)\right)$ est un $F$-torseur sous le groupe de Galois motivique $\mathbf{G}_{\text {mot }}^{\varpi}(F, \sigma)$. Il s'agit d'une conséquence immédiate des isomorphismes (97).

Proposition 3.15 - Il existe des carrés commutatifs
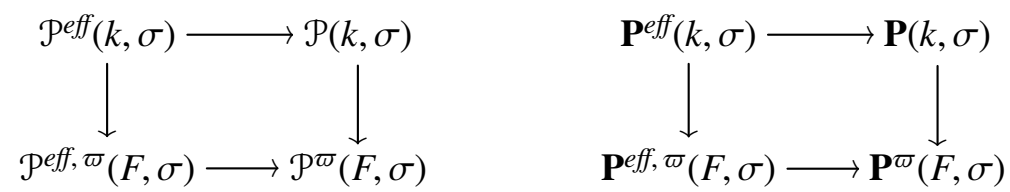

où les morphismes sont compatibles aux coactions des bialgèbres motiviques. En particulier, le morphisme

$$
\operatorname{Spec}\left(\mathbf{P}^{\varpi}(F, \sigma)\right) \longrightarrow \operatorname{Spec}(\mathbf{P}(k, \sigma))
$$

est $\mathbf{G}_{\text {mot }}^{\sigma}(F, \sigma)$-équivariant. (Bien entendu, l'action de $\mathbf{G}_{\text {mot }}^{\sigma}(F, \sigma)$ sur $\operatorname{Spec}(\mathbf{P}(k, \sigma))$ est la restriction de l'action de $\mathbf{G}_{\mathrm{mot}}(k, \sigma)$ suivant le premier morphisme de (68).)

Demonstration C'est une application directe de [4, Prop. 1.48].

Dans la suite de ce paragraphe, nous allons calculer le complexe $\mathcal{P}^{\varpi}(F, \sigma)$. Notre outil principal sera le Théorème 2.39. Précisons d'abord que dans la définition de $\boldsymbol{\Omega}_{/ F}$ nous prendrons pour $v \in \Omega_{/ F}^{2}\left(U_{F}\right)$ l'image de $v \in \Omega_{/ k}^{2}(U)$ par le morphisme de changement de base. (Ici nous avons noté $U_{F}=U \otimes_{k} F$.) Ceci permet d'écrire plus facilement le morphisme canonique $(F / k)^{*} \boldsymbol{\Omega}_{/ k} \longrightarrow \boldsymbol{\Omega}_{/ F}$. On choisit également $\alpha^{\prime} \in \operatorname{Cor}_{k}\left(\overline{\mathbb{D}}_{\text {éte }}^{2}, U\right)$ un cycle dans le complexe $\mathrm{C}\left(\operatorname{Cor}_{k}\left(\overline{\mathbb{D}}_{\text {ét }}, U\right)\right)$ dont la classe d'homologie est envoyée sur celle de $\alpha \in \operatorname{Cor}_{k}\left(\overline{\mathbb{D}}_{\text {ét }}^{2}, \mathbb{P}_{k}^{1}\right)$. On notera de même $\alpha^{\prime} \in \operatorname{Cor}_{F}\left(\overline{\mathbb{D}}_{\text {êt }}^{\dagger, 2}, U_{F}\right)$ la correspondance finie déduite de $\alpha^{\prime}$.

Proposition 3.16 - Il existe un isomorphisme canonique dans $\mathbf{D}(\mathbb{Q})$ :

$$
\mathcal{P}^{\varpi}(F, \sigma) \simeq \underset{n \in \mathbb{N}}{\operatorname{colim}} \operatorname{colim}_{r \in \mathbb{N}^{x}} \operatorname{Tot}\left(\mathrm{C}\left(\Omega_{j F}^{\bullet}\left(\overline{\mathbb{D}}_{\text {êt }}^{\dagger} \times_{F} \mathscr{R}_{\varpi}(-, r)\right)\right)\right) .
$$

Le morphisme de transition en $n \in \mathbb{N}$ est induit par la composition de :

$$
\Omega_{/ F}^{d}\left(\overline{\mathbb{D}}_{\mathrm{e} t}^{\dagger, m} \times_{F} \mathscr{R}_{\varpi}\right) \stackrel{\nu \otimes-}{\longrightarrow} \Omega_{/ F}^{2+d}\left((U, u) \times_{F} \overline{\mathbb{D}}_{\mathrm{e} t}^{\dagger, m} \times_{F} \mathscr{R}_{\varpi}\right) \stackrel{\alpha^{\prime *}}{\longrightarrow} \Omega_{/ F}^{2+d}\left(\overline{\mathbb{D}}_{\mathrm{e} t}^{\dagger, 2+m} \times_{F} \mathscr{R}_{\varpi}\right) .
$$

Demonstration Il s'agit du Théorème 2.39 appliqué au $T$-spectre $\boldsymbol{\Omega}_{/ F}$.

On pose

$$
\text { 'Peff, } \varpi(F, \sigma)=\operatorname{colim}_{r \in \mathbb{N}^{x}} \operatorname{Tot}\left(\mathrm{C}\left(\Omega_{/ F}^{\cdot}\left(\overline{\mathbb{D}}_{\text {ét }}^{\dagger} \times_{F} \mathscr{R}_{\varpi}(-, r)\right)\right)\right) .
$$

Puisque $\mathcal{P}^{e f f}(k, \sigma)$ est canoniquement isomorphe à $\operatorname{Sg}_{\text {ét }}^{\mathbb{D}}\left(\Omega_{\mid k}^{\bullet}\right)=\operatorname{Tot}\left(\mathrm{C}\left(\Omega_{\mid k}^{\bullet}\left(\overline{\mathbb{D}}_{\text {ét }}\right)\right)\right)$, on dispose d'un morphisme canonique

$$
\mathcal{P}^{e f f}(k, \sigma) \longrightarrow \text { 'Peff, } \varpi^{(F, \sigma)} \text {. }
$$

Par construction, 'Peff, $\varpi(F, \sigma)$ est une $F$-algèbre dans $\mathbf{D}(F)$ et (101) est un morphisme de $k$ algèbres. Comme dans le Lemme 3.12, on a un isomorphisme canonique

$$
\mathcal{P}^{\varpi}(F, \sigma) \simeq \mathfrak{P}^{e f f}, \varpi(F, \sigma)\left[\bar{\pi}^{-1}\right]
$$


où, par abus de notation, $\bar{\pi} \in \mathrm{H}_{0}$ ('Peff, $\left.\varpi(F, \sigma)\right)$ désigne l'image de $\bar{\pi} \in \mathrm{H}_{0}\left(\mathcal{P}^{e f f}(k, \sigma)\right)$ par (101). Il est donc suffisant de calculer le complexe 'Peff, $\varpi(F, \sigma)$.

Remarque 3.17 — Nous tenons à avertir le lecteur que nous ne prétendons pas que 'Peff, $\varpi(F, \sigma)$ coïncide avec le complexe $\mathcal{P}^{e f f}, \varpi(F, \sigma)$. En fait, il est facile de construire un morphisme canonique 'Peff, $\varpi(F, \sigma) \longrightarrow \mathcal{P}^{e f f}$, $\varpi(F, \sigma)$, mais nous ignorons si ce morphisme est inversible. Pour la suite, on se contentera de noter qu'on a un carré commutatif :

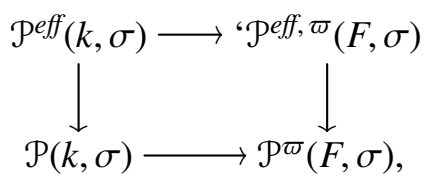

où les flèches verticales sont des morphismes de localisation par rapport à $\bar{\pi}$. Ceci découle aussitôt de la discussion précédente.

Lemme 3.18 - Pour $r \in \mathbb{N}^{\times}$, on a un isomorphisme canonique de complexes de préfaisceaux avec transferts :

$$
\operatorname{Tot}\left(\mathrm{C}\left(\underline{\operatorname{hom}}\left(\mathscr{R}_{\varpi}(-, r), \Omega_{/ F}^{*}\right)\right)\right) \simeq \Omega_{/ F}^{\cdot} \otimes_{F} F\left(\varpi^{1 / r}\right)[\ln \varpi]
$$

où $\ln \varpi$ désigne une variable transcendante sur $F$.

Demonstration Par la formule de Künneth pour la cohomologie de De Rham algébrique, il suffit de construire un isomorphisme canonique dans $\mathbf{D}(\mathbb{Q})$ :

$$
\operatorname{Tot}\left(\mathrm{N}\left(\Omega_{/ F}^{\cdot}\left(\mathscr{R}_{\varpi}(-, r)\right)\right)\right) \simeq F\left(\varpi^{1 / r}\right)[\ln \varpi]
$$

avec $\mathrm{N}$ le foncteur « complexe normalisé associé ». Rappelons que $\mathrm{N}_{n}\left(\Omega_{j F}^{\cdot}\left(\mathscr{R}_{\varpi}(-, r)\right)\right)$ est le conoyau du morphisme

$$
\bigoplus_{\underline{\mathbf{n}} \rightarrow \underline{\mathbf{n}-\mathbf{1}}} \Omega_{j F}^{\cdot}\left(F\left(\varpi^{1 / r}\right) \otimes_{F}\left(\mathbf{G}_{\boldsymbol{m} F}\right)^{n-1}\right) \longrightarrow \Omega_{/ F}^{\cdot}\left(F\left(\varpi^{1 / r}\right) \otimes_{F}\left(\mathbf{G}_{\boldsymbol{m} F}\right)^{n}\right)
$$

où les morphismes sont induits par les $n$ différentes projections de $\left(\mathbf{G}_{\boldsymbol{m} F}\right)^{n} \operatorname{sur}\left(\mathbf{G}_{\boldsymbol{m} F}\right)^{n-1}$. Il en découle que $\mathrm{N}_{n}\left(\Omega_{/ F}^{\bullet}\left(\mathscr{R}_{\varpi}(-, r)\right)\right)$ est acyclique sauf en degré $n$ où la cohomologie est un $F\left(\varpi^{1 / r}\right)$ espace vectoriel de rang 1 engendré par la classe de la forme différentielle $\frac{\mathrm{d} t_{1}}{t_{1}} \wedge \cdots \wedge \frac{\mathrm{d} t_{n}}{t_{n}}$.

Pour $n \in \mathbb{N}$, on dispose d'un morphisme canonique

$$
\int_{1}^{\varpi^{1 / r}}: \Omega_{/ F}^{n}\left(\mathscr{R}_{\varpi}(\underline{\mathbf{n}}, r)\right)=\Omega_{/ F}^{n}\left(\operatorname{Spec}\left(F\left(\varpi^{1 / r}\right)\left[t_{1}, t_{1}^{-1}, \cdots, t_{n}, t_{n}^{-1}\right]\right)\right) \longrightarrow F\left(\varpi^{1 / r}\right)[\ln \varpi]
$$

qui envoie $f \cdot \mathrm{d} t_{1} \wedge \cdots \wedge \mathrm{d} t_{n}$ sur $\int_{1}^{\varpi^{1 / r}} f \cdot \mathrm{d} t_{1} \cdots \mathrm{d} t_{n}$. (On utilise ici la relation $\ln \left(\varpi^{1 / r}\right)=r^{-1} \ln \varpi$.) Ce morphisme induit un quasi-isomorphisme

$$
\mathrm{N}_{n}\left(\Omega_{j F}^{\cdot}\left(\mathscr{R}_{\varpi}(-, r)\right)\right) \longrightarrow F\left(\varpi^{1 / r}\right) \cdot(\ln \varpi)^{n}[-n]
$$

où le membre de droite est le $F\left(\varpi^{1 / r}\right)$-espace vectoriel de dimension 1 engendré par $(\ln \varpi)^{n}$ et placé en degré cohomologique $n$. En faisant varier $n$, on obtient en fait un morphisme de bicomplexes

$$
\mathrm{N}\left(\Omega_{/ F}^{\bullet}\left(\mathscr{R}_{\varpi}(-, r)\right)\right) \longrightarrow \bigoplus_{n \in \mathbb{N}} F\left(\varpi^{1 / r}\right) \cdot(\ln \varpi)^{n}[(-n, n)]
$$

où le bicomplexe à droite à toutes ses différentielles nulles. En passant aux complexes totaux associés, on obtient le résultat recherché.

Corollaire 3.19 - On a un isomorphisme canonique de complexes de préfaisceaux avec transferts :

$$
\underset{r \in \mathbb{N}^{\times}}{\operatorname{colim}} \operatorname{Tot}\left(\mathrm{C}\left(\underline{\operatorname{hom}}\left(\mathscr{R}_{\varpi}(-, r), \Omega_{/ F}^{\cdot}\right)\right)\right) \simeq \Omega_{/ F}^{\cdot} \otimes_{F} \widehat{F}[\ln \varpi]
$$

où $\widehat{F}=F\left(\varpi^{1 / r} ; r \in \mathbb{N}^{\times}\right)$. 
Proposition 3.20 - Il existe un isomorphisme canonique dans $\mathbf{D}(\mathbb{Q})$ :

$$
\mathcal{P}^{\varpi}(F, \sigma) \simeq\left(\operatorname{colim}_{n \in \mathbb{N}} \operatorname{Tot}\left(C\left(\Omega_{/ F}^{*}\left(\overline{\mathbb{D}}_{\text {ét }}^{\dagger}\right)\right)\right) \otimes_{F} \widehat{F}[\ln \varpi] .\right.
$$

Le morphisme de transition en $n \in \mathbb{N}$ est induit par la composition de :

$$
\Omega_{/ F}^{d}\left(\overline{\mathbb{D}}_{\mathrm{e} t}^{\dagger, m}\right) \stackrel{\nu \otimes-}{\longrightarrow} \Omega_{/ F}^{2+d}\left((U, u) \times_{F} \overline{\mathbb{D}}_{\mathrm{e} t}^{\dagger, m}\right) \stackrel{\alpha^{\prime *}}{\longrightarrow} \Omega_{/ F}^{2+d}\left(\overline{\mathbb{D}}_{\mathrm{e} t}^{\dagger, 2+m}\right) .
$$

Le reste du calcul de 'Peff, $\varpi(F, \sigma)$ est similaire à celui du calcul de $\mathcal{P}^{e f f}(k, \sigma)$. En effet, c'est encore un cas particulier de [4, Prop. 2.100] appliqué au W( $k$-module cubique $\left(\Sigma\right.$-enrichi) $\mathcal{O}\left(\overline{\mathbb{D}}_{\text {ét }}^{\dagger}\right)=$ $\mathcal{O}_{k-a l g}^{\dagger}\left(\overline{\mathbb{D}}^{\bullet}\right)$ (voir le Lemme 2.28). On a donc le résultat suivant.

THÉORÈme 3.21 - Il existe un isomorphisme canonique

$$
\cdot \mathcal{P} e f f, \varpi(F, \sigma) \simeq Q^{e f f, \varpi}(F, \sigma) \otimes_{F} \widehat{F}[\ln \varpi]
$$

où $Q^{e f f, \varpi}(F, \sigma)=\widetilde{\Omega}_{k-a l g}^{\dagger, \infty-\bullet}\left(\overline{\mathbb{D}}^{\infty}\right)$ est le complexe placé en degrés homologiques positifs et défini de manière suivante. Pour $d \in \mathbb{N}$, on a

$$
\widetilde{\Omega}_{k-a l g}^{\dagger, \infty-d}\left(\overline{\mathbb{D}}^{\infty}\right)=\bigoplus_{I \subset \mathbb{N} \backslash\{0\}, \operatorname{card}(I)=d} \mathcal{O}_{k-a l g}^{\dagger,(I)}\left(\overline{\mathbb{D}}^{\infty}\right) \cdot \mathrm{d} \hat{z}_{I}
$$

où $\mathcal{O}_{k-a l g}^{\dagger,(I)}\left(\overline{\mathbb{D}}^{\infty}\right)$ est le sous-F-espace vectoriel de $\mathcal{O}_{k \text {-alg }}^{\dagger}\left(\overline{\mathbb{D}}^{\infty}\right)$ formé des séries de Laurent $G$ telles que $\left.G\right|_{z_{i}=\epsilon}=0$ pour $i \in I$ et $\epsilon \in\{0,1\}$. Le symbole $\mathrm{d} \hat{z}_{I}$ désigne le produit extérieur des formes différentielles $\mathrm{d} z_{j}$ pour $j \notin I$. Enfin, la différentielle de ce complexe est celle de De Rham.

Pour une utilisation future, on note le résultat suivant.

Proposition 3.22 - Le complexe $Q^{\text {eff, }}{ }^{(}(F, \sigma)$ est canoniquement isomorphe à $\mathrm{Bti}^{*} \chi\left(\Omega_{j F}^{\bullet}\right)$ avec $\chi$ le foncteur introduit dans le Théorème 2.24 .

Demonstration Ceci est une conséquence du Corollaire 2.27 et de [4, Prop. 2.100].

On pose

$$
\text { 'P } \mathbf{P}^{e f f}, \varpi(F, \sigma)=\mathrm{H}_{0}\left({ }^{\prime}{ }^{e f f}, \varpi(F, \sigma)\right) \quad \text { et } \quad \mathbf{Q}^{e f f}, \varpi(F, \sigma)=\mathrm{H}_{0}\left(Q^{e f f}, \varpi(F, \sigma)\right) .
$$

Un calcul similaire à celui de [4, Prop. 2.108] donne alors le résultat suivant.

Corollaire 3.23 - Le F-espace vectoriel $\mathbf{Q}^{\text {eff, }}(F, \sigma)$ est le quotient de $\mathcal{O}_{k \text {-alg }}^{\dagger}\left(\overline{\mathbb{D}}^{\infty}\right)$ par le sous-espace engendré par les éléments de la forme

$$
\frac{\partial G}{\partial z_{i}}-\left.G\right|_{z_{i}=1}+\left.G\right|_{z_{i}=0}
$$

avec $G \in \mathcal{O}_{k-a l g}^{\dagger}\left(\overline{\mathbb{D}}^{\infty}\right)$ et $i \in \mathbb{N} \backslash\{0\}$. De plus, on a un isomorphisme canonique :

$$
\cdot \mathbf{P}^{e f f, \varpi}(F, \sigma) \simeq \mathbf{Q}^{e f f, \varpi}(F, \sigma) \otimes_{F} \widehat{F}[\ln \varpi] .
$$

Remarque 3.24 - Les $F$-espaces vectoriels $\mathbf{Q}^{e f f, \varpi}(F, \sigma)$ et ' $\mathbf{P}^{e f f}{ }^{\text {f }}(F, \sigma)$ sont en fait des $F$ algèbres. La multiplication dans $\mathbf{Q}^{e f f, \varpi}(F, \sigma)$ est décrite comme dans [4, Prop. 2.108, (b)]. Le morphisme canonique $\mathbf{P}^{e f f}(k, \sigma) \longrightarrow{ }^{e f f}$, $(F, \sigma)$ se factorise $\operatorname{par} \mathbf{Q}^{e f f, \varpi}(F, \sigma)$ induisant un morphisme de $k$-algèbres

$$
\mathbf{P}^{e f f}(k, \sigma) \longrightarrow \mathbf{Q}^{e f f, \varpi}(F, \sigma) .
$$

Il est facile de voir que ce morphisme correspond, via les isomorphismes du Théorème 3.13 et du Corollaire 3.23, au morphisme induit par l'inclusion $\mathcal{O}_{k-a l g}\left(\overline{\mathbb{D}}^{\infty}\right) \hookrightarrow \mathcal{O}_{k-a l g}^{\dagger}\left(\overline{\mathbb{D}}^{\infty}\right)$. 
3.7. Lien avec les $k$-séries de périodes. - L'élément $\bar{\pi} \in \mathbf{P}^{e f f}$, $\varpi(F, \sigma)$ appartient à la sousalgèbre $\mathbf{Q}^{e f f, \varpi}(F, \sigma)$. On peut donc poser $\mathbf{Q}^{\varpi}(F, \sigma)=\mathbf{Q}^{e f f, \varpi}(F, \sigma)\left[\bar{\pi}^{-1}\right]$. C'est une sous- $F$-algèbre de $\mathbf{P}^{\varpi}(F, \sigma)$. La variante stable de la Proposition 3.22 est également vraie, i.e., $\mathbf{Q}^{\varpi}(F, \sigma)$ est canoniquement isomorphe à $\mathrm{Bti}^{*} \chi\left(\mathbf{\Omega}_{\mid F}\right)$. D'après la Remarque 3.24, on a un diagramme commutatif de $k$-algèbres à carrés cocartésiens :

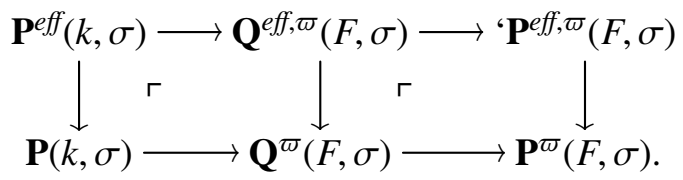

D'après [4, Prop. 2.108, (c)], le morphisme d'évaluation $\int: \mathbf{P}(k, \sigma) \longrightarrow \mathbb{C}$, donné par la composition de

$$
\mathbf{P}(k, \sigma) \hookrightarrow \mathbf{P}(k, \sigma) \otimes_{k} \mathbb{C} \simeq \mathbf{H}_{\text {mot }}(k, \sigma) \otimes_{\mathbb{Q}} \mathbb{C} \stackrel{\text { cu }}{\longrightarrow} \mathbb{C},
$$

envoie, modulo l'isomorphisme du Théorème 3.13, la classe d'une fonction $f \in \mathcal{O}_{k \text {-alg }}\left(\overline{\mathbb{D}}^{\infty}\right)$ sur son intégrale $\int_{[0,1]^{\infty}} f$.

Définition 3.25 - On pose :

$$
\mathbf{R}_{/ k}^{\varpi}(F, \sigma)=\mathbf{P}^{\varpi}(F, \sigma) \otimes_{\mathbf{P}(k, \sigma), \int} \mathbb{C} .
$$

C'est une $\left(F \otimes_{k} \mathbb{C}\right)$-algèbre.

Proposition 3.26 - Le pro-schéma $\operatorname{Spec}\left(\mathbf{R}_{l k}^{\varpi}(F, \sigma)\right)$ est naturellement un $\left(F \otimes_{k} \mathbb{C}\right)$-torseur sous le groupe de Galois motivique relatif $\mathbf{G}_{/ k}^{\mathrm{rel}, \varpi}(F, \sigma)$.

Demonstration Rappelons que $\operatorname{Spec}(\mathbf{P}(k, \sigma))$ est un $k$-torseur sous le groupe de Galois motivique $\mathbf{G}_{\text {mot }}(k, \sigma)$. De même, $\operatorname{Spec}\left(\mathbf{P}^{\varpi}(F, \sigma)\right)$ est un $F$-torseur sous le groupe de Galois motivique $\mathbf{G}_{\text {mot }}^{\varpi}(F, \sigma)$. D'après la Proposition 3.15, le morphisme canonique

$$
\operatorname{Spec}\left(\mathbf{P}^{\varpi}(F, \sigma)\right) \longrightarrow \operatorname{Spec}(\mathbf{P}(k, \sigma))
$$

est $\mathbf{G}_{\text {mot }}^{\bar{\sigma}}(F, \sigma)$-équivariant lorsque ce groupe agit sur $\operatorname{Spec}(\mathbf{P}(k, \sigma))$ par restriction suivant le morphisme canonique

$$
\mathbf{G}_{\mathrm{mot}}^{\varpi}(F, \sigma) \longrightarrow \mathbf{G}_{\mathrm{mot}}(k, \sigma) .
$$

Maintenant, le morphisme d'évaluation $\int: \mathbf{P}(k, \sigma) \longrightarrow \mathbb{C}$ définit un $\mathbb{C}$-point de $\operatorname{Spec}(\mathbf{P}(k, \sigma))$. Il en découle aussitôt que le produit fibré

$$
\operatorname{Spec}\left(\mathbf{P}^{\varpi}(F, \sigma)\right) \times_{\operatorname{Spec}(\mathbf{P}(k, \sigma))} \operatorname{Spec}(\mathbb{C}),
$$

vu comme $\left(F \otimes_{k} \mathbb{C}\right)$-schéma, est un torseur sous le noyau du morphisme (112). Ce noyau n'est autre que le groupe de Galois motivique relatif de l'extension $F / k$.

Vu le carré cocartésien extérieur du diagramme (108), on a en fait un isomorphisme canonique

$$
\mathbf{R}_{/ k}^{\varpi}(F, \sigma) \simeq{ }^{e f f, \varpi}(F, \sigma) \otimes_{\mathbf{P}^{e f f}(k, \sigma), \int} \mathbb{C}=\left(\mathbf{Q}^{e f f, \varpi}(F, \sigma) \otimes_{\mathbf{P}^{e f f}(k, \sigma), \int} \mathbb{C}\right) \otimes_{F} \widehat{F}[\ln \varpi] .
$$

On obtient alors le résultat suivant.

THÉORÈMe 3.27 - Le noyau de la composition de

$$
\mathcal{O}_{k-a l g}^{\dagger}\left(\overline{\mathbb{D}}^{\infty}\right) \longrightarrow \mathbf{P}^{\varpi}(F, \sigma) \longrightarrow \mathbf{R}_{/ k}^{\varpi}(F, \sigma)
$$

est le sous-F-espace vectoriel de $\mathcal{O}_{k-a l g}^{\dagger}\left(\overline{\mathbb{D}}^{\infty}\right)$ engendré par les éléments de la forme

$$
\frac{\partial G}{\partial z_{i}}-\left.G\right|_{z_{i}=1}+\left.G\right|_{z_{i}=0} \quad \text { et } \quad f \cdot L
$$

avec $G, L \in \mathcal{O}_{k \text {-alg }}^{\dagger}\left(\overline{\mathbb{D}}^{\infty}\right), i \in \mathbb{N} \backslash\{0\}$ et $f \in \mathcal{O}_{k \text {-alg }}\left(\overline{\mathbb{D}}^{\infty}\right)$ vérifiant $\int_{[0,1]^{\infty}} f=0$, et telle que $f$ et $L$ ne dépendent pas simultanément d'une même variable complexe. 
Demonstration Notons $\mathscr{P}(k)$ l'image du morphisme d'évaluation $\int: \mathcal{O}_{k-a l g}\left(\overline{\mathbb{D}}^{\infty}\right) \longrightarrow \mathbb{C}$. Puisque $\mathbb{C}$ est plat sur l'anneau $\mathscr{P}(k)$, le noyau de la composition (114) coïncide avec le noyau du morphisme

$$
\mathcal{O}_{k-a l g}^{\dagger}\left(\overline{\mathbb{D}}^{\infty}\right) \longrightarrow \mathbf{Q}^{e f f, \varpi}(F, \sigma) \otimes_{\mathbf{P}^{e f f}(k, \sigma)} \mathscr{P}(k) .
$$

Le résultat découle maintenant du Corollaire 3.23 .

\section{Réalisations et évaluations}

On garde les notations des sections précédentes. Ici, nous allons combiner les Théorèmes 3.10 et 3.27 pour déduire le Théorème 1.8. Pour ce faire, nous construisons un torseur sur la complétion pro-C-C-algébrique de $\pi_{1}^{\varpi}(\mathbb{C} \backslash \bar{k})$. L'algèbre des fonctions régulières sur ce torseur est une sous- $\left(F \otimes_{k} \mathbb{C}\right)$-algèbre $\widetilde{\mathcal{A}}_{F \otimes_{k} \mathbb{C}}^{\text {rs }}$ de $\mathbb{C}((\varpi))\left(\varpi^{1 / r} ; r \in \mathbb{N}^{\times}\right)[\ln \varpi]$. On construit ensuite un morphisme $\mathbf{R}_{/ k}^{\varpi}(F, \sigma) \longrightarrow \widetilde{\mathcal{A}}_{F \otimes_{k} \mathbb{C}}^{\mathrm{rs}}$ et on montre qu'il est donné par l'intégration (du moins pour les éléments venant de $\left.\mathbf{Q}^{\varpi}(F, \sigma)\right)$. Ce morphisme étant compatible à l'action du groupe $\pi_{1}^{\varpi}(\mathbb{C} \backslash \bar{k})$, on est en mesure de conclure grâce au Théorème 3.10.

On commence par des rappels sur la correspondance de Riemann-Hilbert (dans le cas particulier d'un ouvert Zariski de la droite affine).

4.1. Sur la correspondance de Riemann-Hilbert. - Soit $U \subset \mathbb{A}_{\mathbb{C}}^{1}$ un ouvert Zariski. On note $\mathcal{D}_{U}$ le faisceau (Zariski) des opérateurs différentiels sur $U$. En tant que $\mathcal{O}_{U}$-module quasi-cohérent, on a $\mathcal{D}_{U}=\mathcal{O}_{U}\left[\partial_{\varpi}\right]$ où $\partial_{\varpi}=\frac{\partial}{\partial \varpi}$. On note $\mathcal{O}-\mathbf{C o h}\left(\mathcal{D}_{U}\right)$ la catégorie des $\mathcal{D}_{U}$-modules à gauche qui sont cohérents en tant que $\mathcal{O}_{U}$-modules. De tels modules sont automatiquement holonomes sur $\mathcal{D}_{U}$ et localement libres sur $\mathcal{O}_{U}$ (voir [8, Chap. VI, Prop. 1.7]). La donnée d'un objet de $\mathcal{O}-\mathbf{C o h}\left(\mathcal{D}_{U}\right)$ équivaut à celle d'une connexion intégrable sur un $\mathcal{O}_{U}$-module localement libre. (La condition d'intégrabilité est vide dans notre cas puisque $\operatorname{dim}(U)=1$.) On note également $\mathcal{O}-\widehat{\mathbf{C o h}}\left(\mathcal{D}_{U}\right)$ la catégorie des $\mathcal{D}_{U}$-modules à gauche qui sont l'union filtrante de leurs sous- $\mathcal{D}_{U}$-modules cohérents $\operatorname{sur} \mathcal{O}_{U}$.

On pose $\mathcal{D}_{U^{a n}}=\mathcal{O}_{U^{a n}} \otimes_{\mathcal{O}_{U}} \mathcal{D}_{U}$. C'est le faisceau des opérateurs différentiels sur la variété complexe $U^{a n}$. On a encore $\mathcal{D}_{U^{a n}}=\mathcal{O}_{U^{a n}}\left[\partial_{\varpi}\right]$ en tant que $\mathcal{O}_{U^{a n}}$-module. Comme dans le cas algébrique, on note $\mathcal{O}-\mathbf{C o h}\left(\mathcal{D}_{U^{a n}}\right)\left(\right.$ resp. $\left.\mathcal{O}-\widehat{\mathbf{C o h}}\left(\mathcal{D}_{U^{a n}}\right)\right)$ la catégorie des $\mathcal{D}_{U^{a n}-\text { modules à gauche }}$

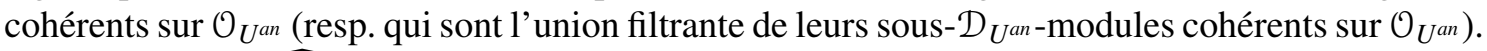

Rappelons que $\widehat{\mathbf{L S}}\left(U^{a n}, \mathbb{C}\right)$ est la catégorie des ind-systèmes locaux en $\mathbb{C}$-espaces vectoriels sur $U^{a n}$ (voir le paragraphe qui précède le Lemme 2.17). On dispose d'une équivalence de catégories

$$
\mathcal{O}_{U^{a n}} \otimes_{\mathbb{C}}-: \widehat{\mathbf{L S}}\left(U^{a n}, \mathbb{C}\right) \stackrel{\sim}{\longrightarrow} \mathcal{O}-\widehat{\mathbf{C o h}}\left(\mathcal{D}_{U^{a n}}\right)
$$

(voir [10, Chap. I, Th. 2.17]). Son quasi-inverse envoie un $\mathcal{D}_{U^{a n-m o d u l e}} \mathcal{M}$ sur le ind-système local $\operatorname{Sol}(\mathcal{M})=\operatorname{ker}\left\{\partial_{\varpi}: \mathcal{M} \rightarrow \mathcal{M}\right\}$. Par ailleurs, on dispose d'un foncteur d'《 analytification » :

$$
(-)^{a n}: \mathcal{O}-\widehat{\operatorname{Coh}}\left(\mathcal{D}_{U}\right) \longrightarrow \mathcal{O}-\widehat{\operatorname{Coh}}\left(\mathcal{D}_{U^{a n}}\right)
$$

Ce foncteur n'est pas une équivalence de catégories. Toutefois, si on le restreint à la sous-catégorie pleine $\mathcal{O}-\widehat{\mathbf{C o h}}_{\mathrm{rs}}\left(\mathcal{D}_{U}\right) \subset \mathcal{O}-\widehat{\mathbf{C o h}}\left(\mathcal{D}_{U}\right)$ formée des $\mathcal{D}_{U}$-modules qui sont l'union filtrante de leurs sous- $\mathcal{D}_{U}$-modules holonomes à singularités régulières (voir [10, Chap. II, §1] pour la définition), on obtient une équivalence de catégories

$$
(-)^{a n}: \mathcal{O}-\widehat{\mathbf{C o h}}_{\mathrm{rs}}\left(\mathcal{D}_{U}\right) \stackrel{\sim}{\longrightarrow} \mathcal{O}-\widehat{\mathbf{C o h}}\left(\mathcal{D}_{U^{a n}}\right)
$$

(voir [10, Chap. II, Th. 5.9]). Bien entendu, cette équivalence de catégories identifie $\mathcal{O}-\mathbf{C o h}\left(\mathcal{D}_{U^{a n}}\right)$ à la sous-catégorie pleine $\mathcal{O}-\mathbf{C o h}_{\mathrm{rs}}\left(\mathcal{D}_{U}\right) \subset \mathcal{O}-\mathbf{C o h}\left(\mathcal{D}_{U}\right)$ des $\mathcal{D}_{U}$-modules cohérents sur $\mathcal{O}_{U}$ et à singularités régulières. La correspondance de Riemann-Hilbert est l'équivalence composée

$$
\mathrm{RH}_{U}=\text { Sol } \circ(-)^{a n}: \mathcal{O}-\widehat{\mathbf{C o h}}_{\mathrm{rs}}\left(\mathcal{D}_{U}\right) \stackrel{\sim}{\longrightarrow} \widehat{\mathbf{L S}}\left(U^{a n}, \mathbb{C}\right) .
$$

On fixe un quasi-inverse qu'on désignera par $\mathrm{RH}_{U}^{-1}$. 
Nous utiliserons la correspondance de Riemann-Hilbert pour définir un foncteur monoïdal sur la catégorie des ind-systèmes locaux sur $U^{a n}$ à valeurs dans la catégorie des $\mathcal{O}_{U}$-modules plats.

Définition 4.1 - On note $\mathrm{F}_{r h}: \widehat{\mathbf{L S}}\left(U^{a n}, \mathbb{C}\right) \longrightarrow \operatorname{Mod}\left(\mathcal{O}_{U}\right)$ la composition de

$$
\widehat{\mathbf{L S}}\left(U^{a n}, \mathbb{C}\right) \stackrel{\mathrm{RH}_{U}^{-1}}{\longrightarrow} \mathcal{O}-\widehat{\mathbf{C o h}}_{\mathrm{rs}}\left(\mathcal{D}_{U}\right) \stackrel{\text { oub }}{\longrightarrow} \operatorname{Mod}\left(\mathcal{O}_{U}\right),
$$

où oub est le foncteur qui envoie un $\mathcal{D}_{U}$-module sur le $\mathcal{O}_{U}$-module sous-jacent.

Par ailleurs, on dispose du foncteur fibre

$$
\Psi_{U^{a n}}: \widehat{\mathbf{L S}}\left(U^{a n}, \mathbb{C}\right) \longrightarrow \operatorname{Mod}(\mathbb{C}) .
$$

Dans le reste du paragraphe, nous tâcherons de calculer le torseur des isomorphismes entre les foncteurs $\Psi_{U^{a n}}$ et $\mathrm{F}_{r h}$.

Pour $\epsilon>0$ un réel suffisamment petit, on note $D_{\epsilon}=\{z \in \mathbb{C} ;|z|<\epsilon\}$ et $D_{\epsilon}^{*}=D_{\epsilon} \backslash\{0\}$. Soit $r: \tilde{D}_{\epsilon}^{*} \longrightarrow D_{\epsilon}^{*}$ le revêtement universel de $D_{\epsilon}^{*}$. On prendra pour $\tilde{D}_{\epsilon}^{*} \subset \mathbb{C}$, l'image inverse de $D_{\epsilon}^{*}$ par l'exponentielle. On pose

$$
\mathcal{A}=\operatorname{colim}_{\epsilon>0} \mathcal{O}\left(\tilde{D}_{\epsilon}^{*}\right) .
$$

Remarque 4.2 - Par définition, on a $\mathcal{A}=\Psi_{U^{a n}} \mathcal{O}_{U^{a n}}$. En effet, $r^{*} \mathcal{O}_{D_{\epsilon}^{*}}=\mathcal{O}_{\tilde{D}_{\epsilon}^{*}}$ puisque $r$ est un homéomorphisme local. (Voir aussi la preuve du Théorème 2.18.)

Par abus de notation, $\mathcal{A}$ désignera aussi le $\mathcal{O}_{U}$-module quasi-cohérent donné par $\mathcal{A}(V)=\mathcal{A}$ pour tout ouvert non vide $V \subset U$. Le faisceau $\mathcal{A}$ est naturellement un $\mathcal{D}_{U}$-module à gauche. On notera $\mathcal{A}_{U}^{\text {rs }}$ le plus grand sous- $\mathcal{D}_{U}$-module de $\mathcal{A}$ qui est un objet de $\mathcal{O}-\widehat{\mathbf{C o h}}_{\mathrm{rs}}\left(\mathcal{D}_{U}\right)$.

Proposition 4.3 - Il existe un isomorphisme naturel en $E \in \widehat{\mathbf{L S}}\left(U^{a n}, \mathbb{C}\right)$ :

$$
\operatorname{hom}_{\operatorname{Mod}(\mathbb{C})}\left(\Psi_{U^{a n}} E, \mathbb{C}\right) \simeq \operatorname{hom}_{\widehat{\mathbf{L S}}\left(U^{a n}, \mathbb{C}\right)}\left(E, \operatorname{RH}_{U}\left(\mathcal{A}_{U}^{\mathrm{rs}}\right)\right) \text {. }
$$

Autrement dit, l'adjoint à droite du foncteur (120) existe et il envoie un $\mathbb{C}$-espace vectoriel $V$ sur l'ind-système local $V \otimes_{\mathbb{C}} \mathrm{RH}_{U}\left(\mathcal{A}_{U}^{\mathrm{rs}}\right)$.

Demonstration On note $\mathcal{E}=\mathrm{RH}_{U}^{-1}(E)$ et $\mathcal{E}^{a n}=\mathcal{O}_{U^{a n}} \otimes_{\mathbb{C}} E$ son analytifié.

Rappelons que $\Psi_{U^{a n}} E=\Gamma\left(\tilde{D}_{\epsilon}^{*}, r^{*} E\right)$. (Bien entendu, on suppose que $\epsilon$ est suffisamment petit de sorte que $D_{\epsilon}^{*} \subset U^{a n}$.) Par ailleurs, $r^{*} E$ est le sous-faisceau des solutions de $r^{*} \mathcal{E}^{a n}$ (vu comme fibré à connexion sur $\left.\tilde{D}_{\epsilon}^{*}\right)$. Puisque $\tilde{D}_{\epsilon}^{*}$ est contractile, il s'ensuit un isomorphisme canonique :

$$
\left(\Psi_{U^{a n}} E\right) \otimes_{\mathbb{C}} \mathcal{O}\left(\tilde{D}_{\epsilon}^{*}\right) \simeq \Gamma\left(\tilde{D}_{\epsilon}^{*}, r^{*} \varepsilon^{a n}\right) .
$$

Par ailleurs, on a un morphisme évident

$$
\mathcal{E} \longrightarrow \operatorname{colim}_{\epsilon>0} \Gamma\left(\tilde{D}_{\epsilon}^{*}, r^{*} \mathcal{E}^{a n}\right)
$$

où le membre de droite est considéré comme un faisceau constant sur $U$. En combinant (123) et (124), on obtient un morphisme canonique de $\mathcal{D}_{U}$-modules à gauche $\mathcal{E} \longrightarrow\left(\Psi_{U^{a n}} E\right) \otimes_{\mathbb{C}} \mathcal{A}$. Puisque $\mathcal{E}$ est dans $\mathcal{O}-\widehat{\mathbf{C o h}}_{\mathrm{rs}}\left(\mathcal{D}_{U}\right)$, on en déduit un morphisme

$$
\mathrm{y}_{E}: \mathcal{E} \longrightarrow\left(\Psi_{U^{a n}} E\right) \otimes_{\mathbb{C}} \mathcal{A}_{U}^{\mathrm{rs}} .
$$

On définit l'homomorphisme $(122)$ en envoyant $f:\left(\Psi_{U^{a n}} E\right) \longrightarrow \mathbb{C}$ sur $\operatorname{RH}_{U}\left(\left(f \otimes \operatorname{id}_{\mathcal{A}_{U}^{\text {rs }}}\right) \circ \mathrm{y}_{E}\right)$.

Par ailleurs, on dispose d'un morphisme canonique

$$
\Gamma\left(\tilde{D}_{\epsilon}^{*}, r^{*}\left(\mathcal{A}_{U}^{\mathrm{rs}}\right)^{a n}\right)=\Gamma\left(\tilde{D}_{\epsilon}^{*}, r^{*}\left(\mathcal{A}_{U}^{\mathrm{rs}} \otimes_{\mathcal{O}_{U}} \mathcal{O}_{U^{a n}}\right)\right) \longrightarrow \mathcal{O}\left(\tilde{D}_{\epsilon}^{*}\right)
$$

donné par la multiplication des fonctions sur $\tilde{D}_{\epsilon}^{*}$. (Remarquer que $\mathcal{A}_{U}^{\mathrm{rs}}(U)$ est contenu dans $\mathcal{O}\left(\tilde{D}_{\epsilon}^{*}\right) \subset$ $\mathcal{A}$ dès que $D_{\epsilon}^{*} \subset U^{a n}$.) En appliquant (123), on obtient alors un morphisme canonique

$$
\left(\Psi_{U^{a n}}\left(\mathrm{RH}_{U}\left(\mathcal{A}_{U}^{\mathrm{rs}}\right)\right)\right) \otimes_{\mathbb{C}} \mathcal{O}\left(\tilde{D}_{\epsilon}^{*}\right) \longrightarrow \mathcal{O}\left(\tilde{D}_{\epsilon}^{*}\right)
$$

En passant à la limite suivant $\epsilon>0$, on déduit un morphisme de $\mathcal{D}_{U}$-modules

$$
\left(\Psi_{U^{a n}}\left(\mathrm{RH}_{U}\left(\mathcal{A}_{U}^{\mathrm{rs}}\right)\right)\right) \otimes_{\mathbb{C}} \mathcal{A} \longrightarrow \mathcal{A}
$$


où l'opérateur différentiel $\partial_{\varpi}$ agit sur la source par l'identité sur le premier facteur et par la dérivation des fonctions sur le second facteur. Or, on a $\operatorname{ker}\left\{\partial_{\varpi}: \mathcal{A} \rightarrow \mathcal{A}\right\}=\mathbb{C}$. Le morphisme (128) induit donc une forme linéaire

$$
\delta: \Psi_{U^{a n}}\left(\mathrm{RH}_{U}\left(\mathcal{A}_{U}^{\mathrm{rs}}\right)\right) \longrightarrow \mathbb{C} .
$$

L'inverse de l'homomorphisme (122) envoie $g: E \longrightarrow \mathrm{RH}_{U}\left(\mathcal{A}_{U}^{\mathrm{rs}}\right) \operatorname{sur} \delta \circ \Psi_{U^{a n}}(g)$.

On laissera au lecteur le soin de vérifier que les deux homomorphismes construits ci-dessus sont inverses l'un de l'autre.

Corollaire $4.4-$ Notons $\pitchfork_{U^{a n}}: \operatorname{Mod}(\mathbb{C}) \longrightarrow \widehat{\mathbf{L S}}\left(U^{a n}, \mathbb{C}\right)$ l'adjoint à droite du foncteur $\Psi_{U^{a n} .}$ Il existe alors un isomorphisme canonique de $\mathcal{O}_{U}$-algèbres

$$
\mathrm{F}_{r h} \circ \pitchfork_{U^{a n}}(\mathbb{C}) \simeq \mathcal{A}_{U}^{\mathrm{rs}} .
$$

Demonstration C'est une conséquence immédiate de la Proposition 4.3.

Le Corollaire 4.4 décrit l'algèbre du torseur des isomorphismes de $\mathrm{F}_{r h}$ vers $\Psi_{U^{a n}}$. Toutefois, nous aurons besoin de décrire le torseur des isomorphismes de $\Psi_{U^{a n}}$ vers $\mathrm{F}_{r h}$. Pour cela, notons $\left(\mathcal{A}_{U}^{\text {rs }}\right)^{\vee}$ le dual de $\mathcal{A}_{U}^{\text {rs }}$ vu comme pro-objet de $\mathcal{O}-\mathbf{C o h}_{\mathrm{rs}}\left(\mathcal{D}_{U}\right)$. (Rappelons que si $\mathcal{M} \in \mathcal{O}-\mathbf{C o h}\left(\mathcal{D}_{U}\right)$, son dual $\mathcal{M}^{\vee}$ est donné par $\mathcal{H} \operatorname{com}\left(\mathcal{M}, \mathcal{O}_{U}\right)$ en tant que $\mathcal{O}_{U}$-module ; l'opérateur différentiel $\partial_{\varpi}$ agit sur une section globale $p: \mathcal{M} \rightarrow \mathcal{O}_{U}$ par la formule $\left(\partial_{\varpi} p\right)(-)=\partial_{\varpi}(p(-))-p\left(\partial_{\varpi}(-)\right)$.) La Proposition 4.3 admet la conséquence suivante.

Corollatre 4.5 - Il existe un isomorphisme naturel en $E \in \widehat{\mathbf{L S}}\left(U^{a n}, \mathbb{C}\right)$ :

$$
\Psi_{U^{a n}} E \simeq \operatorname{hom}_{\widehat{\mathbf{L S}}\left(U^{a n}, \mathbb{C}\right)}\left(\mathrm{RH}_{U}\left(\left(\mathcal{A}_{U}^{\mathrm{rs}}\right)^{\vee}\right), E\right) .
$$

Demonstration On peut supposer que $E$ est un système local, i.e., un objet de $\mathbf{L S}\left(U^{a n}, \mathbb{C}\right)$. Le cas général s'en déduit par passage aux ind-objets. Le foncteur de dualité induit alors un isomorphisme

$$
\operatorname{hom}_{\widehat{\mathbf{L S}}\left(U^{a n}, \mathbb{C}\right)}\left(\mathrm{RH}_{U}\left(\left(\mathcal{A}_{U}^{\mathrm{rs}}\right)^{\vee}\right), E\right) \simeq \operatorname{hom}_{\widehat{\mathbf{L S}}\left(U^{a n}, \mathbb{C}\right)}\left(E^{\vee}, \mathrm{RH}_{U}\left(\mathcal{A}_{U}^{\mathrm{rs}}\right)\right) .
$$

D'après la Proposition 4.3, le membre de droite s'identifie à hom $\operatorname{Mod}(\mathbb{C})\left(\Psi_{U^{a n}} E^{\vee}, \mathbb{C}\right)=\left(\Psi_{U^{a n}} E^{\vee}\right)^{\vee}$. Or, le foncteur $\Psi_{U^{a n}}$ commute à la dualité, ce qui permet de conclure.

THÉORÈme 4.6 - Le foncteur $\mathrm{F}_{r h}: \widehat{\mathbf{L S}}\left(U^{a n}, \mathbb{C}\right) \longrightarrow \operatorname{Mod}\left(\mathcal{O}_{U}\right)$ possède un adjoint à droite qu'on notera $\mathrm{G}_{r h}$. De plus, on a un isomorphisme canonique de $\mathcal{O}(U)$-algèbres

$$
\Psi_{U^{a n}} \circ \mathrm{G}_{r h}\left(\mathcal{O}_{U}\right) \simeq \mathcal{A}_{U}^{\mathrm{rs}}(U) .
$$

Demonstration L'existence de $\mathrm{G}_{r h}$ est assurée par les critères généraux d'existence d'adjoints à droite. En utilisant le Corollaire 4.5, on peut former la chaîne d'isomorphismes canoniques :

$$
\begin{gathered}
\Psi_{U^{a n}} \circ \mathrm{G}_{r h}\left(\mathcal{O}_{U}\right) \simeq \operatorname{hom}_{\widehat{\mathbf{L S}}\left(U^{a n}, \mathbb{C}\right)}\left(\operatorname{RH}_{U}\left(\left(\mathcal{A}_{U}^{\mathrm{rs}}\right)^{\vee}\right), \mathrm{G}_{r h}\left(\mathcal{O}_{U}\right)\right) \\
\simeq \operatorname{hom}_{\operatorname{Mod}\left(\mathcal{O}_{U}\right)}\left(\mathrm{F}_{r h} \circ \operatorname{RH}_{U}\left(\left(\mathcal{A}_{U}^{\mathrm{rs}}\right)^{\vee}\right), \mathcal{O}_{U}\right) \simeq \operatorname{hom}_{\operatorname{Mod}\left(\mathcal{O}_{U}\right)}\left(\left(\mathcal{A}_{U}^{\mathrm{rs}}\right)^{\vee}, \mathcal{O}_{U}\right) \simeq \mathcal{A}_{U}^{\mathrm{rs}}(U) .
\end{gathered}
$$

Le théorème est démontré.

Dans la suite, on confondra souvent le faisceau $\mathcal{A}_{U}^{\text {rs }}$ et l'anneau de ses sections globales $\mathcal{A}_{U}^{\mathrm{rs}}(U)$. Ceci est loisible car $U$ est un schéma affine.

On rappelle que $\pi_{1}^{w}\left(U^{a n}\right)$ désigne le groupe fondamental de $U^{a n}$ relativement au vecteur tangent $\frac{\partial}{\partial \varpi}$ en 0 . La complétion pro- $\mathbb{C}$-algébrique de $\pi_{1}^{\varpi}\left(U^{a n}\right)$ est le spectre de la $\mathbb{C}$-algèbre de Hopf $\mathcal{C}_{f}^{0}\left(\pi_{1}^{\sigma \omega}\left(U^{a n}\right), \mathbb{C}\right)$ formée des fonctions à valeurs dans $\mathbb{C}$ sur le groupe $\pi_{1}^{\varpi}\left(U^{a n}\right)$ dont l'orbite (pour l'action régulière) engendre un $\mathbb{C}$-espace vectoriel de dimension finie. La catégorie $\widehat{\mathbf{L S}}\left(U^{a n}, \mathbb{C}\right)$ est équivalente à la catégorie des comodules à gauche sur $\mathcal{C}_{f}^{0}\left(\pi_{1}^{\varpi}\left(U^{a n}\right), \mathbb{C}\right)$ et, modulo cette équivalence, le foncteur $\Psi_{U^{a n}}$ s'identifie au foncteur d'oubli. D'après [4, Lem. 1.54], il s'ensuit un isomorphisme de $\mathbb{C}$-algèbres de Hopf $\Psi_{U^{a n}}\left(\pitchfork_{U^{a n}} \mathbb{C}\right) \simeq \mathcal{C}_{f}^{0}\left(\pi_{1}^{\varpi}\left(U^{a n}\right), \mathbb{C}\right)$. 
Corollaire 4.7 - Le pro-schéma $\operatorname{Spec}\left(\mathcal{A}_{U}^{\mathrm{rs}}\right)$ est naturellement un U-torseur sous la complétion pro-C-algébrique de $\pi_{1}^{\varpi}\left(U^{a n}\right)$.

Demonstration D'après [4, Prop. 1.28], $\mathcal{A}_{U}^{\text {rs }}$ est un comodule sur l'algèbre de Hopf $\Psi_{U^{a n}}\left(\pitchfork_{U^{a n}} \mathbb{C}\right)$. Ceci fournit l'action de la complétion pro-C-algébrique de $\pi_{1}^{\varpi}\left(U^{a n}\right) \operatorname{sur} \operatorname{Spec}\left(\mathcal{A}_{U}^{\mathrm{rs}}\right)$. Pour montrer que ce dernier est un torseur, il suffit de montrer que les foncteurs $\mathrm{F}_{r h}$ et $\Psi_{U^{a n}}$ deviennent isomorphes (non canoniquement) après extension des scalaires aux points géométriques de $U$. La catégorie $\widehat{\mathbf{L S}}\left(U^{a n}, \mathbb{C}\right)$ est la catégorie des ind-objets dans la catégorie tannakienne $\mathbf{L S}\left(U^{a n}, \mathbb{C}\right)$, et les foncteurs $\Psi_{U^{a n}}(-)$ et $\mathrm{F}_{r h}$ commutent aux colimites filtrantes. Il suffit donc de montrer le résultat pour les restrictions de ces foncteurs à $\mathbf{L S}\left(U^{a n}, \mathbb{C}\right)$. C'est alors un cas particulier de l' «unicité » des foncteurs fibres pour les catégories tannakiennes. Nous invitons le lecteur à consulter [11] et notamment le $\S 9$ de loc. cit. pour plus de détails.

Plus tard, nous aurons besoin du résultat suivant.

Proposition 4.8 - Il existe un morphisme canonique de faisceaux sur $U^{\text {an }}$ :

$$
\mathrm{G}_{r h}\left(\mathcal{O}_{U}\right) \longrightarrow \mathcal{O}_{U^{a n}}
$$

Ce morphisme est injectif et $\mathcal{O}(U)$-linéaire. De plus, le carré suivant est commutatif

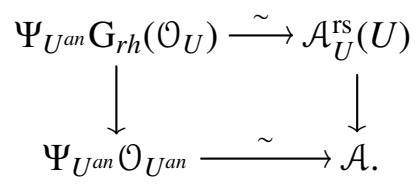

Demonstration Soit $E$ un ind-système local sur $U^{a n}$, et notons $\mathcal{E}=\mathrm{RH}_{U}^{-1}(E)$ et $\mathcal{E}^{a n}=\mathcal{O}_{U^{a n}} \otimes_{\mathbb{C}} E$. Il existe alors un morphisme canonique

$$
\operatorname{hom}_{\operatorname{Shv}\left(U^{a n}, \mathbb{C}\right)}\left(E, \mathrm{G}_{r h}\left(\mathcal{O}_{U}\right)\right) \longrightarrow \operatorname{hom}_{\operatorname{Shv}\left(U^{a n}, \mathbb{C}\right)}\left(E, \mathcal{O}_{U^{a n}}\right)
$$

donné par la composition de

$$
\begin{aligned}
\operatorname{hom}_{\operatorname{Shv}\left(U^{a n}, \mathbb{C}\right)}\left(E, \mathrm{G}_{r h}\left(\mathcal{O}_{U}\right)\right) \stackrel{\sim}{\longrightarrow} & \operatorname{hom}_{\operatorname{Mod}\left(\mathcal{O}_{U}\right)}\left(\mathcal{E}, \mathcal{O}_{U}\right) \\
& \operatorname{hom}_{\operatorname{Mod}\left(\mathcal{O}_{U^{a n}}\right)}\left(\mathcal{E}^{a n}, \mathcal{O}_{U^{a n}}\right) \stackrel{\sim}{\longrightarrow} \operatorname{hom} \operatorname{Shv}\left(U^{a n}, \mathbb{C}\right)\left(E, \mathcal{O}_{U^{a n}}\right) .
\end{aligned}
$$

Le morphisme (132) est l'image de l'identité de $\mathrm{G}_{r h}\left(\mathcal{O}_{U}\right)$ par (133).

Il reste à montrer que le carré de l'énoncé est commutatif (l'injectivité et la $\mathcal{O}(U)$-linéarité de (132) en découlent). Pour cela, considérons la composition de

$$
\operatorname{hom}_{\widehat{\mathbf{L S}}\left(U^{a n}, \mathbb{C}\right)}\left(\mathrm{RH}_{U}\left(\left(\mathcal{A}_{U}^{\mathrm{rs}}\right)^{\vee}\right), \mathrm{G}_{r h}\left(\mathcal{O}_{U}\right)\right) \simeq \Psi_{U^{a n}} \mathrm{G}_{r h}\left(\mathcal{O}_{U}\right) \longrightarrow \Psi_{U^{a n}} \mathcal{O}_{U^{a n}} \simeq \mathcal{A} .
$$

Nous allons calculer l'image d'un morphisme $f: \mathrm{RH}_{U}\left(\left(\mathcal{A}_{U}^{\mathrm{rs}}\right)^{\vee}\right) \longrightarrow \mathrm{G}_{r h}\left(\mathcal{O}_{U}\right)$ par cette composition. (Ce calcul est basé sur les preuves de la Proposition 4.3 et du Corollaire 4.5.) Notons $E \subset \mathrm{G}_{r h}\left(\mathcal{O}_{U}\right)$ l'image du morphisme $f$; c'est un système local contenu dans $\mathrm{G}_{r h}\left(\mathcal{O}_{U}\right)$. On a donc une factorisation de $f$ :

$$
\mathrm{RH}_{U}\left(\left(\mathcal{A}_{U}^{\mathrm{rs}}\right)^{\vee}\right) \stackrel{g}{\longrightarrow} E \hookrightarrow \mathrm{G}_{r h}\left(\mathcal{O}_{U}\right)
$$

On considère le morphisme $g^{\vee}: E^{\vee} \longrightarrow \mathrm{RH}_{U}\left(\mathcal{A}_{U}^{\mathrm{rs}}\right)$, dual du morphisme $g$. D'après la preuve de la Proposition 4.3, le morphisme $g^{\vee}$ correspond à un unique morphisme $h^{\vee}: \Psi_{U^{a n}}(E)^{\vee}=$ $\Gamma\left(\tilde{D}_{\epsilon}^{*}, r^{*} E^{\vee}\right) \longrightarrow \mathbb{C}$ tel que le diagramme

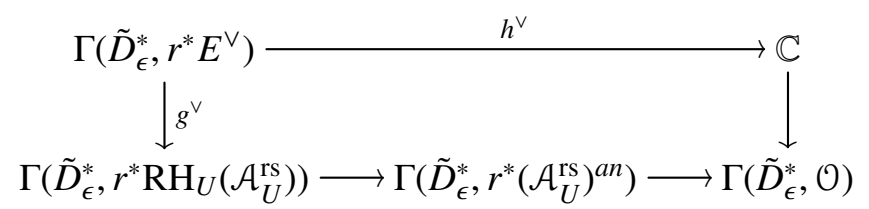


commute. Le dual de $h^{\vee}$ fournit une section $h \in \Gamma\left(\tilde{D}_{\epsilon}^{*}, r^{*} E\right)$. Alors, l'image de $f$ par la composition de (134) est égale à l'image de $h$ par la composition de

$$
E \longrightarrow \mathrm{G}_{r h}\left(\mathcal{O}_{U}\right) \longrightarrow \mathcal{O}_{U^{a n}}
$$

Par adjonction, la composition de (136) correspond à un morphisme $\mathcal{E}^{a n} \longrightarrow \mathcal{O}_{U^{a n}}$ et on considère le morphisme dual $\mathcal{O}_{U^{a n}} \longrightarrow \mathcal{E}^{a n, \vee}$. Il s'ensuit aussitôt que l'image de $h$ par (136) est l'image de $1 \in \Gamma\left(\tilde{D}_{\epsilon}^{*}, \mathcal{O}\right)$ par la composition de

$$
\Gamma\left(\tilde{D}_{\epsilon}^{*}, \mathcal{O}\right) \longrightarrow \Gamma\left(\tilde{D}_{\epsilon}^{*}, r^{*} \mathcal{E}^{a n, \vee}\right) \simeq \Gamma\left(\tilde{D}_{\epsilon}^{*}, r^{*} E^{\vee}\right) \otimes_{\mathbb{C}} \Gamma\left(\tilde{D}_{\epsilon}^{*}, \mathcal{O}\right) \stackrel{h^{\vee}}{\longrightarrow} \Gamma\left(\tilde{D}_{\epsilon}^{*}, \mathcal{O}\right) .
$$

Vu le diagramme commutatif (135), la composition ci-dessus est égale à celle de

$$
\Gamma\left(\tilde{D}_{\epsilon}^{*}, \mathcal{O}\right) \longrightarrow \Gamma\left(\tilde{D}_{\epsilon}^{*}, r^{*} \varepsilon^{a n, \vee}\right) \stackrel{g^{a n, \vee}}{\longrightarrow} \Gamma\left(\tilde{D}_{\epsilon}^{*}, r^{*}\left(\mathcal{A}_{U}^{\mathrm{rs}}\right)^{a n}\right) \longrightarrow \Gamma\left(\tilde{D}_{\epsilon}^{*}, \mathcal{O}\right) .
$$

Le morphisme $f: \mathrm{RH}_{U}\left(\left(\mathcal{A}_{U}^{\mathrm{rs}}\right)^{\vee}\right) \longrightarrow \mathrm{G}_{r h}\left(\mathcal{O}_{U}\right)$ correspond par adjonction à un morphisme de $\mathcal{O}_{U}$-modules $f^{\prime}:\left(\mathcal{A}_{U}^{\mathrm{rs}}\right)^{\vee} \longrightarrow \mathcal{O}_{U}$. En dualisant, on obtient une section globale $f^{\prime \vee} \in \mathcal{A}_{U}^{\mathrm{rs}}(U)$. De même, la composition de

$$
\mathrm{RH}_{U}\left(\left(\mathcal{A}_{U}^{\mathrm{rs}}\right)^{\vee}\right) \longrightarrow \mathrm{G}_{r h}\left(\mathcal{O}_{U}\right) \longrightarrow \mathcal{O}_{U^{a n}}
$$

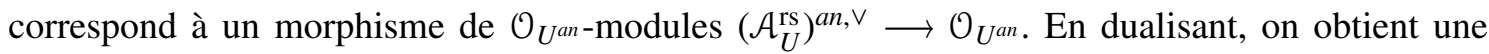
section globale $f^{\prime a n, \vee} \in\left(\mathcal{A}_{U}^{\mathrm{rs}}\right)^{a n}\left(U^{a n}\right)$. D'après la construction du morphisme $\mathrm{G}_{r h}\left(\mathcal{O}_{U}\right) \longrightarrow \mathcal{O}_{U^{a n}}$, il est immédiat que $f^{\prime a n, \vee}$ est l'image de $f^{\prime \vee}$ par le morphisme évident $\mathcal{A}_{U}^{\mathrm{rs}}(U) \longrightarrow\left(\mathcal{A}_{U}^{\mathrm{rs}}\right)^{a n}\left(U^{a n}\right)$. Par ailleurs, il est clair que $f^{\prime a n, \vee}$ est aussi l'image de 1 par la composition des deux premières flèches dans (138). Or, le morphisme $\Gamma\left(\tilde{D}_{\epsilon}^{*}, r^{*}\left(\mathcal{A}_{U}^{\mathrm{rs}}\right)^{a n}\right) \longrightarrow \Gamma\left(\tilde{D}_{\epsilon}^{*}, \mathcal{O}\right)$ envoie $f^{\prime a n, \vee}=\left(f^{\prime \vee}\right)^{a n}$ sur $f^{\prime \vee}$ (lorsqu'on identifie $\mathcal{A}_{U}^{\text {rs }}(U)$ à un sous-anneau de $\Gamma\left(\tilde{D}_{\epsilon}^{*}, \mathcal{O}\right)$ ). On a donc démontré que l'image de $f$ par la composition de (134) est égale à l'image de $f^{\prime \vee}$ par l'inclusion $\mathcal{A}_{U}^{\mathrm{rs}}(U) \hookrightarrow \mathcal{A}$. Ceci établit la commutation du carré de l'énoncé.

4.2. Réalisation de Betti et connexions de Gauss-Manin. - Nous allons d'abord étendre la connexion de Gauss-Manin aux motifs. Soit $U \subset \mathbb{A}_{\mathbb{C}}^{1}$ un ouvert non vide et notons $\Omega_{j U}^{\cdot}$ le complexe de faisceaux (avec transferts, d'après [17]) sur $\mathrm{Sm} / U$ qui envoie un $U$-schéma lisse $X$ sur le complexe $\Gamma\left(X, \Omega_{X / U}^{\circ}\right)$ des sections globales du complexe de De Rham relatif $\Omega_{X / U}^{\cdot}$. On a une suite exacte courte de complexes de faisceaux avec transferts sur $\mathrm{Sm} / U$ :

$$
0 \longrightarrow \Omega_{/ U}^{\cdot}[-1] \longrightarrow\left(\Omega_{/ \mathbb{C}}^{\bullet}\right)_{\text {Sm/U }} \longrightarrow \Omega_{/ U}^{\cdot} \longrightarrow 0
$$

où la première flèche non nulle envoie $v \in \Omega_{/ U}^{\circ}(X)$ sur $\mathrm{d} \varpi \wedge v$ et la seconde flèche non nulle envoie $u \in \Omega_{/ \mathbb{C}}^{\bullet}(X)$ sur sa classe dans $\Omega_{/ U}^{\cdot}(X)$. On note

$$
\partial_{\varpi}: \Omega_{/ U}^{\bullet} \longrightarrow \Omega_{/ U}^{\bullet}
$$

le morphisme de $\mathbf{D M}^{e f f}(U)$ défini comme étant l'opposé du morphisme déduit de la suite exacte (139). Ainsi,

$$
\Omega_{/ U}^{\bullet}[-1] \longrightarrow\left(\Omega_{/ \mathbb{C}}^{\bullet}\right)_{\operatorname{Sm} / U} \longrightarrow \Omega_{/ U}^{\cdot} \stackrel{-\partial_{\varpi}}{\longrightarrow} \Omega_{/ U}^{\bullet}
$$

est un triangle distingué dans $\mathbf{D M}^{e f f}(U)$. On a le résultat suivant.

Proposition 4.9 - L'endomorphisme (140) vérifie la formule de Leibniz, i.e.,

$$
\partial_{\varpi} \circ \mathrm{m}_{f}=\mathrm{m}_{f} \circ \partial_{\varpi}+\mathrm{m}_{\partial_{\varpi} f}
$$

où $\mathrm{m}_{f}$ désigne la multiplication par une fonction régulière $f$ sur $U$.

Demonstration Notons $\mathcal{K}^{\bullet}=\operatorname{Cône}\left\{\left.\Omega_{/ U}^{\cdot}[-1] \rightarrow\left(\Omega_{/ \mathbb{C}}\right)\right|_{\mathrm{Sm} / U}\right\}$. Ainsi, pour $X \in \mathrm{Sm} / U$, on a $\mathcal{K}^{n}(X)=\Omega_{/ \mathbb{C}}^{n}(X) \oplus \Omega_{/ U}^{n}(X)$ et la différentielle est donnée par

$$
\mathrm{d}_{\mathcal{K}}(u, v)=(\mathrm{d} u+\mathrm{d} \varpi \wedge v, \mathrm{~d} v)
$$


pour $u \in \Omega_{/ \mathbb{C}}^{n}(X)$ et $v \in \Omega_{/ U}^{n}(X)$. On dispose aussi de deux morphismes de complexes $\mathrm{p}, \mathrm{q}$ : $\mathcal{K}^{\bullet} \longrightarrow \Omega_{/ U}^{\bullet}$ donnés par

$$
\mathrm{p}(u, v)=\bar{u} \quad \text { et } \quad \mathrm{q}(u, v)=v .
$$

(Dans la première équation ci-dessus, $\bar{u}$ désigne la classe de $u$ dans $\Omega_{/ U}^{n}(X)$.) Vu la suite exacte (139), le morphisme $\mathrm{p}: \mathcal{K}^{\bullet} \longrightarrow \Omega_{j U}^{\bullet}$ est un quasi-isomorphisme de complexes de faisceaux et on a la formule $\partial_{\varpi}=-\mathrm{q} \circ \mathrm{p}^{-1}$ dans la catégorie dérivée des complexes de faisceaux avec transferts sur $\mathrm{Sm} / U$.

Pour $f \in \mathcal{O}(U)$, la formule $\mathrm{n}_{f}(u, v)=\left(f \cdot u, f \cdot v-f^{\prime} \cdot \bar{u}\right)$ définit un endomorphisme $\mathrm{n}_{f} \mathrm{du}$ complexe $\mathcal{K}^{\bullet}$. (Ici, on a posé $f^{\prime}=\partial_{\varpi} f$.) De plus, on a clairement les relations $\mathrm{p} \circ \mathrm{n}_{f}=\mathrm{m}_{f} \circ \mathrm{p}$ et $\mathrm{q} \circ \mathrm{n}_{f}=\mathrm{m}_{f} \circ \mathrm{q}-\mathrm{m}_{f^{\prime}} \circ \mathrm{p}$. Ceci permet de conclure.

Remarque 4.10 - L'analogue analytique de la suite exacte courte (139) fournit aussi un morphisme $\partial_{\varpi}: \Omega_{/ U^{a n}} \longrightarrow \Omega^{\circ}{ }_{U^{a n}}$ dans la catégorie dérivée des faisceaux sur le site $\mathrm{AnSm} / U^{a n}$ des espaces analytiques lisses sur $U^{a n}$ et on a un carré commutatif

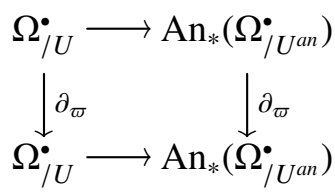

dans $\mathbf{D M}^{\text {eff }}(U)$. (Rappelons que An $: \mathrm{Sm} / U \longrightarrow \mathrm{AnSm} / U^{a n}$ est le foncteur d'analytification.) La preuve de la Proposition 4.9 s'étend littéralement pour montrer que $\partial_{\varpi}: \Omega_{/ U^{a n}} \longrightarrow \Omega_{/ U^{a n}}$ satisfait aussi à la formule de Leibniz. On peut donner une preuve plus conceptuelle de cela. C'est la suivante. Notons $\iota: \operatorname{Ouv}\left(U^{a n}\right) \hookrightarrow \mathrm{AnSm} / U^{a n}$ l'inclusion de l'ensemble ordonné des ouverts de $U^{a n}$. On dispose d'un morphisme canonique $\iota^{*} \mathcal{O}_{U^{a n}} \longrightarrow \Omega_{/ U^{a n}}^{*}$ et le lemme de Poincaré holomorphe entraîne que c'est un quasi-isomorphisme de préfaisceaux. Or, il est facile de voir que le carré

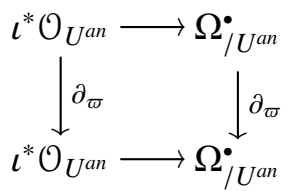

est commutatif, ce qui permet de conclure.

D'après la Proposition 4.9, le $\mathcal{O}(U)$-module $\mathrm{dR}_{U}(M)=\operatorname{hom}_{\mathbf{D M}^{e f f}(F)}\left(M, \Omega_{/ U}^{\bullet}\right)$ hérite d'une structure de $\mathcal{D}(U)$-module à gauche pour tout $M \in \mathbf{D M}^{\text {eff }}(U)$. Lorsque $M$ devient fortement dualisable dans $\mathbf{D M}(U)$, ce $\mathcal{D}(U)$-module est projectif sur $\mathcal{O}(U)$ et il définit alors un objet de $\mathcal{O}-\mathbf{C o h}_{\mathrm{rs}}(U)$. (Le fait que le $\mathcal{D}_{U}$-module en question est à singularités régulières est une conséquence facile de [10, Chap. II, Th. 7.9].) La connexion associée est la connexion de Gauss-Manin bien connue. En général, nous obtenons un foncteur

$$
\mathrm{dR}_{U}^{G M}: \mathbf{D M}^{\text {eff }}(U) \longrightarrow \operatorname{Mod}(\mathcal{D}(U))^{\mathrm{op}}
$$

qui « enrichit » le foncteur $\mathrm{dR}_{U}: \mathbf{D M}^{\text {eff }}(U) \longrightarrow \operatorname{Mod}(\mathcal{O}(U))^{\text {op }}$ envoyant $M$ sur le $\mathcal{O}(U)$-module $\operatorname{hom}_{\mathbf{D M}{ }^{e f f}(F)}\left(M, \Omega_{/ U}^{\cdot}\right)$.

THÉorème 4.11 - Il existe un isomorphisme naturel en $M \in \operatorname{lisDM}^{e f f}(U)$ :

$$
\mathrm{dR}_{U}^{G M}(M) \simeq \operatorname{hom}_{M o d\left(\mathcal{O}_{U}\right)}\left(\mathrm{RH}_{U}^{-1}\left(\mathrm{H}_{0} \operatorname{Bti}_{U}^{*}(M) \otimes_{\mathbb{Q}} \mathbb{C}\right), \mathcal{O}_{U}\right)
$$

où le membre de droite est muni de sa structure de $\mathcal{D}(U)$-module déduite de la structure de $\mathcal{D}_{U}$ module sur $\mathrm{RH}_{U}^{-1}\left(\mathrm{H}_{0} \mathrm{Bti}_{U}^{*}(M) \otimes_{\mathbb{Q}} \mathbb{C}\right)$.

Demonstration On pose $\mathrm{dR}_{U^{a n}}(M)=\operatorname{hom}_{\mathbf{D M}^{e f f}(U)}\left(M, \mathrm{An}_{*} \Omega_{/ U^{a n}}\right)$. D'après la Remarque 4.10 , ce $\mathcal{O}\left(U^{a n}\right)$-module est naturellement un $\mathcal{D}\left(U^{a n}\right)$-module qu'on notera $\mathrm{dR}_{U^{a n}}^{G M}(M)$. Lorsque $M$ devient 
fortement dualisable dans $\mathbf{D M}(U), \mathrm{dR}_{U^{a n}}^{G M}(M)$ est projectif sur $\mathcal{O}\left(U^{a n}\right)$ et définit donc un objet de $\mathcal{O}-\operatorname{Coh}\left(\mathcal{D}_{U^{a n}}\right)$. En général, on a des isomorphismes canoniques de $\mathcal{D}\left(U^{a n}\right)$-modules

$$
\begin{gathered}
\mathrm{dR}_{U^{a n}}^{G M}(M)=\operatorname{hom}_{\mathbf{D M}^{e f f}(U)}\left(M, \operatorname{An}_{*} \Omega_{/ U^{a n}}^{*}\right) \simeq \operatorname{hom}_{\mathbf{D M}^{e f f}(U)}\left(M, \operatorname{RAn}_{*} \iota^{*} \mathcal{O}_{U^{a n}}\right) \\
\simeq \operatorname{hom}_{\mathbf{A n D M}^{e f f}\left(U^{a n}\right)}\left(\operatorname{LAn}^{*} M, \iota^{*} \mathcal{O}_{U^{a n}}\right) \simeq \operatorname{hom}_{\mathbf{D}\left(U^{a n}\right)}\left(\operatorname{R}_{\iota_{*}} \operatorname{LAn}^{*} M, \mathcal{O}_{U^{a n}}\right) \\
=\operatorname{hom}_{\mathbf{D}\left(U^{a n}\right)}\left(\operatorname{Bti}_{U^{*}}^{*} M, \mathcal{O}_{U^{a n}}\right) \simeq \operatorname{hom}_{\mathbf{D}\left(\operatorname{Mod}\left(\mathcal{O}_{\left.U^{a n}\right)}\right)\right.}\left(\mathcal{O}_{U^{a n}} \otimes_{\mathbb{Q}} \operatorname{Bti}_{U}^{*}(M), \mathcal{O}_{U^{a n}}\right) \\
\simeq \operatorname{hom}_{\operatorname{Mod}\left(\mathcal{O}_{\left.U^{a n}\right)}\right.}\left(\mathcal{O}_{U^{a n}} \otimes_{\mathbb{Q}} \mathrm{H}_{0} \operatorname{Bti}_{U}^{*}(M), \mathcal{O}_{U^{a n}}\right) .
\end{gathered}
$$

Ci-dessus, nous avons utilisé plusieurs faits que nous allons maintenant lister. D'abord, nous avons utilisé que le morphisme évident $\iota^{*} \mathcal{O}_{U^{a n}} \longrightarrow \Omega_{/ U^{a n}}$ est un quasi-isomorphisme de complexes de faisceaux qui commute aux opérateurs $\partial_{\varpi}$. Nous avons aussi utilisé que le foncteur $\iota^{*}: \mathbf{D}\left(U^{a n}\right) \longrightarrow \mathbf{A n D M}^{e f f}\left(U^{a n}\right)$, induit par l'inclusion $\iota: \operatorname{Ouv}\left(U^{a n}\right) \hookrightarrow \mathrm{AnSm} / U^{a n}$, est une équivalence de catégories de quasi-inverse $\boldsymbol{R}_{\iota_{*}}$. Enfin, nous avons utilisé que les groupes $\operatorname{Ext}^{i}(\mathcal{M}, \mathcal{N})$

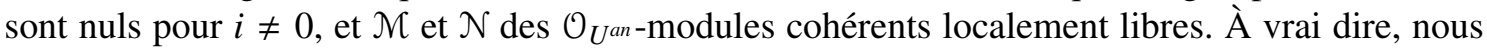
avons besoin de cette propriété dans un cadre un peu plus général : celui où $\mathcal{M}$ est une colimite

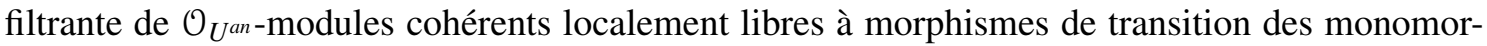
phismes scindés. (On se retrouve dans cette situation en écrivant les $\mathrm{H}_{n} \mathrm{Bti}_{U}^{*}(M)$ comme unions filtrantes de leurs sous-systèmes locaux.) Pour un tel $\mathcal{M}$, l'annulation des groupes $\operatorname{Ext}^{i}(\mathcal{M}, \mathcal{N})$ est une conséquence du critère de Mittag-Leffler (voir [14, Chap. 0, §13]).

À présent, on affirme que le morphisme de $\mathcal{D}(U)$-modules

$$
\mathrm{dR}_{U}^{G M}(M) \longrightarrow \mathrm{dR}_{U^{a n}}^{G M}(M)
$$

est injectif. Pour montrer cela, on écrit $M$ comme une colimite homotopique filtrante d'objets compacts $\left(M_{i}\right)_{i \in I}$ de lisDM ${ }^{e f f}(U)$. (Plus précisément, $I$ est un ensemble ordonné filtrant et $\left(M_{i}\right)_{i \in I}$ est un système inductif de complexes de préfaisceaux avec transferts sur $\mathrm{Sm} / U$ tel que chaque $M_{i}$ est fortement dualisable dans $\mathbf{D M}(U)$.) Pour tout $i \in I, \mathrm{dR}_{U}^{G M}\left(M_{i}\right)\left(\operatorname{resp} \cdot \mathrm{dR}_{U^{a n}}^{G M}\left(M_{i}\right)\right)$ est un $\mathcal{D}(U)$ module (resp. $\mathcal{D}\left(U^{a n}\right)$-module) projectif et de type fini sur $\mathcal{O}(U)$ (resp. $\mathcal{O}\left(U^{a n}\right)$ ). C'est donc un objet artinien de la catégorie des $\mathcal{D}(U)$-modules (resp. $\mathcal{D}\left(U^{a n}\right)$-modules). Il s'ensuit aussitôt que les systèmes projectifs $\left(\mathrm{dR}_{U}^{G M}\left(M_{i}\right)\right)_{i \in I}$ et $\left(\mathrm{dR}_{U^{a n}}^{G M}\left(M_{i}\right)\right)_{i \in I}$ vérifient la condition de Mittag-Leffler. On en déduit alors des isomorphismes canoniques

$$
\mathrm{dR}_{U}^{G M}(M)=\lim _{i \in I} \mathrm{dR}_{U}^{G M}\left(M_{i}\right) \quad \text { et } \quad \mathrm{dR}_{U^{a n}}^{G M}(M)=\lim _{i \in I} \mathrm{dR}_{U^{a n}}^{G M}\left(M_{i}\right) .
$$

Ainsi, pour montrer que (144) est injectif, on peut supposer que $M$ est compact. Dans ce cas, on a une identification $\mathrm{dR}_{U}(M) \otimes_{\mathcal{O}(U)} \mathcal{O}\left(U^{a n}\right) \simeq \mathrm{dR}_{U^{a n}}(M)$. Ceci permet de conclure puisque $\mathcal{O}\left(U^{a n}\right)$ est une $\mathcal{O}(U)$-algèbre fidèlement plate.

Considérons ensuite l'inclusion

$$
\operatorname{hom}_{\operatorname{Mod}\left(\mathcal{O}_{U}\right)}\left(\mathrm{RH}_{U}^{-1}\left(\mathrm{H}_{0} \mathrm{Bti}_{U}^{*}(M) \otimes_{\mathbb{Q}} \mathbb{C}\right), \mathcal{O}_{U}\right) \subset \operatorname{hom}_{\operatorname{Mod}\left(\mathcal{O}_{U^{a n}}\right)}\left(\mathcal{O}_{U^{a n}} \otimes_{\mathbb{Q}} \mathrm{H}_{0} \operatorname{Bti}_{U}^{*}(M), \mathcal{O}_{U^{a n}}\right) \text {. }
$$

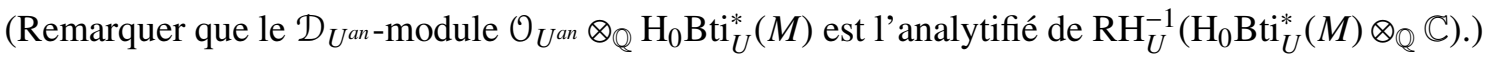
Nous allons montrer que l'isomorphisme composé de (143) et son inverse respectent les inclusions (144) et (146); ceci terminera la preuve du théorème. En utilisant (145) on peut supposer que $M$ devient fortement dualisable dans $\mathbf{D M}(U)$. Le résultat recherché est alors automatique : en effet les inclusions (144) et (146) sont de la forme $\Gamma(U, \mathcal{M}) \subset \Gamma\left(U^{a n}, \mathcal{M}^{a n}\right)$ avec $\mathcal{M} \in \mathcal{O}-\mathbf{C o h}_{\mathrm{rs}}\left(\mathcal{D}_{U}\right)$. Or, ces inclusions sont fonctorielles en $\mathcal{M}^{a n}$.

CorollaIre 4.12 - Il existe un isomorphisme naturel en $M \in \operatorname{lisDM}^{\text {eff }}(U)$ :

$$
\mathrm{dR}_{U}(M) \simeq \operatorname{hom}_{\mathbf{D}\left(U^{a n}\right)}\left(\operatorname{Bti}_{U}^{*}(M), \mathrm{G}_{r h}\left(\mathcal{O}_{U}\right)\right) .
$$

Demonstration D'après le Théorème 4.11 et les définitions des foncteurs $\mathrm{F}_{r h}$ et $\mathrm{G}_{r h}$, on a une chaîne d'isomorphismes

$$
\mathrm{dR}_{U}(M) \simeq \operatorname{hom}_{\operatorname{Mod}\left(\mathcal{O}_{U}\right)}\left(\mathrm{F}_{r h}\left(\mathrm{H}_{0} \mathrm{Bti}_{U}^{*}(M) \otimes_{\mathbb{Q}} \mathbb{C}\right), \mathcal{O}_{U}\right)
$$


Par ailleurs, on a un morphisme évident

$$
\simeq \operatorname{hom}_{\widehat{\mathbf{L S}}\left(U^{a n}\right)}\left(\mathrm{H}_{0} \mathrm{Bti}_{U}^{*}(M), \mathrm{G}_{r h}\left(\mathcal{O}_{U}\right)\right)
$$

$$
\operatorname{hom}_{\mathbf{D}_{\widehat{\mathbf{L}}}\left(U^{a n}\right)}\left(\mathrm{Bti}_{U}^{*}(M), \mathrm{G}_{r h}\left(\mathcal{O}_{U}\right)\right) \longrightarrow \operatorname{hom}_{\widehat{\mathbf{L S}}\left(U^{a n}\right)}\left(\mathrm{H}_{0} \mathrm{Bti}_{U}^{*}(M), \mathrm{G}_{r h}\left(\mathcal{O}_{U}\right)\right)
$$

On montrera que (149) est inversible, ce qui terminera la preuve du corollaire. Pour cela, il suffit de montrer que le groupe $\operatorname{hom}_{\mathbf{D}\left(U^{a n}\right)}\left(E, \mathrm{G}_{r h}\left(\mathcal{O}_{U}\right)[i]\right)$ est nul pour tout $E \in \widehat{\mathbf{L S}}\left(U^{a n}\right)$ et $i \in \mathbb{Z} \backslash\{0\}$. Si $i \notin$ $\{0,1\}$, ce groupe est nul puisque $U^{a n}$ a le type d'homotopie d'un bouquet de cercles. Lorsque $i=1$, le groupe en question s'identifie à $\operatorname{Ext}^{1}\left(E \otimes_{\mathbb{Q}} \mathbb{C}, \mathrm{G}_{r h}\left(\mathcal{O}_{U}\right)\right)$ calculé dans la catégorie $\widehat{\mathbf{L S}}\left(U^{a n}, \mathbb{C}\right)$. Or, le foncteur $\operatorname{hom}_{\widehat{\mathbf{L S}}\left(U^{a n}, \mathbb{C}\right)}\left(-, \mathrm{G}_{r h}\left(\mathcal{O}_{U}\right)\right) \simeq \operatorname{hom}_{\operatorname{Mod}\left(\mathcal{O}_{U}\right)}\left(\mathrm{F}_{r h}(-), \mathcal{O}_{U}\right)$ est exact. Il s'ensuit que $\mathrm{G}_{r h}\left(\mathcal{O}_{U}\right)$ est un objet injectif de $\widehat{\mathbf{L S}}\left(U^{a n}, \mathbb{C}\right)$. Ceci entraîne l'annulation recherchée.

4.3. Démonstration du théorème principal. - On travaille de nouveau au-dessus du corps $k$ muni du plongement complexe $\sigma: k \hookrightarrow \mathbb{C}$. Si $U \subset \mathbb{A}_{k}^{1}$ est un ouvert non vide, on notera $U_{\mathbb{C}}=U \otimes_{k} \mathbb{C}$. Dans ce paragraphe nous allons construire un morphisme de $\left(F \otimes_{k} \mathbb{C}\right)$-schémas

$$
\lim _{\emptyset \neq U \subset \mathbb{A}_{k}^{1}} \operatorname{Spec}\left(\mathcal{A}_{U_{\mathrm{C}}}^{\mathrm{rs}}\right) \longrightarrow \operatorname{Spec}\left(\mathbf{R}_{/ k}^{\varpi}(F, \sigma)\right)
$$

qui est $\pi_{1}^{\varpi}(\mathbb{C} \backslash \bar{k})$-équivariant. Le morphisme induit sur les algèbres de fonctions régulières est alors nécessairement injectif (cf. le Corollaire 4.24). Il restera à expliciter ce morphisme pour obtenir notre résultat principal. Cette tâche qui s'avérera fort pénible sera reléguée aux trois derniers paragraphes de l'article. On commence par adapter et réinterpréter le Corollaire 4.12.

Corollaire 4.13 - Soit $U \subset \mathbb{A}_{k}^{1}$ un ouvert non vide. Il existe un isomorphisme naturel en $M \in \operatorname{lisDM}^{e f f}(U)$ :

$$
\operatorname{hom}_{\mathbf{D M}^{e f f}(U)}\left(M, \Omega_{/ U}^{\bullet} \otimes_{k} \mathbb{C}\right) \simeq \operatorname{hom}_{\mathbf{D M}^{e f f}(U)}\left(M, \operatorname{Bti}_{U *} \mathrm{G}_{r h}\left(\mathcal{O}_{U_{\mathbb{C}}}\right)\right) .
$$

Demonstration D'après le Corollaire 4.12, et puisque $\mathrm{Bti}_{U}^{*} \simeq \mathrm{Bti}_{U_{\mathbb{C}}}^{*} \circ\left(U_{\mathbb{C}} / U\right)^{*}$, on a un isomorphisme canonique

$$
\operatorname{hom}_{\mathbf{D M}^{e f f}\left(U_{\mathbb{C}}\right)}\left(\left(U_{\mathbb{C}} / U\right)^{*} M, \Omega_{/ U_{\mathbb{C}}}^{\cdot}\right) \simeq \operatorname{hom}_{\mathbf{D}\left(U^{a n}\right)}\left(\operatorname{Bti}_{U}^{*}(M), \mathrm{G}_{r h}\left(\mathcal{O}_{U_{\mathbb{C}}}\right)\right) .
$$

En utilisant les adjonctions $\left(\left(U_{\mathbb{C}} / U\right)^{*},\left(U_{\mathbb{C}} / U\right)_{*}\right)$ et $\left(\mathrm{Bti}_{U}^{*}, \mathrm{Bti}_{U *}\right)$, on déduit un isomorphisme canonique

$$
\operatorname{hom}_{\mathbf{D M}^{e f f}(U)}\left(M,\left(U_{\mathbb{C}} / U\right)_{*} \Omega_{/ U_{\mathbb{C}}}\right) \simeq \operatorname{hom}_{\mathbf{D M}^{e f f}(U)}\left(M, \operatorname{Bti}_{U *} G_{r h}\left(\mathcal{O}_{U_{\mathbb{C}}}\right)\right) .
$$

On termine en remarquant que le morphisme canonique $\Omega_{/ U}^{\cdot} \otimes_{k} \mathbb{C} \longrightarrow\left(U_{\mathbb{C}} / U\right)_{*} \Omega_{/ U_{\mathbb{C}}}$ est un isomorphisme de complexes de préfaisceaux.

Pour un schéma $X$, on note $\operatorname{lis}_{X}: \mathbf{D M}^{e f f}(X) \longrightarrow \operatorname{lisDM}^{e f f}(X)$ l'adjoint à droite de l'inclusion évidente.

Corollaire $4.14-$ Soit $U \subset \mathbb{A}_{k}^{1}$ un ouvert non vide. Il existe un isomorphisme canonique dans $\operatorname{lisDM}^{e f f}(U)$ :

$$
\operatorname{lis}_{U}\left(\Omega_{/ U}^{\bullet} \otimes_{k} \mathbb{C}\right) \simeq \operatorname{lis}_{U}\left(\operatorname{Bti}_{U *} \mathrm{G}_{r h}\left(\mathcal{O}_{U_{\mathbb{C}}}\right)\right) .
$$

Demonstration C'est une conséquence immédiate du Corollaire 4.13.

On note le résultat suivant qui ne sera pas utilisé dans ce paragraphe mais qui servira plus tard.

\section{Proposition 4.15 - On a un diagramme commutatif}

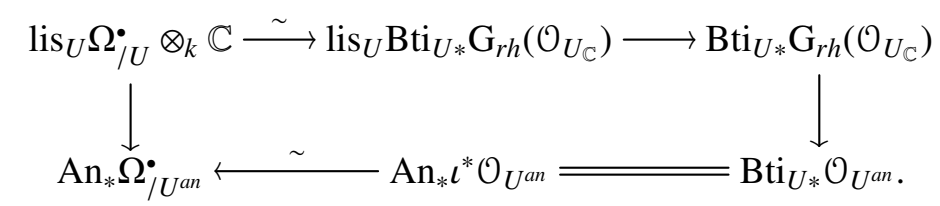

La flèche verticale à droite est induite par le morphisme (132) de la Proposition 4.8. 
Demonstration Les preuves du Théorème 4.11 et du Corollaire 4.12 montrent que, pour $M \in$ lisDM $\mathbf{M}^{\text {eff }}(U)$, on a un carré commutatif

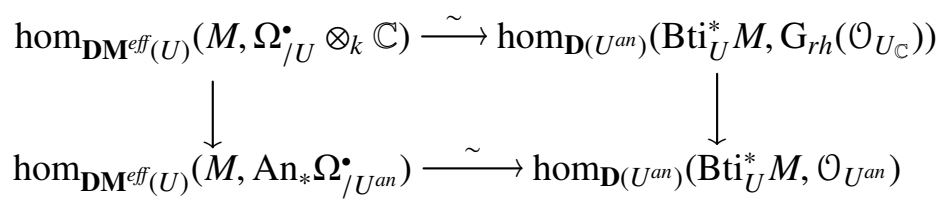

où la flèche verticale de droite est la composition de

$$
\begin{aligned}
\operatorname{hom}_{\mathbf{D}\left(U^{a n}\right)}\left(\mathrm{Bti}_{U}^{*} M, \mathrm{G}_{r h}\left(\mathcal{O}_{U_{\mathbb{C}}}\right)\right) & \simeq \operatorname{hom}{\operatorname{hod}\left(\mathcal{O}_{U}\right)}\left(\mathrm{RH}_{U}^{-1} \mathrm{H}_{0} \mathrm{Bti}_{U}^{*} M, \mathcal{O}_{U}\right) \\
& \longrightarrow \operatorname{hom} \operatorname{Shv}\left(U^{a n}\right)\left(\mathrm{H}_{0} \mathrm{Bti}_{U}^{*} M, \mathcal{O}_{U^{a n}}\right) \simeq \operatorname{hom}_{\mathbf{D}\left(U^{a n}\right)}\left(\mathrm{Bti}_{U^{*}}^{*} M, \mathcal{O}_{U^{a n}}\right) .
\end{aligned}
$$

C'est donc bien la flèche induite par (132). (Voir le début de la preuve de la Proposition 4.8 pour la construction de (132).)

LemMe 4.16 - Soient $U \subset \mathbb{A}_{k}^{1}$ un ouvert non vide et $N \in \mathbf{D M}^{\text {eff }}(U)$. Il existe un isomorphisme canonique (aux morphismes fantômes près) :

$$
\underset{\emptyset \neq V \subset U}{\operatorname{hocolim}_{\emptyset \neq U}}(F / V)^{*} \circ \operatorname{lis}_{V} \circ(V / U)^{*} N \simeq(F / U)^{*} N .
$$

Demonstration Les morphismes canoniques $(F / V)^{*} \circ \operatorname{lis}_{V} \circ(V / U)^{*} N \longrightarrow(F / U)^{*} N$ induisent un morphisme

$$
\underset{\emptyset \neq V \subset U}{\operatorname{hocolim}}(F / V)^{*} \circ \operatorname{lis}_{V} \circ(V / U)^{*} N \longrightarrow(F / U)^{*} N
$$

On montre que c'est un isomorphisme en vérifiant qu'il induit une bijection après application de $\operatorname{hom}_{\mathbf{D M}^{e f f}(F)}(M,-)$ avec $M$ compact. Quitte à rétrécir $U$, on peut supposer que $M=(F / U)^{*} M_{0}$ avec $M_{0}$ un objet compact de lisDM ${ }^{e f f}(U)$. En utilisant la variante avec transferts de [7, Prop. 1.A.1], on peut réécrire le morphisme qui nous intéresse comme la colimite des morphismes

$$
\operatorname{hom}_{\mathbf{D M}^{e f f}(V)}\left((V / U)^{*} M_{0}, \operatorname{lis}_{V} \circ(V / U)^{*} N\right) \longrightarrow \operatorname{hom}_{\mathbf{D M}^{e f f}(V)}\left((V / U)^{*} M_{0},(V / U)^{*} N\right) .
$$

Le résultat recherché est maintenant clair.

Proposition 4.17 — Il existe un isomorphisme canonique (aux morphismes fantômes près) dans $\mathbf{D M}^{e f f}(F)$ :

$$
\begin{aligned}
& \Omega_{/ F}^{\bullet} \otimes_{k} \mathbb{C} \simeq \operatorname{hocolim}_{\emptyset \neq U \subset \mathbb{A}_{k}^{1}}(F / U)^{*} \circ \operatorname{lis}_{U} \circ \operatorname{Bti}_{U *} \circ \mathrm{G}_{r h}\left(\mathcal{O}_{U_{\mathbb{C}}}\right) \\
& \simeq \underset{\emptyset \neq U \subset \mathbb{A}_{k}^{1}}{\operatorname{hocolim}}(F / U)^{*} \circ \operatorname{Bti}_{U *} \circ \mathrm{G}_{r h}\left(\mathcal{O}_{U_{\mathbb{C}}}\right) .
\end{aligned}
$$

Demonstration C'est la conjonction du Corollaire 4.14 et du Lemme 4.16.

On pose

$$
\mathcal{A}_{F \otimes_{k} \mathbb{C}}^{\mathrm{rs}}=\operatorname{colim}_{\emptyset \neq U \subset \mathbb{A}_{k}^{1}} \mathcal{A}_{U_{\mathbb{C}}}^{\mathrm{rs}}\left(U_{\mathbb{C}}\right) .
$$

Définition 4.18 - On définit un morphisme, dit d'évaluation, de $\left(F \otimes_{k} \mathbb{C}\right)$-algèbres

$$
\mathbf{P}^{e f f, \varpi}(F, \sigma) \otimes_{k} \mathbb{C} \longrightarrow \mathcal{A}_{F \otimes_{k} \mathbb{C}}^{\mathrm{rs}}
$$

comme étant le morphisme induit sur l’homologie en degré zéro par la composition de

$$
\begin{aligned}
& \operatorname{TgB}^{*}\left(\Omega_{/ F}^{\bullet}\right) \otimes_{k} \mathbb{C} \simeq \operatorname{TgB}^{*}\left(\Omega_{/ F}^{\bullet} \otimes_{k} \mathbb{C}\right) \simeq \underset{\emptyset \neq U \subset \mathbb{A}_{k}^{1}}{\operatorname{hocolim}} \operatorname{TgB}^{*}(F / U)^{*} \operatorname{lis}_{U} \operatorname{Bti}_{U *}\left(\mathrm{G}_{r h}\left(\mathcal{O}_{U_{\mathbb{C}}}\right)\right) \\
\simeq & \operatorname{hocolim}_{\emptyset \neq U \subset \mathbb{A}_{k}^{1}} \Psi_{U^{a n}} \operatorname{Bti}_{U}^{*} \operatorname{lis}_{U} \operatorname{Bti}_{U *}\left(\mathrm{G}_{r h}\left(\mathcal{O}_{U_{\mathbb{C}}}\right)\right) \stackrel{\delta}{\longrightarrow} \underset{\emptyset \neq U \subset \mathbb{A}_{k}^{1}}{\operatorname{hocolim}} \Psi_{U^{a n}} \mathrm{G}_{r h}\left(\mathcal{O}_{U_{\mathbb{C}}}\right) \simeq \mathcal{A}_{F \otimes_{k} \mathbb{C}}^{\mathrm{rs}} .
\end{aligned}
$$

(Le deuxième isomorphisme est déduit de la Proposition 4.17, le troisième isomorphisme est déduit $d u$ Lemme 2.17 et le morphisme $\delta$ s'obtient par adjonction.)

Proposition 4.19 - Le morphisme (155) est un morphisme de $\mathrm{C}_{f}^{0}\left(\pi_{1}^{\varpi}(\mathbb{C} \backslash \bar{k})\right.$, $\left.\mathbb{C}\right)$-comodules. 
Demonstration En effet, l'action de $\pi_{1}^{\varpi}(\mathbb{C} \backslash \bar{k})$ sur $\operatorname{TgB}^{*}\left(\Omega_{/ F}^{*}\right)$ est déduite, modulo les trois premiers isomorphismes dans (156), des actions canoniques de $\pi_{1}^{\varpi}\left(U^{a n}\right)$ sur les fibres tangentielles en $0 \in \mathbb{C}$ des ind-systèmes locaux $\mathrm{Bti}_{U}^{*} \operatorname{lis}_{U} \mathrm{Bti}_{U *}\left(\mathrm{G}_{r h}\left(\mathcal{O}_{U_{\mathbb{C}}}\right)\right)$. Il suffit donc de montrer que l'avantdernier morphisme dans (156) est équivariant. Ceci est clair : il s'agit de la colimite des morphismes induits sur les fibres tangentielles en $0 \in \mathbb{C}$ par les morphismes de ind-systèmes locaux $\mathrm{Bti}_{U}^{*} \operatorname{lis}_{U} \mathrm{Bti}_{U *}\left(\mathrm{G}_{r h}\left(\mathcal{O}_{U_{\mathrm{C}}}\right)\right) \longrightarrow \mathrm{G}_{r h}\left(\mathcal{O}_{U_{\mathrm{C}}}\right)$.

Remarque 4.20 - Le morphisme (155) est un morphisme de $\mathcal{D}(F) \otimes_{k} \mathbb{C}=\left(F \otimes_{k} \mathbb{C}\right)\left[\partial_{\varpi}\right]$ modules En effet, le Théorème 4.11 entraîne que le morphisme (152) du Corollaire 4.14 commute aux opérateurs $\partial_{\varpi}$.

THÉORÈME 4.21 - La composition de

$$
\mathbf{Q}^{e f f, \varpi}(F, \sigma) \longrightarrow \mathbf{P}^{e f f, \varpi}(F, \sigma) \longrightarrow \mathcal{A}_{F \otimes_{k} \mathbb{C}}^{\mathrm{rs}}
$$

envoie la classe d'une série $G \in \mathcal{O}_{k-a l g}^{\dagger}\left(\overline{\mathbb{D}}^{\infty}\right)$ (cf. le Corollaire 3.23) sur la série de Laurent $\int_{[0,1]^{\infty}} G$.

Remarque 4.22 - Il est également possible de déterminer les images de $\varpi^{1 / r}, \ln \varpi \in \mathbf{P}^{\varpi}(F, \sigma)$ par le morphisme (155) : ce sont $\varpi^{1 / r}, \ln \varpi \in \mathcal{A}_{F \otimes_{k} \mathbb{C}}^{\text {rs }}$. Joint au Théorème 4.21, ceci fournit une description complète du morphisme d'évaluation $\mathbf{P}^{\varpi}(F, \sigma) \longrightarrow \mathcal{A}_{F \otimes_{k} \mathbb{C}}^{\text {rs }}$. Toutefois, nous n'aurons pas besoin de ce résultat plus complet.

La preuve du Théorème 4.21 sera donnée dans les paragraphes suivants : il faudra alors démêler les définitions, ce qui ne sera pas une tâche facile.

THÉORÈME 4.23 - Il existe un carré commutatif

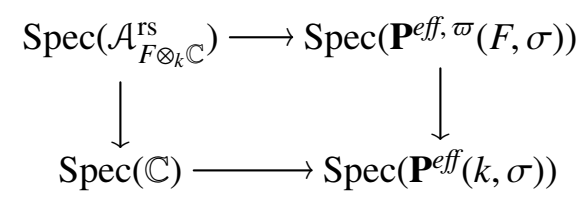

où les flèches horizontales sont données par les morphismes d'évaluation (voir (109) et la Définition 4.18). Le morphisme induit (cf. (113))

$$
\operatorname{Spec}\left(\mathcal{A}_{F \otimes_{k} \mathbb{C}}^{\mathrm{rs}}\right) \longrightarrow \operatorname{Spec}\left(\mathbf{R}_{/ k}^{\varpi}(F, \sigma)\right)
$$

est un morphisme $\pi_{1}^{\varpi}(\mathbb{C} \backslash \bar{k})$-équivariant de $\left(F \otimes_{k} \mathbb{C}\right)$-schémas. De plus, l'action de la complétion pro-C-algébrique de $\pi_{1}^{\Phi}(\mathbb{C} \backslash \bar{k})$ est transitive sur la source et le but de (158); elle est même simplement transitive sur la source. (Bien entendu, la notion de transitivité considérée ici est relative au schéma de base $\operatorname{Spec}\left(F \otimes_{k} \mathbb{C}\right)$.)

Demonstration Pour vérifier que le carré de l'énoncé commute, on remarque que le morphisme canonique $\mathbf{P}^{e f f}(k, \sigma) \longrightarrow \mathbf{P}^{e f f,}$, $(F, \sigma)$ se factorise par $\mathbf{Q}^{e f f}{ }^{\varpi}(F, \sigma)$. Le résultat recherché est alors une conséquence du Théorème 4.21 .

Le reste de l'énoncé s'obtient en combinant le Théorème 3.10, la Proposition 3.26, le Corollaire 4.7 et le fait que la flèche horizontale supérieure dans le carré de l'énoncé est équivariante sous l'action de $\pi_{1}^{\varpi \pi}(\mathbb{C} \backslash \bar{k})$ d'après la Proposition 4.19.

Corollaire 4.24 - Le morphisme de $\left(F \otimes_{k} \mathbb{C}\right)$-algèbres $\mathbf{R}_{/ k}^{\omega}(F, \sigma) \longrightarrow \mathcal{A}_{F \otimes_{k} \mathbb{C}}^{\mathrm{rs}}$ fourni par le Théorème 4.23 est injectif. De plus, ce morphisme envoie $[G] \otimes \lambda\left(\right.$ avec $G \in \mathcal{O}_{k-a l g}^{\dagger}\left(\overline{\mathbb{D}}^{\infty}\right)$ et $\lambda \in \mathbb{C}$ ) $\operatorname{sur} \lambda \cdot \int_{[0,1]^{\infty}} G$.

Demonstration La première assertion découle aussitôt du Théorème 4.23. La seconde assertion découle de la détermination du morphisme d'évaluation, i.e., du Théorème 4.21.

THÉORÈme 4.25 - Le noyau de (9) est le sous-F-espace vectoriel engendré par les éléments de la forme

$$
\frac{\partial G}{\partial z_{i}}-\left.G\right|_{z_{i}=1}+\left.G\right|_{z_{i}=0} \quad \text { et } \quad f \cdot L
$$


avec $G, L \in \mathcal{O}_{k-a l g}^{\dagger}\left(\overline{\mathbb{D}}^{\infty}\right), i \in \mathbb{N} \backslash\{0\}$ et $f \in \mathcal{O}_{k-a l g}\left(\overline{\mathbb{D}}^{\infty}\right)$ vérifiant $\int_{[0,1]^{\infty}} f=0$, et telle que $f$ et $L$ ne dépendent pas simultanément d'une même variable complexe.

Demonstration Ceci est maintenant une conséquence du Théorème 3.27.

Démonstration du Théorème 1.8 Soit $F \in \mathcal{O}_{\mathbb{C}-a l g}^{\dagger}\left(\overline{\mathbb{D}}^{\infty}\right)$ telle que $\int_{[0,1]^{\infty}} F=0$. Puisque $F$ est algébrique, on peut trouver un sous-corps $k \subset \mathbb{C}$, de type fini sur $\mathbb{Q}$, tel que $F \in \mathcal{O}_{k-\text { alg }}^{\dagger}\left(\overline{\mathbb{D}}^{\infty}\right)$. On conclut en lui appliquant le Théorème 4.25 .

4.4. Le morphisme d'évaluation, première partie. - Dans ce paragraphe et les deux qui suivront, nous démontrerons le Théorème 4.21 qui a joué un rôle essentiel dans la preuve du Théorème 1.8 .

On considère la situation suivante. Soit $V \subset \mathbb{A}_{k}^{1}$ un voisinage Zariski de $0 \in \mathbb{A}_{k}^{1}$ et notons $U=V \backslash\{0\}$. On suppose donné un morphisme étale $v: Y \longrightarrow \mathbb{A}_{V}^{n}$ et on forme le diagramme commutatif à carrés cartésiens

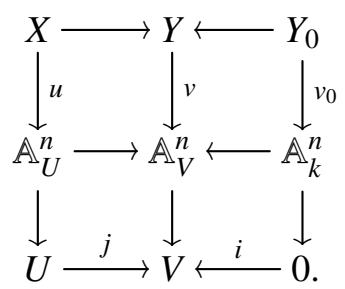

On se donne aussi un morphisme $\overline{\mathbb{D}}^{n} \longrightarrow Y_{0}^{\text {an }}$ rendant le triangle

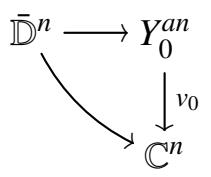

commutatif. On en déduit des morphismes étales de pro-schémas :

$$
b_{0}: \overline{\mathbb{D}}_{\text {ét }}^{n} \longrightarrow Y_{0} \quad \text { et } \quad a: \overline{\mathbb{D}}_{\text {ét }}^{\dagger, n} \longrightarrow X .
$$

(Pour définir $a$, il suffit de spécifier un morphisme de pro-schémas formels $\overline{\mathbb{D}}_{\text {ét }}^{n}[[\varpi]] \longrightarrow Y / /(\varpi)$. Un tel morphisme est uniquement déterminé par le morphisme pro-étale $\overline{\mathbb{D}}_{\text {ét }}^{n} \longrightarrow Y_{0}$ grâce à $[\mathbf{1 5}$, Chap. IV, Th. 18.1.2].) On note $\partial X \subset X, \partial Y \subset Y$ et $\partial Y_{0} \subset Y_{0}$ les images inverses du bord de $\mathbb{A}_{k}^{n}$, i.e., les diviseurs à croisements normaux définis par l'équation $\prod_{i=1}^{n} z_{i}\left(z_{i}-1\right)=0$. (On prend $\mathbb{A}_{k}^{n}=\operatorname{Spec}\left(k\left[z_{1}, \cdots, z_{n}\right]\right)$.) Enfin, on fixe une forme différentielle relative $\omega \in \Omega_{/ U}^{n}(X)$. On peut écrire :

$$
\omega=G \cdot \mathrm{d} z_{1} \wedge \cdots \wedge \mathrm{d} z_{n}
$$

pour une certaine fonction régulière $G \in \mathcal{O}(X)$.

On considère le motif $M=\mathbb{Q}_{t r}(X / \partial X) \in \mathbf{D M}^{e f f}(U)$. (Rappelons que la notation $\mathbb{Q}_{t r}(W / Z)$, pour $W$ une variété algébrique, analytique rigide ou complexe, et $Z \subset W$ un sous-ensemble localement fermé, signifie le préfaisceau avec transferts coker $\left\{\mathbb{Q}_{t r}(Z) \rightarrow \mathbb{Q}_{t r}(W)\right\}$.)

D'une part, le morphisme $a: \overline{\mathbb{D}}_{\text {êt }}^{\dagger, n} \longrightarrow X$ définit une classe d'homologie

$$
\gamma \in \mathrm{H}_{n} \mathrm{C}\left(M\left(\overline{\mathbb{D}}_{\text {ett }}^{\dagger}\right)\right) \simeq \mathrm{H}_{n} \mathrm{Bti}^{*} \chi(F / U)^{*} M .
$$

(Pour l'isomorphisme ci-dessus, on utilise le Corollaire 2.27.) D'autre part, la forme différentielle $\omega$ définit un morphisme $\omega: M[-n] \longrightarrow \Omega_{/ U}^{\cdot}$ dans $\mathbf{D M}^{e f f}(U)$. (On utilise que $\omega \in \Omega_{/ U}^{n}(X)$ s'annule sur le bord de $X$ pour une raison de dimension.) On peut donc former la composition de

$$
\langle a, \omega\rangle: \mathbb{Q} \stackrel{\gamma}{\longrightarrow} \operatorname{Bti}^{*} \chi(F / U)^{*} M[-n] \stackrel{\omega}{\longrightarrow} \operatorname{Bti}^{*} \chi \Omega_{/ F}^{\bullet} .
$$

Ceci définit un élément $\langle a, \omega\rangle \in \mathbf{Q}^{e f f, \varpi}(F, \sigma)$ (voir la Proposition 3.22). Le résultat suivant est une conséquence directe de la preuve du Corollaire 3.23. 
Lemme 4.26 - L'élement $\langle a, \omega\rangle$ est donné par la classe de $G \circ a \in \mathcal{O}_{k-a l g}^{\dagger}\left(\overline{\mathbb{D}}^{n}\right)$ modulo l'identification du Corollaire 3.23. De plus, tout élément de $\mathbf{Q}^{\text {eff, }}(F, \sigma)$ s'écrit sous la forme $\langle a, \omega\rangle$ pour a et $\omega$ bien choisis.

Ainsi, pour montrer le Théorème 4.21, il suffit de vérifier que l'image de $\langle a, \omega\rangle$ par la composition de (157) est donnée par $\int_{[0,1]^{n}} G \circ a$. À ce stade, il est utile de rappeler que la composition de (157) est donnée, après passage à l'homologie en degré zéro, par la composition de

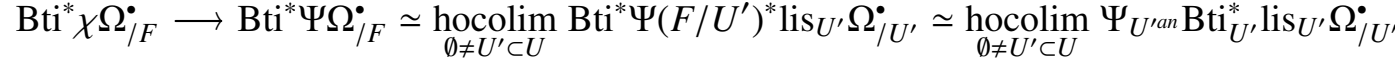

$$
\begin{aligned}
& \longrightarrow \underset{\emptyset \neq U^{\prime} \subset U}{\operatorname{hocolim}} \Psi_{U^{\prime a n}} \mathrm{Bti}_{U^{\prime}}^{*} \operatorname{lis}_{U^{\prime}} \mathrm{Bti}_{U^{\prime} *} \mathrm{G}_{r h}\left(\mathcal{O}_{U_{\mathbb{C}}^{\prime}}\right) \stackrel{\delta}{\longrightarrow} \underset{\emptyset \neq c o l i m}{\operatorname{hocol} \subset U} \Psi_{U^{\prime a n}} \mathrm{G}_{r h}\left(\mathcal{O}_{U_{\mathbb{C}}^{\prime}}\right) \simeq \mathcal{A}_{F \otimes_{k} \mathbb{C}}^{\mathrm{rs}}
\end{aligned}
$$

(Dorénavant et comme ci-dessus, nous noterons simplement $\Psi$ le foncteur « motif proche » de la Définition 2.6; auparavant, ce foncteur était désigné par $\Psi_{\varpi}$.) Quitte à rétrécir $U$, on peut supposer que le $U$-motif $M$ devient fortement dualisable dans $\mathbf{D M}(U)$. Dans ce cas, le morphisme $\omega \in$ $\operatorname{hom}_{\mathbf{D M}^{e f f}(U)}\left(M[-n], \Omega_{/ U}^{\cdot}\right)$ provient d'un unique morphisme $\tilde{\omega} \in \operatorname{hom}_{\mathbf{D M}^{e f f}(U)}\left(M[-n], \operatorname{lis}_{U} \Omega_{/ U}^{\cdot}\right)$. Il est alors clair que l'image de $\langle a, \omega\rangle$ par la composition de (157) correspond à la composition de

$$
\begin{aligned}
& \mathbb{Q} \stackrel{\gamma}{\longrightarrow} \operatorname{Bti}^{*} \chi(F / U)^{*} M[-n] \stackrel{\tilde{\omega}}{\longrightarrow} \operatorname{Bti}^{*} \chi(F / U)^{*} \operatorname{lis}_{U} \Omega_{/ U}^{\cdot} \longrightarrow \operatorname{Bti}^{*} \Psi(F / U)^{*} \operatorname{lis}_{U} \Omega_{/ U}^{\bullet} \\
& \simeq \Psi_{U^{a n}} \mathrm{Bti}_{U}^{*} \operatorname{lis}_{U} \Omega_{/ U}^{\cdot} \longrightarrow \Psi_{U^{a n}} \mathrm{Bti}_{U}^{*} \operatorname{lis}_{U} \operatorname{Bti}_{U *} \mathrm{G}_{r h}\left(\mathcal{O}_{U_{\mathbb{C}}}\right) \longrightarrow \Psi_{U^{a n}} \mathrm{G}_{r h}\left(\mathcal{O}_{U_{\mathbb{C}}}\right) \simeq \mathcal{A}_{U_{\mathbb{C}}}^{\mathrm{rs}}\left(U_{\mathbb{C}}\right) \text {. }
\end{aligned}
$$

Évidemment, la composition de (161) est égale à celle de

$$
\begin{array}{r}
\mathbb{Q} \stackrel{\gamma}{\longrightarrow} \mathrm{Bti}^{*} \chi(F / U)^{*} M[-n] \longrightarrow \mathrm{Bti}^{*} \Psi(F / U)^{*} M[-n] \simeq \Psi_{U^{a n}} \mathrm{Bti}_{U}^{*} M[-n] \stackrel{\tilde{\omega}}{\longrightarrow} \\
\Psi_{U^{a n}} \mathrm{Bti}_{U}^{*} \operatorname{lis}_{U} \Omega_{/ U}^{\cdot} \longrightarrow \Psi_{U^{a n}} \mathrm{Bti}_{U}^{*} \operatorname{lis}_{U} \mathrm{Bti}_{U *} \mathrm{G}_{r h}\left(\mathcal{O}_{U_{\mathrm{C}}}\right) \longrightarrow \Psi_{U^{a n}} \mathrm{G}_{r h}\left(\mathcal{O}_{U_{\mathrm{C}}}\right) \simeq \mathcal{A}_{U_{\mathrm{C}}}^{\mathrm{rs}}\left(U_{\mathbb{C}}\right) .
\end{array}
$$

On a donc établi le fait suivant.

Lemme 4.27 - Pour démontrer le Théorème 4.21, il suffit de vérifier que la composition de (162) envoie $1 \in \mathbb{Q}$ sur $\int_{[0,1]^{n}} G \circ a$.

Pour continuer, nous aurons besoin d'une courte digression. Dans [2, Chap. 3] nous avons considéré les foncteurs $\chi_{U}=i^{*} j_{*}, \Psi_{U}: \mathbf{D A}^{\text {ét }}(U) \longrightarrow \mathbf{D A}^{\text {êt }}(k)$ et nous avons montré que ces foncteurs jouissaient des mêmes propriétés formelles que leurs analogues classiques (par exemple en cohomologie étale). Vu les équivalences de catégories $\mathbf{D} \mathbf{A}^{\text {ét }}(U) \simeq \mathbf{D M}(U)$ et $\mathbf{D A}^{\text {ét }}(k) \simeq \mathbf{D M}(k)$, on déduit deux foncteurs $\chi_{U}=i^{*} j_{*}, \Psi_{U}: \mathbf{D M}(U) \longrightarrow \mathbf{D M}(k)$. Toutefois, pour les motifs effectifs, on ne dispose pas du formalisme des opérations de Grothendieck [1, Chap. 1] et on ignore si les définitions de [2, Chap. 3] fournissent des foncteurs raisonnables. Nous sommes donc amenés à définir les foncteurs $\chi_{U}, \Psi_{U}: \mathbf{D M}^{\text {eff }}(U) \longrightarrow \mathbf{D M}^{\text {eff }}(k)$ en passant par les motifs stables, i.e., en posant

$$
\chi_{U}=\mathrm{Ev}_{0} \circ i^{*} \circ j_{*} \circ \operatorname{Sus}_{T}^{0} \quad \text { et } \quad \Psi_{U}=\mathrm{Ev}_{0} \circ \Psi_{U} \circ \operatorname{Sus}_{T}^{0} .
$$

(Rappelons que $\operatorname{Sus}_{T}^{0}: \mathbf{D M}^{e f f}(-) \longrightarrow \mathbf{D M}(-)$ est le foncteur de suspension infinie et $\mathrm{Ev}_{0}$ est son adjoint à droite.) On a le résultat suivant.

Proposition 4.28 - Il existe des isomorphismes canoniques

$$
\chi(F / U)^{*} \simeq \chi_{U} \quad \text { et } \quad \Psi(F / U)^{*} \simeq \Psi_{U}
$$

entre foncteurs de $\mathbf{D} \mathbf{M}^{\text {eff }}(U)$ dans $\mathbf{D M}^{\text {eff }}(k)$. De plus, le carré suivant

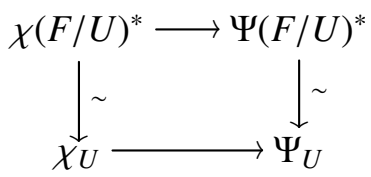

commute.

Demonstration L'énoncé analogue pour les catégories DA(-) découle immédiatement de [7, Scholie 1.3.26, (2)]. 
Montrons que les foncteurs $\chi, \chi_{U}, \Psi$ et $\Psi_{U}$ commutent aux foncteurs aét $: \mathbf{D A}(-) \longrightarrow \mathbf{D A}^{\text {ét }}(-)$. Les foncteurs aét commutent aux quatre opérations de Grothendieck associées à un morphisme de schémas; c'est une conséquence facile de [3, Th. 3.4 et 3.7] et du fait que ces opérations commutent aux sommes infinies (pour $\mathbf{D} \mathbf{A}^{\text {ét }}(-)$ c'est vrai puisqu'on travaille à coefficients rationnels). Ceci permet de conclure pour $\chi_{U}$ et $\Psi_{U}$. Le foncteur $\chi$ est la composition de (38) et le foncteur $\Psi$ est la composition de (23). Or, les trois premiers foncteurs dans ces compositions commutent aux foncteurs aét et les autres foncteurs sont des opérations de Grothendieck. La commutation recherchée est donc aussi vraie pour $\chi$ et $\Psi$. Puisque les foncteurs aét sont des foncteurs de localisation, on déduit de ce qui précède la variante de la proposition pour les catégories $\mathbf{D A}{ }^{{ }^{\mathrm{e}}}(-)$. En utilisant les équivalences de Cisinski-Déglise $\mathbf{D} \mathbf{A}^{\text {ét }}(-) \simeq \mathbf{D M}(-)$, on a aussi la variante de la proposition pour les catégories $\mathbf{D M}(-)$.

Pour terminer, il reste à prouver que les transformations naturelles

$$
\chi \longrightarrow \mathrm{Ev}_{0} \circ \chi \circ \operatorname{Sus}_{T}^{0} \quad \text { et } \quad \Psi \longrightarrow \mathrm{Ev}_{0} \circ \Psi \circ \operatorname{Sus}_{T}^{0}
$$

sont inversibles. Puisque les trois premiers foncteurs dans (23) et (38) commutent aux foncteurs de suspension infinie $\operatorname{Sus}_{T}^{0}$, on se ramène à montrer que les transformations naturelles

$$
q_{*} \longrightarrow \mathrm{Ev}_{0} \circ q_{*} \circ \operatorname{Sus}_{T}^{0} \quad \text { et } \quad 1^{*} \longrightarrow \mathrm{Ev}_{0} \circ 1^{*} \circ \operatorname{Sus}_{T}^{0}
$$

sont inversibles après restriction à la sous-catégorie $\mathbf{q u D M}^{\text {eff }}(k)$. Ceci découle des isomorphismes de commutation $\mathrm{Ev}_{0} \circ q_{*} \simeq q_{*} \circ \operatorname{Ev}_{0}$ et $1^{*} \circ \operatorname{Sus}_{T}^{0} \simeq \operatorname{Sus}_{T}^{0} \circ 1^{*}$ et du «cancellation theorem » dans $\mathbf{q u D M}^{\text {eff }}(k)$ et $\mathbf{D M}^{\text {eff }}(k)$ respectivement (voir le Lemme 2.5).

Proposition 4.29 - Il existe des transformations naturelles

$$
\mathrm{Bti}^{*} \chi_{U} \longrightarrow \chi_{U^{a n}} \mathrm{Bti}_{U}^{*} \quad \text { et } \quad \mathrm{Bti}^{*} \Psi_{U} \longrightarrow \Psi_{U^{a n}} \mathrm{Bti}_{U}^{*}
$$

entre foncteurs de $\mathbf{D} \mathbf{M}^{\text {eff }}(U)$ dans $\mathbf{D}(\mathbb{Q})$; la seconde étant inversible sur les objets constructibles de $\mathbf{D M}^{e f f}(U)$. De plus, le carré suivant

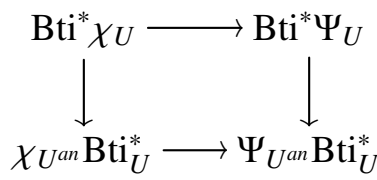

commute.

Demonstration Par construction, on dispose des transformations naturelles

$$
\operatorname{Sus}_{T}^{0} \circ \chi_{U} \longrightarrow \chi_{U} \circ \operatorname{Sus}_{T}^{0} \quad \text { et } \quad \operatorname{Sus}_{T}^{0} \circ \Psi_{U} \longrightarrow \Psi_{U} \circ \operatorname{Sus}_{T}^{0} .
$$

En utilisant l'identification $\Psi(F / U)^{*} \simeq \Psi_{U}$ (dans les cas effectif et stable) et la Remarque 2.7, on déduit aussitôt que $\Psi_{U}$ commute aux foncteurs de suspension infinie. Autrement dit, la seconde transformation naturelle dans (165) est inversible.

En appliquant le foncteur Bti* et en utilisant l'isomorphisme $\mathrm{Bti}^{*} \simeq \mathrm{Bti}^{*} \circ \mathrm{Sus}_{T}^{0}$, on déduit des transformations naturelles

$$
\mathrm{Bti}^{*} \circ \chi_{U} \longrightarrow \mathrm{Bti}^{*} \circ \chi_{U} \circ \mathrm{Sus}_{T}^{0} \quad \text { et } \quad \mathrm{Bti}^{*} \circ \Psi_{U} \longrightarrow \mathrm{Bti}^{*} \circ \Psi_{U} \circ \operatorname{Sus}_{T}^{0} .
$$

La seconde est encore inversible. D'après [3], on dispose dans le contexte stable de morphismes de comparaison

$$
\mathrm{Bti}^{*} \circ \chi_{U} \longrightarrow \chi_{U} \circ \mathrm{Bti}_{U}^{*} \quad \text { et } \quad \mathrm{Bti}^{*} \circ \Psi_{U} \longrightarrow \Psi_{U} \circ \mathrm{Bti}_{U}^{*},
$$

qui sont inversibles sur les objets constructibles. En composant les transformations naturelles de (166) avec celles de (167), et en utilisant à nouveau l'isomorphisme $\mathrm{Bti}_{U}^{*} \simeq \mathrm{Bti}_{U}^{*} \circ \mathrm{Sus}_{T}^{0}$, on obtient les transformations naturelles recherchées. La seconde est alors inversible sur les objets constructibles de $\mathbf{D} \mathbf{M}^{e f f}(U)$. 
L'isomorphisme $\mathrm{Bti}^{*} \Psi(F / U)^{*} \simeq \Psi_{U^{a n}} \mathrm{Bti}_{U}^{*}$ utilisé dans (162) est la composition des isomorphismes $\Psi(F / U)^{*} \simeq \Psi_{U}$ et $\mathrm{Bti}^{*} \Psi_{U} \simeq \Psi_{U^{a n} \mathrm{Bti}_{U}^{*}}$ des Propositions 4.28 et 4.29. Il s'ensuit que la composition de (162) coïncide avec la composition de

$$
\begin{aligned}
& \mathbb{Q} \stackrel{\gamma}{\longrightarrow} \operatorname{Bti}^{*} \chi(F / U)^{*} M[-n] \simeq \mathrm{Bti}^{*} \chi_{U} M[-n] \longrightarrow \chi_{U^{a n}} \mathrm{Bti}_{U}^{*} M[-n] \stackrel{\tilde{\omega}}{\longrightarrow} \chi_{U^{a n}} \mathrm{Bti}_{U}^{*} \operatorname{lis}_{U} \Omega_{/ U}^{\cdot} \\
& \longrightarrow \chi_{U^{a n}} \mathrm{Bti}_{U}^{*} \operatorname{lis}_{U} \mathrm{Bti}_{U *} \mathrm{G}_{r h}\left(\mathcal{O}_{U_{\mathrm{C}}}\right) \longrightarrow \chi_{U^{a n}} \mathrm{G}_{r h}\left(\mathcal{O}_{U_{\mathrm{C}}}\right) \longrightarrow \Psi_{U^{a n}} \mathrm{G}_{r h}\left(\mathcal{O}_{U_{\mathbb{C}}}\right) \simeq \mathcal{A}_{U_{\mathbb{C}}}^{\mathrm{rs}}\left(U_{\mathbb{C}}\right) .
\end{aligned}
$$

On a donc établi le fait suivant.

Lemme 4.30 - Pour démontrer le Théorème 4.21, il suffit de vérifier que la composition de (168) envoie $1 \in \mathbb{Q}$ sur $\int_{[0,1]^{n}} G \circ a$.

4.5. Le morphisme d'évaluation, deuxième partie. - Le but de ce paragraphe est de simplifier la composition des trois premiers morphismes dans (168), i.e., la composition de

$$
\mathbb{Q}[n] \stackrel{\gamma}{\longrightarrow} \mathrm{Bti}^{*} \chi(F / U)^{*} M \simeq \mathrm{Bti}^{*} \chi_{U} M \longrightarrow \chi_{U^{a n} \mathrm{Bti}_{U}^{*} M .}
$$

Pour ce faire, nous devons d'abord expliciter l'isomorphisme $\chi(F / U)^{*} \simeq \chi_{U}$. On reprendra la situation considérée au §4.4 à partir de la seconde moitié du paragraphe. Pour l'instant, seuls les schémas $U$ et $V$ du $\$ 4.4$ seront utilisés. (Ainsi, dans la première moitié du paragraphe, la lettre $X$ désignera des schémas ou des variétés analytiques rigides qui n'ont rien à voir avec le $U$-schéma $X \mathrm{du} \S 4.4$.)

Dans le contexte effectif, il y a lieu de considérer le foncteur $\chi_{U}^{\text {bête }}: \mathbf{D M}^{e f f}(U) \longrightarrow \mathbf{D M}^{\text {eff }}(k)$ donné par $\chi_{U}^{\text {bête }}=i^{*} j_{*}$. On dispose d'une transformation naturelle évidente $\chi_{U}^{\text {bête }} \longrightarrow \chi_{U}$; elle est donnée par la composition de

$$
i^{*} \circ j_{*} \longrightarrow i^{*} \circ j_{*} \circ \operatorname{Ev}_{0} \circ \operatorname{Sus}_{T}^{0} \simeq i^{*} \circ \operatorname{Ev}_{0} \circ j_{*} \circ \operatorname{Sus}_{T}^{0} \longrightarrow \operatorname{Ev}_{0} \circ i^{*} \circ j_{*} \circ \operatorname{Sus}_{T}^{0} .
$$

Nous ignorons si cette transformation naturelle est inversible.

Soit $L$ un complexe de préfaisceaux avec transferts sur $\mathrm{Sm} / U$. On fixe un remplacement ( $\mathbb{A}^{1}$, ét)fibrant $L \longrightarrow L^{\prime}$, i.e., une équivalence ( $\mathbb{A}^{1}$, ét)-locale telle que $L^{\prime}$ est ( $\mathbb{A}^{1}$, ét)-fibrant. On fixe aussi un quasi-isomorphisme de complexes de préfaisceaux avec transferts $L^{\prime \prime} \longrightarrow j_{*} L^{\prime}$ avec $L^{\prime \prime}$ projectivement cofibrant. Pour un $k$-schéma affine et lisse $X$, on note $Q^{f o r}(X)$ le schéma formel $X[[\varpi]]$ et $Q_{\text {ét }}^{f o r}(X)$ le pro-schéma des voisinages étales de $X \simeq X[\varpi] /(\varpi)$ dans $X[\varpi]$. Si $X=\operatorname{Spec}(A)$, alors $\mathcal{O}\left(Q_{\text {êt }}^{f o r}(X)\right)$ est la sous-algèbre de $A[[\varpi]]$ formée des éléments qui sont algébriques sur le corps des fractions de $A[\varpi]$ (cf. [4, Prop. 2.102] pour un résultat similaire); c'est également l'union filtrante des sous- $A[\varpi]$-algèbres étales de $A[[\varpi]]$. Aussi, on a $Q^{f o r}(X)\left[\varpi^{-1}\right]=Q^{\text {rig }}(X)$ et $Q_{\mathrm{ett}}^{f o r}(X)\left[\varpi^{-1}\right]=Q_{\mathrm{et}}^{r i g}(X)$. On dispose de morphismes de complexes :

$$
L^{\prime \prime}\left(Q_{\mathrm{et}}^{f o r}(X)\right) \longrightarrow j_{*} L^{\prime}\left(Q_{\mathrm{e} \mathrm{t}}^{f o r}(X)\right)=L^{\prime}\left(Q_{\mathrm{e} \mathrm{t}}^{r i g}(X)\right) \quad \text { et } \quad L^{\prime \prime}\left(Q_{\mathrm{e} \mathrm{t}}^{f o r}(X)\right) \longrightarrow i^{*} L^{\prime \prime}(X)
$$

naturels en $X \in \mathrm{Sm} / k$. Le premier est un quasi-isomorphisme et le second est déduit de l'identification $Q_{\mathrm{et}}^{f o r}(X) \times_{V} 0 \simeq X$. Il s'ensuit un morphisme dans $\mathbf{D M}^{\text {eff }}(k)$ :

$$
\mathfrak{Q}_{*}\left(L^{\prime}\right) \longrightarrow i^{*} L^{\prime \prime}=\chi_{U}^{\text {bête }} L
$$

(cf. le Théorème 2.24). On a le résultat suivant.

Proposition 4.31 - Les compositions de

$$
\mathfrak{Q}_{*}(L) \simeq \chi(F / U)^{*} L \simeq \chi_{U} L \quad \text { et de } \quad \mathfrak{Q}_{*}(L) \longrightarrow \mathfrak{Q}_{*}\left(L^{\prime}\right) \longrightarrow \chi_{U}^{\text {bête }} L \longrightarrow \chi_{U} L
$$

sont égales dans $\mathbf{D M}^{\text {eff }}(k)$.

Demonstration On divise la preuve en trois étapes. La première est une réduction à la variante stable et sans transferts. Dans la seconde, on rappelle des résultats de [7]. Dans la troisième, on donne une description concrète de l'isomorphisme $\chi(F / U)^{*} \simeq \chi_{U}$ et on termine la preuve.

Étape $A$ : On ne restreint pas la généralité en supposant que $L$ est projectivement cofibrant. On note $\mathbf{L}=\operatorname{Sus}_{T}^{0}(L)$ et on fixe un remplacement projectivement stablement $\left(\mathbb{A}^{1}\right.$, ét)-fibrant $\mathbf{L} \longrightarrow \mathbf{L}^{\prime}$. 
On se donne aussi un morphisme $\mathbf{L}^{\prime \prime} \longrightarrow j_{*} \mathbf{L}^{\prime}$ qui est un quasi-isomorphisme niveau par niveau et tel que $\mathbf{L}^{\prime \prime}$ est projectivement cofibrant. Comme avant, on peut former le morphisme $\mathfrak{Q}_{*}\left(\mathbf{L}^{\prime}\right) \longrightarrow i^{*} \mathbf{L}^{\prime \prime}=\chi_{U}(\mathbf{L})$ dans $\mathbf{D M}(k)$. Il est alors immédiat que le diagramme suivant est commutatif

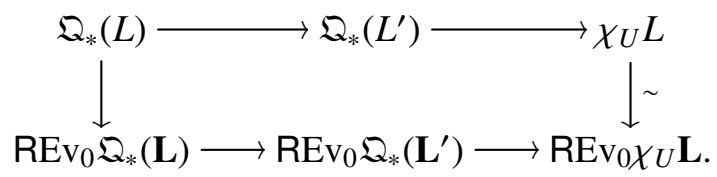

(Pour démontrer cela, il faut choisir les remplacements fibrants et cofibrants d'une manière compatible.) Il est donc suffisant de prouver la variante stable de la proposition. Autrement dit, il suffit de montrer que les compositions de

$$
\mathfrak{Q}_{*}(\mathbf{L}) \simeq \chi(F / U)^{*} \mathbf{L} \simeq \chi_{U} \mathbf{L} \quad \text { et de } \quad \mathfrak{Q}_{*}(\mathbf{L}) \longrightarrow \mathfrak{Q}_{*}\left(\mathbf{L}^{\prime}\right) \longrightarrow \chi_{U} \mathbf{L}
$$

sont égales dans $\mathbf{D M}(k)$.

Par ailleurs, vu les équivalences de catégories $\mathbf{D A}^{\mathrm{e}}{ }^{(-)} \simeq \mathbf{D M}(-)$, il suffit de démontrer la version sans transferts de la proposition pour le $T$-spectre $\mathrm{o}_{t r} \mathbf{L}$. (Rappelons que $\mathrm{o}_{t r}$ préserve les équivalences $\left(\mathbb{A}^{1}\right.$, ét)-locales stables d'après $[4$, Lem. 2.112].) On peut même travailler avec la topologie Nisnevich au lieu de la topologie étale. Ceci permettra d'invoquer plus facilement certains résultats de [7, Chap. 1]. Ainsi, dans la suite, $\mathbf{L}$ sera un $T$-spectre de préfaisceaux (sans transferts), $\mathbf{L} \longrightarrow \mathbf{L}^{\prime}$ un remplacement projectivement stablement (A ${ }^{1}$, Nis)-fibrant et $\mathbf{L}^{\prime \prime} \longrightarrow j_{*} \mathbf{L}^{\prime}$ un quasiisomorphisme niveau par niveau avec $\mathbf{L}^{\prime \prime}$ projectivement cofibrant. Toutefois, on prendra garde que le Théorème 2.32 n'est plus applicable et donc qu'on ne sait plus que le morphisme évident $\mathfrak{Q}_{*}(\mathbf{L}) \longrightarrow \chi(F / U)^{*} \mathbf{L}$ est un isomorphisme dans $\mathbf{D A}(k)$.

Étape $B$ : Dans cette étape, on reprend quelques notations et constructions de [7, §1.3.4]. On rappelle que $K=k((\varpi))$ et $K^{\circ}=k[[\varpi]]$. Si $A$ est une $K$-algèbre affinoïde, on note $A^{\circ}$ (resp. $\left.A^{\vee}\right)$ le sous-anneau de $A$ (resp. l'idéal de $A^{\circ}$ ) formé des éléments de $A$ dont la norme infinie est plus petite ou égale à 1 (resp. strictement plus petite que 1). Plus généralement, si $X$ est une $K$-variété analytique rigide, on a les sous-faisceaux $\mathcal{O}_{X}^{\circ}, \mathcal{O}_{X}^{\vee} \subset \mathcal{O}_{X}$ du faisceau structural. Le premier est un faisceau de $K^{\circ}$-algèbres et le second est un idéal du premier. Si $X=\operatorname{Spm}(A)$, alors $\Gamma\left(X, \mathcal{O}^{\circ}\right)=A^{\circ}$ et $\Gamma\left(X, \mathcal{O}^{\vee}\right)=A^{\vee}$. Enfin, on pose $\tilde{A}=A^{\circ} / A^{\vee}$. C'est l'anneau résiduel de la $K$-algèbre affinoïde $A$. On sait que $\tilde{A}$ est une $k$-algèbre réduite et de type fini.

On considère le foncteur

$$
\mathscr{D}: \mathrm{SmAfnd} / K \longrightarrow \mathrm{Sch} / K^{\circ}
$$

qui envoie un $K$-affinoïde lisse $D$ sur le $K^{\circ}$-schéma $\operatorname{Spec}\left(\Gamma\left(D, \mathcal{O}^{\circ}\right)\right.$ ). (Ici, SmAfnd/ $K$ est la catégorie des $K$-affinoïdes lisses et $\mathrm{Sch} / K^{\circ}$ est la catégorie des $K^{\circ}$-schémas qui ne sont pas nécessairement de type fini.) On considère $\mathscr{D}$ comme un diagramme de schémas. On pose $\mathscr{D}_{\eta}=\mathscr{D} \otimes_{K^{\circ}} K$ et $\mathscr{D}_{\sigma}=\left(\mathscr{D} \otimes_{K^{\circ}} k\right)_{\text {red }}$. (Remarquer que $\mathscr{D}_{\sigma}(\operatorname{Spm}(A))=\operatorname{Spec}(\tilde{A})$ de sorte que $\mathscr{D}_{\sigma}$ est un diagramme de $k$-schémas de type fini.) On a ainsi un diagramme commutatif (dans la catégorie des diagrammes de schémas) à carrés cartésiens (à nil-immersions près) :

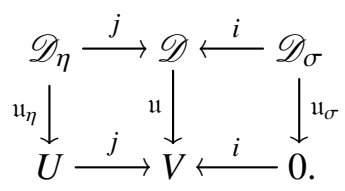

Rappelons que $\mathrm{Sm} / \mathscr{D}$ (resp. $\mathrm{Sm} / \mathscr{D}_{\eta}, \mathrm{Sm} / \mathscr{D}_{\sigma}$ ) est la catégorie dont les objets sont les couples $(X, D)$ où $D$ est un $K$-affinoïde lisse et $X$ un schéma lisse sur $\mathscr{D}(D)\left(\right.$ resp. $\left.\mathscr{D}_{\eta}(D), \mathscr{D}_{\sigma}(D)\right)$. On dispose d'un foncteur « diagonal » diag : SmAfnd $K \longrightarrow \mathrm{Sm} / \mathscr{D}$ qui envoie un $K$-affinoïde $D$ sur le couple $\left(\operatorname{id}_{\mathscr{D}(D)}, D\right)$. On définit de même les foncteurs diagonaux $\operatorname{diag}_{\eta}$ et diag ${ }_{\sigma}$ à valeurs dans $\mathrm{Sm} / \mathscr{D}_{\eta}$ et $\mathrm{Sm} / \mathscr{D}_{\sigma}$. Ils envoient $D$ sur $\left(\operatorname{id}_{\mathscr{D}_{\eta}(D)}, D\right)$ et $\left(\operatorname{id}_{\mathscr{D}_{\sigma}(D)}, D\right)$ respectivement. On note diag*, $\operatorname{diag}_{\eta}^{*}$ et $\operatorname{diag}_{\sigma}^{*}$ les foncteurs images directes suivant ces foncteurs. Les foncteurs « diagonaux » passent aux catégories des $T$-spectres. Pour plus de détails, le lecteur est renvoyé à [7, §1.3.4]. 
Si $L$ est un complexe de préfaisceaux sur $\mathrm{Sm} / U$, on a un isomorphisme canonique

$$
\mathrm{r}_{*} \operatorname{Rig}^{*} L \simeq \operatorname{diag}_{\eta}^{*} \mathfrak{u}_{\eta}^{*} L
$$

où $\mathrm{r}_{*}$ désigne la restriction d'un préfaisceau sur $\mathrm{SmRig} / K$ à $S m A f n d / K$. (On renvoie le lecteur à la preuve du Théorème 2.24 pour la différence entre « Rig* » et « Rig $^{*} »$.) Cet isomorphisme naturel s'étend aux $T$-spectres. On peut former la composition de

$$
\mathrm{r}_{*} \operatorname{Rig}^{*} \mathbf{L} \simeq \operatorname{diag}_{\eta}^{*} u_{\eta}^{*} \mathbf{L} \longrightarrow \operatorname{Rdiag}^{*} \mathrm{R} j_{*} u_{\eta}^{*} \mathbf{L} \longrightarrow \operatorname{Rdiag}^{*} i_{*} L i^{*} \mathrm{R} j_{*} u_{\eta}^{*} \mathbf{L} \simeq \operatorname{Rdiag}_{\sigma}^{*} L i^{*} \mathrm{R} j_{*} u_{\eta}^{*} \mathbf{L}
$$

D'arpès [7, Th. 1.3.37 et 1.3.38], cette composition est une équivalence $\left(\mathbb{B}^{1}\right.$, Nis)-locale et le but est un $T$-spectre projectivement stablement $\left(\mathbb{B}^{1}\right.$, Nis)-fibrant.

Pour la suite de la preuve, il sera commode d'expliciter (173). On fixe un remplacement projectivement stablement $\left(\mathbb{A}^{1}\right.$, Nis)-fibrant $\mathfrak{u}_{\eta}^{*} \mathbf{L} \longrightarrow \widetilde{\mathbf{L}}$. On fixe aussi un morphisme $\widetilde{\mathbf{L}}^{\prime \prime} \longrightarrow j_{*} \widetilde{\mathbf{L}}^{\prime}$ qui est un quasi-isomorphisme niveau par niveau et tel que $\widetilde{\mathbf{L}}^{\prime \prime}$ est projectivement cofibrant. Pour un $K$-affinoïde lisse $D=\operatorname{Spm}(A),\left.\mathfrak{u}_{\eta}(D)^{*} \mathbf{L} \longrightarrow \widetilde{\mathbf{L}}^{\prime}\right|_{\mathrm{Sm} / A}$ est un remplacement projectivement stablement $\left(\mathbb{A}^{1}\right.$, Nis)-fibrant, et $\left.\left.\widetilde{\mathbf{L}}^{\prime \prime}\right|_{\mathrm{Sm} / A^{\circ}} \longrightarrow j_{*} \widetilde{\mathbf{L}}^{\prime}\right|_{\mathrm{Sm} / A}$ est un quasi-isomorphisme niveau par niveau et de source un $T$-spectre projectivement cofibrant. En appliquant $\Gamma\left(D, \operatorname{Ev}_{n}(-)\right)$ à la composition de (173), on obtient la composition de

$$
\begin{aligned}
& \mathbf{L}_{n}(A) \longrightarrow \Gamma\left(A,\left.\left(\widetilde{\mathbf{L}}_{n}^{\prime}\right)\right|_{\mathrm{Sm} / A}\right)=\Gamma\left(A^{\circ},\left.j_{*}\left(\widetilde{\mathbf{L}}_{n}^{\prime}\right)\right|_{\mathrm{Sm} / A}\right) \stackrel{\text { q.i. }}{\longleftarrow} \Gamma\left(A^{\circ},\left.\left(\widetilde{\mathbf{L}}_{n}^{\prime \prime}\right)\right|_{\mathrm{Sm} / A^{\circ}}\right) \\
& \longrightarrow \Gamma\left(\tilde{A},\left.i^{*}\left(\widetilde{\mathbf{L}}_{n}^{\prime \prime}\right)\right|_{\mathrm{Sm} / A^{\circ}}\right) \longrightarrow \Gamma\left(\tilde{A},\left.\operatorname{REv}_{n} i^{*}\left(\widetilde{\mathbf{L}}^{\prime \prime}\right)\right|_{\mathrm{Sm} / A^{\circ}}\right) \text {. }
\end{aligned}
$$

Ci-dessus, nous avons noté $\mathbf{L}_{n}(A)$ la colimite des $\mathbf{L}_{n}(\operatorname{Spec}(B))$ où $B$ parcourt l'ensemble filtrant des sous- $\mathcal{O}(U)$-algèbres lisses de $A$.

Étape $C$ : D'après la troisième étape de la preuve du Théorème 2.24 , le motif $\chi(F / U)^{*} \mathbf{L}$ est donné par la construction suivante : si $\operatorname{Rig}^{*}(\mathbf{L}) \longrightarrow \mathbf{M}$ est un remplacement stablement $\left(\mathbb{A}^{1}\right.$, Nis)-fibrant (par exemple, celui donné par (173)), alors $\chi(F / U)^{*} \mathbf{L}$ est canoniquement isomorphe au $T$-spectre $\left\{\mathbf{M}_{n}\left(Q^{r i g}(-)\right)\right\}_{n \in \mathbb{N}}$. En prenant le remplacement stablement ( $\mathbb{A}^{1}$, Nis)-fibrant donné par (173), on déduit un isomorphisme canonique

$$
\chi(F / U)^{*} \mathbf{L} \simeq\left\{\operatorname{Spec}(\tilde{A}) \in \operatorname{Sm}^{\mathrm{af}} / k \rightsquigarrow \Gamma\left(\tilde{A},\left.\operatorname{REv}_{n} i^{*}\left(\widetilde{\mathbf{L}}^{\prime \prime}\right)\right|_{\operatorname{Sm} / \tilde{A}[[\varpi]]}\right)\right\}_{n \in \mathbb{N}} .
$$

De plus, le morphisme $\mathfrak{Q}_{*}(\mathbf{L}) \longrightarrow \chi(F / U)^{*} \mathbf{L}$, en niveau $n \in \mathbb{N}$ et sur les sections au-dessus de $\operatorname{Spec}(\tilde{A})$, est donné par le morphisme $\mathbf{L}_{n}\left(Q_{\mathrm{ett}}^{r i g}(\tilde{A})\right) \longrightarrow \mathbf{L}_{n}\left(\tilde{A}[[\varpi]]\left[\varpi^{-1}\right]\right)$ suivi par la composition $\operatorname{de}(174)$ avec $A=\tilde{A}[[\varpi]]\left[\varpi^{-1}\right]$.

Par ailleurs, on a morphisme canonique

$$
\Gamma\left(\tilde{A}, \operatorname{REv}_{n} i^{*} \mathbf{L}^{\prime \prime}\right) \longrightarrow \Gamma\left(\tilde{A},\left.\operatorname{REv}_{n} i^{*}\left(\widetilde{\mathbf{L}}^{\prime \prime}\right)\right|_{\mathrm{Sm} / \tilde{A}[[\varpi]]}\right)
$$

naturel en $\operatorname{Spec}(\tilde{A}) \in \operatorname{Sm}^{\text {af }} / k$. C'est un quasi-isomorphisme. En effet, il s'identifie à

$$
\mathrm{R} \Gamma\left(\tilde{A}, \mathrm{Ev}_{n} i^{*} j_{*} \mathbf{L}\right) \longrightarrow \mathrm{R} \Gamma\left(\tilde{A}, \operatorname{Ev}_{n} i^{*} j_{*}\left(\mathfrak{u}_{\eta}\left(Q^{r i g}(\tilde{A})\right)\right)^{*} \mathbf{L}\right)
$$

et ce morphisme est inversible d'après [7, Cor. 1.A.5]. (Pour un $K$-affinoïde lisse $D$, on note $\mathfrak{u}_{\eta}(D)$ le morphisme $\mathscr{D}_{\eta}(D) \longrightarrow U$.) L'isomorphisme (175) et les inverses des quasi-isomorphismes (176) induisent un isomorphisme dans $\mathbf{D A}(k)$ :

$$
\chi(F / U)^{*} \mathbf{L} \simeq\left\{\operatorname{Spec}(\tilde{A}) \in \operatorname{Sm}^{\mathrm{af}} / k \rightsquigarrow \Gamma\left(\tilde{A}, \operatorname{REv}_{n} i^{*} \mathbf{L}^{\prime \prime}\right)\right\}_{n \in \mathbb{N}}=\chi_{U} \mathbf{L} .
$$

Une inspection de la preuve de la première moitié de [7, Scholie 1.3.26, (2)] montre que l'isomorphisme $\chi(F / U)^{*} \mathbf{L} \simeq \chi_{U} \mathbf{L}$ donné par loc. cit. et que nous avions utilisé auparavant (par exemple, dans la preuve de la Proposition 4.28) coïncide avec celui que l'on vient de décrire, i.e., avec (177).

Nous sommes maintenant en mesure de conclure. En effet, d'après ce qui précède, la composition de

$$
\mathfrak{Q}_{*}(\mathbf{L}) \longrightarrow \chi(F / U)^{*} \mathbf{L} \simeq \chi_{U} \mathbf{L}
$$

est donnée, en niveau $n \in \mathbb{N}$ et sur les sections au-dessus $\operatorname{de} \operatorname{Spec}(\tilde{A}) \in \mathrm{Sm}^{\text {af }} / k$, par le morphisme $\mathbf{L}_{n}\left(Q_{\mathrm{e} t}^{\text {rig }}(\tilde{A})\right) \longrightarrow \mathbf{L}_{n}(A)\left(\operatorname{avec} A=\tilde{A}[[\varpi]]\left[\varpi^{-1}\right]\right)$ suivi par la composition de (174) qui est suivie 
par un inverse (dans $\mathbf{D}(\mathbb{Q})$ ) du quasi-isomorphisme (176). Or, il est facile de voir qu'on a un carré commutatif

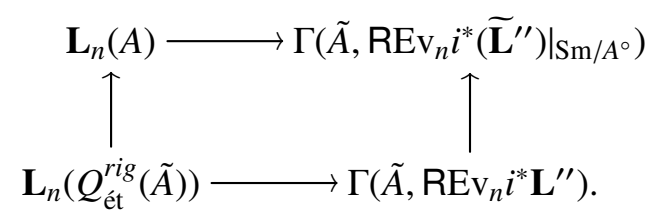

Il s'ensuit aussitôt que la composition de (178) est aussi donnée, en niveau $n \in \mathbb{N}$ et sur les sections au-dessus $\operatorname{de} \operatorname{Spec}(\tilde{A}) \in \mathrm{Sm}^{\text {af }} / k$, par la composition de

$$
\mathbf{L}_{n}\left(Q_{\mathrm{et}}^{r i g}(\tilde{A})\right) \longrightarrow \mathbf{L}_{n}^{\prime}\left(Q_{\mathrm{et}}^{r i g}(\tilde{A})\right) \longrightarrow \Gamma\left(\tilde{A}, \operatorname{REv}_{n} i^{*} \mathbf{L}^{\prime \prime}\right) .
$$

Ceci termine la preuve de la proposition.

Remarque 4.32 - La Proposition 4.31 entraine que la seconde composition dans (171) est un isomorphisme dans $\mathbf{D M}^{e f f}(k)$. Il s'ensuit que la transformation naturelle $\chi_{U}^{\text {bete }} \longrightarrow \chi_{U}$ admet une section canonique, i.e., $\chi_{U}$ est canoniquement un facteur direct de $\chi_{U}^{\text {bête }}$. Peut-être est-il loisible de conjecturer que $\chi_{U}^{\text {bête }} \longrightarrow \chi_{U}$ est un isomorphisme.

Reprenons à présent les notations du §4.4. Posons $N=\mathbb{Q}_{t r}(Y / \partial Y)$ et $N_{0}=\mathbb{Q}_{t r}\left(Y_{0} / \partial Y_{0}\right)$. Ce sont des objets dans $\mathbf{D M}^{e f f}(V)$ et $\mathbf{D M}^{e f f}(k)$. De plus, on a les identifications $i^{*} N \simeq N_{0}$ et $j^{*} N \simeq M$. On dispose d'un morphisme canonique

$$
N_{0} \longrightarrow \chi_{U}(M)
$$

donné par la composition de

$$
N_{0} \simeq i^{*} N \longrightarrow i^{*} j_{*} j^{*} N \simeq \chi_{U}^{\text {bête }} M \longrightarrow \chi_{U} M .
$$

Par ailleurs, le morphisme $b_{0}$ de (159) définit une classe d'homologie $\beta_{0} \in \mathrm{H}_{n} \mathrm{C}\left(N_{0}\left(\overline{\mathbb{D}}_{\text {ét }}\right)\right) \simeq$ $\mathrm{H}_{n} \mathrm{Bti}^{*} N_{0}$. On a le résultat suivant.

Corollaire 4.33 - L'image de $\gamma$ par l'isomorphisme $\mathrm{Bti}^{*} \chi(F / U)^{*} M \simeq \mathrm{Bti}^{*} \chi_{U} M$ correspond à la composition de

$$
\mathbb{Q}[n] \stackrel{\beta_{0}}{\longrightarrow} \mathrm{Bti}^{*} N_{0} \longrightarrow \mathrm{Bti}^{*} \chi_{U} M .
$$

Demonstration Rappelons que $\gamma$ est la composition de

$$
\mathbb{Q}[n] \stackrel{\alpha}{\longrightarrow} \mathrm{Bti}^{*} \mathfrak{Q}_{*}(M) \simeq \mathrm{Bti}^{*} \chi(F / U)^{*} M
$$

où $\alpha \in \mathrm{H}_{n} \mathrm{C}\left(\mathfrak{Q}_{*}(M)\left(\overline{\mathbb{D}}_{\text {ét }}\right)\right) \simeq \mathrm{H}_{n} \mathrm{Bti}^{*} \mathfrak{Q}_{*}(M)$ est la classe induite par $a: \overline{\mathbb{D}}_{\text {êt }}^{\dagger, n} \longrightarrow X$. Ainsi, l'image de $\gamma$ par l'isomorphisme $\mathrm{Bti}^{*} \chi(F / U)^{*} M \simeq \mathrm{Bti}^{*} \chi_{U} M$ correspond à la composition de

$$
\mathbb{Q}[n] \stackrel{\alpha}{\longrightarrow} \operatorname{Bti}^{*} \mathfrak{Q}_{*}(M) \simeq \mathrm{Bti}^{*} \chi(F / U)^{*} M \simeq \mathrm{Bti}^{*} \chi_{U} M .
$$

D'après la Proposition 4.31, c'est aussi la composition de

$$
\mathbb{Q}[n] \stackrel{\alpha}{\longrightarrow} \operatorname{Bti}^{*} \mathfrak{Q}_{*}(M) \longrightarrow \mathrm{Bti}^{*} \mathfrak{Q}_{*}\left(M^{\prime}\right) \longrightarrow \mathrm{Bti}^{*} \chi_{U}^{\text {bete }} M \longrightarrow \mathrm{Bti}^{*} \chi_{U} M .
$$

Ci-dessus, nous avons choisi un remplacement projectivement ( $\mathbb{A}^{1}$,ét)-fibrant $M \longrightarrow M^{\prime}$ et un quasi-isomorphisme $M^{\prime \prime} \longrightarrow j_{*} M^{\prime}$ avec $M^{\prime \prime}$ projectivement cofibrant; la troisième flèche est alors donnée par le zigzag

$$
\mathfrak{Q}_{*}\left(M^{\prime}\right)=j_{*} M^{\prime}\left(Q_{\mathrm{e} t}^{f o r}(-)\right) \stackrel{\text { q.i. }}{\longleftarrow} M^{\prime \prime}\left(Q_{\mathrm{ett}}^{f o r}(-)\right) \longrightarrow i^{*} M^{\prime \prime}(-) .
$$

On dispose d'un morphisme canonique $Q_{\mathrm{èt}}^{f o r}\left(Y_{0}\right) \longrightarrow Y$ qui induit un morphisme $Q_{\mathrm{e} t}^{r i g}\left(Y_{0}\right) \longrightarrow X$. Il s'ensuit un morphisme canonique :

$$
N_{0}=\mathbb{Q}_{t r}\left(Y_{0} / \partial Y_{0}\right) \longrightarrow \mathbb{Q}_{*}\left(\mathbb{Q}_{t r}(X / \partial X)\right)=\mathfrak{Q}_{*}(M) .
$$


Étant donné que le carré suivant commute

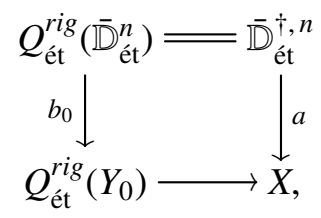

on déduit aussitôt un carré commutatif

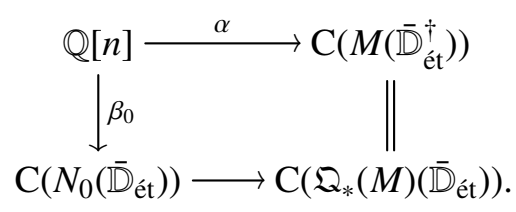

La composition de (180) est alors égale à celle de

$$
\mathbb{Q}[n] \stackrel{\beta_{0}}{\longrightarrow} \mathrm{Bti}^{*} N_{0} \longrightarrow \mathrm{Bti}^{*} \mathfrak{Q}_{*}(M) \longrightarrow \mathrm{Bti}^{*} \mathfrak{Q}_{*}\left(M^{\prime}\right) \longrightarrow \mathrm{Bti}^{*} \chi_{U}^{\text {bête }} M \longrightarrow \mathrm{Bti}^{*} \chi_{U} M .
$$

Il reste à identifier la composition de

$$
N_{0} \longrightarrow \mathfrak{Q}_{*}(M) \longrightarrow \mathfrak{Q}_{*}\left(M^{\prime}\right) \longrightarrow \chi_{U}^{\text {bête }} M \longrightarrow \chi_{U} M
$$

avec celle de (179). Pour cela, nous allons supposer que le quasi-isomorphisme $M^{\prime \prime} \longrightarrow j_{*} M^{\prime}$ est une fibration injective. Le morphisme $N_{0} \longrightarrow \mathfrak{Q}_{*}\left(M^{\prime}\right)$ correspond à la composition de

$$
\mathbb{Q}_{t r}\left(Q_{\mathrm{et}}^{f o r}\left(Y_{0}\right) / Q_{\mathrm{et}}^{f o r}\left(\partial Y_{0}\right)\right) \longrightarrow \mathbb{Q}_{t r}(Y / \partial Y) \longrightarrow j_{*} M^{\prime} .
$$

(La source du premier morphisme ci-dessus est un pro-objet dans la catégorie des préfaisceaux avec transferts et le second morphisme est le morphisme tautologique suivi de $j_{*} M \longrightarrow j_{*} M^{\prime}$.) Puisque $M^{\prime \prime} \longrightarrow j_{*} M^{\prime}$ est une fibration triviale injective, il existe un morphisme $\mathbb{Q}_{t r}(Y / \partial Y) \longrightarrow M^{\prime \prime}$ faisant commuter le triangle

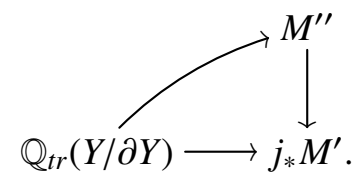

On en déduit un morphisme $N_{0} \longrightarrow M^{\prime \prime}\left(Q_{\text {êt }}^{f o r}(-)\right)$ qui correspond à la composition de

$$
\mathbb{Q}_{t r}\left(Q_{\mathrm{et}}^{f o r}\left(Y_{0}\right) / Q_{\mathrm{e} t}^{f o r}\left(\partial Y_{0}\right)\right) \longrightarrow \mathbb{Q}_{t r}(Y / \partial Y) \longrightarrow M^{\prime \prime} .
$$

En composant avec $M^{\prime \prime}\left(Q_{\mathrm{et}}^{f o r}(-)\right) \longrightarrow i^{*} M^{\prime \prime}$, on obtient un morphisme $N_{0} \longrightarrow i^{*} M^{\prime \prime}$ égal à la composition de

$$
N_{0} \simeq i^{*} \mathbb{Q}_{t r}\left(Q_{\mathrm{et}}^{f o r}\left(Y_{0}\right) / Q_{\mathrm{et}}^{f o r}\left(\partial Y_{0}\right)\right) \longrightarrow i^{*} \mathbb{Q}_{t r}(Y / \partial Y) \longrightarrow i^{*} M^{\prime \prime}
$$

qui est clairement égale à la composition de $N_{0} \simeq i^{*} N \longrightarrow i^{*} M^{\prime \prime}=\chi_{U}^{\text {bête }} M$. Ceci termine la preuve du corollaire.

Nous allons reprendre la construction qui précède le Corollaire 4.33 dans le contexte analytique complexe. Notons $M^{a n}=\mathbb{Q}_{t r}\left(X^{a n} / \partial X^{a n}\right), N^{a n}=\mathbb{Q}_{t r}\left(Y^{a n} / \partial Y^{a n}\right)$ et $N_{0}^{a n}=\mathbb{Q}_{t r}\left(Y_{0}^{a n} / \partial Y_{0}^{a n}\right)$. Ce sont les objets des catégories $\mathbf{A n D M}^{e f f}(-)$ qu'on obtient à partir de $M, N$ et $N_{0}$ par application des foncteurs $\mathrm{An}^{*}: \mathbf{D M}^{e f f}(-) \longrightarrow \mathbf{A n D M}^{e f f}(-)$. On dispose d'un morphisme canonique

$$
N_{0}^{a n} \longrightarrow \chi_{U^{a n}}\left(M^{a n}\right)
$$

donné par la composition de

$$
N_{0}^{a n} \simeq i^{*} N^{a n} \longrightarrow i^{*} j_{*} j^{*} N^{a n} \simeq \chi_{U^{a n}} M^{a n} .
$$

Par ailleurs, le morphisme $\overline{\mathbb{D}}^{n} \longrightarrow Y_{0}^{a n}$ (voir le début du §4.4) définit une classe d'homologie $\beta_{0}^{a n} \in$ $\mathrm{H}_{n} \mathrm{C}\left(N_{0}^{a n}(\bar{D})\right) \simeq \mathrm{H}_{n} R \iota_{*} N_{0}^{a n}$. (On rappelle que pour un espace analytique complexe $S$, on note 
$\iota^{*}: \mathbf{D}(S) \longrightarrow \operatorname{AnDM}^{e f f}(S)$ le foncteur « image inverse » suivant l'inclusion $\operatorname{Ouv}(S) \hookrightarrow \operatorname{AnSm} / S$ et $\mathrm{R} \iota_{*}$ son adjoint à droite ; ce sont des équivalences de catégories inverses l'une de l'autre.)

On a donc le résultat suivant.

Corollaire 4.34 - La composition de (169) est égale à celle de

$$
\mathbb{Q}[n] \stackrel{\beta_{0}^{a n}}{\longrightarrow} \mathrm{R} \iota_{*} N_{0}^{a n} \longrightarrow \mathrm{R} \iota_{*} \chi_{U^{a n}} M^{a n} \simeq \chi_{U^{a n}} \mathrm{R}_{\iota_{*}} M^{a n} .
$$

(Rappelons que $\mathrm{Bti}_{U}^{*} M=\mathrm{R}_{*} M^{\text {an }}$.)

Demonstration Ceci est une conséquence immédiate du Corollaire 4.33 et de la commutation de certaines opérations de Grothendieck avec les foncteurs $\mathrm{An}^{*}$ et $\mathrm{R} \iota_{*}$ (voir [3]).

4.6. Le morphisme d'évaluation, troisième partie. - Dans ce paragraphe on termine la preuve du Théorème 4.21. Dans le $\$ 4.5$, nous avons traité une partie de (168). Nous allons maintenant traiter la partie restante. On considère d'abord la composition de

$$
\begin{aligned}
\chi_{U^{a n} \mathrm{Bti}_{U}^{*}} \operatorname{lis}_{U} \Omega_{/ U}^{\cdot} \longrightarrow \chi_{U^{a n}} \mathrm{Bti}_{U}^{*} \operatorname{lis}_{U} \mathrm{Bti}_{U *} \mathrm{G}_{r h}\left(\mathcal{O}_{U_{\mathrm{C}}}\right) \\
\qquad \chi_{U^{a n}} \mathrm{G}_{r h}\left(\mathcal{O}_{U_{\mathrm{C}}}\right) \longrightarrow \Psi_{U^{a n}} \mathrm{G}_{r h}\left(\mathcal{O}_{U_{\mathrm{C}}}\right) \simeq \mathcal{A}_{U_{\mathrm{C}}}^{\mathrm{rs}}\left(\mathcal{O}_{U_{\mathrm{C}}}\right) \hookrightarrow \mathcal{A} .
\end{aligned}
$$

D'après la Proposition 4.8, on dispose d'un morphisme $\mathrm{G}_{r h}\left(\mathcal{O}_{U_{\mathbb{C}}}\right) \longrightarrow \mathcal{O}_{U^{a n}}$ et la composition des deux derniers morphismes dans (184) est égale à celle de $\Psi_{U^{a n}} \mathrm{G}_{r h}\left(\mathcal{O}_{U_{\mathbb{C}}}\right) \longrightarrow \Psi_{U^{a n}} \mathcal{O}_{U^{a n}} \simeq \mathcal{A}$. Il s'ensuit que la composition de (184) est égale à celle de

$$
\begin{aligned}
\chi_{U^{a n} \mathrm{Bti}_{U}^{*} \operatorname{lis}_{U} \Omega_{/ U}^{\cdot}} \longrightarrow \chi_{U^{a n} \mathrm{Bti}_{U}^{*} \operatorname{lis}_{U} \mathrm{Bti}_{U *} \mathrm{G}_{r h}\left(\mathcal{O}_{U_{\mathbb{C}}}\right)} \\
\longrightarrow \chi_{U^{a n}} \mathrm{G}_{r h}\left(\mathcal{O}_{U_{\mathbb{C}}}\right) \longrightarrow \chi_{U^{a n}} \mathcal{O}_{U^{a n}} \hookrightarrow \mathcal{A},
\end{aligned}
$$

mais aussi à celle de

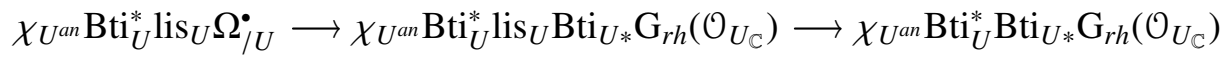

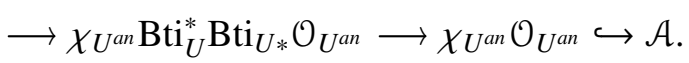

En utilisant la Proposition 4.15, on déduit que la composition de (186) est égale à celle de

$$
\begin{aligned}
& \chi_{U^{a n} \mathrm{Bti}_{U}^{*}} \operatorname{lis}_{U} \Omega_{/ U}^{*} \longrightarrow \chi_{U^{a n}} \mathrm{Bti}_{U}^{*} \Omega_{/ U}^{*} \longrightarrow \chi_{U^{a n}} \mathrm{Bti}_{U}^{*} \mathrm{An}_{*} \Omega_{/ U^{a n}}^{*} \\
& \simeq \chi_{U^{a n}} \mathrm{Bti}_{U}^{*} \mathrm{An}_{*} \iota^{*} \mathcal{O}_{U^{a n}} \longrightarrow \chi_{U^{a n}} \mathcal{O}_{U^{a n}} \hookrightarrow \mathcal{A},
\end{aligned}
$$

mais aussi à celle de

$$
\begin{aligned}
\chi_{U^{a n}} \mathrm{R}_{\iota_{*}} \operatorname{An}^{*} \operatorname{lis}_{U} \Omega_{/ U}^{*} \longrightarrow \chi_{U^{a n}} \mathrm{R}_{*} \mathrm{An}^{*} \Omega_{/ U}^{\circ} \longrightarrow & \chi_{U^{a n}} \mathrm{R}_{\iota_{*}} \operatorname{An}^{*} \mathrm{An}_{*} \Omega_{/ U^{a n}}^{*} \\
& \longrightarrow \chi_{U^{a n}} \mathrm{R}_{\iota_{*}} \Omega_{U^{a n}}^{\cdot} \simeq \chi_{U^{a n}} \mathcal{O}_{U^{a n}} \hookrightarrow \mathcal{A} .
\end{aligned}
$$

Rappelons que nous nous sommes donnés une forme différentielle relative $\omega \in \Omega_{/ U}^{n}(X)$ induisant le morphisme $\omega: M[-n] \longrightarrow \Omega_{j U}^{\cdot}$ dans $\mathbf{D M}^{e f f}(U)$ (voir le début du §4.4). De même, $\omega^{a n} \in \Omega_{/ U^{a n}}^{n}\left(X^{a n}\right)$, la forme différentielle relative analytique déduite de $\omega$, définit un morphisme $\omega^{a n}: M^{a n}[-n] \longrightarrow \Omega_{U^{a n}}^{\cdot a n s} \mathbf{A n D M}^{e f f}\left(U^{a n}\right)$. Il est alors clair que le diagramme suivant est commutatif

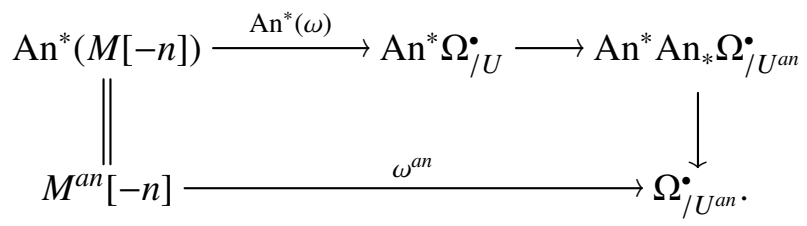

De ce qui précède, on déduit immédiatement que la composition des flèches dans (168) qui se trouvent entre $\chi_{U^{a n} \mathrm{Bti}_{U}^{*}}^{*} M[-n]$ et $\mathcal{A}_{U_{\mathbb{C}}}^{\mathrm{rs}}\left(U_{\mathbb{C}}\right)$, suivie de l'inclusion $\mathcal{A}_{U_{\mathbb{C}}}^{\mathrm{rs}}\left(U_{\mathbb{C}}\right) \hookrightarrow \mathcal{A}$, est égale à celle de

$$
\chi_{U^{a n}} \mathrm{R} \iota_{*} M^{a n}[-n] \stackrel{\omega^{a n}}{\longrightarrow} \chi_{U^{a n}} \mathrm{R} \iota_{*} \Omega_{J^{a n}}^{\cdot} \simeq \chi_{U^{a n}} \mathcal{O}_{U^{a n}} \hookrightarrow \mathcal{A} .
$$

En joignant ceci au Corollaire 4.34, on obtient le résultat suivant. 
Proposition 4.35 - La composition de (168), suivie de l'inclusion $\mathcal{A}_{U_{\mathbb{C}}}^{\mathrm{rs}}\left(U_{\mathbb{C}}\right) \hookrightarrow \mathcal{A}$, est égale à celle de

$$
\mathbb{Q} \stackrel{\beta_{0}^{a n}}{\longrightarrow} \mathrm{R} \iota_{*} N_{0}^{a n}[-n] \longrightarrow \chi_{U^{a n}} \mathrm{R} \iota_{*} M^{a n}[-n] \stackrel{\omega^{a n}}{\longrightarrow} \chi_{U^{a n}} \mathrm{R}_{\iota_{*}} \Omega_{J^{a n}}^{\cdot} \simeq \chi_{U^{a n}} \mathcal{O}_{U^{a n}} \hookrightarrow \mathcal{A} .
$$

Comme d'habitude, on note $D_{r} \subset \mathbb{C}$ le disque de centre 0 et rayon $r>0$. Pour $\epsilon>0$ un réel suffisamment petit, la composante connexe de $v^{-1}\left(D_{1+\epsilon}^{n} \times D_{\epsilon}\right) \subset Y^{a n}$ contenant l'image de $\overline{\mathbb{D}}^{n} \longrightarrow Y_{0}^{a n}$ est isomorphe à $D_{1+\epsilon}^{n} \times D_{\epsilon}$; l'isomorphisme étant induit par $v: Y^{a n} \longrightarrow \mathbb{C}^{n} \times V^{a n}$. Ainsi, il existe un morphisme d'espaces analytiques complexes

$$
D_{1+\epsilon}^{n} \times D_{\epsilon} \hookrightarrow Y^{a n}
$$

rendant le diagramme suivant commutatif

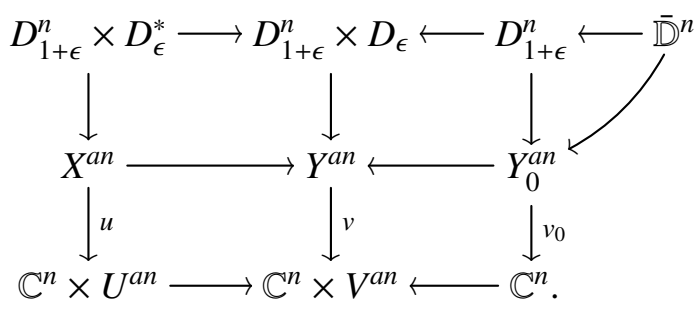

On définit $\partial D_{1+\epsilon}^{n} \subset D_{1+\epsilon}^{n}$ par l'équation $\prod_{i=1}^{n} z_{i}\left(z_{i}-1\right)=0$. On pose alors :

$\bar{M}=\mathbb{Q}_{t r}\left(D_{1+\epsilon}^{n} \times D_{\epsilon}^{*} / \partial D_{1+\epsilon}^{n} \times D_{\epsilon}^{*}\right), \quad \bar{N}=\mathbb{Q}_{t r}\left(D_{1+\epsilon}^{n} \times D_{\epsilon} / \partial D_{1+\epsilon}^{n} \times D_{\epsilon}\right)$ et $\bar{N}_{0}=\mathbb{Q}_{t r}\left(D_{1+\epsilon}^{n} / \partial D_{1+\epsilon}^{n}\right)$.

Ce sont des objets de $\mathbf{A n D M}^{\text {eff }}\left(D_{\epsilon}^{*}\right), \mathbf{A n D M}^{\text {eff }}\left(D_{\epsilon}\right)$ et $\mathbf{A n D M}^{\text {eff }}(p t)$ respectivement. De plus, on a des morphismes évidents $\left.\bar{M} \longrightarrow M^{a n}\right|_{D_{\epsilon}^{*}},\left.\bar{N} \longrightarrow N^{a n}\right|_{D_{\epsilon}}$ et $\bar{N}_{0} \longrightarrow N_{0}^{a n}$.

La classe $\bar{\beta}_{0} \in \mathrm{H}_{n} \mathrm{C}\left(\bar{N}_{0}(\overline{\mathbb{D}})\right) \simeq \mathrm{H}_{n} \mathrm{R} \iota_{*} \bar{N}_{0}$ induite par l'inclusion $\overline{\mathbb{D}}^{n} \hookrightarrow D_{1+\epsilon}^{n}$ rend le triangle suivant

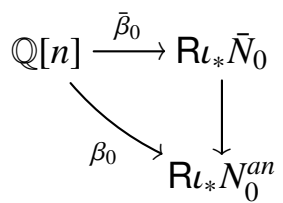

commutatif. On note $\bar{\omega} \in \Omega_{/ D_{\epsilon}^{*}}^{n}\left(D_{1+\epsilon}^{n} \times D_{\epsilon}^{*}\right)$ la forme différentielle relative obtenue par restriction de $\omega^{a n} \in \Omega_{/ U^{a n}}^{n}\left(X^{a n}\right)$; elle définit un morphisme $\bar{\omega}: \bar{M} \longrightarrow \Omega_{/ D_{\epsilon}^{*}}$ dans $\operatorname{AnDM}^{e f f}\left(D_{\epsilon}^{*}\right)$. Il est clair que la composition de (190) est aussi égale à celle de

$$
\mathbb{Q} \stackrel{\bar{\beta}_{0}}{\longrightarrow} \mathrm{R} \iota_{*} \bar{N}_{0}[-n] \longrightarrow \chi_{D_{\epsilon}^{*}} \mathrm{R} \iota_{*} \bar{M}[-n] \stackrel{\bar{\omega}}{\longrightarrow} \chi_{D_{\epsilon}^{*}} \mathrm{R}_{\iota_{*}} \Omega_{/ D_{\epsilon}^{*}}^{\cdot} \simeq \chi_{D_{\epsilon}^{*}} \mathcal{O}_{D_{\epsilon}^{*}} \hookrightarrow \mathcal{A} .
$$

Remarquons à présent que $\bar{\omega}=\bar{G} \cdot \mathrm{d} z_{1} \wedge \cdots \wedge \mathrm{d} z_{n}$ avec $\bar{G} \in \Gamma\left(D_{1+\epsilon}^{n} \times D_{\epsilon}^{*}, \mathcal{O}\right)$ la restriction de la fonction analytique $G^{a n} \in \Gamma\left(X^{a n}, \mathcal{O}\right)$ déduite de la fonction régulière $G$. La fonction $\bar{G}$ admet un développement en série de Laurent par rapport à la variable $\varpi$ de $D_{\epsilon}^{*}$. Ce développement est le même que celui de $G \circ a \in \mathcal{O}_{k-a l g}^{\dagger}\left(\overline{\mathbb{D}}^{n}\right)$. Vu le Lemme 4.30, il reste à démontrer le résultat suivant pour terminer la preuve du Théorème 4.21.

LeMme 4.36 - La composition de (191) envoie $1 \in \mathbb{Q}$ sur $\int_{[0,1]^{n}} \bar{G}$.

Demonstration On divise la preuve en deux parties. Dans la première, nous présentons quelques constructions qui, pour l'essentiel, sont bien connues. Dans la seconde, on applique ces constructions pour obtenir le résultat recherché.

Partie A : Notons $Q=\mathbb{Q}_{t r}\left(D_{1+\epsilon} / \partial D_{1+\epsilon}\right)$; c'est un complexe de préfaisceaux avec transferts sur AnSm/pt. Considérons le morphisme $Q \longrightarrow \mathbb{Q}_{c s t}[1] \oplus \mathbb{Q}_{c s t}[1]$ obtenu en formant le triangle distingué

$$
\mathbb{Q}_{c s t} \oplus \mathbb{Q}_{c s t} \stackrel{0+1}{\longrightarrow} \mathbb{Q}_{t r}\left(D_{1+\epsilon}\right) \longrightarrow Q \longrightarrow \mathbb{Q}_{c s t}[1] \oplus \mathbb{Q}_{c s t}[1] .
$$


(Ci-dessus, $\mathbb{Q}_{c s t}$ désigne le préfaisceau constant de valeur $\mathbb{Q}$ sur $\mathrm{AnSm} / p t$.) On définit alors un morphisme (de complexes à homotopie près) $Q \longrightarrow \mathbb{Q}_{c s t}[1]$ en prenant la composition de

$$
Q \longrightarrow \mathbb{Q}_{c s t}[1] \oplus \mathbb{Q}_{c s t}[1] \stackrel{a \oplus b \mapsto-b}{\longrightarrow} \mathbb{Q}_{c s t}[1] .
$$

Le morphisme $\overline{\mathbb{D}}^{1} \longrightarrow D_{1+\epsilon}$ définit une classe d'homologie $\delta \in \mathrm{H}_{1} \mathrm{C}(Q(\overline{\mathbb{D}})) \simeq \mathrm{H}_{1} \mathrm{R} \iota_{*} Q$ et le choix du signe dans (193) assure que l'image de $\delta$ par le morphisme $\mathrm{C}(Q(\overline{\mathbb{D}})) \longrightarrow \mathrm{C}\left(\mathbb{Q}_{c s t}(\overline{\mathbb{D}})\right)[1]=\mathbb{Q}[1]$ est égale à $1 \in \mathbb{Q}$. (Pour que ceci soit vrai, on doit adopter les conventions de signes de $[4, \S \mathrm{A} .1]$ relativement aux objets cubiques.) Bien entendu, dans $\mathbf{A n D M}^{\text {eff }}(p t)$, la composition de (193) est un isomorphisme. De plus, son inverse est le morphisme $\mathbb{Q}_{c s t}[1]=\iota^{*} \mathbb{Q}[1] \longrightarrow Q$ déduit par adjonction de $\delta: \mathbb{Q}[1] \longrightarrow \mathbf{R}_{*} Q$.

Notons $q$ la projection de $D_{\epsilon}^{*}$ sur le point. En utilisant ce qui précède, on peut former un isomorphisme dans $\operatorname{AnDM}^{e f f}\left(D_{\epsilon}^{*}\right)$ :

donné par la composition de

$$
\Omega_{/ D_{\epsilon}^{*}}^{*} \longrightarrow \underline{\mathcal{H} \operatorname{Hom}}\left(q^{*} Q, \Omega_{/ D_{\epsilon}^{*}}^{*}\right)[1]
$$

$$
\Omega_{/ D_{\epsilon}^{*}}^{*} \simeq \underline{\mathcal{H} o m}\left(q^{*} \mathbb{Q}_{c s t}, \Omega_{/ D_{\epsilon}^{*}}^{*}\right) \simeq \underline{\mathcal{H} o m}\left(q^{*} Q[-1], \Omega_{/ D_{\epsilon}^{*}}^{*}\right) \simeq \underline{\mathcal{H} o m}\left(q^{*} Q, \Omega_{/ D_{\epsilon}^{*}}^{*}[1] .\right.
$$

On souhaite calculer cet isomorphisme. Pour $W \in \mathrm{AnSm} / D_{\epsilon}^{*}$, on a

$$
\Gamma\left(W, \underline{\mathcal{H} \operatorname{Com}}\left(q^{*} Q, \Omega_{/ D_{\epsilon}^{*}}^{*}\right)\right)=\widetilde{\Omega}_{/ D_{\epsilon}^{*}}^{\cdot}\left(D_{1+\epsilon} \times W\right)
$$

où $\widetilde{\Omega}_{/ D_{\epsilon}^{*}}^{d}\left(D_{1+\epsilon} \times W\right)$ est le sous-espace des formes différentielles relatives de $\Omega_{/ D_{\epsilon}^{*}}^{d}\left(D_{1+\epsilon} \times W\right)$ qui s'annulent après substitution par $z=0$ ou $z=1$ (avec $z$ la coordonnée sur $D_{1+\epsilon}$ ). On a alors une suite exacte

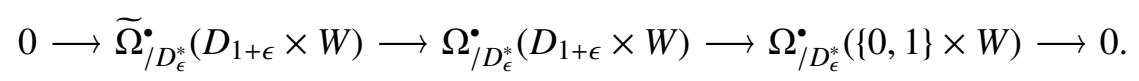

Il s'ensuit un quasi-isomorphisme

$$
\text { Cône }\left\{\widetilde{\Omega}_{/ D_{\epsilon}^{*}}^{\cdot}\left(D_{1+\epsilon} \times W\right) \rightarrow \Omega_{/ D_{\epsilon}^{*}}^{\cdot}\left(D_{1+\epsilon} \times W\right)\right\} \longrightarrow \Omega_{/ D_{\epsilon}^{*}}^{\cdot}(\{0,1\} \times W) .
$$

Il possède un quasi-inverse qui envoie un couple de formes différentielles relatives $\left(u_{0}, u_{1}\right) \in$ $\Omega_{/ D_{\epsilon}^{*}}^{d}(\{0,1\} \times W)$ sur

$$
\left((1-z) \cdot u_{0}+z \cdot u_{1}, \mathrm{~d} z \wedge\left(u_{0}-u_{1}\right)\right) \in \Omega_{/ D_{\epsilon}^{*}}^{d}\left(D_{1+\epsilon} \times W\right) \oplus \widetilde{\Omega}_{/ D_{\epsilon}^{*}}^{d+1}\left(D_{1+\epsilon} \times W\right) .
$$

Il s'ensuit que le morphisme canonique $\Omega_{/ D_{\epsilon}^{*}}^{\cdot}(\{0,1\} \times W) \longrightarrow \widetilde{\Omega}_{/ D_{\epsilon}^{*}}^{\cdot(}\left(D_{1+\epsilon} \times W\right)[1]$, déduit du morphisme connectant dans le triangle (192), est homotope au morphisme de complexes qui envoie $\left(u_{0}, u_{1}\right)$ sur $\mathrm{d} z \wedge\left(u_{0}-u_{1}\right)$. Il découle aussitôt que le morphisme (194) est donné par $u \leadsto \mathrm{d} z \wedge u$ pour $u \in \Omega_{/ D_{\epsilon}^{*}}^{d}(W)$.

Par ailleurs, on dispose d'un morphisme de complexes

$$
\int_{[0,1]}: \underline{\mathcal{H} \operatorname{com}}\left(q^{*} Q, \Omega_{/ D_{\epsilon}^{*}}^{*}[1] \longrightarrow \Omega_{/ D_{\epsilon}^{*}}^{*}\right.
$$

qui envoie une forme différentielle relative $u \in \widetilde{\Omega}_{/ D_{\epsilon}^{*}}^{d+1}\left(D_{1+\epsilon} \times W\right)$ sur son intégrale sur $[0,1] \subset D_{1+\epsilon}^{*}$ (par rapport à la variable $z$ ). Compte tenu du calcul précédent, il est immédiat que (195) est un quasi-inverse à (194).

Partie $B$ : Remarquons qu'on a les identifications $\bar{N}_{0} \simeq Q \otimes \cdots \otimes Q$ ( $n$ fois) et $\bar{M} \simeq q^{*} \bar{N}_{0}$, ainsi que l'égalité $\bar{\beta}_{0}=\delta^{\otimes n}$. En utilisant les constructions de la Partie A, on obtient par induction les faits suivants.

(i) On a un quasi-isomorphisme de complexes de préfaisceaux

$$
\int_{[0,1]^{n}}: \underline{\operatorname{Hom}}\left(\bar{M}, \Omega_{/ D_{\epsilon}^{*}}^{*}\right)[n] \longrightarrow \Omega_{/ D_{\epsilon}^{*}}^{\cdot}
$$

donné par l'intégration. 
(ii) $\operatorname{Dans} \operatorname{AnDM}^{e f f}\left(D_{\epsilon}^{*}\right)$, ce morphisme est égal à la composition de

$$
\underline{\mathcal{H} \text { om }}\left(\bar{M}, \Omega_{/ D_{\epsilon}^{*}}\right)[n] \longrightarrow \underline{\mathcal{H} \text { om }}\left(\mathbb{Q}_{c s t}[n], \Omega_{/ D_{\epsilon}^{*}}\right)[n] \simeq \Omega_{/ D_{\epsilon}^{*}},
$$

où le premier morphisme est induit par le morphisme $\mathbb{Q}_{c s t}[n] \longrightarrow \bar{M}$ qui est induit par l'inclusion $\overline{\mathbb{D}}^{n} \times D_{\epsilon}^{*} \hookrightarrow D_{1+\epsilon}^{n} \times D_{\epsilon}^{*}$.

Il découle aussitôt que la composition de

$$
\iota^{*} \mathbb{Q}=\mathbb{Q}_{c s t} \stackrel{q^{*} \bar{\beta}_{0}^{\prime}}{\longrightarrow} \bar{M}[-n] \stackrel{\bar{\omega}}{\longrightarrow} \Omega_{/ D_{\epsilon}^{*}}^{*} \simeq \iota^{*} \mathcal{O}_{U^{a n}}
$$

envoie $1 \in \mathbb{Q}$ sur $\int_{[0,1]^{n}} \bar{G}$. (Ci-dessus, nous avons noté $\bar{\beta}_{0}^{\prime}: \iota^{*} \mathbb{Q} \longrightarrow \bar{N}_{0}$ le morphisme déduit par adjonction de $\bar{\beta}_{0}$.) En appliquant $\chi_{D_{\epsilon}^{*}}$ et $\mathrm{R} \iota_{*}$ à la composition ci-dessus et en utilisant la commutation du carré

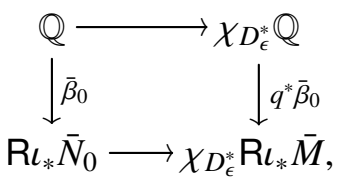

on obtient le résultat recherché.

\section{Références}

[1] J. Ayouв. - Les six opérations de Grothendieck et le formalisme des cycles évanescents dans le monde motivique, I. Astérisque, volume 314, Société Mathématique de France, 2007.

[2] J. Ayoub. - Les six opérations de Grothendieck et le formalisme des cycles évanescents dans le monde motivique, II. Astérisque, volume 315, Société Mathématique de France, 2007.

[3] J. Ayoub. - Note sur les opérations de Grothendieck et la réalisation de Betti. Journal de l'Institut de Mathématiques de Jussieu, (2010) 9(2), p. 225-263.

[4] J. Ayouв. - L'algèbre de Hopf et le groupe de Galois motiviques d'un corps de caractéristique nulle, I. Journal für die reine und angewandte Mathematik (Crelles Journal) 693, 2014, p. 1-149.

[5] J. Ayouв. - L'algèbre de Hopf et le groupe de Galois motiviques d'un corps de caractéristique nulle, II. Journal für die reine und angewandte Mathematik (Crelles Journal) 693, 2014, p. 151-226.

[6] J. Ayoub. - Erratum à L'algèbre de Hopf et le groupe de Galois motiviques d'un corps de caractéristique nulle, II. Journal für die reine und angewandte Mathematik (Crelles Journal) 693, 2014, e1-e2.

[7] J. Ayoub. - Les motifs des variétés analytiques rigides. Mémoires de la Société Mathématique de France, 140, 141 (2015).

[8] A. Borel. - Algebraic D-modules. Perspectives in Mathematics, Vol. 2. Academic Press, Inc., 1987.

[9] D.-C. Cisinski and F. DÉglise. - Local and stable homological algebra in Grothendieck abelian categories. Homology, Homotopy Appl., 11(1), p. 219-260, 2009.

[10] P. Deligne. - Equations différentielles à points singuliers réguliers. Lecture Notes in Math. 163, Springer (1970).

[11] P. Deligne. - Catégories tannakiennes. In The Grothendieck Festschrift, Vol. II. Progress in Math., vol. 87, 1990.

[12] E. M. Friedlander, A. Suslin and V. Voevodsky. - Cycles, transfers, and motivic homology theories. Annals of Mathematics Studies, 143, 2000.

[13] A. Grothendieck. - On the de Rham cohomology of algebraic varieties. Publication Mathématiques de l'I.H.E.S, tome 29 (1966), p. 95-103.

[14] A. Grothendieck et J. Dieudonné. - Éléments de géométrie algébrique III. Étude cohomologique des faisceaux cohérents, Première partie. Publications Mathématiques de l'I.H.E.S, volume 11 (1961), p. 5-167.

[15] A. Grothendieck et J. DieudonNÉ. - Éléments de géométrie algébrique IV. Étude locale des schémas et des morphismes de schémas, Quatrième partie. Publications Mathématiques de l'I.H.E.S, volume 32 (1967), p. 5-361. 
[16] M. Kontsevich et D. Zagier. - Periods. Mathematics unlimited 2001 and beyond, p. 771-808, Springer, Berlin, (2001).

[17] F. Lecomte et N. Wach. - Le complexe motivique de De Rham. Manuscripta Mathematica, Vol. 129, Number 1, p. 75-90 (2009).

[18] M. Levine. - Mixed motives. In, Handbook of $K$-theory, Vol. 1, Friedlander and Grayson, eds., p. 429-521, Springer Verlag (2005).

[19] M. Levine. - Smooth motives. Motives and algebraic cycles, p. 175-231, Fields Inst. Commun., 56, Amer. Math. Soc., Providence, RI, 2009.

[20] C. Mazza, V. Voevodsky et C. Weibel. - Lecture notes on motivic cohomology. Clay Mathematics Monographs, 2. American Mathematical Society, Providence, RI ; Clay Mathematics Institute, Cambridge, MA, 2006. xiv+216 pp.

[21] O. Röndigs And P.-A. Østvar. - Modules over motivic cohomology. Advances in Math., 219(2), p. 689-727, 2008.

[22] J.-P. Serre and J. TATE. - Good reduction of abelian varieties. The Annals of Mathematics, Second Series, Vol. 88, Issue 3, 1968, p. 492-517.

[23] V. Voevodsky. - Cancellation theorem. Documenta Mathematica, Extra Volume : Suslin's Sixtieth Birthday (2010), pp. 671-685.

[24] V. Voevodsky. - Motives over simplicial schemes. Journal of $K$-theory, 5(1), p. 1-38, 2010.

Joseph Ayoub,

Institut für Mathematik, Universität Zürich, Winterthurerstr. 190, CH-8057 Zürich, Switzerland

CNRS, LAGA, Université Paris 13, 99 avenue J.B. Clément, 93430 Villetaneuse, France

- E-mail : joseph.ayoub@math.uzh.ch 